\title{
Electromagnetic Energy Harvesting
}

by

\author{
Melad Olaimat
}

\author{
A thesis \\ presented to the University of Waterloo \\ in fulfillment of the \\ thesis requirement for the degree of \\ Doctor of Philosophy \\ in \\ Electrical and Computer Engineering
}

Waterloo, Ontario, Canada, 2021

(C) Melad Olaimat 2021 


\section{Examining Committee Membership}

The following served on the Examining Committee for this thesis. The decision of the Examining Committee is by majority vote.

External Member: $\quad$ Tayeb A. Denidni

Professor, INRS-EMT, University of Quebec (Universite de Quebec)

Supervisor: $\quad$ Omar Al Ramahi

Professor, Dept. of Electrical and Computer Engineering, University of Waterloo

Internal-External Member: Samer Al Kiswani

Professor, Dept. of Computer Science, University of Waterloo

Internal Member: $\quad$ Rafat Mansour

Professor, Dept. of Electrical and Computer Engineering. University of Waterloo

Zbig Wasilewski

Professor, Dept. of Electrical and Computer Engineering. University of Waterloo 


\section{Author's Declaration}

I hereby declare that I am the sole author of this thesis. This is a true copy of the thesis, including any required final revisions, as accepted by my examiners.

I understand that my thesis may be made electronically available to the public. 


\begin{abstract}
The advent of rectenna systems has opened the door for many applications, with the main goal of harvesting electromagnetic waves. Many designs have been presented to provide a high conversion efficiency. Some works have been focused on the antenna part, where a high-efficient electromagnetic wave to $\mathrm{AC}$ conversion can be achieved. Other works focused on the rectification part, where a high-efficient AC to DC conversion is desired. Even though each part can separately achieve a high conversion efficiency, connecting both parts can dramatically deteriorate the achieved efficiency.

In general, increasing the accuracy of components' characterization leads to a highperformance design. Since the main component in the rectifying circuit is the diode, an accurate model for a nonlinear system is required for efficient use of computer-aided design (CAD) technology. Currently, many models of nonlinear devices consist of basic electric elements such as capacitors, inductors, and resistors. However, building such models needs complex measurement procedures to extract the required parameters precisely. Furthermore, it may take a year to develop and validate an accurate model. Considering challenges associated with traditional approaches of modeling, an accurate and relatively easy method based on X-Parameters to model nonlinear devices has been presented. X-Parameters can be measured at different frequencies and at different power levels and stored in one file. The availability of all information in one file enables us to characterize the systems that operate under unstable conditions, such as the rectenna circuits that are used for energy harvesting.

To accurately simulate a rectenna circuit, we presented a simulation methodology that calls for modeling the antenna part as a circuit that is fully independent in its characteristics from the remaining parts of the rectenna system. This can be accomplished by using Thevenin's theorem. According to Thevenin's theorem, a linear circuit can be replaced with an equivalent circuit consisting of an independent voltage source in series with an impedance. The Thevenin's equivalent circuit provides all the required information about the antenna. It is important to emphasize that the equivalent circuit of the antenna obtained using Thevenin theory is not affected by the rectenna circuit that connects the antenna to the load.

Antennas that can be used both as flat and as curved (conformal antennas) are becoming increasingly attractive antennas because of their wide range of applications. The microstrip patch antenna is one of the most widely used antennas, however, it has a high sensitivity to bending. An antenna with a lower sensitivity to bending is required to avoid the effect of bending on antennas. The metasurface antenna is one of the best candidates to
\end{abstract}


overcome the problem of the antenna's sensitivity to bending. To construct a metasurface, an ensemble of periodic resonators is structured to operate as an effective electromagnetic radiator. In this thesis, we explored the impact of bending on metasurface antennas and microstrip patch antenna arrays. 


\section{Acknowledgement}

First and foremost, I would like to express my sincere gratitude to my supervisor, Professor Omar Ramahi, for his constant support and advice throughout my Ph. D. journey, especially, throughout unprecedented global pandemic. I would like to thank him for his encouragement, motivation, inspiration, patience, passion, and extensive knowledge. During our long and fruitful conversations and discussions, I learned a lot from his knowledge, leadership and experience. Deep inside, I feel privileged to have Professor Omar Ramahi as my $\mathrm{Ph}$. D. supervisor.

I would like to thank my thesis committee: Professor Tayeb Denidni for his valuable time to put in his feedback; Professor Raafat Mansour for the productive discussions that we had in his office and during the useful course that I had with him and for his insightful comments; Professor Samer Al Kiswani for the fruitful discussion we had and for his continuous encouragement; Professor Zbig Wasilewski for his valuable time to discuss this work.

Many thanks go to my fellow lab-mates: Professor Ahmad Ashor, Professor Ali al bishi, Professor Dawood Alsaedi, Professor Leila Yousefi, Professor Maged Aldhaeebi, Professor Mohammad Al badawi, Professor Seyed H. Mirjahanmardi, Professor Sofyan Taya, Professor Tao Tang, Professor Thamer Al moneef, Professor Youcef Braham Chaouche, Professor Vahid Nayyeri, Abdulrahman Aloraynan, Mauricio Hernandez, Shatha Nassef, Saeed Bamatraf, Saleh Ba Raean who where supportive and helpful during this journey.

Last but not least, I would like to thank my family and friends for their support and encouragement. Special thanks to my friend Professor Mohammad Al-sharman and his Wife Dr. Dana Oudat for their support and for the fruitful discussions we had. 


\section{Dedication}

-To my father who did not tell me how to live; he lived and let me watch him do it.

-To my mother, the one person who does the work of twenty. For free.

-To my siblings, who strongly support me every step of the way.

-To my little adorable daughters and son;

Tulene, 4 years old, Kinda, 3 years old, and Adam aged one.

Who where no help in writing this thesis.

-To my beloved wife Dr. Sana'a.

Without your support, this would not have been possible. 


$$
\text { بسم اله الرحمن الرحيم }
$$

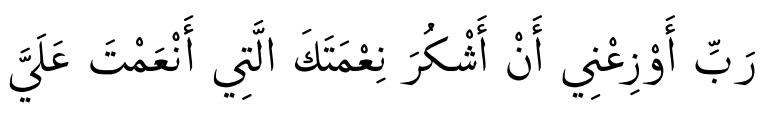

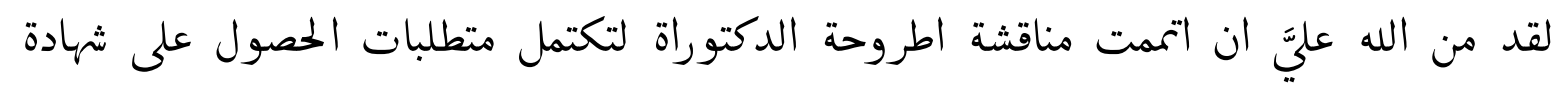

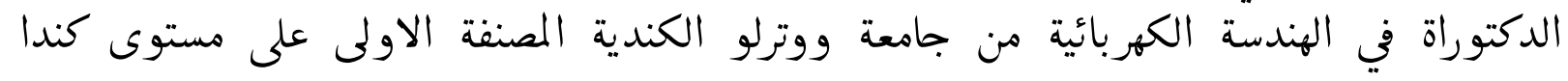

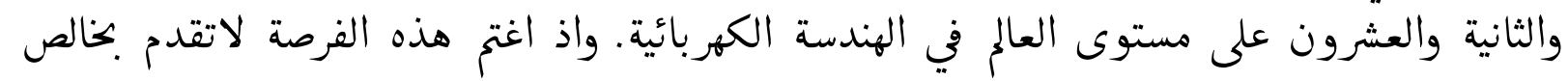

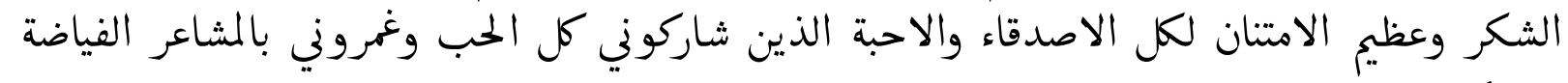

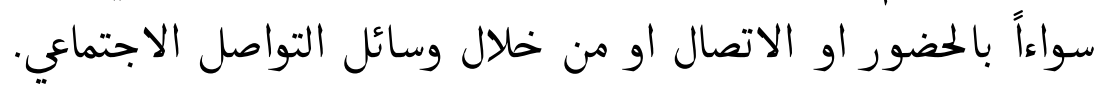

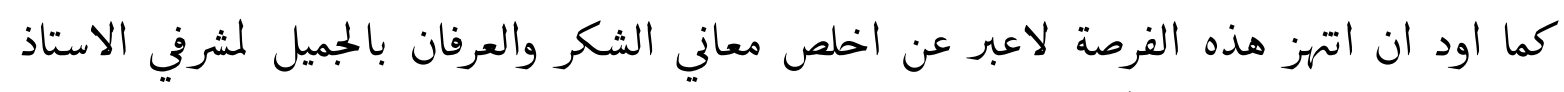

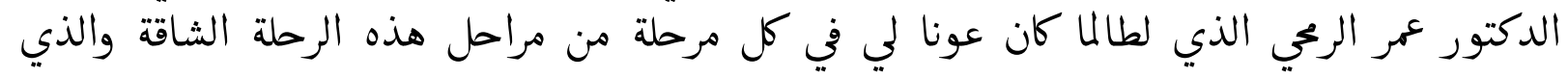

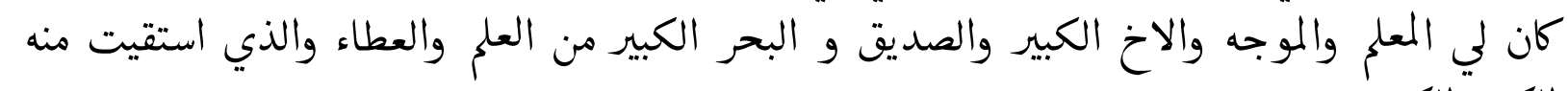
الكثير الكثير.

كما لا يسعني الا ان اقر بالجميل والعرفان لرفيقة دربي وشريكة حياتي الزوجة الغالية الدكتورة

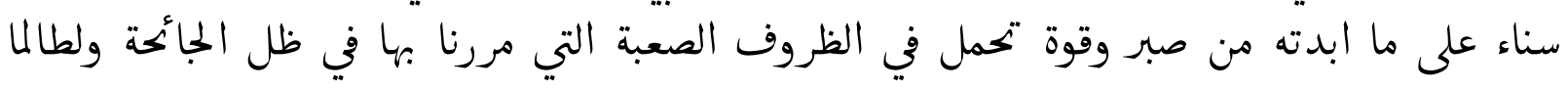

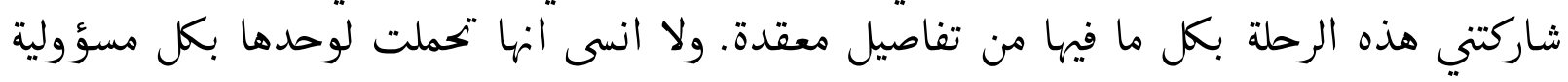

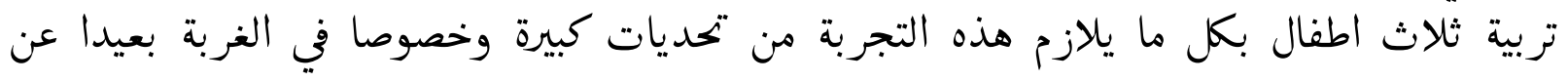

كما اغتم هذه الفرصة لاتقدم من العائلة الكبيرة والدتي العظيمة واخواني واخواتي وانسبائي

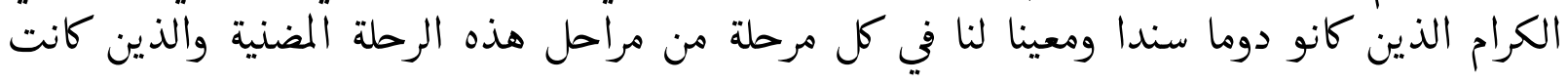

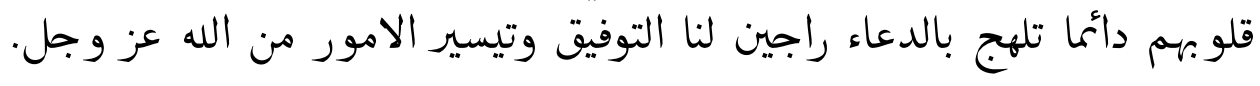

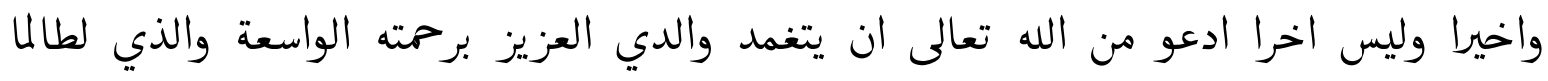

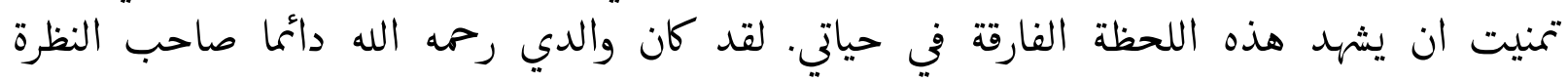

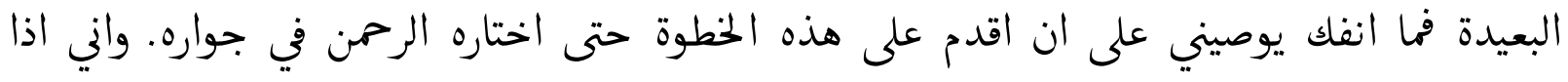
ارجو البارئ عز وجل آن يجعل علمي في ميزان حسناته. 


\section{Table of Contents}

List of Figures $\quad$ xii

List of Tables $\quad$ xviii

1 Introduction $\quad 1$

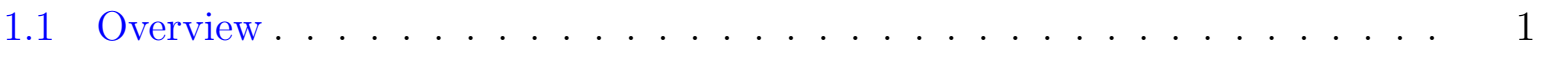

1.2 Energy Harvesting . . . . . . . . . . . . . . . . . . . . 4

1.2.1 Background ...................... 4

1.2 .2 Applications . . . . . . . . . . . . . . . 7

1.3 Literature Review . . . . . . . . . . . . . . . . . . . . 11

1.4 Problem Statement . . . . . . . . . . . . . . . . . . 14

1.4.1 Problem One ........................ . . 14

1.4.2 Problem Two . . . . . . . . . . . . . . . . . . . 15

1.4.3 Problem Three . . . . . . . . . . . . . . . . 16

1.5 Contributions . . . . . . . . . . . . . . . . . . 17

1.6 Thesis Outline . . . . . . . . . . . . . . . . . . 18

2 Rectennas $\quad 20$

2.1 Introduction . . . . . . . . . . . . . . . . . . . . 20

2.2 EM Part . . . . . . . . . . . . . . . . . . . . . . 20

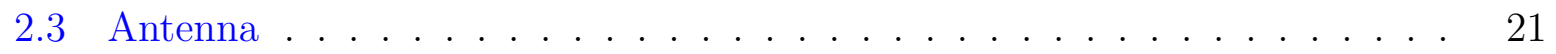


2.3.1 Antenna Parameters . . . . . . . . . . . . . . . . . . . . . . 22

2.3 .2 Antenna Types . . . . . . . . . . . . . . . . . . . . . 24

2.4 Diode . . . . . . . . . . . . . . . . . . . . 26

2.4 .1 Diode Model . . . . . . . . . . . . . . . . . . . . . . . . . 27

2.4.2 Parameters of Schottky Diodes . . . . . . . . . . . . . . 31

2.5 Matching Circuit . . . . . . . . . . . . . . . . 33

2.5.1 Steps of Designing a Matching Circuit Utilizing S-parameters . . . . 33

2.6 Challenges Associated With Designing Rectenna System . . . . . . . . . 39

2.7 Conclusion . . . . . . . . . . . . . . . . . . . . . . . . . . 41

3 X-Parameters in Rectenna's Designs 42

3.1 Introduction . . . . . . . . . . . . . . . . . . . . . . . . 42

3.1.1 Advantages of X-Parameters . . . . . . . . . . . . . . . 44

3.2 Comparison Between X-Parameters and Circuit Model . . . . . . . . . . . 45

3.2.1 Simulation of Diode Using X-Parameters . . . . . . . . . . . 45

3.2.2 Simulation of a Complete Rectenna Utilizing X-Parameters . . . . . 47

3.3 Conclusion . . . . . . . . . . . . . . . . . . . . . . . 57

4 Simulation and Optimization of Rectenna $\quad 58$

4.1 Introduction . . . . . . . . . . . . . . . . . . 58

4.2 Theory . . . . . . . . . . . . . . . . . . . . 60

4.3 Application . . . . . . . . . . . . . . . . . . 65

4.3.1 Design of the Antenna . . . . . . . . . . . . . . 65

4.3.2 Design of Rectifying Circuit . . . . . . . . . . . . 66

4.4 Optimization of Rectenna . . . . . . . . . . . . . . . . . . 72

4.5 Validation . . . . . . . . . . . . . . . . . . . . . . 73

4.6 Conclusion . . . . . . . . . . . . . . . . . . . . . . . . . . . . . . 82 
5 Metasurface Antenna (MSA)

5.1 Introduction . . . . . . . . . . . . . . . . . 83

$5.2 \mathrm{MSA} \ldots \ldots \ldots \ldots \ldots 4 \ldots \ldots \ldots$

5.2 .1 MSA Design . . . . . . . . . . . . . . 84

5.2.2 Feeding Network Design . . . . . . . . . . . . . 85

5.2.3 Results of the Proposed MSA . . . . . . . . . . . . 87

5.3 Effect of Bending on MSA and MPA Array . . . . . . . . . . . . . 89

5.3 .1 Introduction . . . . . . . . . . . . . . . . 89

5.3.2 Comparison Results . . . . . . . . . . . . . 89

5.4 Rectenna System Utilizing MSA . . . . . . . . . . . . . . . . . 93

5.4 Rectenna Design . . . . . . . . . . . . . . . . . . . 94

5.5 MSA Operating in Fifth Generation Band . . . . . . . . . . . . . . . 97

5.6 Conclusion . . . . . . . . . . . . . . . . . . . . . 98

6 Adjustable Input Impedance Dipole For Rectenna System 100

6.1 Introduction . . . . . . . . . . . . . . . . . . . . 100

6.2 Design Methodology . . . . . . . . . . . . . . . . . . 101

6.3 Parametric Study of the Proposed Design . . . . . . . . . . . . . . . . . 102

6.4 Implementation in a Rectenna's Design . . . . . . . . . . . . . . . . . 107

6.5 Conclusion . . . . . . . . . . . . . . . . . . 111

7 Accomplished and Future Work 112

7.1 Accomplished Work . . . . . . . . . . . . . . . . . . . 112

7.2 List of Publications . . . . . . . . . . . . . . . . . . . 114

7.3 Future Work . . . . . . . . . . . . . . . . . . . 115

$\begin{array}{lr}\text { References } & 116\end{array}$ 


\section{List of Figures}

1.1 (a) Share of electric power from renewable sources, (b) Share of electric power from solar, (c) Share of electric power from wind, (d) Share of electric power from hydropower $[1] . \ldots \ldots \ldots 2$

1.2 Solar spectrum analysis of a silicon solar cell [2] . . . . . . . . . . . . . 3

1.3 Potential sources of RF waves [3]. . . . . . . . . . . . . . . . . . 4

1.4 Block diagram of EM energy harvesting categories. . . . . . . . . . . . . . 6

1.5 Concept of Solar Power Satellite System [4] . . . . . . . . . . . . . . . 7

1.6 Architecture of node sensors. . . . . . . . . . . . . . . . . 8

1.7 Domains of applications of WNS. . . . . . . . . . . . . . . . . 9

1.8 Applications of WNS in the healthcare domain. . . . . . . . . . . . . . 10

1.9 Applications of WNS in the environment. . . . . . . . . . . . . . 11

1.10 Circuit diagram of the rectenna system [5] . . . . . . . . . . . . . . . 12

1.11 Responses of a nonlinear device to both small and large input signals. . . . 16

2.1 Rectenna system components. . . . . . . . . . . . . . . . . . . 21

2.2 Equivalent circuit of the EM part. . . . . . . . . . . . . . . . . 22

2.3 (a) General structure of a microstrip patch antenna [6]. (b) Common shapes of microstrip patch antenna $[6] . \ldots \ldots . \ldots 25$

2.4 Classification of natural and artificial materials. . . . . . . . . . . . . . 27

2.5 Current and capacitance variations with the applied voltage in both conventional PN-diode and Schottky diode [7] . . . . . . . . . . . . . 
2.6 Equivalent circuit model of a diode; the components outside the green box are parasitic components. . . . . . . . . . . . . . . . . . 29

2.7 Schematic circuit of a diode with two ports. . . . . . . . . . . 30

2.8 Output harmonics due to $10 \mathrm{dBm}$ input power: (a) Output currents of HSMS2860 at different harmonics. (b) Output voltage of HSMS2860 at different harmonics. . . . . . . . . . . . . . . . . . . . 30

2.9 Schematic circuit of a diode with two ports to extract S-parameters. . . . . 34

2.10 Variation of the reflection coefficient (S11) of the HSMS-2860 diode with frequency and at a fixed input power of $0 \mathrm{dBm} . \ldots \ldots$. . . . . . . .

2.11 Variation of the reflection coefficient (S11) of the HSMS-2860 diode with input power and at a fixed operating frequency of $1 \mathrm{GHz} \ldots \ldots . . . .36$

2.12 Smith chart tool used for designing a matching in ADS with the values of $S_{11} .37$

2.13 Schematic of the obtained matching circuit utilizing smith chart. . . . . . 37

2.14 Schematic circuit of the diode and the matching circuit. . . . . . . . . 38

2.15 Simulated reflection coefficient of the rectifying circuit. . . . . . . . . 38

2.16 Schematic circuit used to simulate the input impedance of the diode. . . . 39

2.17 Variation of diode's input impedance with input voltage for different values of frequencies; (a) Real part (b) Imaginary part. . . . . . . . . . . . . . . . 40

3.1 Difference in response of a nonlinear device to small and large signal. . . . 42

3.2 Two ports network with its input and output. . . . . . . . . . . . . . 43

3.3 X-Parameters extraction simulation layout. . . . . . . . . . . . . . 47

3.4 Comparison between circuit and X-Parameter model layout. . . . . . . . 48

3.5 (a) Simulated voltage obtained from circuit model and X-Parameters model.

(b) Simulated current obtained from circuit model and X-Parameters model. 49

3.6 (a) Top view of the TPA with its dimensions. (b) The fabricated TPA. (c) Coordinate system used showing the angle $\theta$ of the incident field. (d) The fabricated feeding port of the TPA. . . . . . . . . . . . . . 50

3.7 Simulated and measured reflection coefficient, $\mathrm{S}_{11}$, of the proposed TPA. . 51

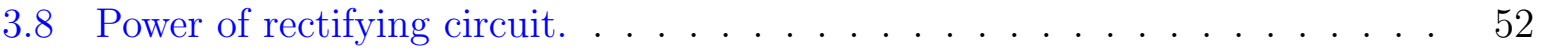


3.9 ADS diode circuit to extract S-parameters. . . . . . . . . . . . . . . 52

3.10 Variation of $S_{11}$ of the diode with frequency at input power of $0 \mathrm{dBm}$. . . 53

3.11 Variation of $S_{11}$ of the diode with input power at frequency of $2 \mathrm{GHz}$. . . 53

3.12 A schematic showing the rectifier circuit designed in ADS. . . . . . . . . 54

3.13 Generation of X parameters of a complete rectenna system. . . . . . . . . . 55

3.14 Comparison between circuit and X-Parameter model layout. . . . . . . . . 55

3.15 The output DC power at $2 \mathrm{GHz}$ in both circuit model and X-Parameters' model. . . . . . . . . . . . . . . . . . . . . 56

4.1 Basic components of a generic rectenna system comprising a receiving antenna, a rectification circuit (may include matching circuits on both ends) and a DC load. . . . . . . . . . . . . . . . . . . . . . 61

4.2 Variation of reflection coefficient of the diode with input power at $2 \mathrm{GHz}$. $\quad 62$

4.3 Equivalent circuit. (a) Thevenin equivalent circuit of an antenna. (b) Representation of the input impedance of the antenna. . . . . . . . . . 64

4.4 (a) Top view of the TPA with its dimensions. (b) The fabricated TPA. (c) Coordinate system used showing the angle $\theta$ of the incident field. (d) The fabricated feeding port of the TPA. . . . . . . . . . . . 66

4.5 Simulated and measured reflection coefficient, $\mathrm{S}_{11}$, of the proposed TPA. . 67

4.6 Schematic diagram of the rectifying circuit with the incorporated equivalent circuit of the antenna. . . . . . . . . . . . . . . . 68

4.7 Simulated $V_{o c}$ for an incident plane wave having power density of $1 \mathrm{~W} / \mathrm{m}^{2}$, polarized in the y-direction and incident at an angle $\theta_{i n}=0 \ldots \ldots$. . . 69

4.8 Simulated impedance of TPA. . . . . . . . . . . . . . . . . . 70

4.9 Simulated power in $\mathrm{mW} \ldots \ldots \ldots \ldots$. . . . . . . . . . . 70

4.10 Power efficiency $\left(\eta_{a c-d c}\right)$ of the rectifying circuit. . . . . . . . . 71

4.11 Simulated output power in $\mathrm{mW}$ optimized at various sub-bands. . . . . . . 73

4.12 Measurements setup; the transmitting side is a horn antenna connected to power amplifier and signal generator and the receiving side is a TPA connected to the rectifying circuit. . . . . . . . . . . . 74

4.13 Laboratory measurement's setup. . . . . . . . . . . . . . . . . . 75 
4.14 Simulated and measured output DC power. The incident field polarization was fixed (y-direction), the incident angle, $\theta$, was fixed at zero degrees (see Fig.4.4), and the power density of the incident field was fixed at $1 \mathrm{~W} / \mathrm{m}^{2}$. .

4.15 Simulated $V_{o c}$ for different power densities of the incident field. The incident field polarization was fixed (y-direction) and incident angle, $\theta$, was fixed at zero degrees (see Fig.4.4). . . . . . . . . . . . . . .

4.16 Simulated $P_{d c}$ for different power densities of the incident field. The incident field polarization was fixed (y-direction) and incident angle, $\theta$, was fixed at zero degrees (see Fig.4.4). . . . . . . . . . . . . . . .

4.17 Simulated $P_{d c}$ for different power densities of the incident field and different frequencies. The incident field polarization was fixed (y-direction) and incident angle, $\theta$, was fixed at zero degrees (see Fig.4.4). . . . . . . . 78

4.18 Simulated $V_{o c}$ values for different angles of incidence. The power density of the incident field was kept constant at $1 \mathrm{~W} / \mathrm{m}^{2} \ldots \ldots \ldots \ldots$. . . . .

4.19 Simulated $P_{d c}$ for different angles of incidence. The power density of the incident field was kept constant at $1 \mathrm{~W} / \mathrm{m}^{2}$. . . . . . . . . . .

4.20 Simulated $P_{d c}$ for different angles of incidence and different frequencies. The power density of the incident field was kept constant at $1 \mathrm{~W} / \mathrm{m}^{2} \ldots \ldots$. .

5.1 Metasurface structure . . . . . . . . . . . . . . . . . . . . . . 85

5.2 (a) The feeding network of the MSA. (b) Quarter-wave transformer. . . . . 86

5.3 Realization of the MSA: (a) ERR (Top) (b) Feeding network (Bottom). . . 87

5.4 (a) Simulated and measured reflection coefficients of the proposed MSA. (b) Simulated and measured gain and efficiency of the proposed MSA. . . . . .

5.5 Simulated and measured normalized radiation patterns of proposed MSA at 5.8 GHz. (a) H-plane. (b) E-plane. . . . . . . . . . . . . . . . . . . 88

5.6 Top view of MPA array. . . . . . . . . . . . . . . . . . . . 90

5.7 (a) Reflection coefficient of MSA and MPA array. (b) Efficiency and gain of MSA and MPA array. . . . . . . . . . . . . . . .

5.8 Structurally deformed MSA with different values of radius: (a) $\mathrm{R}=50 \mathrm{~mm}$, (b) $\mathrm{R}=75 \mathrm{~mm}$, (c) $\mathrm{R}=100 \mathrm{~mm} . \ldots \ldots \ldots \ldots$

5.9 Structurally deformed MPA array with different values of radius: (a) $\mathrm{R}=50$ $\mathrm{mm}$, (b) $\mathrm{R}=75 \mathrm{~mm}$, (c) $\mathrm{R}=100 \mathrm{~mm}$. 
5.10 (a) Reflection coefficient of MSA at different curvatures. (b) Reflection coefficient of MPA array at different curvatures. . . . . . . . . . . .

5.11 (a) Gain of MSA at different curvatures. (b) Gain of MPA array at different

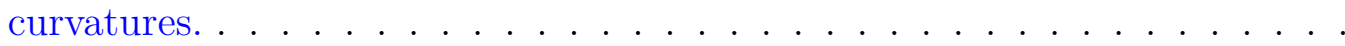

5.12 (a) Efficiency of MSA at different curvatures. (b) Efficiency of MPA array

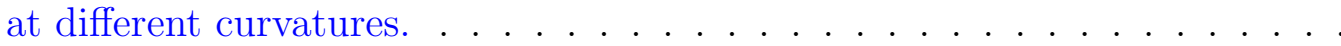

5.13 Basic components of a generic rectenna system comprising a receiving antenna, a rectification circuit (may include matching circuits on both ends) and a DC load. . . . . . . . . . . . . . . . . .

5.14 Schematic diagram of the rectifying circuit with the incorporated equivalent circuit of the MSA. . . . . . . . . . . . . . . 95

5.15 Simulated input and output power of the rectifying circuit in $\mathrm{mW} \ldots \ldots . .96$

5.16 Power efficiency $\left(\eta_{a c-d c}\right)$ of the rectifying circuit. . . . . . . . . 96

5.17 Simulated and measured reflection coefficients of the proposed MSA around $26 \mathrm{GHz} \ldots \ldots \ldots \ldots \ldots \ldots \ldots \ldots$

5.18 (a) Gain of MSA around 26 GHz. (b) Total efficiency of MSA around 26 GHz. 98

6.1 Rectenna system components. . . . . . . . . . . . . . . . . . . . . . . 101

6.2 Schematic showing the antenna design and its parameters. . . . . . . . 102

6.3 Simulated input impedance of the proposed antenna. The input impedance is $187+\mathrm{j} 39 \Omega$ at $3 \mathrm{GHz} \ldots \ldots \ldots \ldots \ldots \ldots$

6.4 Simulated reflection coefficient using port of $187+\mathrm{j} 39 \Omega$ reference impedance. 104

6.5 Real part of the simulated input impedance of the proposed design for different $L_{2} \ldots \ldots \ldots \ldots \ldots \ldots \ldots \ldots$

6.6 Imaginary part of the simulated input impedance of the proposed design for different $L_{2} \ldots \ldots \ldots \ldots \ldots \ldots \ldots$

6.7 A schematic showing the rectifier circuit designed in the ADS simulator. The transmission lines parameters are listed in Table 6.5. The circuit is terminated by a parallel combination of load resistor of a $4.5 \mathrm{~K} \Omega$ and a 40 pF capacitor. . . . . . . . . . . . . . . . . . . . 108

6.8 Simulated input impedance of the proposed antenna. The input impedance is $159.5+\mathrm{j} 12 \Omega$ at $2 \mathrm{GHz} \ldots \ldots \ldots \ldots \ldots$ 
6.9 A schematic showing the rectifier circuit designed in ADS. The parameters are listed in Table 6.6. There is a total of 6 microstrip transmission line segments where TLn refers to the nth segment. The circuit is terminated by a parallel combination of load resistor of $4.5 \mathrm{~K} \Omega$ and a capacitor of $40 \mathrm{pF} .110$

6.10 Conversion efficiencies; using adjustable antenna without a matching circuit, using $50 \Omega$ antenna without a matching circuit and using $50 \Omega$ antenna with a matching circuit. . . . . . . . . . . . . . . . . 110 


\section{List of Tables}

2.1 Advantages and disadvantages of MA. . . . . . . . . . . . . . 25

2.2 Parameters of Schotkky diode HSMS-28xx series [8] . . . . . . . . . . . 33

3.1 Parameters of the transmission lines segments of the matching circuit . . . 54

3.2 Output voltage of the rectenna system using both circuit model and $\mathrm{X}$ Parameters. . . . . . . . . . . . . . . . . . 57

4.1 Lengths of the transmission lines segments used in the optimized rectification circuit. . . . . . . . . . . . . . . . . . . . . 69

4.2 Parameters of the rectification circuits optimized at various bands (R). . . 72

6.1 Simulated input impedance of the proposed design for different $L_{2}$. Other antenna parameters were fixed at $L_{1}=23.3 \mathrm{~mm}, W_{1}=W_{2}=2 \mathrm{~mm}$ and $\mathrm{g}=1 \mathrm{~mm} .104$

6.2 Simulated input impedance of the proposed design for different $W_{2}$. Other antenna parameters were fixed at $L_{1}=23.3 \mathrm{~mm}, L_{2}=6 \mathrm{~mm}, W_{1}=2 \mathrm{~mm}$ and $\mathrm{g}=1 \mathrm{~mm} \ldots \ldots \ldots \ldots \ldots \ldots \ldots . \ldots \ldots \ldots$

6.3 Simulated input impedance of the proposed design for different $W_{1}$. Other antenna parameters were fixed at $L_{1}=23.3 \mathrm{~mm}, L_{2}=6 \mathrm{~mm}, W_{2}=2 \mathrm{~mm}$ and

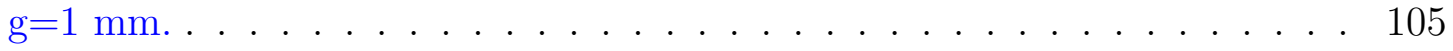

6.4 Simulated input impedance of the proposed design for various gaps (g). Other dimensions were fixed at $L_{1}=23.3 \mathrm{~mm}, L_{2}=6 \mathrm{~mm}$ and $W_{2}=W_{1}=2 \mathrm{~mm} .105$

6.5 Parameters of the transmission lines segments of the matching circuit in Fig.

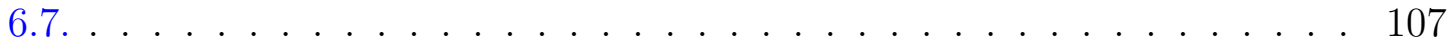

6.6 Parameters of the transmission lines segments of the rectenna system in Fig. $6.9 \ldots \ldots \ldots \ldots \ldots \ldots \ldots \ldots$ 


\section{Chapter 1}

\section{Introduction}

\section{$1.1 \quad$ Overview}

Access to energy is a key pillar for economic development and poverty alleviation. Currently, global energy demand is greatly increasing, due to the increase in population and economic development. The increasing energy demand and the rapid decrease in fossil fuel reserves lead to an urgent need for attainable renewable energy resources. Driven by unprecedented demands on energy, the need for new paradigms that produce clean and affordable energy becomes increasingly desired. Energy Harvesting (EH) is one of the promising and satisfactory techniques that may contribute in some way to solving the energy shortage problem. $\mathrm{EH}$ is the technique that is utilized to scavenge or harvest the available ambient energy in nature. The fundamental advantage of this sort of energy over others is that it is abundantly available in the environment and does not require any effort to generate.

Figure 1.1a shows the share of electric power from renewable sources during the last two decades [1]. As shown in the figure, of the total world's electricity power generated, the share of the electric power from renewable sources increased from $19 \%$ to $29 \%$ between the periods from 2000 to 2019. Figure 1.1b depicts the growth of the share of the electric power from solar sources. It can be noted that there has been an exponential growth in the utilization of solar sources to generate electrical power since 2000, and the share increased from almost zero to more than 3.4\%. The growth of the share of the electric power from wind energy has increased exponentially as well, and it has been increasing since the 2000s (from $0.1 \%$ to more than 6.5\%) as illustrated in Fig. 1.1c. Observing Figure 1.1d, the share of electricity from hydro-power is larger than $16 \%$ during the last two decades [1]. 


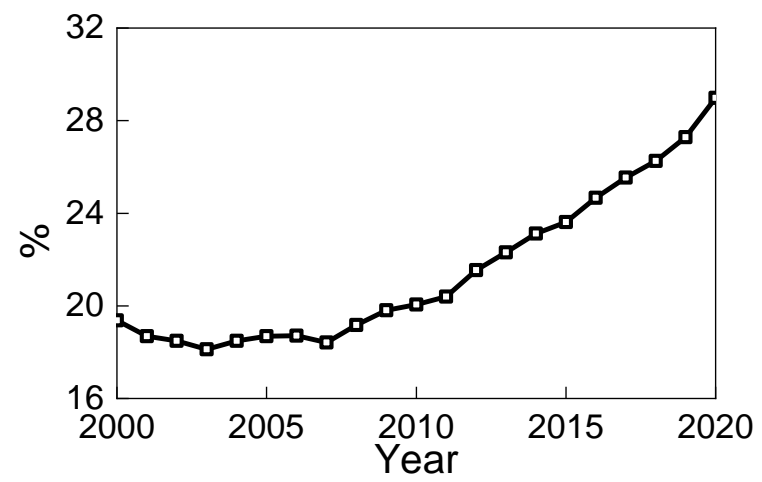

(a)

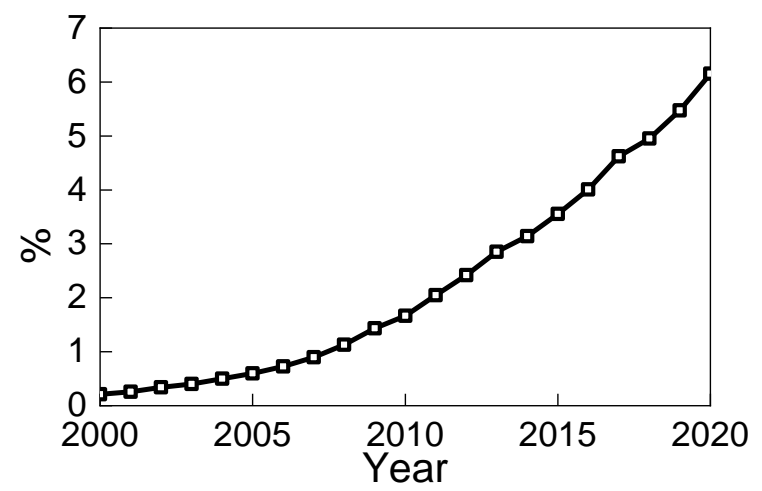

(c)

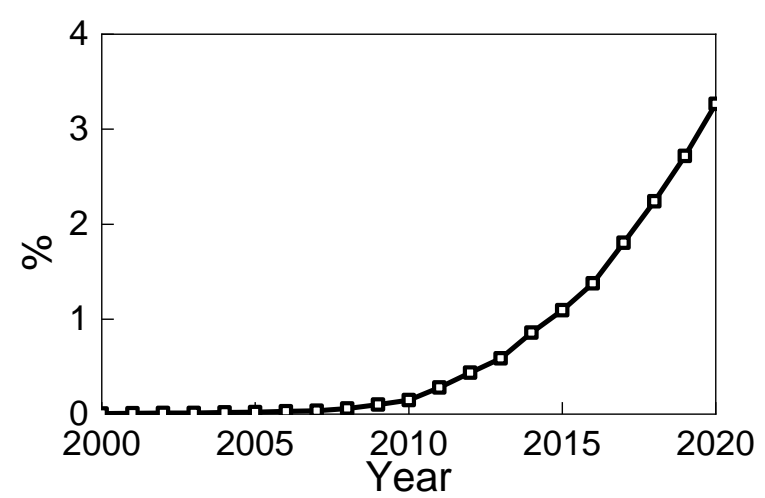

(b)

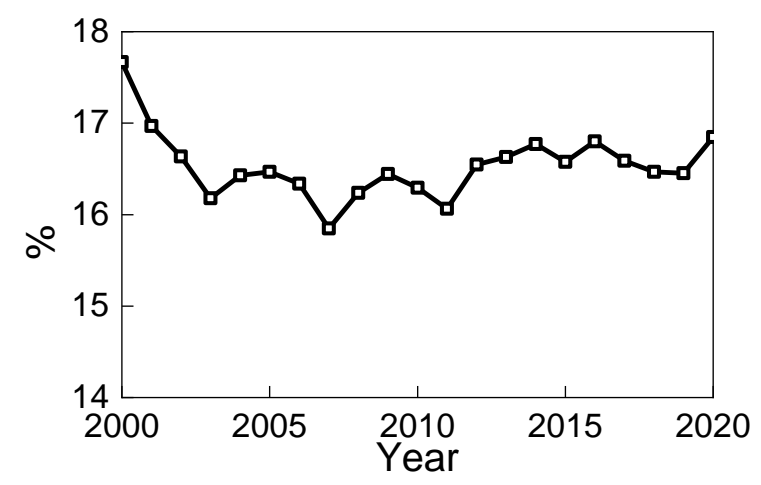

(d)

Figure 1.1: (a) Share of electric power from renewable sources, (b) Share of electric power from solar, (c) Share of electric power from wind, (d) Share of electric power from hydropower [1].

Although intensive research has been conducted to come up with creative technologies that are capable of exploiting energy from renewable sources,numerous challenges limit the efficiency of harvesting the energy from these sources. For instance, the sun is a valuable source of energy, and the daily amount of energy that reaches the atmosphere from the sun is equal to the human energy needs for a year. The atmosphere, approximately, reflects one-third of this energy, additionally, it absorbs about $19 \%$ of the coming energy and reradiates it to the surface of the earth as infrared (IR) waves at a wavelength ranging from 7 to $14 \mu \mathrm{m}$. The surface of the earth absorbs the remaining energy and reradiates it at a wavelength of around $10 \mu \mathrm{m}(30 \mathrm{THz})$.

Photo-voltaic (PV) cells are nowadays the most widely used devices for converting 
solar energy into electrical energy. PV cell technologies, on the other hand, have several drawbacks, including low conversion efficiency due to semiconductor band-gap and heat dissipation, which limit the maximum theoretical efficiency of a single-junction solar cell to only 31\%; and high cost due to material and processing costs involved in the production [9]. Silicon solar cells, for example, have captured a significant share of the total PV market, owing this to their high efficiency [10]. The conversion efficiency of the conventional thick wafer reaches as high as $26.3 \%$ for single junction and $31.3 \%$ for multi-junction solar cells $[11,12]$. This low efficiency is because of the thermalization of photo-generated carriers in the crystal lattice [13], thermodynamic losses, and unabsorbed photons [14]. Figure 1.2 shows the spectral analysis of the minimum losses for a silicon solar cell (bandgap $=1.1$ $\mathrm{eV}$ ). As illustrated in the Figure, the available free energy from an ideal present-day singlejunction cell is roughly $33 \%$, another $33 \%$ is lost due to thermalization and the remaining third is split between photons not absorbed and unavoidable thermodynamic losses [14].

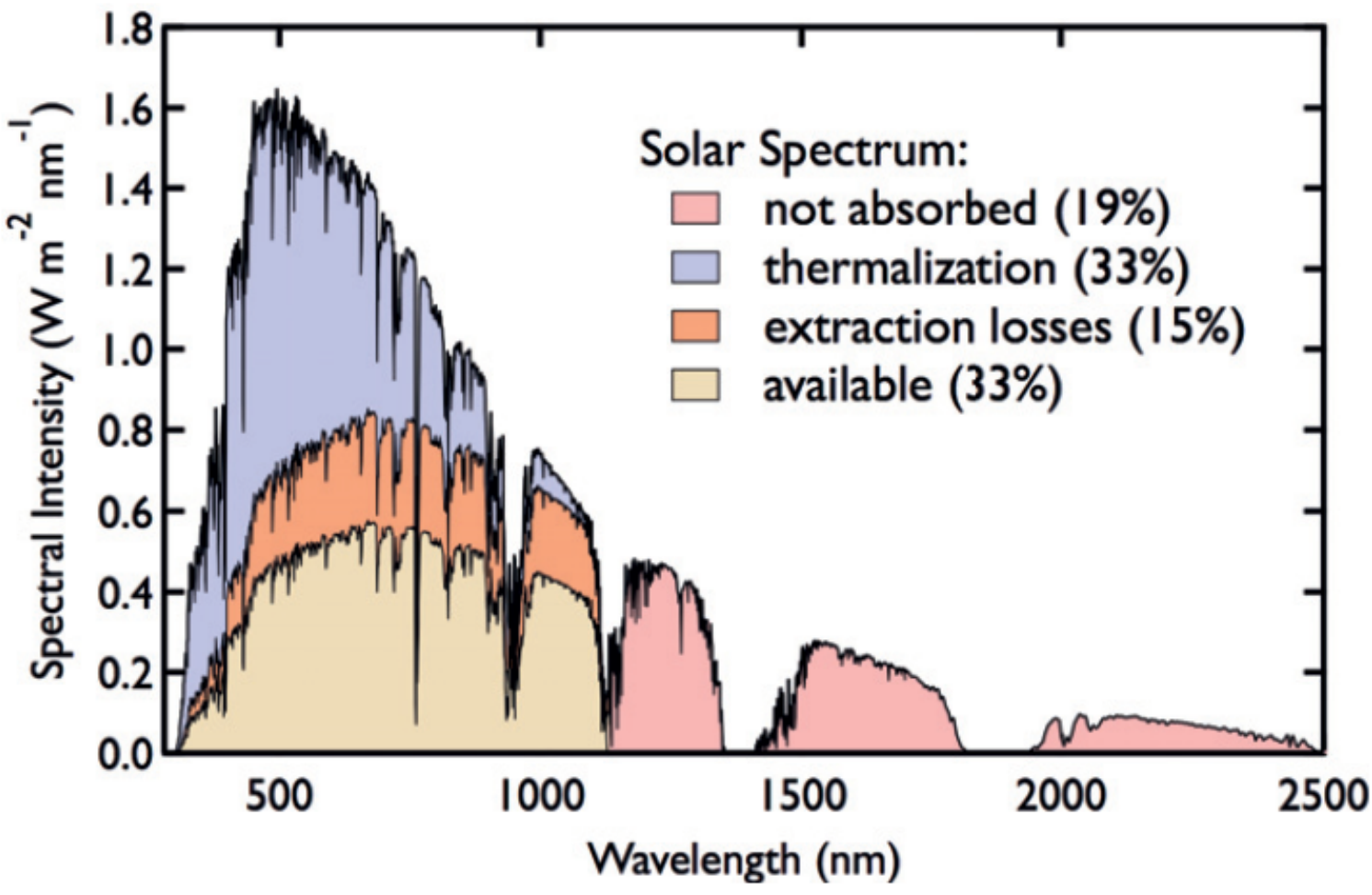

Figure 1.2: Solar spectrum analysis of a silicon solar cell [2].

Inspired by the availability of electromagnetic (EM) energy, the idea of EM energy harvesting was originally proposed by Bailey [15]. Figure 1.3 shows potential sources of 
electromagnetic waves. Following Bailey's findings, a slew of research has been conducted to see if a system called rectenna, which comprises antennas and rectification circuits, can be used to harvest EM energy. Many works have been published to overcome the challenges that are related to harvesting EM energy utilizing rectennas [16, 17]. To achieve high-efficiency free space to AC conversion, some study has focused on the antenna portion [17]. Other research focuses on the rectification process, which requires a high-efficiency $\mathrm{AC}$ to DC conversion. Even though each part can separately achieve a high conversion efficiency, connecting both parts can dramatically deteriorate the achieved efficiency.

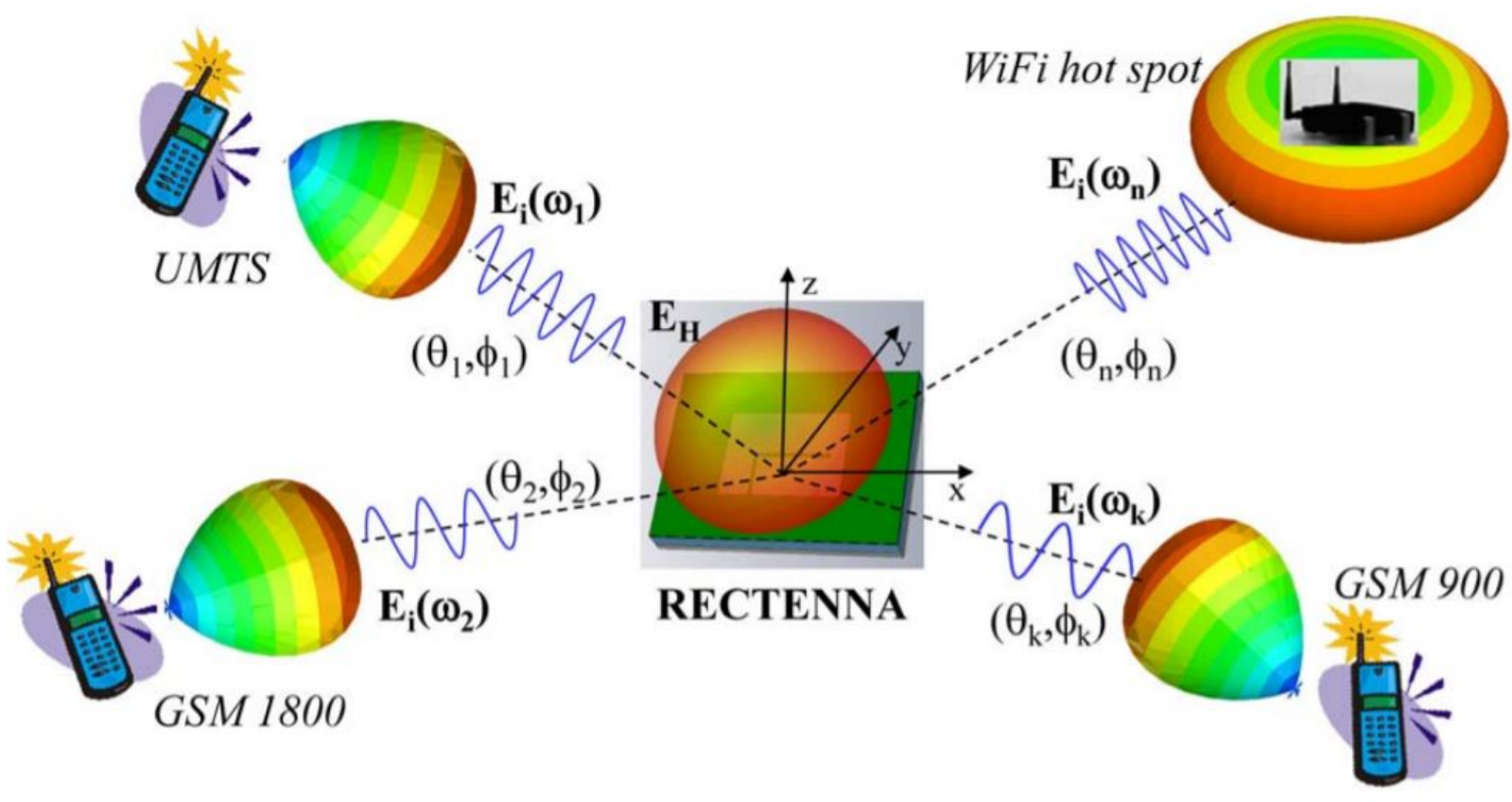

Figure 1.3: Potential sources of RF waves [3].

\section{$1.2 \quad$ Energy Harvesting}

\subsubsection{Background}

Generally, EM energy harvesting can be classified into two categories; wireless power transfer (WPT) and ambient EM wave harvesting as illustrated in Fig.1.4. WPT can be achieved 
over a short distance (near field) or a long-distance (far-field). There is no sharp border between near field and far field, however, the near field can be considered as a field confined within a region of one wavelength radius from a radiator. In this region, the power can be transferred by electric field utilizing capacitive coupling, or by magnetic field utilizing magnetic coupling. In the near field, there is no propagation and the power does not leave the transmitter unless there is a receiving device in the near field region. Indeed, the power transmission utilizing near field is limited in range, because the field decreases exponentially with distance. Originally, transferring the power without cables utilizing coupling between two coils was experimentally demonstrated by Michael Faraday in 1831. The coupling between coils drastically decreases as the spacing between them increases, which limits the efficiency of transferring the power over a relatively long distance [18].

In 1865, Maxwell mathematically demonstrated that electric and magnetic fields can propagate in space as EM waves at the speed of light. The formulated mathematical model predicted the existence of EM waves [19]. However, due to the lack of equipment and technology at that time, he couldn't experimentally prove the existence of EM waves. After a while, Hertz could experimentally confirm the existence of EM waves predicted by Maxwell's equation. Hertz utilized a half-wavelength dipole to send and receive power at a frequency of $500 \mathrm{MHz}$ for the first time leading to the birth of far-field WPT. In 1898, Tesla generated power at $150 \mathrm{kHz}$ and lighted 200 light bulbs 26 miles away from the transmitter [20]. Due to the lack of high power sources that operate at high frequency at that time, Tesla's projects on WPT stopped in 1910.

The development of high-power microwave tubes in the 1950s provided a solution to the problem of generating high-power high-frequency signals which increased the interest in studying the feasibility of the WPT concept. A source of a continuous wave of $400 \mathrm{KW}$ that operates at $3 \mathrm{GHz}$ was presented by Raytheon company in 1960 with an efficiency of $80 \%$ [21]. In the same year, the same company developed a rectenna system that consists of a $2.45 \mathrm{GHz}$ half-wave antenna connected with a rectifying circuit, and the maximum conversion efficiency of 50\%. 4 years later, motivated by the success of Raytheon company in demonstrating the feasibility of WPT, William Brown successfully built a far-field WPT system to provide a helicopter with the required power remotely as an application of WPT.

In 1968, Space Solar Power (SSP) was introduced by Glasser [22]. Figure 1.5 shows the concept of SSP. In this system, huge concentrating mirrors are utilized to concentrate the light of the sun at solar cells. The absorbed solar power is converted into microwave signals that are transmitted to the earth by highly directive antennas. The received power on the earth is harvested by a farm of rectenna array systems that convert the RF power into useful DC power. In near future, it is expected to obtain a conversion efficiency of $80 \%$ from DC to microwave. The loss of the RF signal below $6 \mathrm{GHz}$ due to the atmosphere is less than $3 \%$ on 


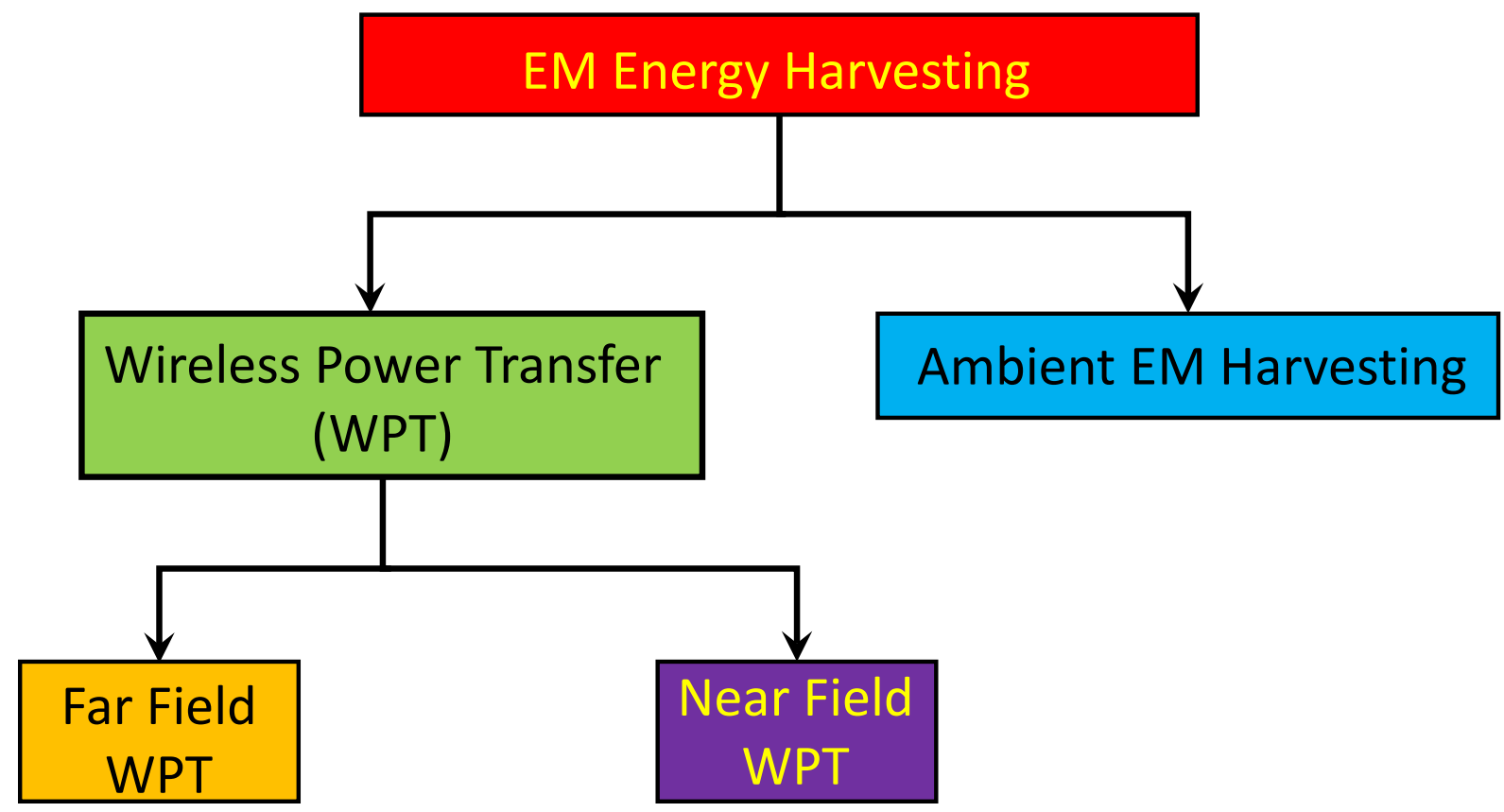

Figure 1.4: Block diagram of EM energy harvesting categories.

sunny days, and it is expected to be around $8 \%$ on moderately rainy days. Considering the losses due to the conversion from DC to RF and vice versa and the propagation attenuation, the total loss due to wireless power transmission/reception is estimated to be around $50 \%$. Despite the loss caused by the additional transmission/reception process, the SSP is still competitive with ground solar cells due to its continuity during day and night time [23].

The introduction of the SSP concept increased the interest in the WPT system and many projects have been launched to investigate the feasibility of the SSP concept. For example, NASA supported two research groups to investigate the feasibility of SSP. In 1975, one of the research groups (Jet Propulsion Laboratory (JPL)) demonstrated experimentally the feasibility of receiving $30 \mathrm{KW}$ DC power at a distance of 1 mile from the transmitter. In this experiment, they utilized a parabolic antenna operating at $2.4 \mathrm{GHz}$ as a transmitter and an array of dipole rectennas at the receiving side. To wirelessly power an aircraft, Canada launched the stationary high-altitude relay program in 1980. A small-scale airplane was flown at $50 \mathrm{~m}$ height for 20 minutes utilizing a $10 \mathrm{KW}$ ground station. The power was transmitted by a parabolic dish antenna with an operating frequency of $2.45 \mathrm{GHz}$. To convert the received RF power into DC power, the airplane was equipped with an array of rectennas attached to the lower surface of the airplane's wings [24]. To investigate the 


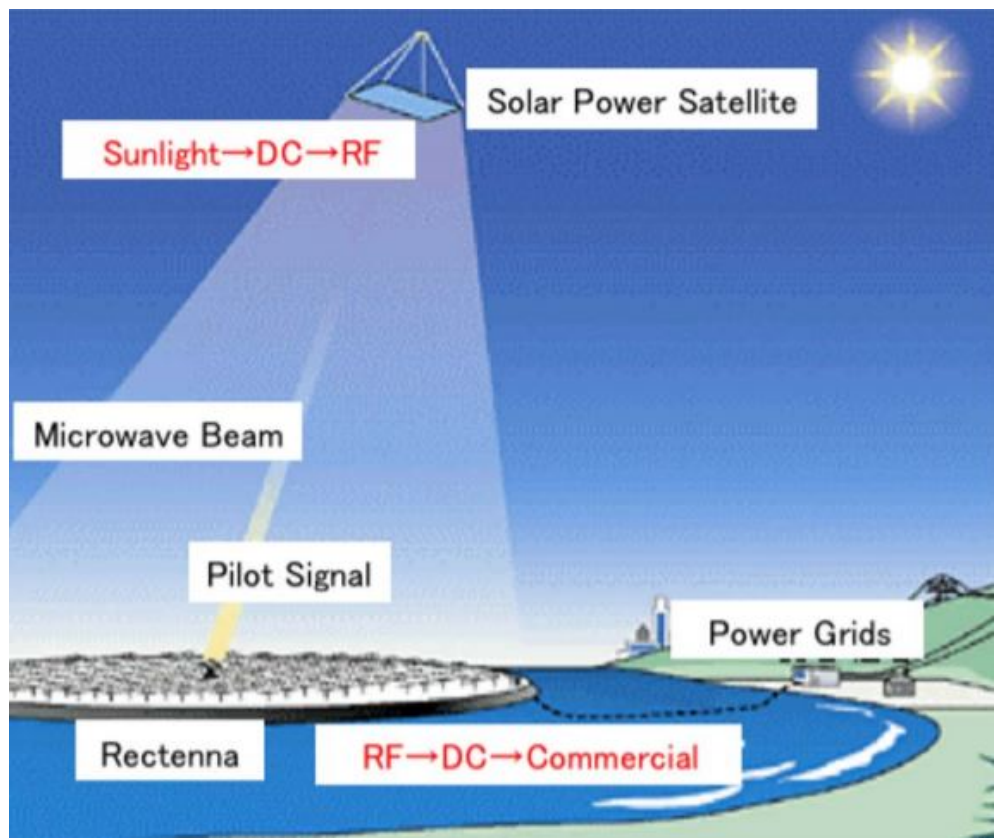

Figure 1.5: Concept of Solar Power Satellite System [4].

effect of transferring power through the Ionosphere, Japan launched its project (Microwave Ionosphere Nonlinear Interaction Experiment (MINIX)) in 1984 [25]. In the 1990s, as a fruit of collaboration between Texas A\&M University and Japan, an experimental demonstration showed the ability to send power between two rockets. In this project, power of $800 \mathrm{~W}$ was transmitted from one rocket at $2.45 \mathrm{GHz}$. The transmitted power was received at the second rocket utilizing dual-polarized rectenna [26].

\subsubsection{Applications}

Currently, WPT is utilized in many applications including, but not limited to, wireless charging biomedical implantable devices, charging the batteries of Wireless Network Sensors (WNS), Radio Frequency Identification (RFID), and charging mobile equipment $[27,28,29,30]$. Although the wide range of applications of WPT, SSP became the most attractive application after the announcement of the Japanese plans to launch a commercial SSP system targeting 1GW power in 2030 [23]. The diverse potential applications of energy harvesting and rectenna systems necessitate accurate design and assessment strategies of rectenna systems. 
The availability of internet wireless access facilitates and accelerates the application of the Internet of Things (IoT) concept. In this concept, a network of physical objects that are occupied with sensors and software are connected and to the internet to facilitate the sharing of data. Wireless Sensor Networks (WSNs) are essential components in the IoT concept to monitor and send data from a wide range of areas. A WSN can be described as a group of wirelessly interconnected nodes that are distributed over a specific area [31].

Generally, each sensor node consists of a sensing unit, processing unit, communication unit, and a power unit as depicted in Figure 1.6. The sensing unit measures the fluctuation of the required quantity utilizing its sensors and translates the variations into a useful electrical signal. The electrical signals are processed and stored by the processing unit. The communication unit is responsible for connecting the node wirelessly with other nodes by transmitting and receiving the data from and to the node. As shown in the Figure all units need a power unit to provide them with a proper amount of power [32]. To prolong the lifetime of WSN, their battery should be periodically charged because of their limited lifetime. Due to their spread over a wide geographical area, charging the batteries of WSN is challenging and impractical unless they are provided with an efficient wireless charging system. Rectenna system provides a viable option to wirelessly charge the batteries of WSN from the ambient EM wave.

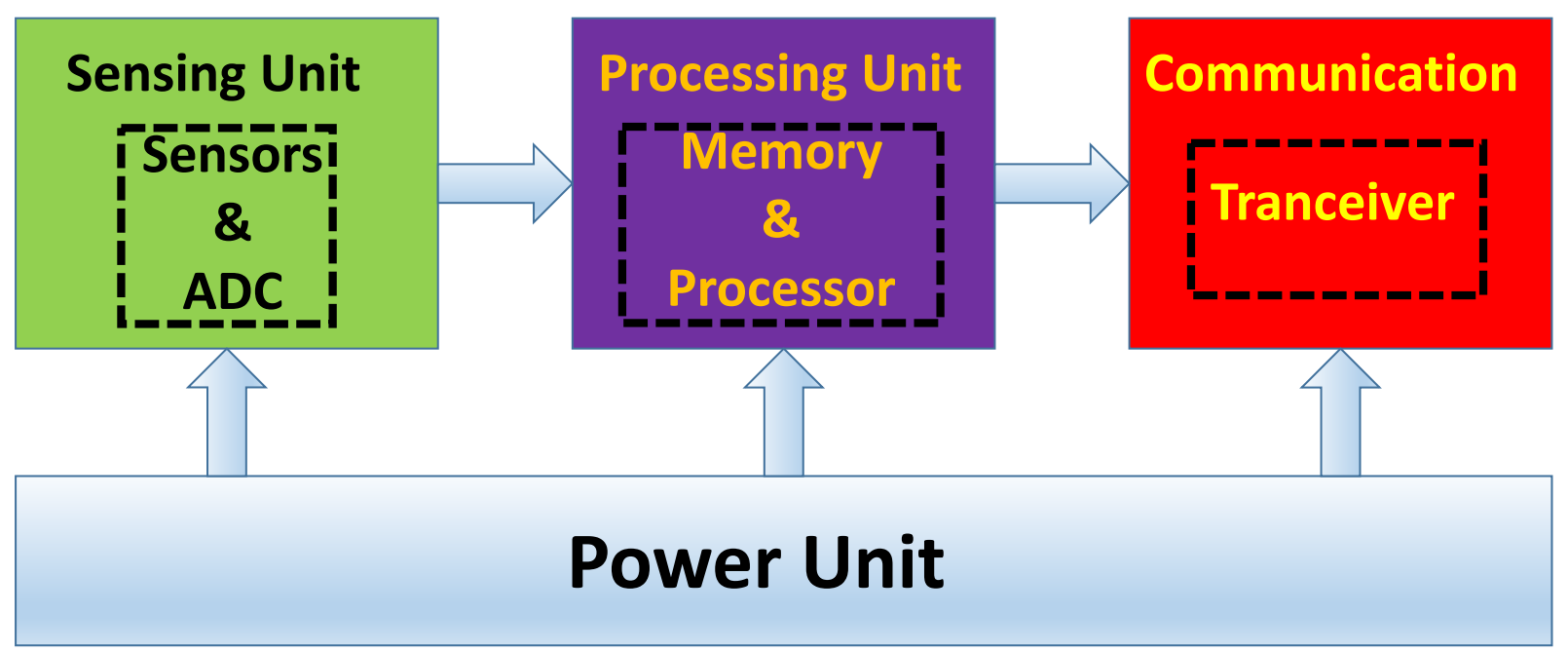

Figure 1.6: Architecture of node sensors.

Recently, WSNs have become an attractive field of research due to their wide range of applications. Low prices and small size make them ideal for a wide range of applications. 
Given this wide range of applications, it is not surprising that their applications penetrate all aspects of our life. According to the nature of their usage, the applications of WSN can be classified into six main categories; health, environmental, flora and fauna, industrial, urban and military, as illustrated in Figure 1.7 [32]. Each category includes many applications of WSN, some of them are mature and others are still in the development stage.

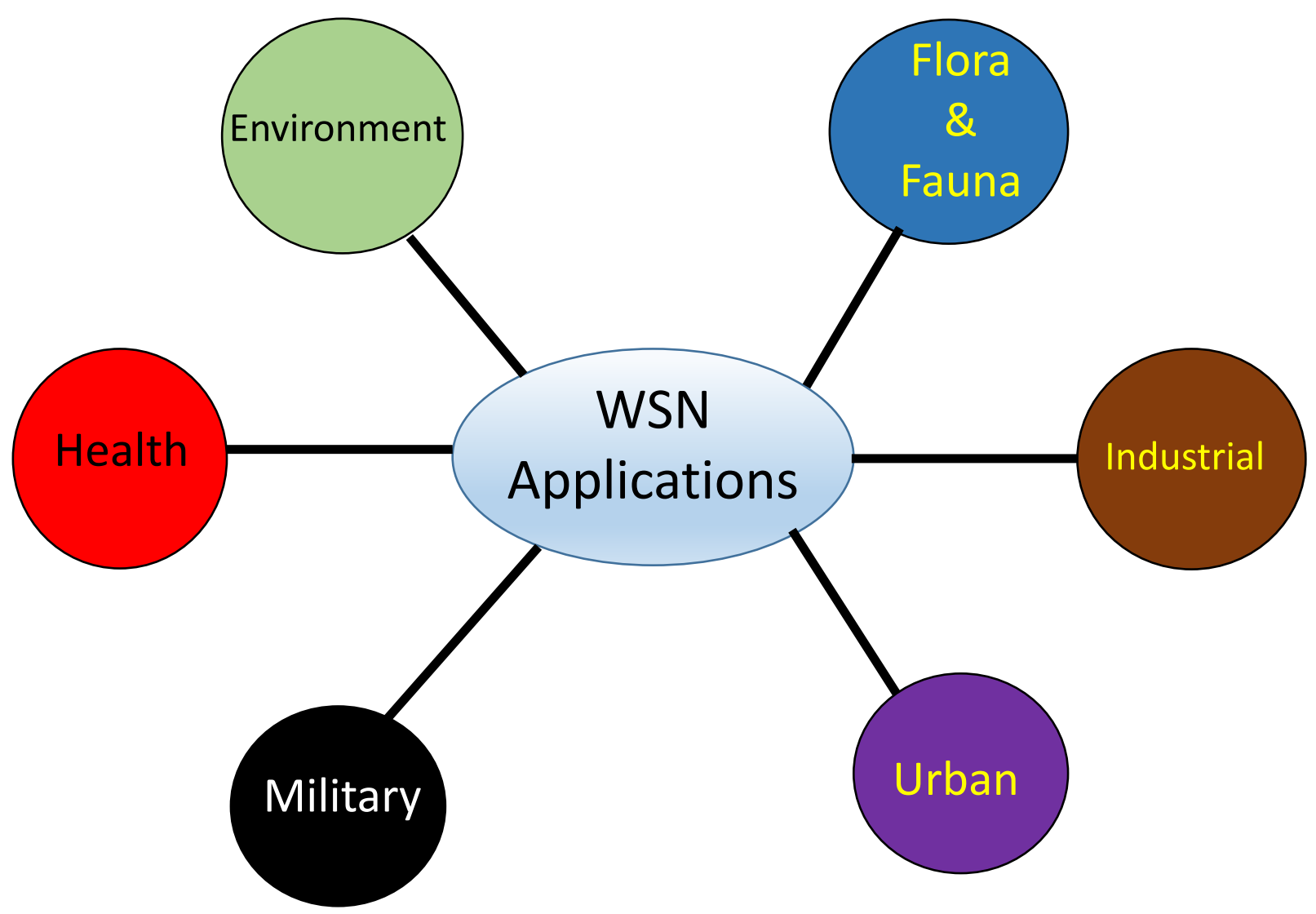

Figure 1.7: Domains of applications of WNS.

In healthcare, the application of WSNs can improve the operation of a healthcare facility via monitoring real-time vital functions of the patients utilizing advanced medical sensors. The WSNs can be employed in patient wearable monitoring, home assisting systems and hospitalization. Figure 1.8 shows some potential applications of WNS in the healthcare domain along with the type of sensors required for each application. For instance, implanted biomedical sensors can be combined with health monitoring applications 
to provide real-time status of blood pressure and blood glucose levels, and different types of diagnostics [32, 33]. For home assisting purposes, the patient can be remotely connected to the internet via WSN to send real-time or periodic readings about his status or send an alarm in case of an emergency. In hospitals, monitors attached to or implanted in patients can be connected directly to the doctors and nurses via a local network [34, 35].

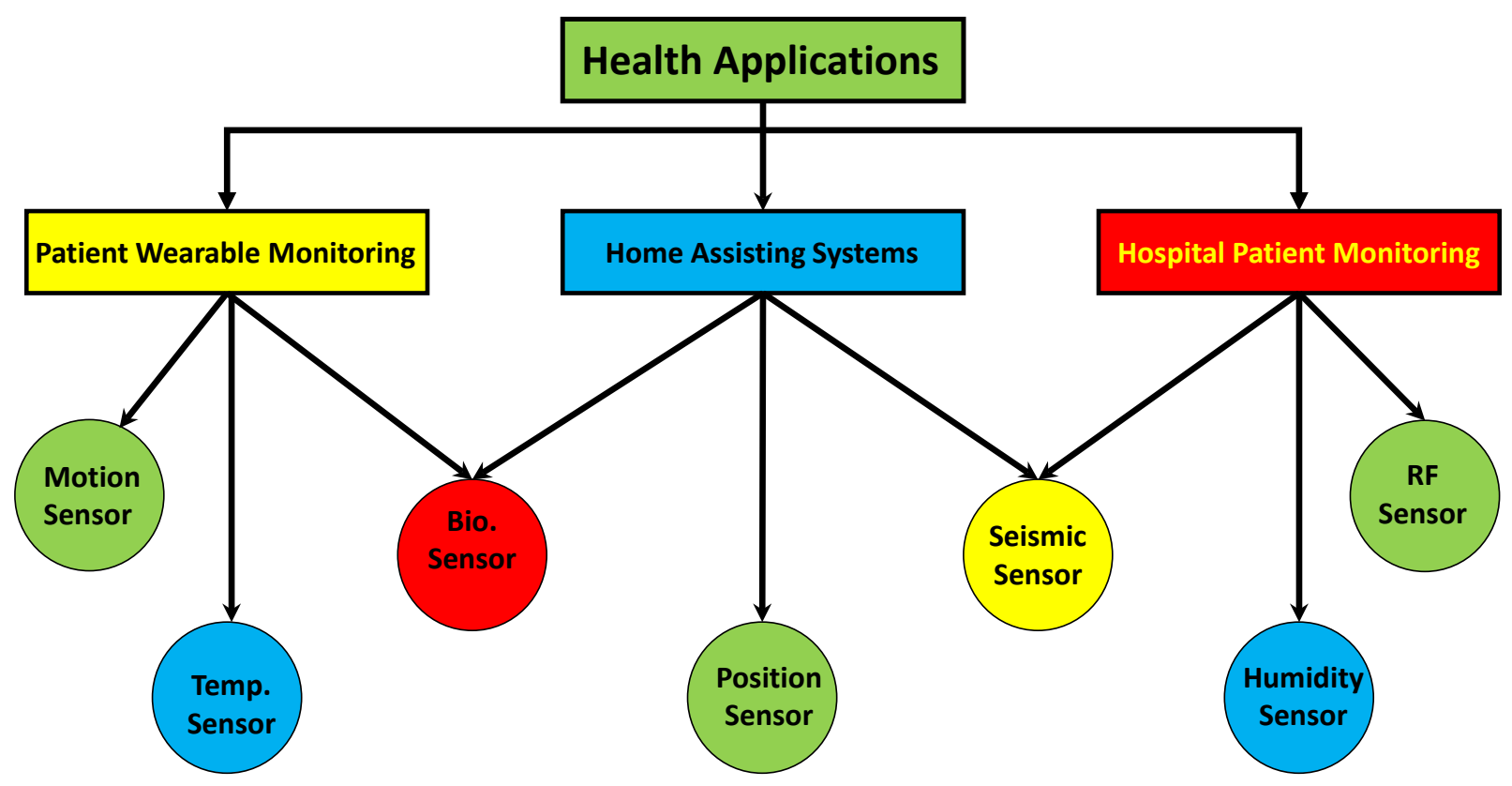

Figure 1.8: Applications of WNS in the healthcare domain.

WSN can help in monitoring many environmental parameters including, but not limited to, water quality, air pollution, and humidity. Furthermore, it enables us to early detect forest fires, landslides and natural disasters. The availability of enough data about environmental parameters and early detection of crises enables us to efficiently deal with environmental phenomena and help in avoiding the bad consequences. Figure 1.9 shows some applications of WSN in environmental monitoring. As depicted in the Figure, the applications can be categorized into three categories; water monitoring, air monitoring, and emergency alerting. Each category requires special types of sensors that fit their nature.

Water, either drinkable or ocean water, is one of the most important environmental entities on the earth, and monitoring its quality comes at the top priority of environmental scientists. The WSNs are intensively employed in monitoring the water quality and water distribution systems. For instance, the researcher in [36] utilized a Radio Frequency Identification (RFID) based WSN system to monitor the quality of water in the pipeline, 
moreover, the system can be utilized to report any problem with the pipeline itself. To avoid the damage of fauna and flora due to the fish farm waste, an underwater WSN that can detect the pollution was presented in [37]. The modern human lifestyle and activities considerably harm the atmosphere and cause severe air pollution. To monitor the pollution and the concentration of each element in the air, the WSN can be utilized. For example, to measure the concentration of gases such as CO and NO2 gas sensors were utilized [32].

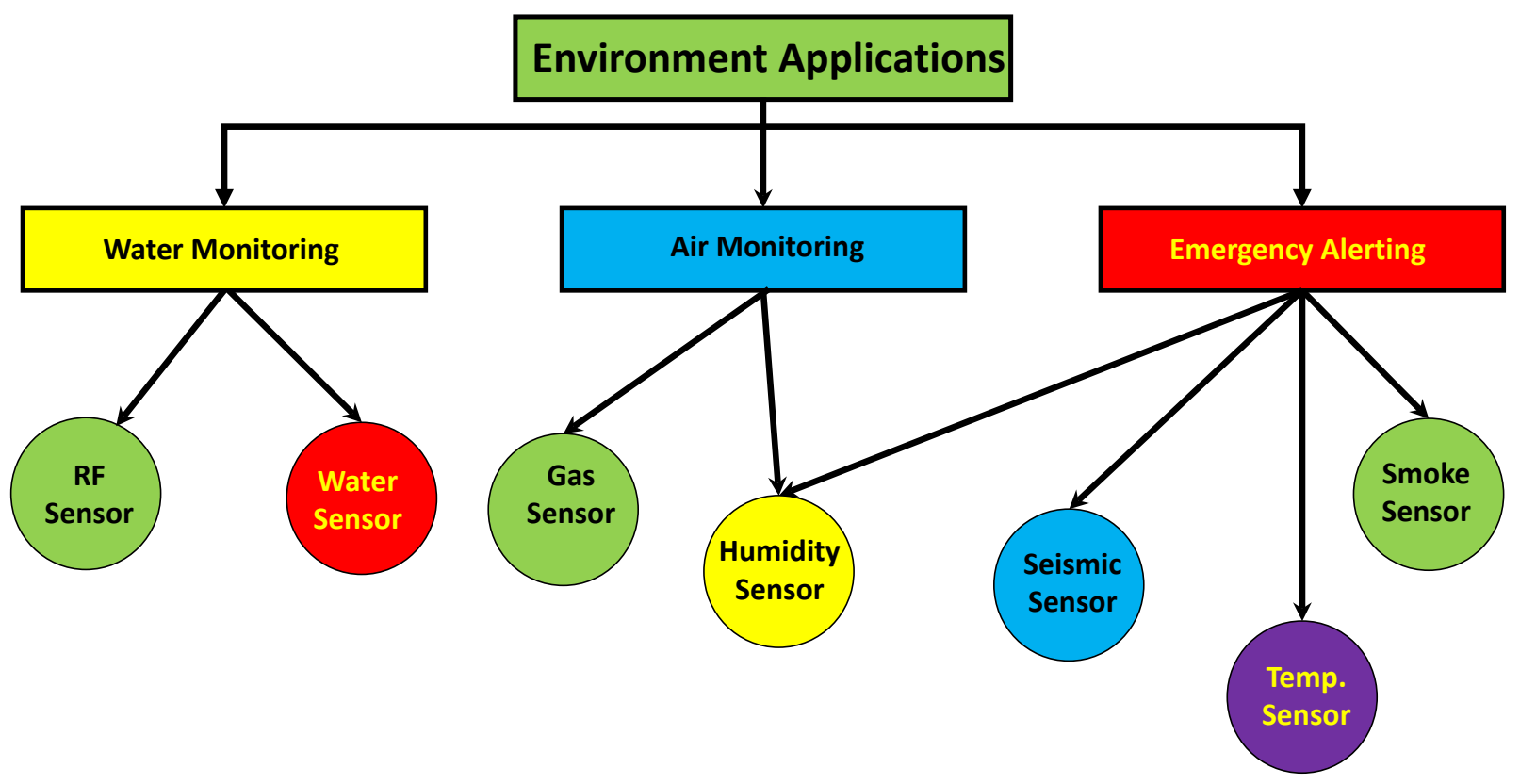

Figure 1.9: Applications of WNS in the environment.

\subsection{Literature Review}

Recently, there has been intensive research to investigate the ability of rectenna systems to harvest the energy from the electromagnetic (EM) radiation (for a sample of recent works, please see $[38,39,40,41,42,43,44,45,46,47,48,49,50,51,52]$ and references therein). To harvest EM radiation, a rectenna is used, which consists of an antenna or a surface that captures the EM radiation, a rectifying circuit, and a DC load circuit as illustrated in Fig. 1.10. The antenna acts as a transducer that converts the incoming EM radiation into an electrical signal. The rectifying circuit, which consists of a single or multiple diodes and a matching circuit, converts the AC power into DC power. 


\section{antenna}

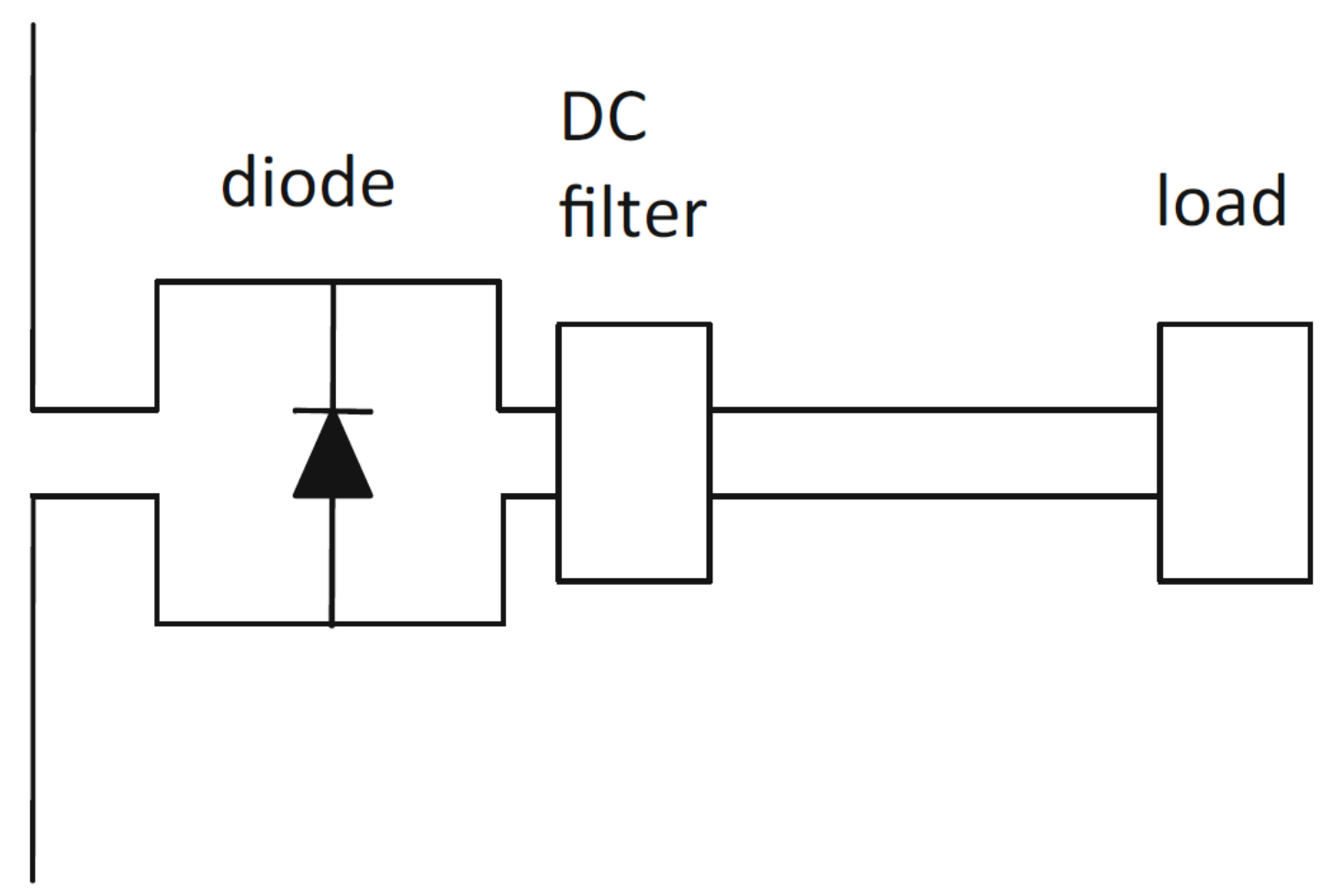

Figure 1.10: Circuit diagram of the rectenna system [5].

In terms of the antenna, there are a variety of structures that can simultaneously operate at different levels of power and in a wide frequency range, however, the rectifying circuit bandwidth is determined by several elements such as the matching circuit, the diode input impedance, and the load resistance $[53,54,55,56,57]$. To increase the harvested energy, rectennas that are working at multiple bands have been reported $[50,58]$. However, to operate at multiple frequencies, more cost and complexity are added to the rectenna's system.

Indeed, many designs have been proposed to operate in wide-range power levels and wide-band frequencies [59]. A design based on a dual-circular polarized spiral antenna array with a rectifying circuit was suggested by Hagerty et al., [59]. In this design, the operating frequency ranges from 2 to $18 \mathrm{GHz}$, and the input power ranges from 0.01 to $100 \mu \mathrm{W} / \mathrm{cm}^{2}$. The obtained AC to DC conversion efficiency is ranging from $0.1 \%$ to $20 \%$. 
This achievement is considered a good achievement since it overcomes several challenges. First, the level of the power of the ambient waves is very low. Second,the input impedance of the diode depends highly on the input power level. Third, the loss of the diode itself is very high at low power levels. Furthermore, other designs were proposed to harvest energy at wide-band frequency, but the matching circuit was still challenging [60, 61, 41]. Several configurations of wide-band antennas in rectenna systems have been presented in the form of a single antenna element $[62,46,63]$ and an antenna array $[64,65,66]$.

A rectenna system that harvests EM energy at 3 different frequency bands (tri-band rectenna) was proposed in [67]. A miniaturized multiband impedance matching circuit was utilized in the rectenna to operate at frequency bands centered at 24, 28, and $38 \mathrm{GHz}$. The simulated efficiencies of $44.3 \%, 42.7 \%$, and $43.6 \%$ respectively are obtained at an input power of $15.6 \mathrm{dBm}$. Whereas, the measured efficiencies at the same level of power were $35.3 \%, 31 \%$, and $30 \%$ respectively. Measurement results showed that increasing the input power increases the efficiency for all bands [67].

A cylindrical-shaped dual-band flexible rectenna system that is capable of harvesting RF energy from several RF sources coming from different directions is reported in [68]. To cover the radiation coming from different directions, four identical dual-band rectennas that are printed on a flexible substrate are utilized. Each rectenna comprises of a dual-band antenna and a dual-band rectifying circuit. To extract maximum power, these identical rectennas are printed on a cylindrical shaped filled by an artificial magnetic conductor. A maximum efficiency of $40 \%$ was attainable utilizing this design [68].

To avoid the dielectric loss, a wave*guide was utilized as a receiving antenna in a rectenna system instead of a microstrip antenna. A Fabry-Perot resonator that operates at $35 \mathrm{GHz}$ with a radiation efficiency of $95 \%$ is integrated with a rectifying circuit through a wave-guide-to-microstrip transition. An RF-to-dc efficiency of $68.5 \%$ is reported at a power density of $39.3 \mathrm{~mW} / \mathrm{cm}^{2}$ [69].

For the rectifying circuit, various design configurations have been suggested, such as half-wave rectifying circuit using shunt or series diode [70, 71], full-wave rectifier [72], and voltage doubler [73]. Of course, the full-wave configuration is more efficient than the half-wave one. However, channeling the power in the full-wave configuration is more challenging. For instance, a channeling mechanism for the design of dipoles array and a full-wave rectifying circuit was presented, where the rectifying circuit was connected between two dipoles [72]. The ripple of the output DC signal can be minimized by loading a series of inductive impedance or a parallel capacitive impedance with the load resistance $[63,39]$.

The rectenna system can be used in a power transmission link (point-to-point), where 
the design constraints of the rectenna can be tailored to the nature of the largest known (or predictable) incident field, including its frequency, polarization, and power density (see [74] as an example), or can be used to harvest ambient EM radiation over a specific frequency band (see [75, 76] as an example). In the latter application, where the rectenna acts as an energy scavenger since the incoming radiation is highly unpredictable in terms of frequency bandwidth, polarization, and power density, the design of the rectenna can be challenging. Harvesting the ambient EM radiation was investigated in urban and semi-urban areas. For instance, in [75], it was demonstrated that an ambient EM wave with a power density of $-25 \mathrm{dBm} / \mathrm{cm}^{2}$ can be harvested with $40 \%$ efficiency using a single band rectenna, and an ambient wave with a power density of $-29 \mathrm{dBm} / \mathrm{cm}^{2}$ can be harvested with a multi-band rectenna system. To evaluate the efficiency of a system with fluctuating levels of available power, in [75], the efficiency was calculated using energy instead of power.

Due to the diode's non-linearity, the input impedance of the diode is varying nonlinearly with the input power. The input impedance of the diode depends also on both frequency and load resistance [17]. To overcome the sensitivity of a rectenna system to the polarization, a circularly polarized rectenna system that operates at $5.8 \mathrm{GHz}$ is reported in [77]. To extend the stability of the design over a range of input power with an efficiency of more than $60 \%$, a parallel combination of two rectenna systems was suggested. The parallel connection of two identical rectifiers to the output of a hybrid coupler decreases the sensitivity of the whole system to the variation of the input power and enhances the stability of the system [77].

\subsection{Problem Statement}

\subsubsection{Problem One}

In general, improving component characterization accuracy leads to a high-performance design. Because the diode is the most important component in the rectifying circuit, an accurate nonlinear system model is necessary for optimal use of computer-aided design (CAD) technology. Many nonlinear device models today are made up of fundamental electric components including capacitors, inductors, and resistors. Building such models, on the other hand, necessitates complicated measuring processes to properly extract the relevant characteristics. Furthermore, developing and validating an appropriate model could take a year [78].

The scattering parameters (S-parameters) of a circuit are very useful parameters that can be used to design the matching networks in the radio frequency (RF) circuits. The 
S-parameters provide a relationship between incidental, transmitted, and reflected waves at each port of the circuit. S-parameters are only valid for describing linear devices. For non-linear devices, S-parameters are only valid when the input signal is a very small-signal, which leads to designs that have high efficiency at a specific power level and very narrowband frequency. In reality, S-parameters do not contain information about the harmonics that are generated by a non-linear device. Many attempts to generalize the S-parameters to be able to describe the nonlinear device are suggested, such as; hot S-parameter and largesignal S-parameter. These techniques have led to many problems, including the inability to obtain repeatable measurements [79].

Because of its non-linearity, the diode's S-parameters are dependent on the input power, which is one of the key obstacles in building a diode's circuit using its S-parameters. To overcome this problem, I can extract the diode's S-parameters at a specific level of input power and design the matching network according to these S-parameters. However, Sparameters are not able to fully describe a non-linear component. Figure 1.11 shows the response of a nonlinear device to a small signal and the response of a nonlinear device to a large signal. When the input signal is tiny, the nonlinear device behaves like a linear circuit, but when the input signal is large, it generates numerous harmonics, as seen in Figure 1.11. Instead of S-parameters, X-parameters are proposed to properly define the non-linear device. In reality, because they contain a complete description of the behavior of a non-linear device at the fundamental frequency and at all other harmonics, employing $\mathrm{X}$-Parameters is valid for linear and non-linear devices at any input power level and at any frequency. As a result, I can estimate the nonlinear device's output power at all harmonics based on its input power, allowing us to construct a rectifying circuit with a wide-band frequency and wide-range power. Furthermore, because X-Parameters can be extracted for a whole rectifying circuit, antenna tuning that best fits a certain rectifying circuit is achievable.

\subsubsection{Problem Two}

To maximize the efficiency of a rectenna circuit, I should increase the power transfer from the antenna's terminals to the input of the diode. This can be accomplished by incorporating a matching circuit between the antenna and the diode or the rectification circuit (such a matching circuit may be considered as part of the rectification circuit). The rectification circuit's input impedance is sensitive to fluctuations in input power due to its non-linearity. This fluctuation is a significant stumbling block to fully characterizing the rectenna system over its operating frequency range. Because of the significant fluctuation in input impedance with input power, the power profile at the antenna terminals must be 


\section{small-signal}

\section{linear output}
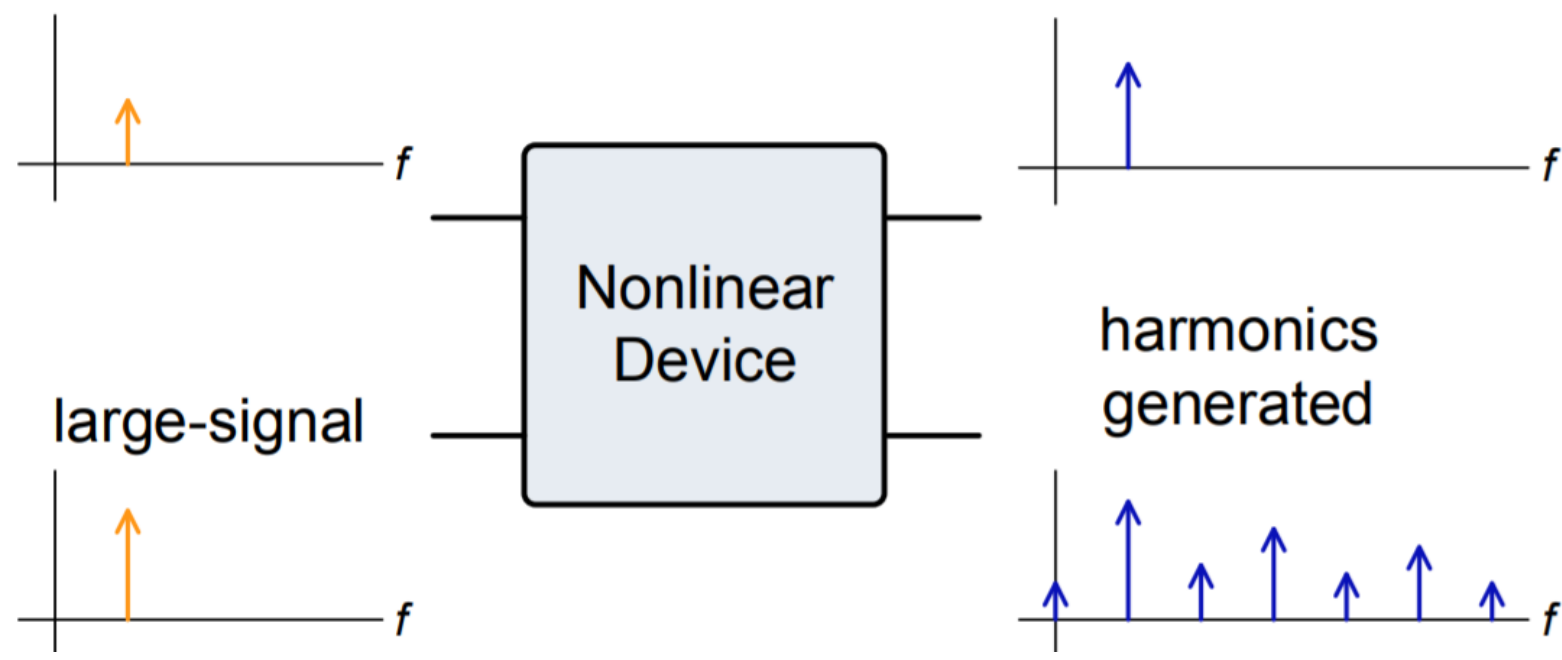

harmonics generated

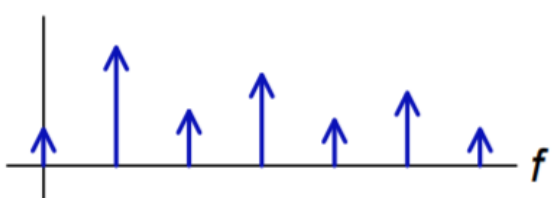

Figure 1.11: Responses of a nonlinear device to both small and large input signals.

incorporated in the modeling of the full rectenna system. The variation of the power level at the input of the rectification circuit would result in a large discrepancy between the simulation's results and the measurement's results if the power profile was not taken into consideration, as has been demonstrated in prior works [17, 80, 16].

Because the received power fluctuates with frequency, using a single power level across the entire frequency range would yield a precise result at only one frequency point, and limits our ability to precisely predict the result at other frequencies. It would also be difficult, if not impossible, to optimize a rectenna circuit over a wide frequency band. This necessitates utilizing an equivalent circuit that accurately characterizes the antenna part of the rectenna circuit. This circuit should account for the antenna's input impedance variations as well as the power fluctuation across its terminals as the frequency changes.

\subsubsection{Problem Three}

Recently, the growth of wireless technologies and applications requires antennas with multiple capabilities and functions. Characteristics like low cost, small size, high efficiency, and wide bandwidth are desired in many technologies. Furthermore, antennas that can be used both as flat and as curved (conformal antennas) are becoming an increasingly attractive antennas because of their wide range of application [81]. 
The microstrip patch antenna is one of the most widely used antennas, however it has a high sensitivity to bending. An antenna with a lower bending sensitivity is required to avoid the effect of bending on antennas. The metasurface antenna is one of the best candidates to overcome the problem of antenna's sensitivity to bending. To construct a metasurface, an ensemble of periodic resonators are structured to operate as an effective electromagnetic radiator. The efficiency of each resonator is extremely poor, however, due to the coupling between elements they, collectively, show good performance [82]. In MSA, the spacing between the radiating elements is very small and this necessitate the use of separated layer for the feeding network.

Unlike the MSA, each element of MPA array can operate effectively. To increase the gain of a single element MPA, several elements are utilized to form an MPA array [83]. To avoid coupling between the radiating elements in an MPA array, the distance between the elements should be equal to or larger than half wave length $(\lambda / 2)$.

\subsection{Contributions}

1. Because the rectifying circuit's major component (the diode) is a nonlinear device, a powerful technique that can handle nonlinear components is required. In nonlinear electronics, X-Parameters are a broad form of S-parameters that is suitable for both small and large signals. X-Parameters enable us to accurately characterize the nonlinear components and systems with multiple ports. X-Parameters are powerful mathematical tool that describes the behaviour of nonlinear components in response to large signals. The ability of utilizing X-parameters to characterize a diode and a complete rectenna system at different power levels and for various frequencies was validated in Chapter 3.

2. In chapter 4, I present an entirely simulation-based method to predict the performance of a complete rectenna system that includes all its components: the receiving antenna, the matching circuits between the antenna and the rectification circuit, and the load circuit. Whereas previous efforts to predict the performance of a rectenna system subdivided the system into the antenna part (radiation to $\mathrm{AC}$ power conversion) and the circuit part (AC power to DC power conversion), and made assumptions about the performance of the non-linear part of the rectenna based on a specified power level and frequency, in this method, the radiation part of the system is incorporated into the simulation by using Thevenin's theorem. The method proposed in this work enables the rectenna designer to predict the performance of the complete 
rectenna system, at the design stage, for variation in the incident field's power density, angle of incidence, and operating frequency. Furthermore, the proposed method enables the rectenna designers to optimize the entire system over sub-band or entire range of operating frequency. Experimental results are provided to demonstrate the accuracy of the method.

3. In chapter 5, I present a design of a Metasurface Antenna (MSA) utilizing electrical small elements to resonate strongly to resemble the resonance of classical circuits. Facilitated by matching due to coupling, the small radiators collectively provide a good radiation characteristics. The performance of the proposed MSA is compared with an MPA array design. Additionally, a rectenna system that utilize the MSA as receiving part is developed and investigated. Finally, the capability of the MSA to operate in the $5 \mathrm{G}$ band is investigated.

4. In chapter 6 , a novel dipole design with an adjustable input impedance is presented. The proposed dipole is designed to have additional arms attached at the edges of the feeding gap for the purpose of controlling the input impedance. Varying the dimensions of the two arms enables the tuning of the input admittance. This design is highly suitable for rectennas where the input impedance of the receiving antenna need to be matched to the input impedance of diodes which typically have a relatively high reactance. The design provides another benefit in recetenna design since it eliminates the use of matching circuits which can decrease the efficiency of the entire energy harvesting system. Numerical simulation results are provided to validate the design concept.

\subsection{Thesis Outline}

The thesis is divided into eight chapters. The following is how the rest of the thesis is organized:

Chapter Two begins with a review of a rectenna circuit, focusing on the components and attributes of a rectenna circuit. The processes for designing a rectenna circuit and the challenges that come with it are outlined in this chapter.

Chapter Three introduces the X-parameters along with their advantages. The nonlinearity of non-linear devices and their effects on design are explained in this chapter. This chapter presents X-parameters as an alternative for the circuit model to characterize a diode and a complete rectenna circuit. 
Chapter Four presents a method that integrates the electromagnetic part and the circuit part of the rectenna system to simulate and optimize the complete system. A complete rectenna system was modeled, built, and tested to validate the method. Good agreement between the simulated and the measured results are obtained.

Chapter Five proposes a novel metasurface antenna design that is optimized to operate in two frequency bands. The design was compared with a microstrip patch antenna. To validate the simulation results, the metasurface was modeled, fabricated, and tested. Good agreement between the simulated and the measured results are obtained. The metasurface was utilized to design a rectenna circuit.

Chapter Six introduces an antenna design that is matched to the nonlinear devices without using matching circuits by modifying the antenna parameters. In this Chapter, a controllable input impedance dipole antenna is designed. A parametric study was conducted on the design to validate the applicability of controlling the input impedance of the dipole. A rectifying circuit that is directly matched to the antenna was designed using the proposed design.

Chapter Seven concludes the achieved work and the future work. 


\section{Chapter 2}

\section{Rectennas}

\section{$2.1 \quad$ Introduction}

Energy harvesting comprises two stages; firstly, absorbing as much as possible of the incidental electromagnetic waves' energy, secondly, converting the absorbed AC energy into a useful DC energy as depicted in Figure 2.1. Observing Figure 2.1, the first stage can be considered as the electromagetic part of the system where the antenna acts as a transducer that converts the electromagnetic wave into an AC signal. The second stage is the rectifying circuit and it consists mainly of diodes, a matching circuit and a DC load. This chapter is devoted to discuss the components of the rectenna system and the associated challenges.

\subsection{EM Part}

The electromagnetic part consists of the received power and the receiving antenna. To mimic the behaviour of the EM part, I constructed the equivalent circuit shown in Figure 2.2 , where $P_{\text {received }}$ is the available power at the receiving-antenna's terminals and $Z_{\text {in }}$ is the internal impedance that corresponds to the receiving-antenna's impedance at different frequencies.

The available power $\left(P_{\text {received }}\right)$ can be calculated by [84]:

$$
P_{\text {received }}=P_{T} G_{T}(f)\left(\frac{A_{e f f}}{4 \pi R^{2}}\right)
$$




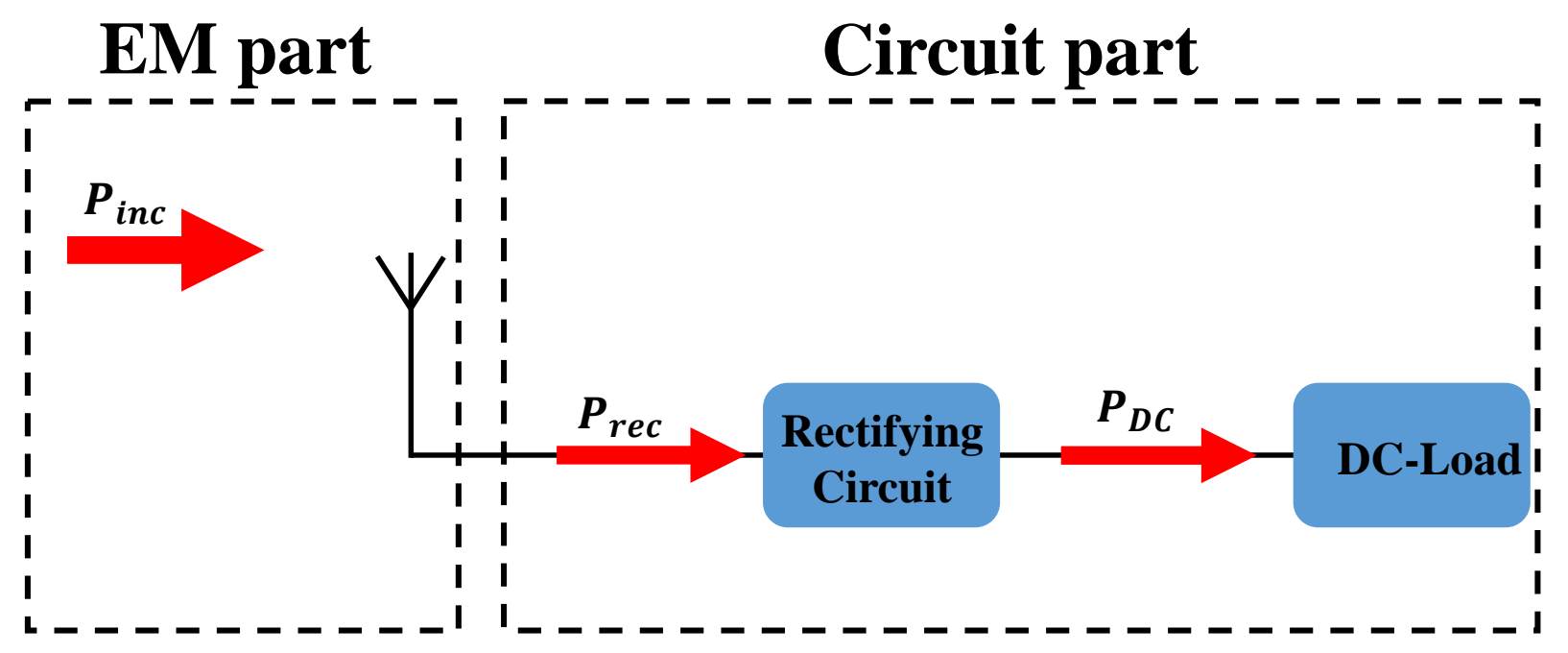

Figure 2.1: Rectenna system components.

where $P_{T}$ is the power that was fed to the transmitting antenna, $G_{T}(f)$ is the transmittingantenna's gain as a function of frequency and $\mathrm{R}$ is the separation distance between the transmitting and the receiving antennas. The effective area of an antenna $\left(A_{\text {eff }}\right)$ is defined as the ratio between the available power and the power density at its surface, and it can be calculated as:

$$
A_{e f f}=\frac{\lambda^{2}}{4 \pi} G_{R}=\frac{c^{2}}{f^{2}} \times \frac{G_{R}}{4 \pi}
$$

where $G_{R}$ is the receiving-antenna's gain and $\lambda$ is the wave length.

\subsection{Antenna}

Motivated by the theoretical prediction of Maxwell theory, Hertz experimentally demonstrated the existence of electromagnetic waves by the realization of the first antenna in 1888. The antenna is a transitional structure that acts as a transducer to convert electromagnetic waves into an AC signal (Receiver) or AC signal into electromagnetic waves (Transmitter). Several types of antennas have been utilized for wireless applications including, but not limited to, Microstrip Antennas (MAs), Metasurface Antennas (MSAs), 


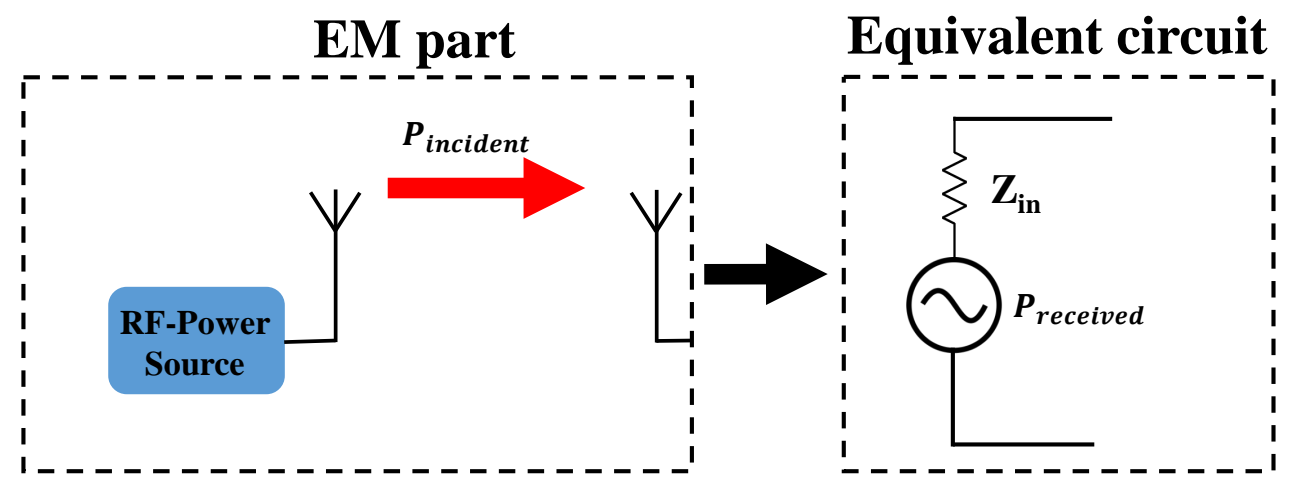

Figure 2.2: Equivalent circuit of the EM part.

wire antennas, reflector antennas, lens antennas, and aperture antennas. All the types of antennas can be compared and assessed based on common parameters and characteristics such as directivity, efficiency, gain, effective area, bandwidth, impedance, radiation pattern, polarization, and noise temperature. In the following subsections, I will discuss some important parameters and types of antennas.

\subsubsection{Antenna Parameters}

\section{Directivity}

In antenna, the maximum directivity $\left(D_{\max }\right)$ is defined as the ratio between the radiation intensity in the direction of maximum radiation $\left(U_{\max }\right)$ and the average radiation intensity in all directions $\left(U_{0}\right)$. The radiation intensity can be averaged over all directions by dividing the total radiated power by $4 \pi$. Mathematically the directivity can be calculated as [85]:

$$
D_{\max }=\frac{U_{\max }}{U_{0}}=\frac{4 \pi U_{\max }}{P_{\operatorname{rad}}}
$$

\section{Efficiency}

The efficiency of a transmitting antenna is defined as the ratio between radiated power and the power fed to the antenna's terminals from a perfectly matched source. Mismatching between the source and the antenna is separated from the efficiency of the antenna. The portion of the supplied power that is not radiated is dissipated as heat due to the loss 
resistance $\left(R_{\text {Loss }}\right)$ in the antenna's conductor. The antenna resistance can be divided into two types; radiation resistance $\left(R_{R a d}\right)$ and $R_{\text {Loss. }}$. If I provide an antenna's terminals with a current $I$, this antenna will radiate a power of $I^{2} R_{\text {Rad }}$ and will dissipate $I^{2} R_{\text {Loss }}$. Consequently, the efficiency of an antenna can be calculated as $\left(R_{R a d}\right) /\left(R_{R a d}+R_{L o s s}\right)$. Due to the principle of reciprocity, the efficiency of an antenna used as a receiver can be determined in the same manner as the transmitting antenna. Generally, the size of an antenna plays an important role in the efficiency of the antenna, and the antennas with a very small size compared with wavelength are inevitably inefficient [85].

\section{Gain}

Gain is one of the parameters that describe the performance of the antenna. The gain of an antenna takes into account both the directivity of the antenna, and the efficiency of the antenna and it is defined as the ratio between the power intensity, in a specific direction, and the power intensity given that all the power that is delivered to the antenna were isotropically radiated. This can be mathematically expressed as [85]:

$$
\text { Gain }=4 \pi \frac{\text { radiation intensity }}{\text { total input (accepted) power }}=4 \pi \frac{U(\theta, \phi)}{P_{\text {in }}}
$$

One of the useful terms is the relative gain which is defined as the ratio between the gain of an antenna in a specific direction and the gain of a reference antenna if they both were provided with the same power. Usually, the one uses a lossless isotropic source as a reference antenna, and the relative gain can be calculated as:

$$
G=\frac{4 \pi U(\theta, \phi)}{P_{i n}(\text { lossless isotropic source })}
$$

\section{Effective Area (Aperture)}

The effective area of a receiving antenna is the ratio between the power density available at the antenna and the power developed at the antenna terminals. Due to the sensitivity of the receiving antenna to the direction of the signal, the effective area of a receiving antenna is a function of the direction of the incidental wave. The effective area can be calculated using the following equation [85]:

$$
A_{\mathrm{eff}}=\frac{\lambda^{2}}{4 \pi} G
$$




\subsubsection{Antenna Types}

\section{Microstrip Patch Antenna (MPA)}

One of the most popular types that are used in wireless applications is the MA. MA's family is a compact antenna that has the capability of integration with a printed circuit. Because of their low cost, low profile, and many other advantages, MAs have attracted the attention of researchers. However, besides these advantages, MAs have disadvantages like low gain and narrow bandwidth. Table 2.1 illustrates some advantages and disadvantages of MAs.

Generally, an MA consists of a metallic radiating patch printed on a dielectric substrate that is backed by a ground plane (metallic layer) as shown in Fig. 2.3a. The concept of MA was first introduced by Deschamps in 1953 [86]. However, 20 years passed before a practical microstrip antenna was fabricated due to the lake of a substrate with a low loss tangent and good thermal properties during the 1950s. The first MA was fabricated by Howel and Munson [87, 88]. Extensive research has been done since the first MA was fabricated, and has led to advanced applications for the patch antenna. The metallic radiating patch can take several shapes; rectangular, triangular, ring, circular, etc. As a matter of fact, the shape of the metallic patch plays an important role in the antenna specifications such as; resonant frequency, polarization, input impedance, and radiation pattern. Some common shapes of the MAs are shown in Figure 2.3b.

The resonant frequency of a MA can be controlled by changing the dimensions of the patch and the relative permittivity of the substrate. For example, the resonant frequency of an equilateral triangle patch antenna with a side length of $a$ can be calculated using the following formula [89]:

$$
f_{m, n}=\frac{2 c}{3 a \sqrt{\epsilon_{r}}} \sqrt{m^{2}+m n+n^{2}}
$$

where $\mathrm{c}$ is the velocity of the light in free space, $m$ and $n$ are integers which are never zero simultaneously, $\left(f_{1,0}\right)$ is the fundamental frequency, and $\epsilon_{r}$ is the dielectric constant of substrate.

The MA, inherently, has a narrow bandwidth which is considered as a challenge for energy harvesting, where I need to harvest as much as possible of energy. Many techniques have been suggested to increase the bandwidth of the MA. One of the effective methods that enhance the bandwidth of MA is the decreasing of its quality factor. Decreasing the quality factor of an MA requires a thick substrate with a low dielectric constant (approximately 


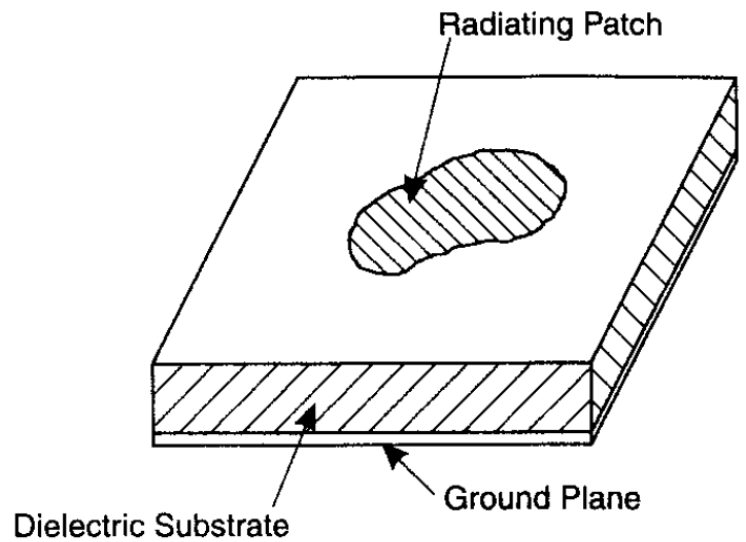

(a)
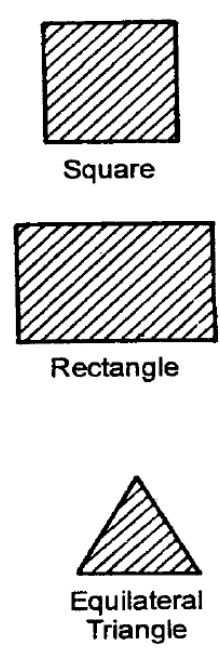

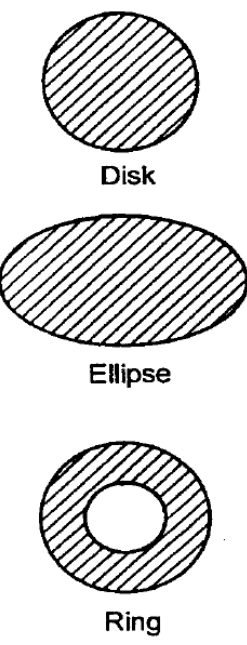

(b)

Figure 2.3: (a) General structure of a microstrip patch antenna [6]. (b) Common shapes of microstrip patch antenna [6].

unity). Due to the thick substrate layer, a large reactance is produced by a long probe feeding pin which limits the matching over a wide range of frequencies. To achieve matching over a wide range of frequencies, slots should be embedded on the patch [90].

Table 2.1: Advantages and disadvantages of MA.

\begin{tabular}{|c|c|}
\hline Advantages & Disadvantages \\
\hline Low profile & Narrow bandwidth \\
\hline Low cost & Low power handling \\
\hline Linear and circular polarization possibility & Low gain \\
\hline Dual band and dual polarization possibility & \\
\hline Feeding and matching fabricated within structure & \\
\hline
\end{tabular}

\section{Metasurface Antenna (MSA)}

A metasurface is a two-dimensional (2D) surface version of a three-dimensional volume (3D) metamaterial, with the surface consisting of a distribution of electrically tiny scatters, which was initially called meta film [91]. The metasurface is comprised of two-dimensional 
periodic structures made up of sub-wavelength scattering elements that reassemble incident waves to the desired level of transmission and reflection. The thickness and periodicity of metasurfaces are two significant elements of their structure, both of which are much less than the wavelength of the surrounding media. Because of the reduction in volume metamaterials, metasurfaces have the advantage of taking up less physical space than 3Dmetamaterial structures and are less-lossy structures. Additionally, they are light, simple to construct, flexible, and robust [92, 93] .

There are two forms of metasurfaces: the first is metafilm, which is made up of an array of isolated scatter elements that follow the cermet topology [91]. The second group is metascreen, which uses a fishnet architecture with periodically spaced apertures on the surface to organize the scattering elements. A homogeneous sheet with isolated aperture is referred to as a metascreen. Other types of metasurfaces can be defined based on a combination of these two extreme types, in addition to these two [94]. Figure 2.4 shows several types of materials, ranging from natural to man-made materials.

Because of all of these characteristics, metasurfaces have a wide range of applications. In addition to the previously described features, the metasurface particles are scalable, allowing them to be used in any frequency spectrum. Because of its adaptability, metasurface applications have sparked increased attention in the scientific and technical sectors in recent years. Absorbers [95, 96], harvesters [97], microwave radiation detectors [98], microwave-associated organic chemistry [99, 100], and fluid controlled surfaces [100] are only a few of the potential applications for metasurfaces.

\subsection{Diode}

Unlike the conventional PN-diodes, the Schottky diode is a junction between a metal and a doped semiconductor (P-type or N-type). In the Schottky diode, the flow of free electrons from a semiconductor to metal creates a depletion region across the junction due to the difference in energy levels (Schottky barrier) [7]. Figure 2.5 depicts the variation of both current and capacitance with applied voltage in both Schottky and conventional PN-diode. Observing the Figure, the forward bias voltage required to turn the Schottky diode on is less than that required to turn the conventional PN-diode on. On the other hand, the capacitance of the Schottky diode is much less than that of the conventional PN-diode. Practically, a lower threshold diode is more suitable for microwave energy harvesting because of its low power level. Additionally, a lower capacitance leads to highspeed switching, and this is required for high-frequency wave rectification. It can be noted 


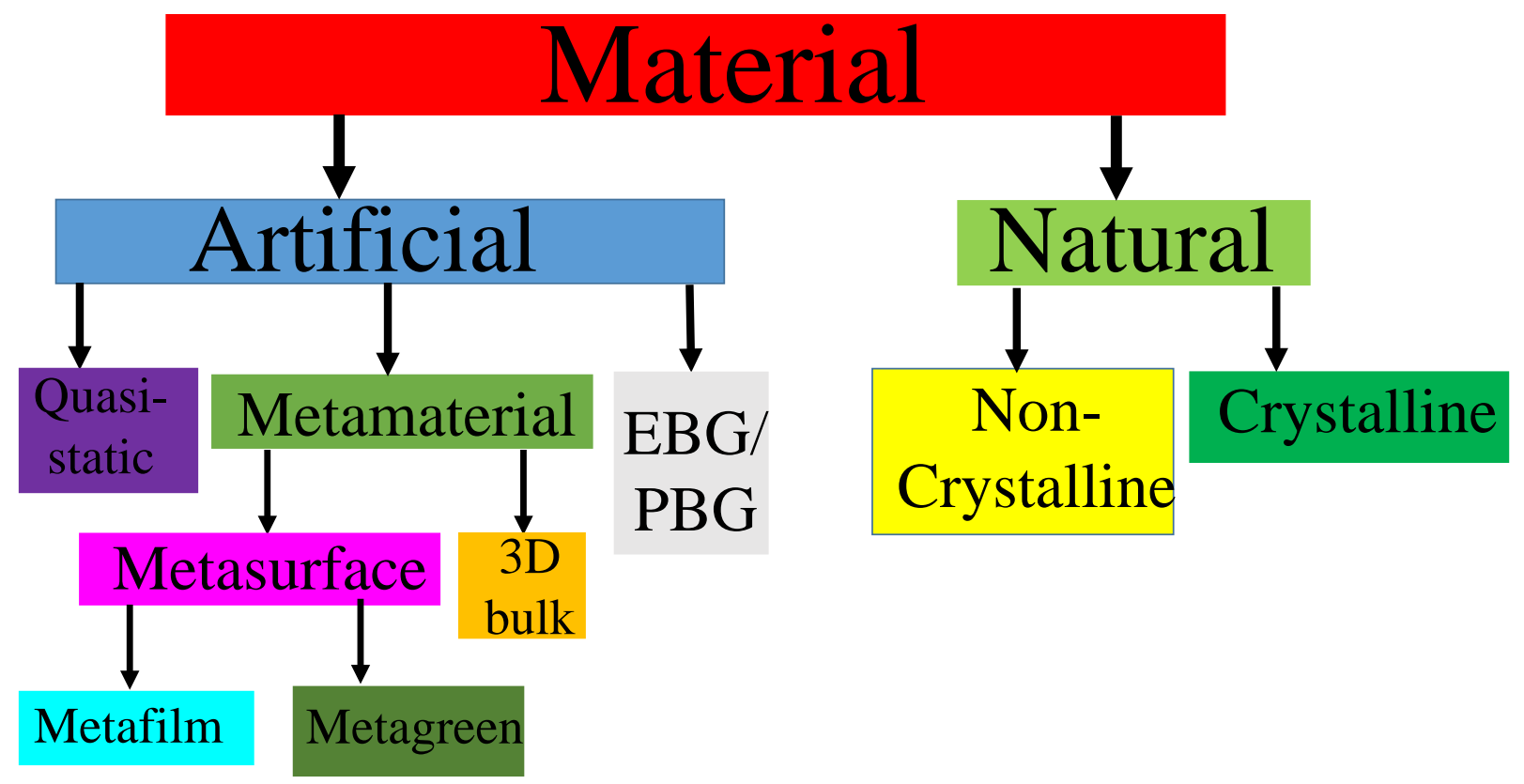

Figure 2.4: Classification of natural and artificial materials.

that the Schottky diode excels the conventional PN-diode in applications that require a very low turn-on voltage and high-speed switching like RF energy harvesting.

\subsubsection{Diode Model}

Figure 2.6 shows an equivalent circuit of a Schottky diode. As shown in the Figure, the resistance of the junction $\left(R_{j}\right)$ and the capacitance of the junction $\left(C_{d}\right)$ are varying with the applied voltage, consequently, the current variation of the diode is nonlinear with voltage. The Schottky diode is non-linear due to the non-linearity of $R_{j}$ and $C_{d}$. The series resistance $\left(R_{s}\right)$ is non-linear as well, but due to its small variation, I can consider it as a linear resistance [101]. The relationship between the current of the diode $\left(I_{d}\right)$ and the voltage of the diode $\left(V_{d}\right)$ is given by the Schottky equation:

$$
I_{d}=I_{s}\left(e^{\frac{q V_{d}}{N k T}}-1\right)
$$

where

$\mathrm{k}$ is the Boltzmann's constant. 

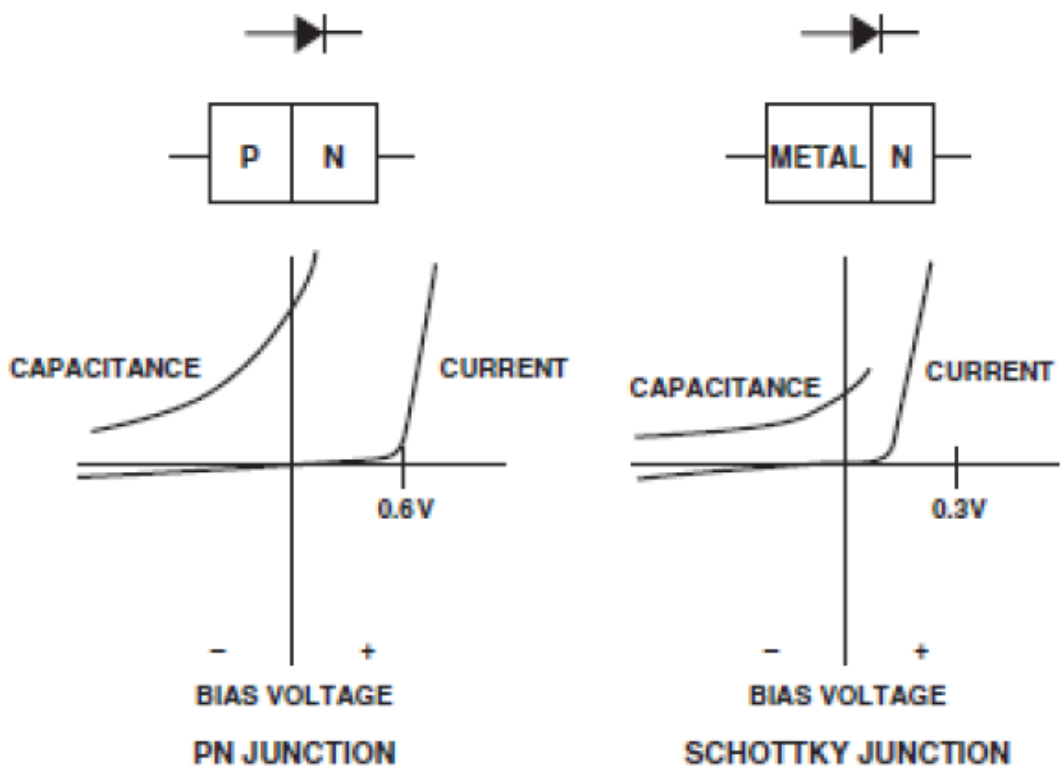

Figure 2.5: Current and capacitance variations with the applied voltage in both conventional PN-diode and Schottky diode [7].

$\mathrm{T}$ is the temperature in Kelvin.

$q$ is the charge of electron.

$\mathrm{n}$ is the quality factor (it is usually assumed to be 1).

$I_{s}$ is the saturation current (scale current).

If I substitute $\alpha=\mathrm{q} / \mathrm{NkT}$, then the equation 2.8 become:

$$
I_{d}=I_{s}\left(e^{\alpha V_{d}}-1\right)
$$

If I assume that, a sinusoidal RF signal with a maximum voltage of $V_{0}$ drives the diode then:

$$
V_{d}=V_{0} \cos (\omega t)
$$




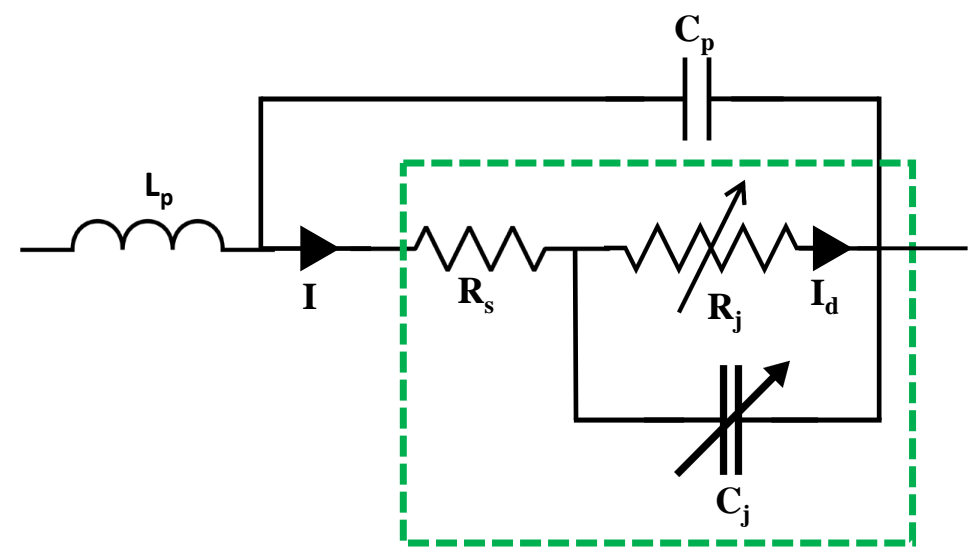

Figure 2.6: Equivalent circuit model of a diode; the components outside the green box are parasitic components.

Using Taylor series I obtain $I_{d}$ as [102]:

$$
\begin{array}{r}
I_{d}=\frac{\alpha^{2} I_{s} V_{0}^{2}}{2 \cdot 2 !}+\frac{3 \cdot \alpha^{4} I_{s} V_{0}^{4}}{8 \cdot 4 !}+\left(\alpha I_{s} V_{0}+\frac{3 \cdot \alpha^{3} I_{s} V_{0}^{3}}{4 \cdot 3 !}\right) \cos (\omega t)+\left(\frac{\alpha^{2} I_{s} V_{0}^{2}}{2 \cdot 2 !}+\frac{\alpha^{4} I_{s} V_{0}^{4}}{2 \cdot 4 !}\right) \cos (2 \omega t) \\
+\frac{\alpha^{3} I_{s} V_{0}^{3}}{4 \cdot 3 !} \cos (3 \omega t)+\frac{\alpha^{4} I_{s} V_{0}^{4}}{8 \cdot 4 !} \cos (4 \omega t) \cdots
\end{array}
$$

Equation 2.9 shows that the diode's currents are distributed at fundamental frequency, harmonic frequencies, and at DC. Consequently, to obtain a high quality rectifying circuit, the fundamental and harmonic frequencies should be blocked by a suitable band pass filter (BPF) between antenna and the diode. Moreover, a low pass filter (LPF) should be used between diode and the load to eliminate the ripple.

To investigate the harmonics of a diode, I utilized the harmonic balance in Advance Design System (ADS) simulator to construct the circuit illustrated in Fig. 2.7 which comprises a power source, an HSMS-2860 diode [103], and a load of $50 \Omega$ resistance [104]. I adjusted the input power source to $10 \mathrm{dBm}$ and the operating frequency at $1 \mathrm{GHz}$, then I simulated the first five harmonics of the fundamental frequency. Figure 2.8 (a) and (b) depict the load current and voltage respectively due to the input power at different harmonics. The magnitude of the current ranges from almost zero at $5^{\text {th }}$ harmonic to 7.8 $\mathrm{mA}$ at the fundamental frequency, The magnitude of the voltage ranges from almost zero at $5^{\text {th }}$ harmonic to $0.39 \mathrm{~V}$ at the fundamental frequency. 


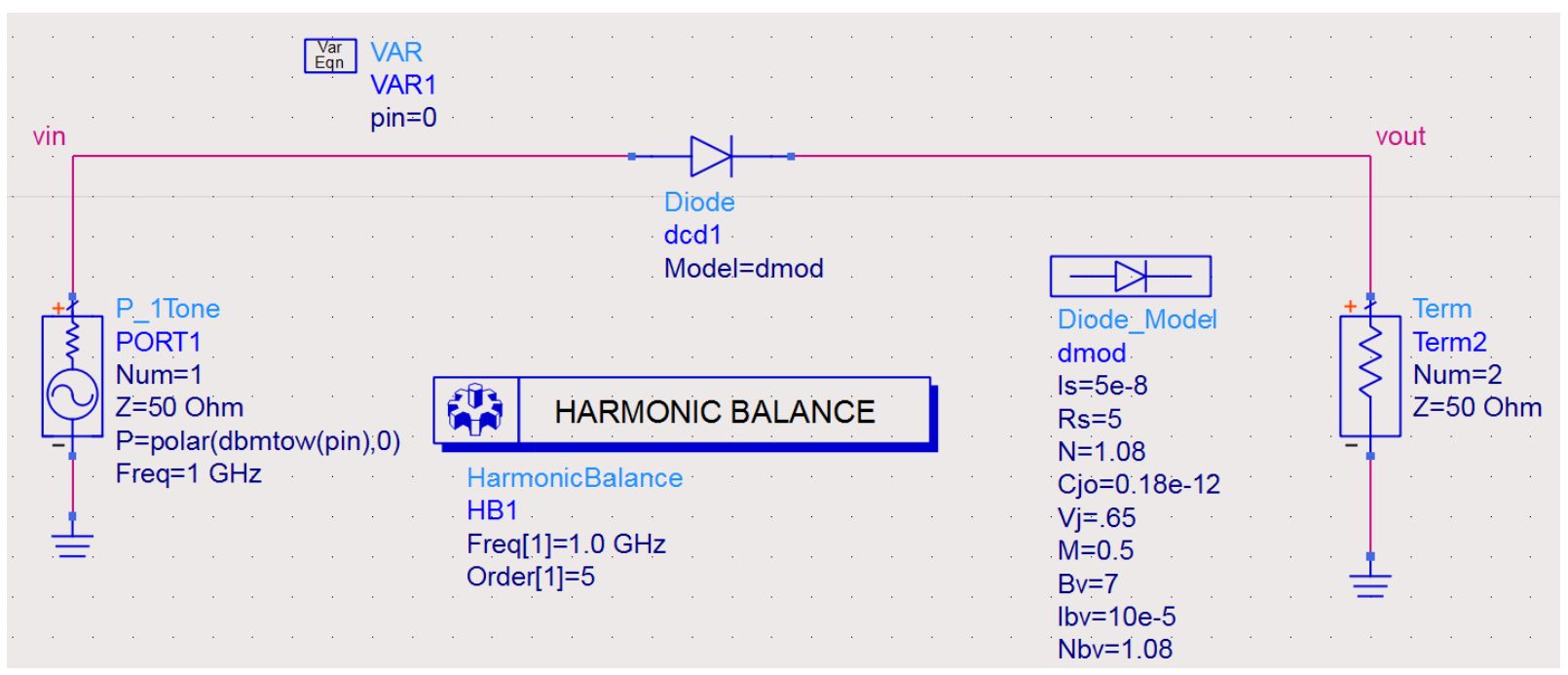

Figure 2.7: Schematic circuit of a diode with two ports.

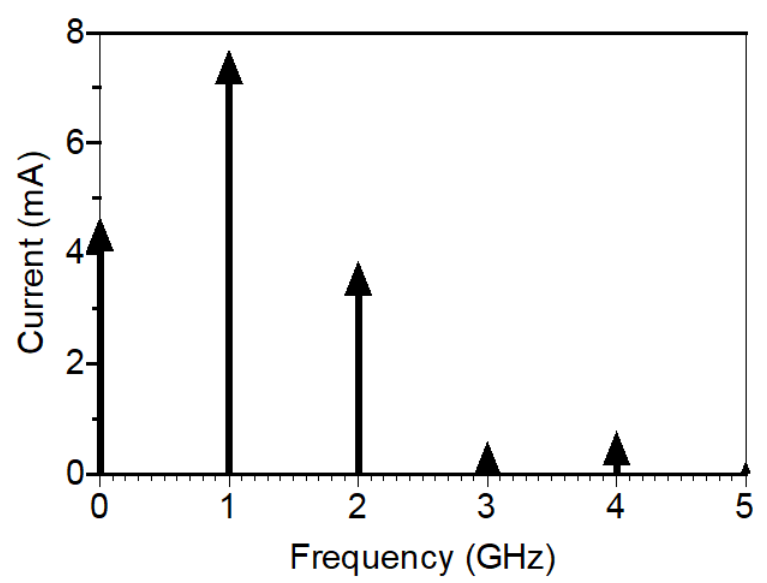

(a)

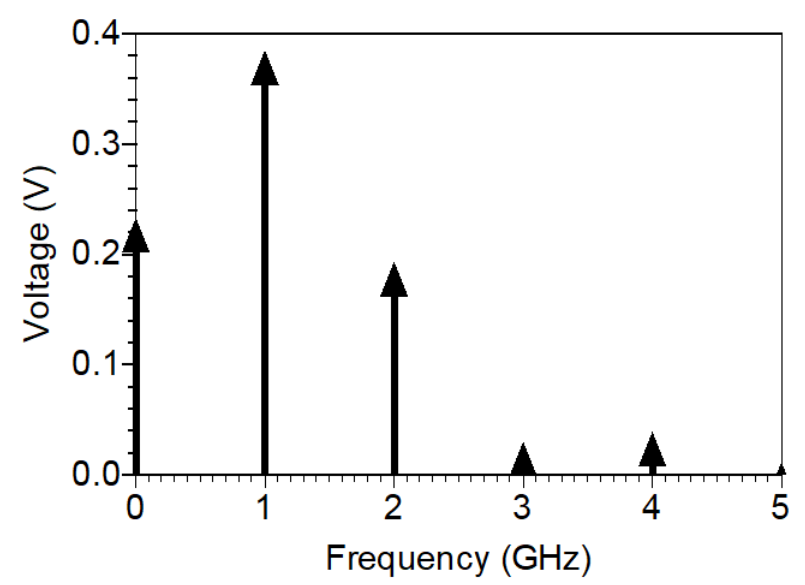

(b)

Figure 2.8: Output harmonics due to $10 \mathrm{dBm}$ input power: (a) Output currents of HSMS2860 at different harmonics. (b) Output voltage of HSMS2860 at different harmonics. 


\subsubsection{Parameters of Schottky Diodes}

Diode plays an important role in rectifying circuits, and this necessitates choosing an appropriate diode to maximize the efficiency of the rectifying circuit. Indeed, there are several types and vendors of diodes in the market. However, Schottky diodes are considered as one of the best candidates for rectenna system applications due to their high relatively switching speed and low turn-on voltage. To explore the behavior of the diode, I shed light on some parameters that affect diode performance. Table 2.2 shows some parameters of Schottky diode HSMS-28xx series. These parameters are explained in the following subsections.

\section{Junction potential $\left(V_{j}\right)$}

Junction potential represents the turn-on voltage at which a diode approximately acts as a short circuit in the forward bias. To maximize the sensitivity of a diode, the junction potential should be as small as possible such that any relatively small voltage can turn the diode on. Observing Table 2.2, the HSMS-2850 has the minimum junction potential among all of HSMS-28xx series with a voltage of $0.35 \mathrm{~V}$.

\section{Zero-bias junction capacitance $\left(C_{j 0}\right)$}

Due to the accumulation of opposite charges in both regions of the diode, the diode shows a capacitance between its terminals. This capacitance depends strongly on the applied voltage and the zero-bias junction capacitance. Zero-bias junction capacitance is the initial capacitance of the diode without the application of voltages. The capacitance of the diode can be determined by the following equation:

$$
C_{d}=C_{j 0}\left(1-\frac{V_{d}}{V_{j}}\right)^{-M}
$$

where

$V_{d}$ is the applied voltage.

$\mathrm{M}$ is the grading coefficient.

As a matter of fact, the capacitance of the diode limits the operating frequency of the diode. Increasing the capacitance of the diode decreases the speed of switching between on and off state, consequently, the diodes of lower capacitance is more suitable for high 
frequency applications. Table 2.2 shows the zero-bias junction capacitance of some of the HSMS-28xx series in pF. As illustrated in the Table, HSMS-2850 and HSMS-2860 diodes show the minimum zero-bias junction capacitance with capacitance of $0.18 \mathrm{pF}$.

\section{Diode series resistance $\left(R_{s}\right)$}

Diode series resistance is a crystal resistance of the diode that depends on the dimensions and the material of the diode. Unlike the resistance of conventional resistors, the diode series resistance is nonlinear. This means that the relation between the applied voltage and the current pass through the diode is nonlinear, however, due to its small variation, it can be considered as a constant. Table 2.2 illustrates the values of series resistance of different type of HSMS-28xx series. It can be noted the HSMS-2860 diode shows the minimum resistance amongst the other types with only $5 \Omega$ resistance. The series resistance of a diode can be calculated by:

$$
R_{s}=\frac{L}{q \mu_{n} N_{D} A}
$$

where

$\mu_{n}$ is the mobility of electrons.

$\mathrm{L}$ is the length of the diode.

$N_{D}$ is the doping density (atoms $/ \mathrm{cm}^{3}$ ).

$\mathrm{q}$ is the electron's charge.

\section{Breakdown voltage $\left(B_{v}\right)$}

Breakdown voltage is the maximum voltage that the diode can tolerate before it becomes conducting appreciably in reverse bias. The breakdown voltage determines the maximum output DC voltage of the rectifier and it should be taken into account when designing a rectifying circuit. The values of breakdown voltage of HSMS-28xx series are depicted in Table 2.2.

\section{Saturation current $\left(I_{s}\right)$}

Saturation current represents the thermal current from metal to semiconductor and it depends on the height of the diode barrier[105]. Increasing the barrier's height increases the forward voltage drop, which decreases the sensitivity of the diode. Conversely, decreasing 
the barrier's height increases the reverse leakage current. Opposite to a conventional PN junction, Schottky diode shows a low barrier height at forward bias and high barrier height at reverse bias.

Table 2.2: Parameters of Schotkky diode HSMS-28xx series [8].

\begin{tabular}{|c|c|c|c|c|}
\hline Diode & HSMS-2810 & HSMS-2820 & HSMS-2850 & HSMS-2860 \\
\hline$V_{j}(V)$ & 0.65 & 0.65 & 0.35 & 0.65 \\
\hline$C_{j 0}(p F)$ & 1.1 & 0.7 & 0.18 & 0.18 \\
\hline$R_{s}(\Omega)$ & 10 & 6 & 25 & 5 \\
\hline$B_{v}(V)$ & 25 & 15 & 3.8 & 7 \\
\hline$I_{B V}(\mu A)$ & 10 & 100 & 100 & 10 \\
\hline$I_{s}(n A)$ & 4.8 & 22 & 3000 & 50 \\
\hline Frequency & $\mathrm{RF}$ & $\mathrm{RF}$ & $<1.5 \mathrm{GHz}$ & $915 \mathrm{MHz}-5.8 \mathrm{GHz}$ \\
\hline
\end{tabular}

\subsection{Matching Circuit}

The matching network is defined as a circuit that matches the impedance between two points. In the rectenna circuit, due to the mismatching between the antenna's terminals and the rectifying circuit, the matching circuit plays an important role to maximize the efficiency of the whole system. Figure 2.1 shows the power components that may appear in a basic rectifying circuit. In Figure 2.1, $P_{i n c}$ represents the total coming power from RF source; $P_{r e c}$ is the power delivered to the input of the rectifying circuit; $P_{D C}$ is the output DC power. The radiation-to-DC power efficiency $\left(\eta_{\text {rad-DC }}\right)$ of a rectenna is defined as the ratio of the output DC power $\left(P_{D C}\right)$ to the total input power $\left(P_{\text {inc }}\right)$. To maximize the efficiency of a rectenna, the power delivered to the diode input terminal should be as much as possible by designing an efficient matching circuit. Mathematically, $\eta_{\text {rad }-D C}$ of a rectenna system can be expressed as[105]:

$$
\eta_{\text {rad-DC }}=\frac{P_{D C}}{P_{\text {inc }}} \times 100 \%=\frac{V_{D C}^{2} / R_{L}}{P_{\text {inc }}} \times 100 \%
$$

\subsubsection{Steps of Designing a Matching Circuit Utilizing S-parameters}

Due to the non-linear relation between the current and voltage of the diode, the Sparameters of the diode strongly depend on the power level and the operating frequency. 
Conventionally, designers extract the S-parameters of a diode at a single point of frequency and a single level of power. Utilizing the ADS numerical simulator, I was able to extract the S-parameters of HSMS-2860 diode, and utilize them to design a matching circuit based on the extracted S-parameters yet at a very narrow band. This section is devoted to explaining the steps of designing a matching circuit between the antenna and the diode utilizing S-parameters.

\section{Extracting S-parameters of a Diode}

To investigate the variation of the S-parameters of a diode with frequency and power, I utilized the ADS simulator to construct the circuit shown in Fig. 2.9. The circuit is composed of two ports with an internal resistance of $50 \Omega$ for each, a model of HSMS-2860 Schottky diode provided by the vendor. In this simulation, I utilized a Large Signal S Parameter (LSSP) simulation controller in ADS. To be able to sweep the power level and the operating frequency, I assigned the variable $P_{i n}$ to the input power and the variable LSSP freq to frequency as depicted in the circuit. LSSP simulation controller performs harmonic balance simulation and enables us to sweep any variable in the circuit. To study

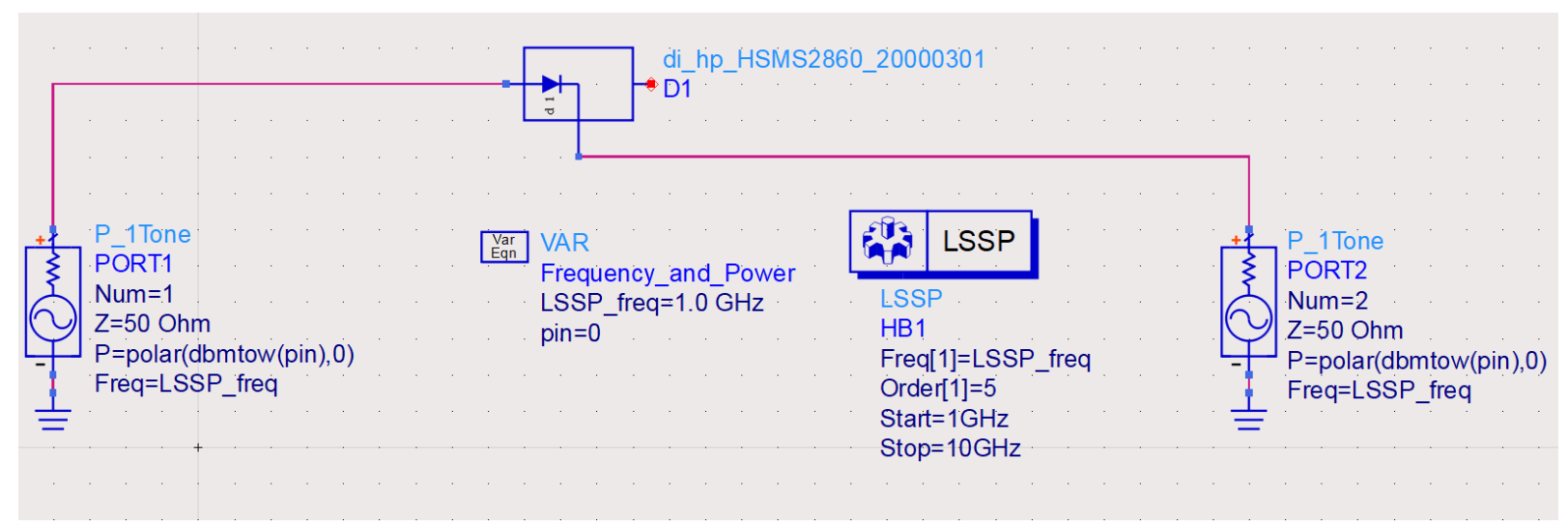

Figure 2.9: Schematic circuit of a diode with two ports to extract S-parameters.

the variation of the reflection coefficient of the diode with the frequency, I fixed the source power at $0 \mathrm{dBm}$ and swept the operating frequency from 1 to $10 \mathrm{GHz}$. As illustrated in Figure 2.10, the reflection coefficient of the diode is dramatically changing with frequency. The reflection coefficient of the diode is ranging from $-15 \mathrm{~dB}$ at $8 \mathrm{GHz}$ to approximately $-2.1 \mathrm{~dB}$ at $1 \mathrm{GHz}$. 


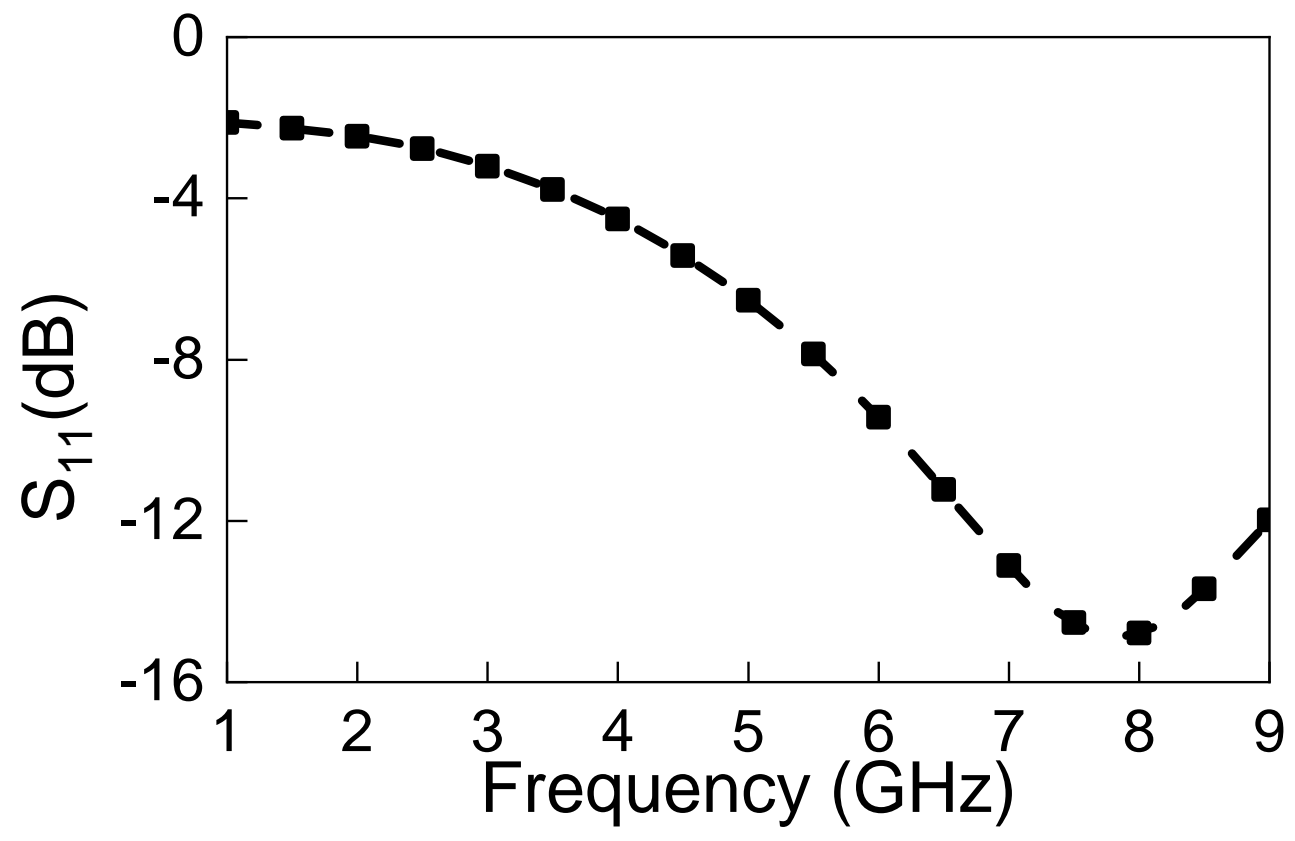

Figure 2.10: Variation of the reflection coefficient (S11) of the HSMS-2860 diode with frequency and at a fixed input power of $0 \mathrm{dBm}$.

To study the variation of the reflection coefficient of the diode with the input power, I fixed the frequency at $2 \mathrm{GHz}$ and swept the power of the source from $-40 \mathrm{dBm}$ to 40 $\mathrm{dBm}$. Observing Figure 2.11, the reflection coefficient of the diode is almost constant for input power ranging from -40 to $-10 \mathrm{dBm}$, however, the reflection coefficient of the diode dramatically changes with input power ranging from -10 to $40 \mathrm{dBm}$. The reflection coefficient of the diode is ranging from $-0.5 \mathrm{~dB}$ at $-10 \mathrm{dBm}$ input power to $-12.3 \mathrm{~dB}$ at 40 $\mathrm{dBm}$. It can be noted that, for the power values larger than $-10 \mathrm{dBm}$, the values of the reflection coefficient decrease as I increases the input power.

\section{Designing a Matching Circuit Using the ADS Simulator}

To design the matching circuit of the rectifying circuit, I can utilize the smith chart matching tool provided by ADS. Smith chart tool enables us to add transmission lines, open stubs, and short stubs to reach the matching situation. Designing a matching circuit utilizing the smith chart tool requires the availability of S-parameters of the diode. For instant, to design a matching circuit at a frequency of $2 \mathrm{GHz}$ and power of $0 \mathrm{dBm}$, I considered the value of $S_{11}$ at $0 \mathrm{dBm}$ power level and $2 \mathrm{GHz}$ frequency which is equal to $0.73-\mathrm{j} 0.187$ 


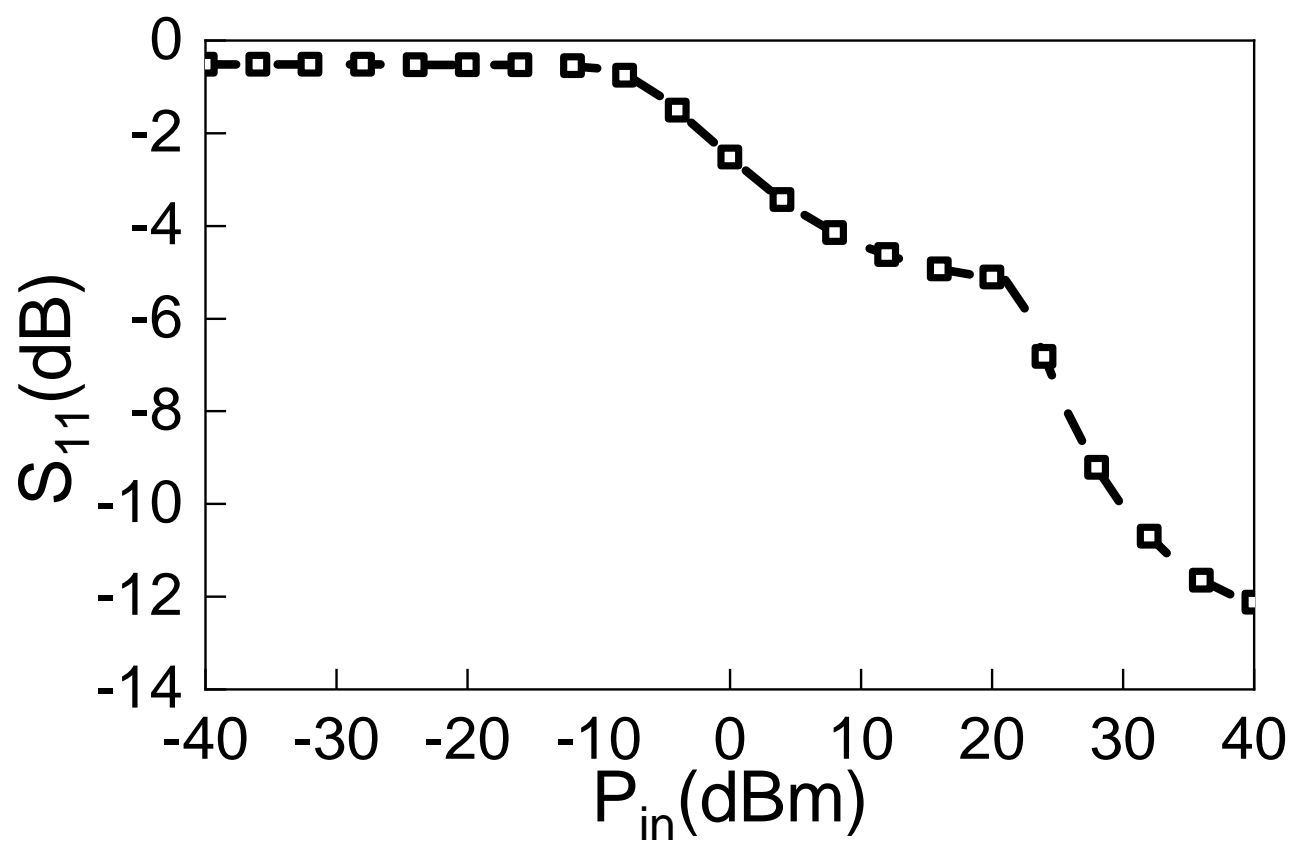

Figure 2.11: Variation of the reflection coefficient (S11) of the HSMS-2860 diode with input power and at a fixed operating frequency of $1 \mathrm{GHz}$.

as illustrated in Figure 2.12. Figure 2.13 shows the obtained matching circuit with all its parameters. As illustrated in the Figure, the matching circuit compromises two open stubs and two transmission lines printed on a material of $1.52 \mathrm{~mm}$ thickness (h) with a dielectric constant of 3.48 .

Figure 2.14 illustrates the schematic circuit of the rectenna system which compromises a diode, the matching circuit that is designed to operate around $2 \mathrm{GHz}$, and two $50 \Omega$ ports. The reflection coefficient of the complete circuit is shown in Figure 2.15. As illustrated in the Figure, the reflection coefficient is lower than $-10 \mathrm{dBm}$ for the frequencies ranging from 1.89 to $2.2 \mathrm{GHz}$. 


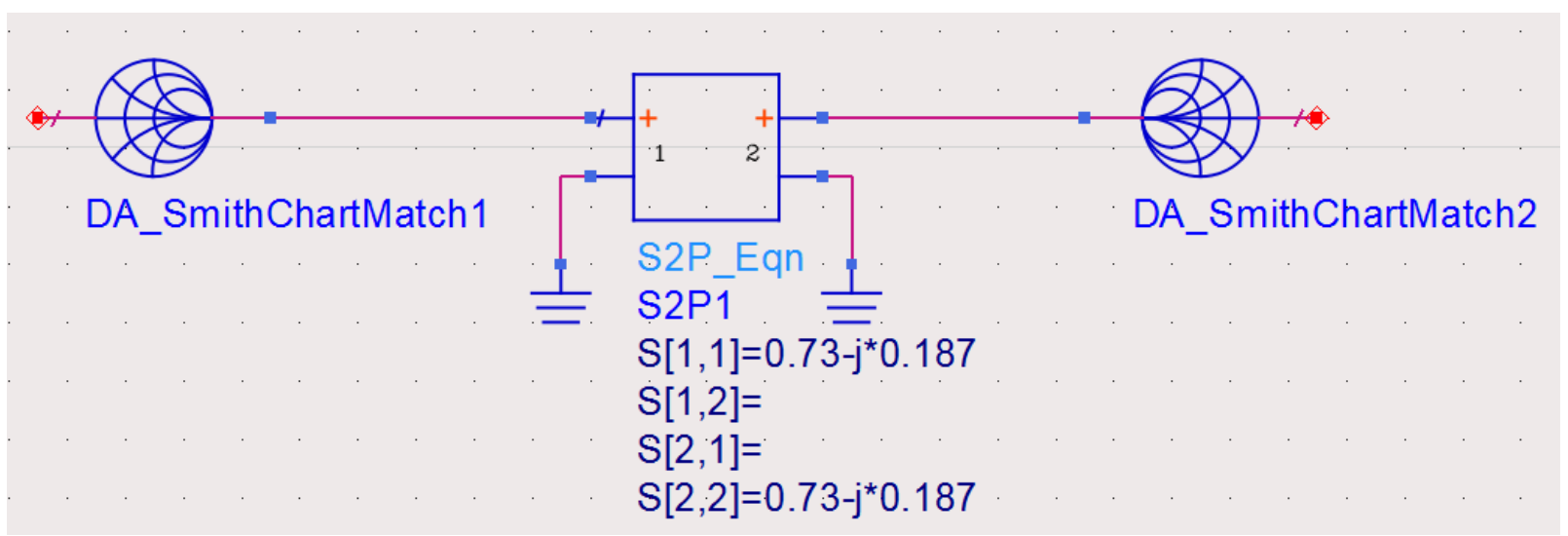

Figure 2.12: Smith chart tool used for designing a matching in ADS with the values of $S_{11}$.

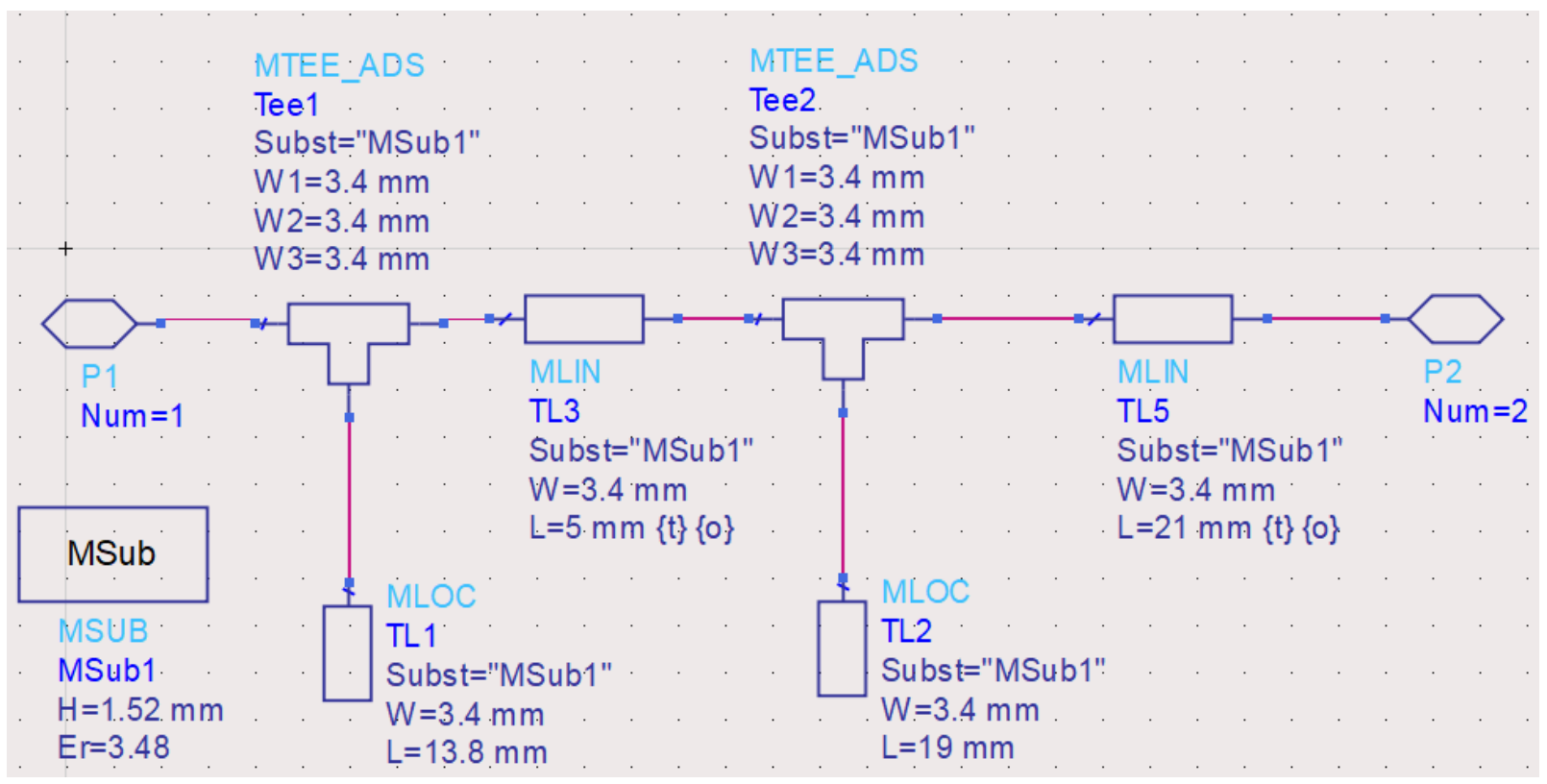

Figure 2.13: Schematic of the obtained matching circuit utilizing smith chart. 


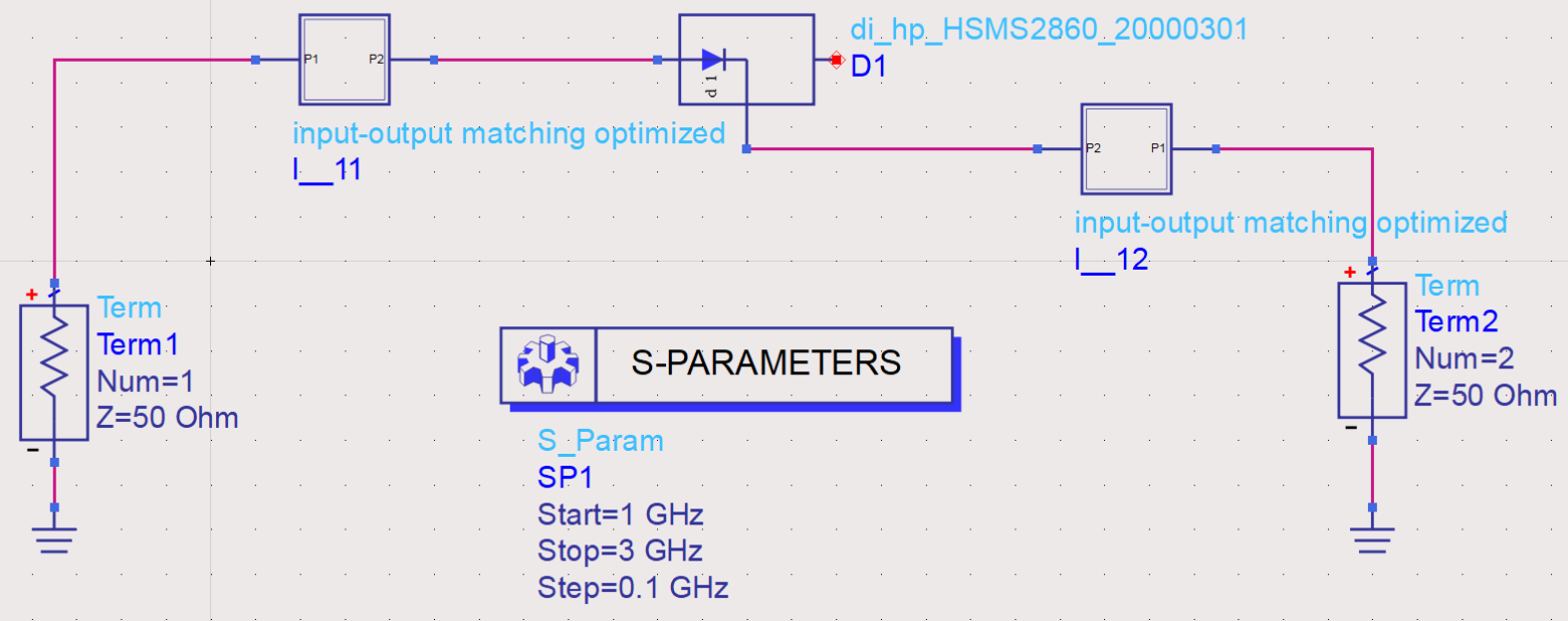

Figure 2.14: Schematic circuit of the diode and the matching circuit.

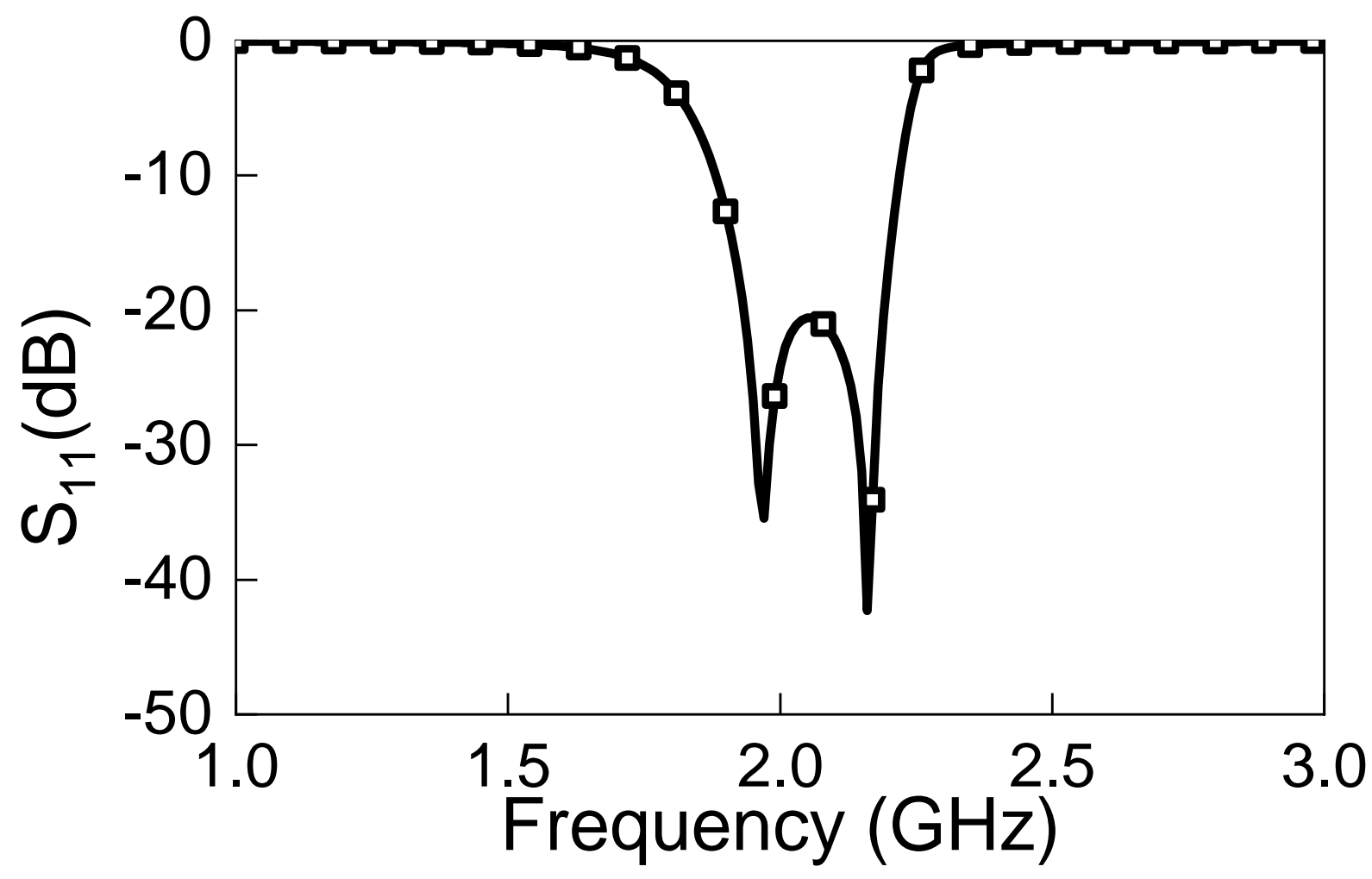

Figure 2.15: Simulated reflection coefficient of the rectifying circuit. 


\subsection{Challenges Associated With Designing Rectenna System}

Designers face many challenges when designing a rectenna system, including, but not limited to, the non-linearity of the diode, dependency of the available power at the terminal of antenna on frequency and the angle of incidence, and the variation of the input impedance of antenna on frequency. The non-linearity of a diode makes the input impedance of the rectenna circuit sensitive to any variation in power level/frequency. These related variations limit the ability of designers to design an efficient matching circuit. This means that when constructing a rectifier, it's critical to minimize the effects of these variations on the rectifier's performance by choosing a rectifier architecture that's insensitive to changes in power and load. Obviously, to properly simulate a rectenna system, the variation of any factor that affects the system like the frequency, the power level and the input impedance should be taken into consideration.

In order to imitate the fluctuation of the diode's input impedance with input power level at different frequencies, I built the circuit illustrated in Fig. 2.16. As illustrated in the Figure, it consists of a voltage source, a diode and a load resistance. In this circuit, I utilized HSMS-2860 diode nonlinear model, and I loaded its parameters from its data sheet as depicted in the Figure. I utilized the Harmonic balancing controller in ADS to model the circuit. The voltage source is an independent voltage source and its values can be swept by the harmonic balance controller. The load resistance is fixed at $50 \Omega$.

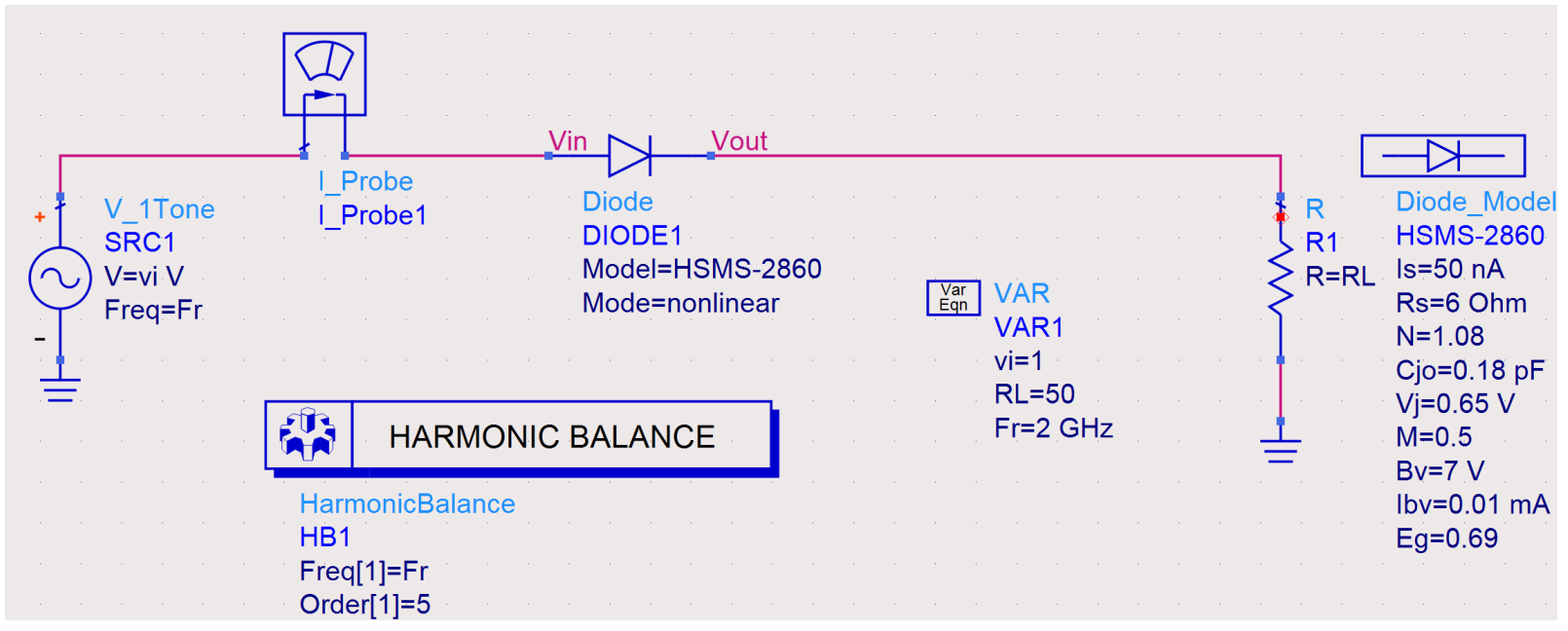

Figure 2.16: Schematic circuit used to simulate the input impedance of the diode. 
The input impedance of the diode is defined as the ratio between the voltage drop across the diode and the input current. To obtain the voltage drop across the diode, I defined two voltage nodes at the diode's terminals (Vin and Vout) as illustrated in the Figure. To simulate the input current, I utilized current probe (I_Probe) defined in ADS as shown in the schematic circuit. To turn on the diode, the values of the voltage source was swept from $0 \mathrm{~V}$ to $2 \mathrm{~V}$ with a voltage step of $0.01 \mathrm{~V}$. At fundamental frequency, the input impedance is defined as an equation in the ADS as following:

$$
Z_{\text {in }}=\frac{(\operatorname{Vin}[::, 1]-\operatorname{Vout}[\because:, 1])}{I_{-} \operatorname{Probe} 1 . i[\because:, 1]}
$$

where Vin[::,1] is the input voltage (Vin) over all sweeping range at harmonic 1 (fundamental frequency), Vout[::1] is the output voltage (Vout) over all sweeping range at fundamental frequency, and I_Probe1.i[::,1] is the values of current over all sweeping range at fundamental frequency.

Figure 2.17 shows the variation of the input impedance of the diode for 3 values of frequencies $(1.5 \mathrm{GHz}, 2 \mathrm{GHz}$ and $2.5 \mathrm{GHz})$. Observing the Figure, the real part and the imaginary part of the input impedance change dramatically with the input voltage ranging from 0 to $1 \mathrm{~V}$ at each frequency. Furthermore, the input impedance of the diode is also changing with frequency for the same voltage value.

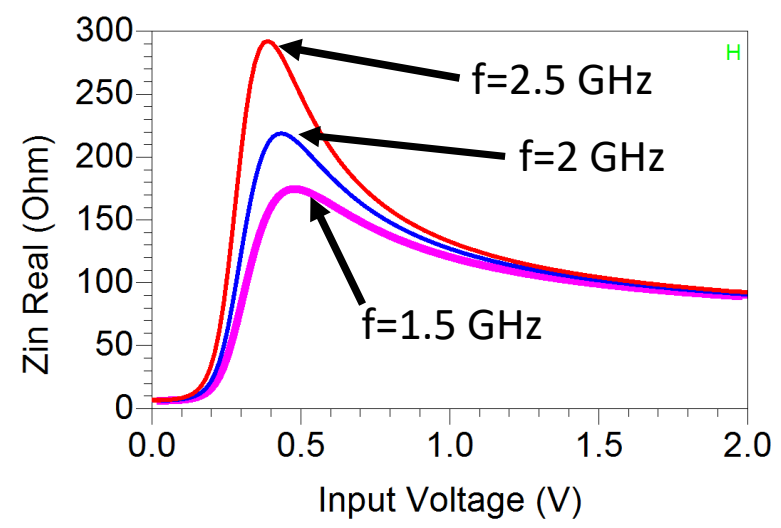

(a)

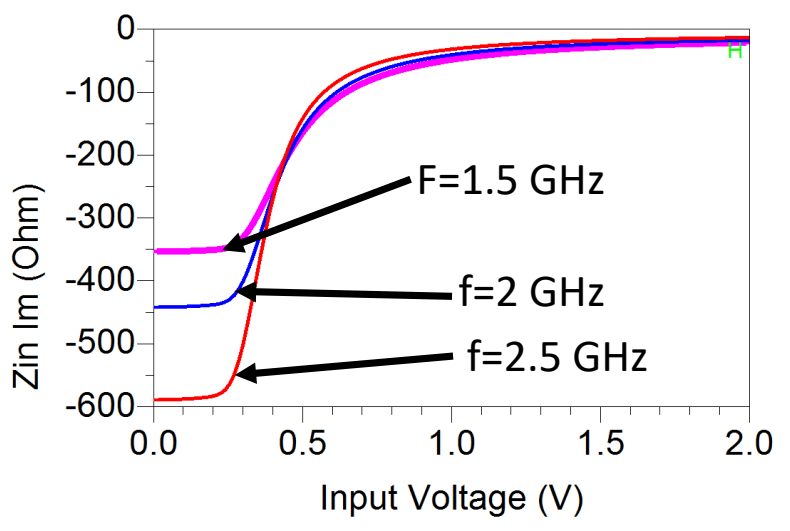

(b)

Figure 2.17: Variation of diode's input impedance with input voltage for different values of frequencies; (a) Real part (b) Imaginary part. 


\subsection{Conclusion}

This chapter covers the antenna, diode, and matchin circuit, which are the three essential components of a rectenna system. In the antenna section, I went through some of the key parameters to consider when designing an antenna. I also presented two different types of antennas, the Patch antenna and the Metasurface antenna. In the diode part, the equivalent circuit and non-linearity of the diode were explained. I also compared different types of Schottky diodes. The steps of designing a matching circuit were covered, and a simulation example was presented in the matching section. Finally, I discussed the challenges associated with designing a rectenna system. 


\section{Chapter 3}

\section{X-Parameters in Rectenna's Designs}

\subsection{Introduction}

Because the rectifying circuit's major component (the diode) is a nonlinear device, a powerful technique that can handle nonlinear components is required. In non-linear electronics, X-Parameters are a broad form of S-parameters that is suitable for both small and large signals. X-Parameters enable us to accurately characterize the nonlinear components and systems with multiple ports. X-Parameters are powerful mathematical tool that describes the behaviour of nonlinear components in response to large signals. The difference in response of a nonlinear device to small and large signal is illustrated in Fig. 3.1.

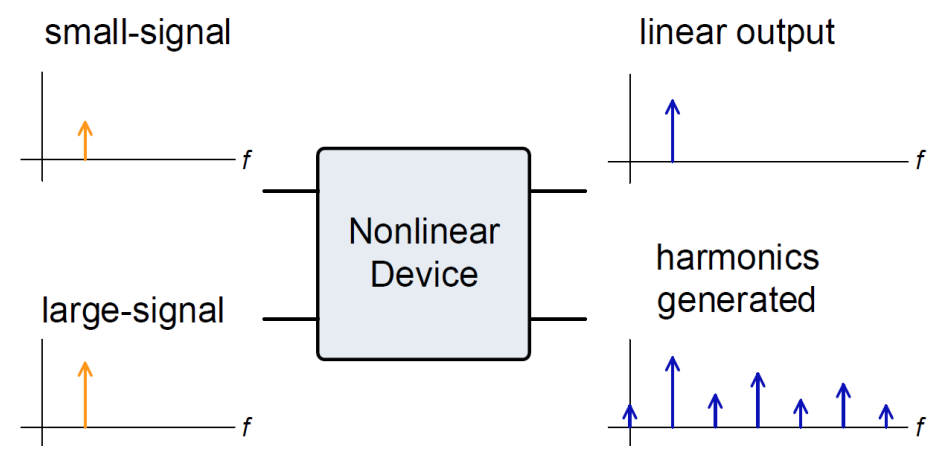

Figure 3.1: Difference in response of a nonlinear device to small and large signal.

As shown in the Figure, X-Parameters reduce exactly to S-parameters in the small signal, and they have the same simple use model as S-parameters. The key strength of 
the X-Parameters is that they contain information about all the harmonics and intermodulation spectra generated in response to large signals. Figure 3.2 shows a two-ports network with its inputs and outputs.

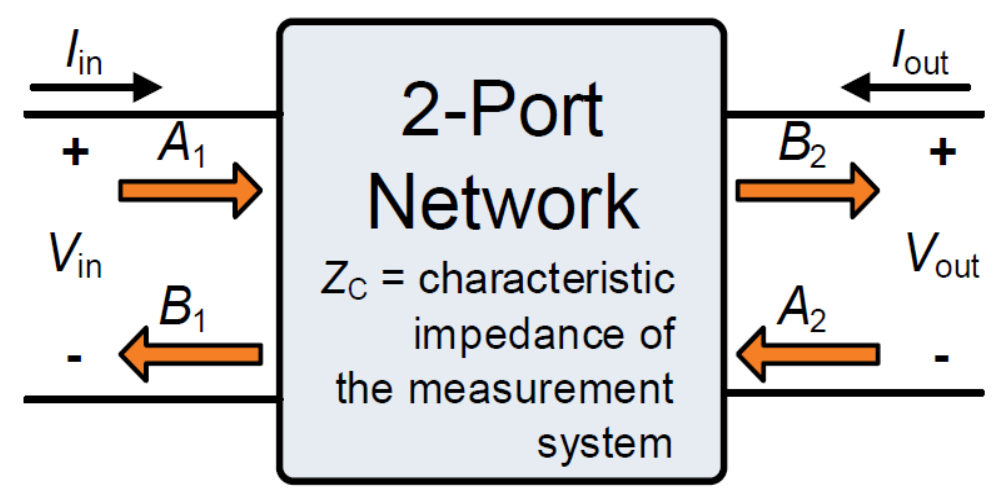

Figure 3.2: Two ports network with its input and output.

In this Figure, the waves $A_{1}, A_{2}, B_{1}$ and $B_{2}$ are represented, respectively, by:

$$
\begin{gathered}
A_{1}=\frac{\left(V_{\text {in }}+Z_{C} l_{\text {in }}\right)}{2 \sqrt{Z_{c}}} \\
A_{2}=\frac{\left(V_{\text {ott }}+Z_{C} l_{\text {out }}\right)}{2 \sqrt{Z_{c}}} \\
B_{1}=\frac{\left(V_{\text {in }}-Z_{c} l_{\text {in }}\right)}{2 \sqrt{Z_{c}}} \\
B_{2}=\frac{\left(V_{\text {out }}-Z_{c} l_{\text {out }}\right)}{2 \sqrt{Z_{c}}}
\end{gathered}
$$

In fact, the relationship between waves can be expressed as [79]:

$$
\mathrm{B}_{e, f}=X_{e f}^{(F)}\left(\left|A_{11}\right|\right) P^{f}+\sum_{g, h} X_{e f g h}^{(S)}\left(\left|A_{11}\right|\right) P^{f-h} \cdot A_{g h}+\sum_{g, h} X_{\epsilon f g h}^{(T)}\left(\left|A_{11}\right|\right) \quad P^{f+h} \cdot A_{g h}^{*}
$$

where, the indices; e is the output port, $\mathrm{f}$ is the output frequency harmonic, $\mathrm{g}$ is the input port index and $\mathrm{h}$ is the input frequency harmonic. The phase $\mathrm{P}$ is introduced by: 


$$
P=e^{j \cdot p h a s e\left(A_{1,1}\right)}
$$

In equation 3.5 the X-Parameters split into 3 terms: $X^{(F)}, X^{(S)}$ and $X^{(T)} . X^{(F)}$ represents the harmonic spectrum in response to the incident signal, on other words, $X^{(F)}$ term measures the nonlinear device behaviour at 50 ohms, when I inject a signal $A_{11}$ with a perfect match and with no harmonics. $X^{(S)}$ shows the effect of a mismatch at specific harmonic on the performance at another harmonic. In fact, $X^{(S)}$ is equivalent to the hot S-parameters, which was an initial attempt to extend S-parameters to nonlinear behavior. In hot S-parameters, I inject a large signal to the input port and a small signal to the output port with a small offset frequency and measure the reflected waves, but it was found that this technique is not efficient under highly nonlinear condition. The $X^{(T)}$ provides the information about the relationship between the mismatch phase at the ports and the fundamental and harmonic frequencies. Indeed, $X^{(T)}$ term accurately accounts for the highly nonlinear higher-order mixing products between the output and the input which are not captured in hot S-parameters. In fact, $X^{(F)}, X^{(S)}$ and $X^{(T)}$ rely on the frequency, the power relations between ports, and optionally on the bias.

\subsubsection{Advantages of X-Parameters}

It is very advantageous to use X-parameters when I do not have an accurate model for the diode. Indeed, the vendors provide us a table model with the impedance of the device extracted at specific frequency and specific power level. Whereas, X-parameters enable us to measure the device's nonlinear characteristics at all frequencies and all power levels. The following are some advantages of X-parameters [106]:

1. A measured X-Parameters are more accurate than a compact model, specifically, for large signal within a specific range of operating frequency. In fact, many compact models neglect some physical effects in large signal RF application, which leads to the non-accuracy of the model. Whereas, when I measure the X-Parameters, I get what the device does.

2. Measuring the X-Parameters is much easier than extracting a compact model for a nonlinear device. For instance, an advanced compact model may contain large number of parameters, and it needs an experienced device modeling engineer to obtain a good model. Whereas, extracting X-Parameters needs much less time and can be done by a technician.

3. The accuracy of the model depends on how the model was extracted, even the good model may be not accurate if it was poorly extracted. 
4. X-Parameters consider, directly, all the effects of packaging and coupling, whereas, in compact model I need a bare model (compact model), a model for the package, and a model for coupling if it is exist.

\subsection{Comparison Between X-Parameters and Circuit Model}

In general, increasing the accuracy of components' characterization leads to a high performance design. Since the main component in the rectifying circuit is the diode, an accurate model for nonlinear system is required for efficient use of computer aided design (CAD) technology. Currently, many models of the nonlinear devices consist of basic electric elements such as; capacitors, inductors, and resistors. However, building such models needs complex measurement procedures to extract the required parameters precisely. Furthermore, it may take a year to develop and validate an accurate model [78].

In light of challenges associated with traditional approaches of modeling, an accurate and relatively easy method based on X-Parameters to model nonlinear devices has been presented. The X-Parameters based model can be extracted by measurements, utilizing nonlinear vector network analyzer (NVNA), or by simulation using commercial simulation tools [107]. Furthermore, X-Parameters can be measured at different frequencies and at different power levels and stored at one file. The availability of all information at one file enables us to characterize the systems that operate under unstable conditions, such as the rectenna circuits that are used for energy harvesting. In this section, I describe the process of utilizing X-Parameters to simulate a nonlinear device/circuit. The obtained results are compared with the results obtained by circuit model.

\subsubsection{Simulation of Diode Using X-Parameters}

To verify the validity of X-Parameters to mimic the circuit model of a diode, I extracted the X-Parameters of a Schottky diode using X-Parameter module in ADS. Then, I implemented X-Parameter block on the same software in a "Harmonic Balance" module. The outcome of this simulation allows us to observe the component's temporal function and hence determine whether the extracted X-Parameters are capable of mimicking device behavior.

Initially, the approach is applied to a schottky diode (HSMS-2860). The layout of the simulation, that is used to extract X-Parameters, is depicted in Fig. 3.3. In the X- 
Parameters module, the larger the order "harmonic" number, the more precise will be the model. A compromise between the simulation time and the precision of the model should be made. It is found that the power transported by harmonics beyond the $4^{\text {th }}$ harmonic was negligible. Thus, in my simulation I take the first four harmonics $\left(5^{\text {th }}\right.$ order) into consideration. The parameter of the frequency is assigned to a variable "Fre". This variable is used in the "Sweep Plan" module to vary the frequency values from $1 \mathrm{MHz}$ to $4 \mathrm{GHz}$ in 10 points distributed linearly.

Input port of NVNA is represented by the module "XP_source" as illustrated in Fig. 3.3. Utilizing the "Parameter Sweep" module I swept the frequency (Fre) in this demonstration from $1 \mathrm{GHz}$ to $4 \mathrm{GHz}$. The power values are varied from $-20 \mathrm{dBm}$ to $20 \mathrm{dBm}$ with 10 dBm step. These values are assigned to the variables "LS_start (1, Mag)" and "LS_stop (1, Mag)". The second port of NVNA is represented by the "XP_Load" module whose impedance value characteristic is fixed at $50 \Omega$.

Once the simulation is finished, the X-Parameters will be stored in the data folder. Then, I open a new page of simulation in which I utilize the "Harmonic Balance" (HB) module as displayed in Fig. 3.4. The goal of this simulation is to compare the results obtained by simulating the diode circuit model with the results obtained by simulating the diode utilizing X-Parameters. The X-Parameters block shown in Fig. 3.4 is loaded with the data file which contain the generated X-Parameters in the utilizing the circuit in Fig. 3.3 .

In Fig. 3.4, the voltage difference between the diode's terminals (Vout-Vin) in the upper part of the schematic is defined as $\mathrm{Vd}$. The voltage $(\mathrm{Vx})$ is the voltage difference between the X-Parameters block's terminals (Vout_x-Vin_x) in the lower part of the schematic in Fig. 3.4.

The generated data contains 4 frequency points and 5 power levels for each circuit. However, to be able to compare between the circuit model and X-Parameters model, I fixed the operating frequency at $1 \mathrm{GHz}$ and the power level at $20 \mathrm{dBm}$. The simulated values of $\mathrm{Vx}$ at the first 5 harmonics are illustrated in Fig. 3.5a as red arrows, and the simulated values of $\mathrm{Vd}$ at the first 5 harmonics are illustrated in Fig. 3.5a as black arrows.

As depicted in the Figure, there are 6 harmonic indices, the harmonic 0 represents the DC component, the fundamental frequency corresponds to index 1 and the following indices are the harmonics of the fundamental frequency. To be able to differentiate between the results, I made the red arrows thicker than the black ones. On a given harmonic index, an arrow represents the value of the voltage at a specific frequency point and a specific power level. The simulation results show a good agreement between the X-Parameters model and the circuit model. The agreement concludes that the X-Parameters model can replicate 


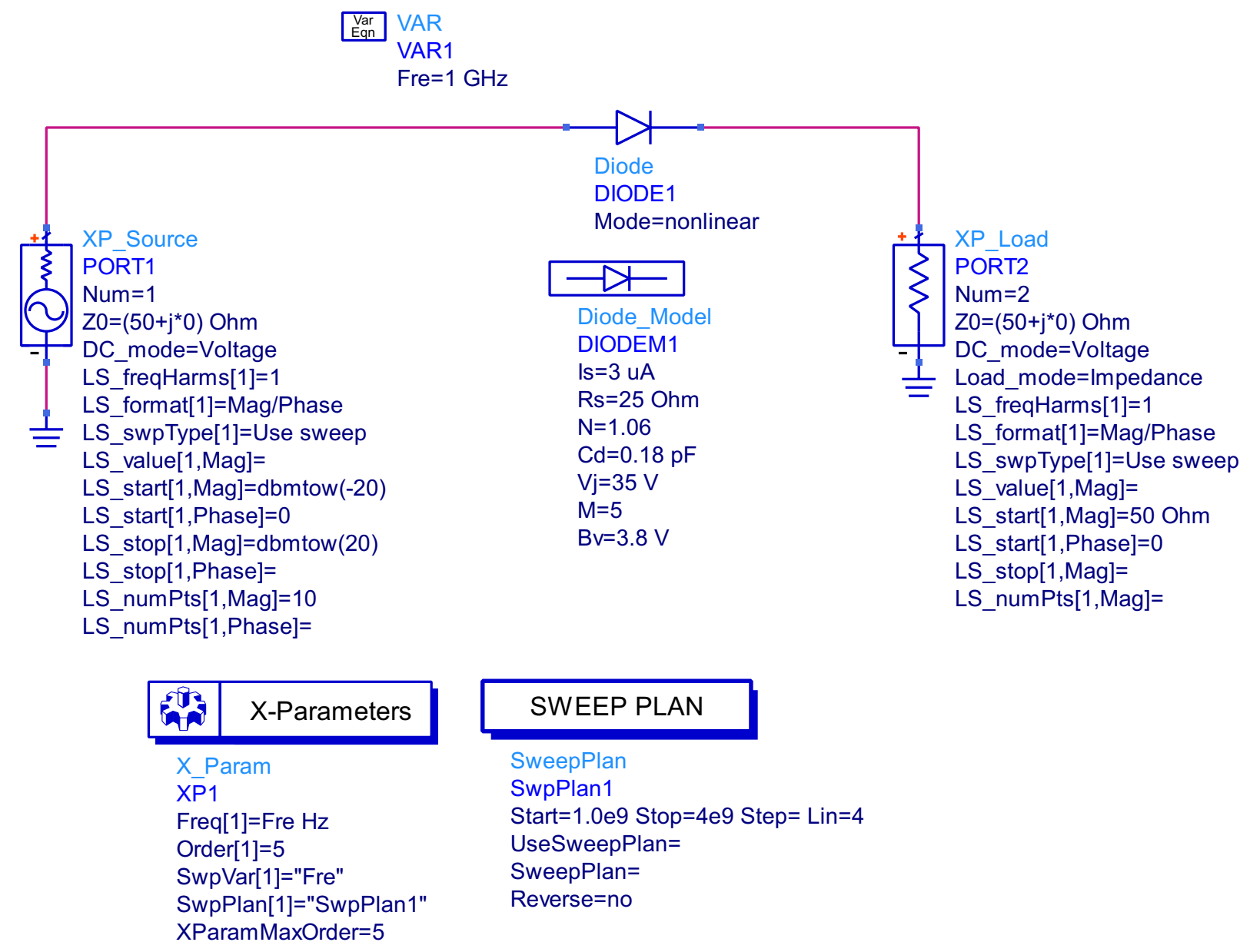

Figure 3.3: X-Parameters extraction simulation layout.

the results obtained by circuit model. Figure 3.5b shows the current passes through the diode and the current passes through the block of X-Parameters. It can be noted, the results obtained from the circuit model and the X-Parameters model are identical.

\subsubsection{Simulation of a Complete Rectenna Utilizing X-Parameters}

\section{Antenna Design}

As a receiving side, I utilized a Microstrip Patch Antenna (MPA), which consists of a metallic radiating patch printed on a dielectric substrate and backed by a ground plane. The 

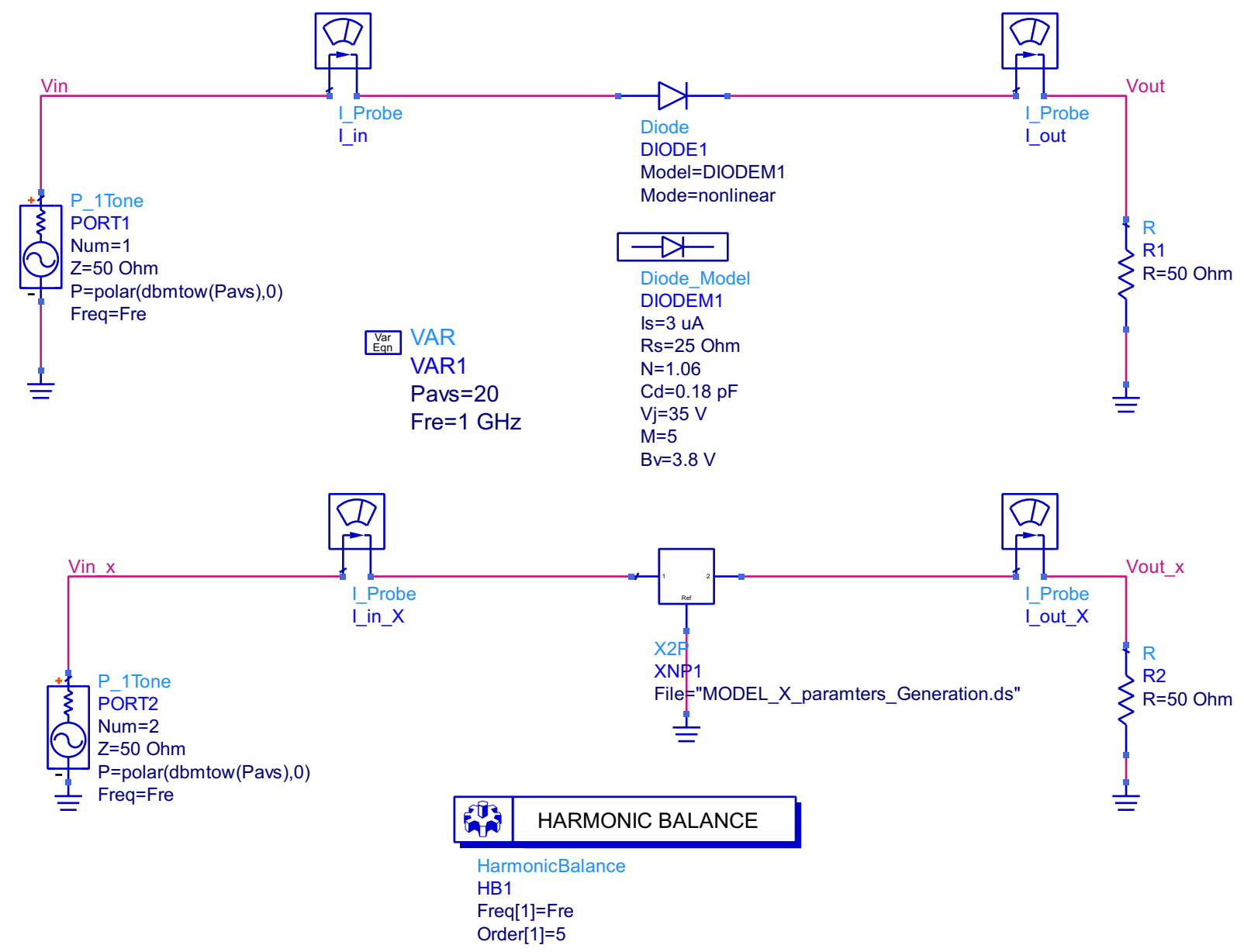

Figure 3.4: Comparison between circuit and X-Parameter model layout.

radiating patch can be rectangular, triangular, ring-shaped, circular, and so on. Antenna characteristics such as resonance frequency, polarization, input impedance, and radiation pattern are all influenced by the shape. Triangular Patch Antenna (TPA) was chosen for this study because it has a small footprint compared to other designs. The following formula can be used to compute the resonant frequency of an equilateral TPA with a side length of a [89, 108]:

$$
f_{m, n}=\frac{2 c}{3 a \sqrt{\epsilon_{r}}} \sqrt{m^{2}+m n+n^{2}}
$$




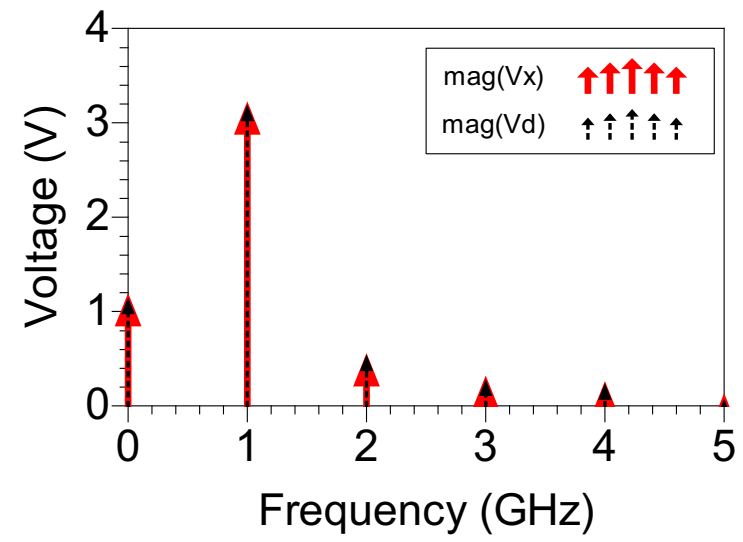

(a)

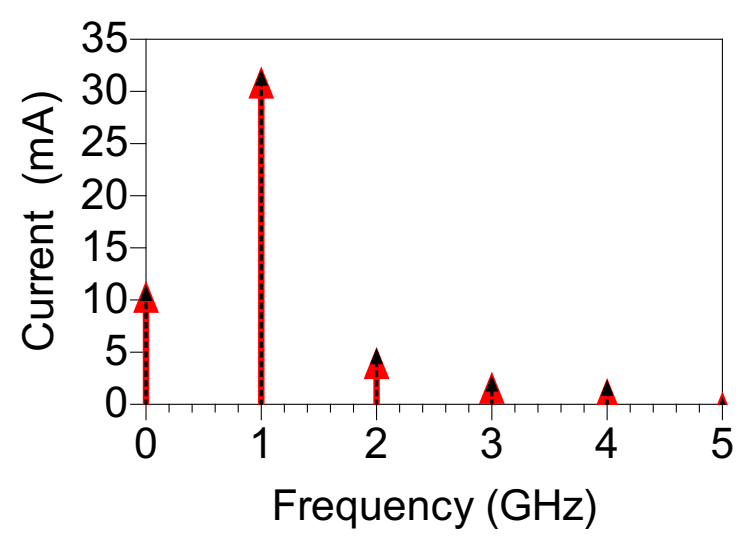

(b)

Figure 3.5: (a) Simulated voltage obtained from circuit model and X-Parameters model. (b) Simulated current obtained from circuit model and X-Parameters model.

where $\mathrm{c}$ is the velocity of light in free space, $m$ and $n$ are integers which are never zero simultaneously ( $f_{1,0}$ is the fundamental frequency), and $\epsilon_{r}$ is the dielectric constant of substrate.

Here I developed a TPA to operate over the 1.8 to $2.2 \mathrm{GHz}$ frequency range utilizing the optimization in CST mircowave studio simulator. Figure 3.6 shows the TPA with its dimensions and the angle reference for the incident field. As depicted in the Figure, the patch is based on an equilateral triangular design loaded with slots in the xy-plane. Due to embedding slots on the patch with a proper shape and size, two adjacent resonant modes merge together to form the bandwidth of the TPA. Moreover, introducing the slots reduces the large inductive reactance, consequently, impedance matching becomes easier over the frequency bandwidth.

The design of the TPA comprises a stack of three substrate layers sandwiched by the ground and the patch. The layers thicknesses from the top are; $1.27 \mathrm{~mm}, 11.71 \mathrm{~mm}$ and $1.52 \mathrm{~mm}$. Whereas the layers' dielectric constants from the top are 2, 1 and 3.66. The TPA was fed by a coaxial cable located at $26.4 \mathrm{~mm}$ from the base of the triangle as illustrated in Fig.3.6(a). Figure 3.6(d) shows the realized SMA connector.

Figure 3.7 shows the simulated and measured reflection coefficient (S11) of the TPA. Figure 3.7 shows good agreement between the simulated and measured reflection coefficients of the TPA. The simulated reflection coefficient was below $-10 \mathrm{~dB}$ within the frequency range 1.796 to $2.2 \mathrm{GHz}$, whereas the measurement results show reflection coefficient 


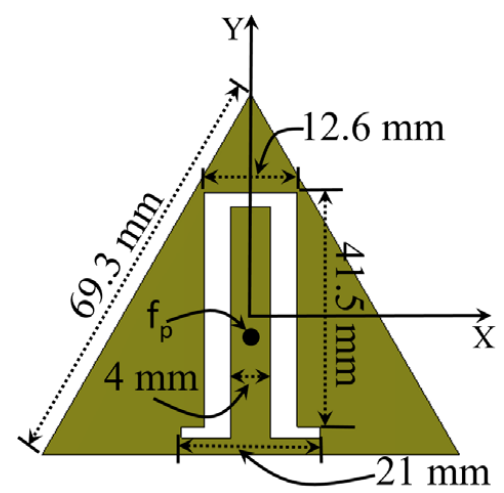

(a)

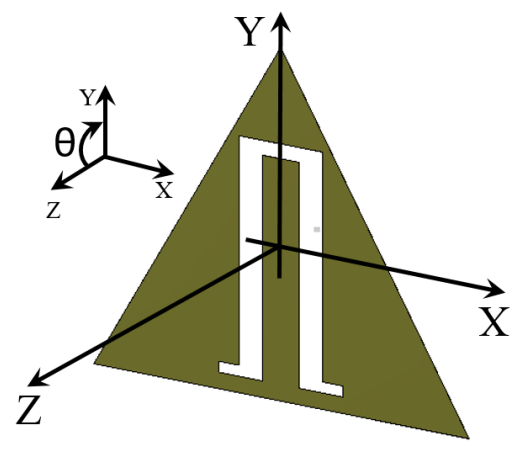

(c)

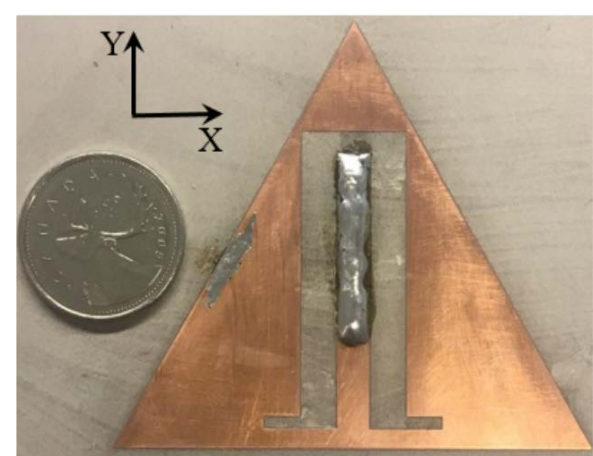

(b)

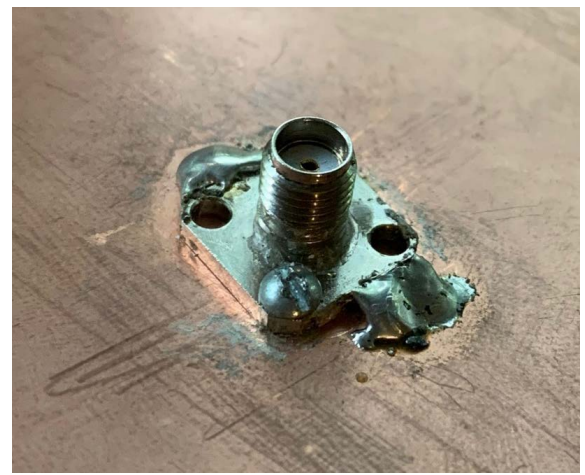

(d)

Figure 3.6: (a) Top view of the TPA with its dimensions. (b) The fabricated TPA. (c) Coordinate system used showing the angle $\theta$ of the incident field. (d) The fabricated feeding port of the TPA.

less than $-10 \mathrm{~dB}$ for the frequencies ranging from 1.75 to $2.26 \mathrm{GHz}$. For emphasis, we note that this antenna was chosen without loss of generality, and only for the purpose of the application of the method presented in this work.

\section{Designing a Matching Circuit Using S-parameters}

In Figure 3.8, $P_{\text {incident }}$ represents the total coming power from RF sources; $P_{\text {received }}$ is the power delivered to the diode; $P_{R A}, P_{R R}$, and $P_{R L}$ are the reflected power components at the front end of antenna, rectifying circuit, and the DC load, respectively; $P_{D C}$ is the output DC power. The rectenna-system's efficiency is defined as the ratio of the output DC power 


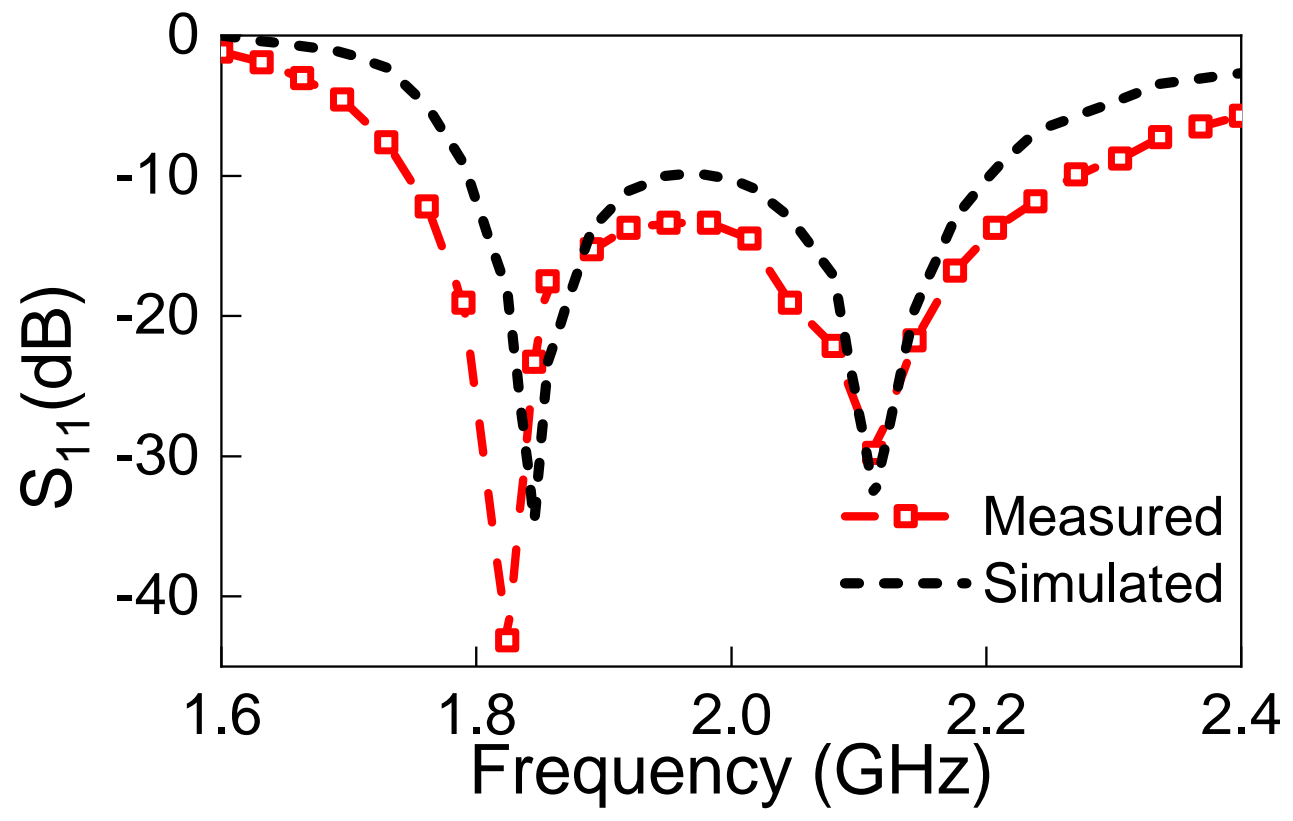

Figure 3.7: Simulated and measured reflection coefficient, $\mathrm{S}_{11}$, of the proposed TPA.

$\left(P_{D C}\right)$ to all coming power $\left(P_{\text {incident }}\right)$ :

$$
\eta=\frac{P_{D C}}{P_{\text {incident }}} \times 100 \%=\frac{V_{D C}^{2} / R_{L}}{P_{\text {incident }}} \times 100 \%
$$

To enhance the conversion efficiency of the rectifying circuit, the power delivered to the diode $\left(P_{\text {received }}\right)$ should be as much as possible. This can be achieved by designing an efficient matching circuit.

To design a matching circuit, the diode's S-parameters should be extracted. However, because of diodes' non-linearity, their S-parameters depend on the power level and the operating frequency. Then, to be able to extract the S-parameters of a nonlinear device, the power level and the operating frequency should be fixed. Utilizing a numerical simulator, Advance Design Simulator (ADS), I was able to design a matching circuit based on the extracted S-parameters yet at a very narrow band.

Figure 3.9 shows a schematic circuit of a diode (HSMS2860) connected to two ports using ADS. As show in the Figure, each port is $50 \Omega$ impedance. Figure 3.10 shows the variation of diode's reflection coefficient with the operating frequency at fix level of input power of $0 \mathrm{dBm}$. Figure 3.11 shows the variation of the reflection coefficient with the input power at frequency of $2 \mathrm{GHz}$. 


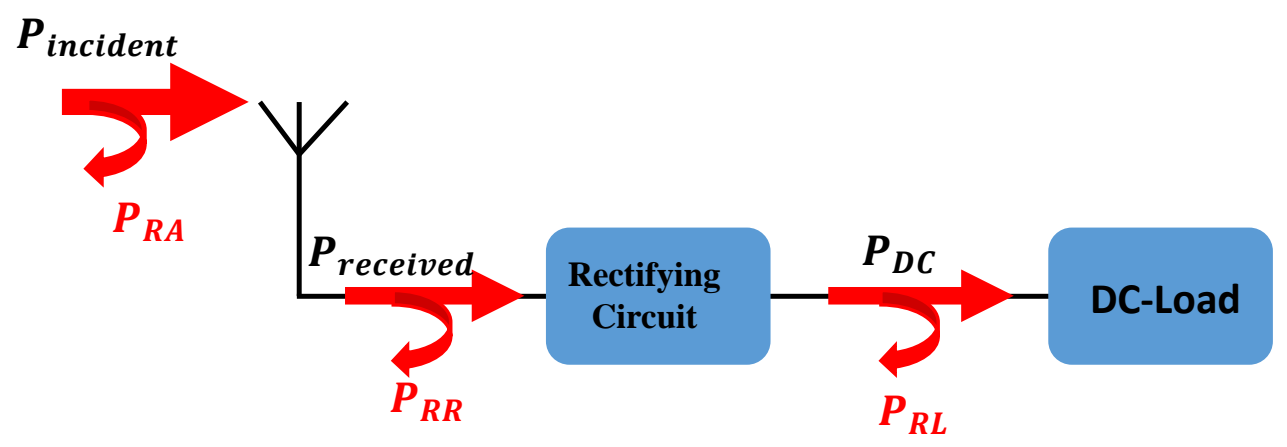

Figure 3.8: Power of rectifying circuit.

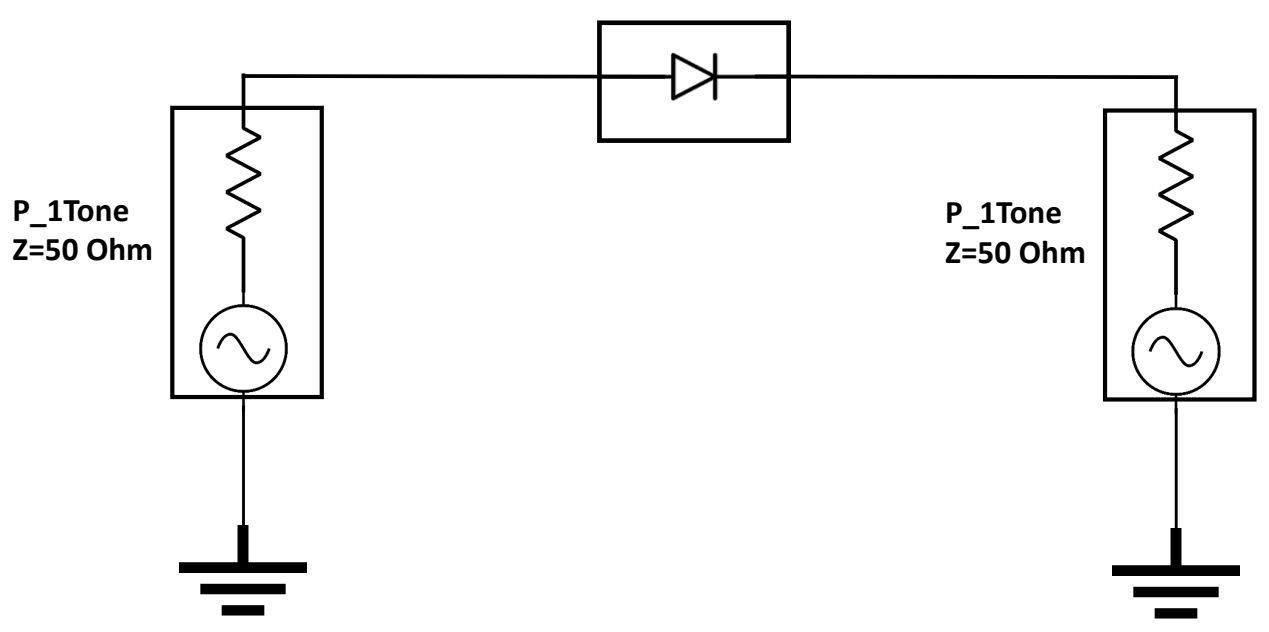

Figure 3.9: ADS diode circuit to extract S-parameters. 


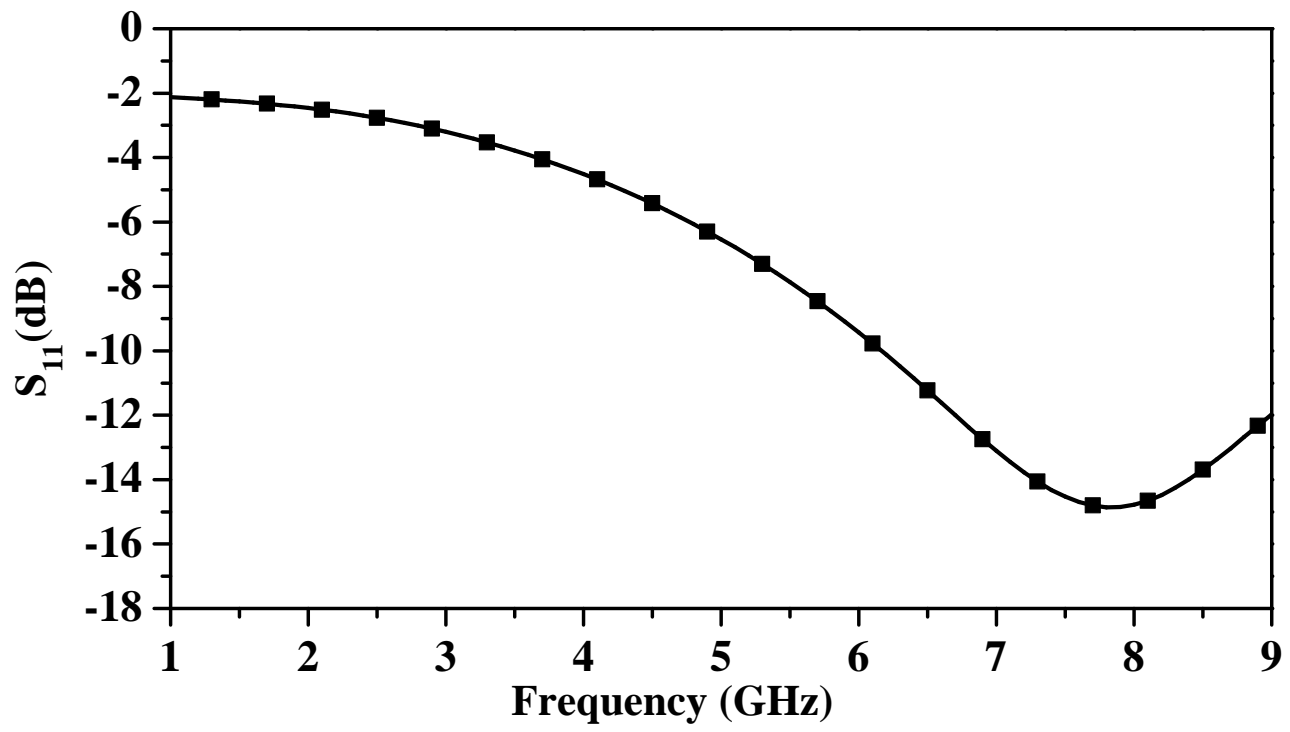

Figure 3.10: Variation of $S_{11}$ of the diode with frequency at input power of $0 \mathrm{dBm}$.

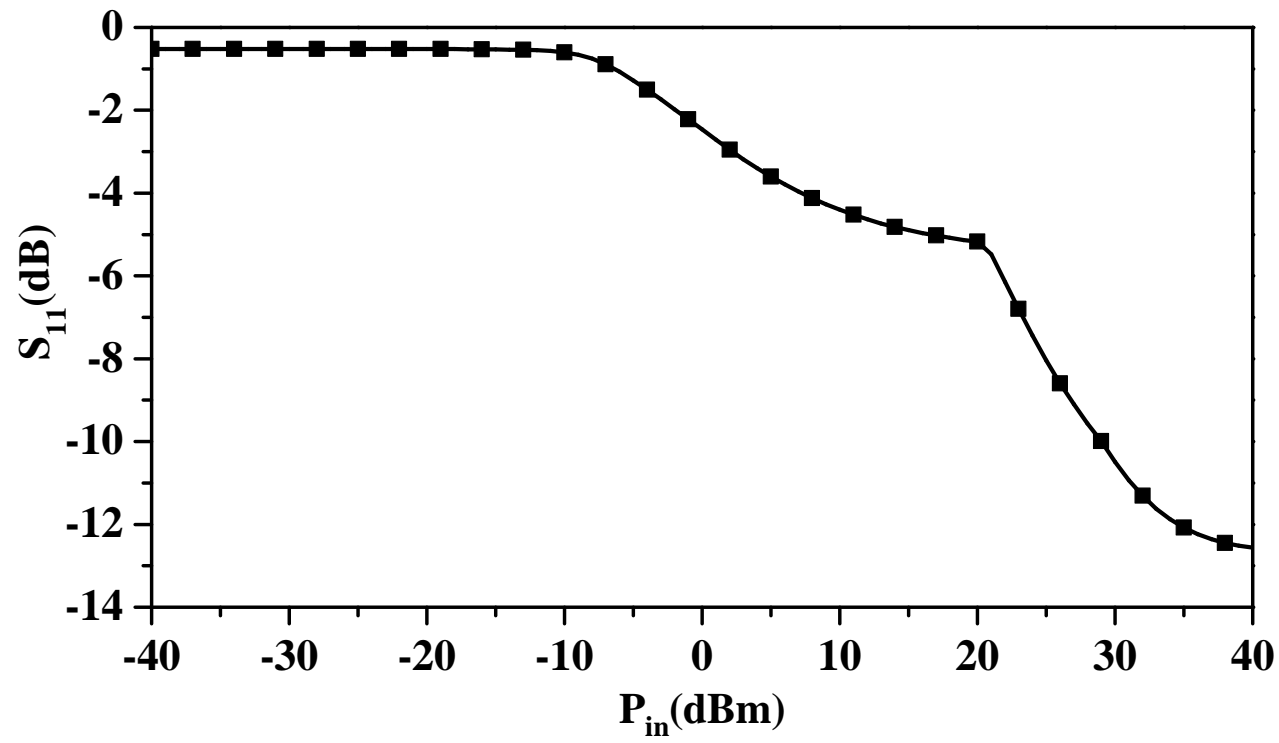

Figure 3.11: Variation of $S_{11}$ of the diode with input power at frequency of $2 \mathrm{GHz}$. 
After obtaining the required S-parameters, I used smith chart tool in ADS to design a matching circuit. Figure 3.12 illustrates the schematic circuit of the designed rectenna system. As illustrated in the Figure, it consists of 6 segments of transmission line applied on the top of a $1.52 \mathrm{~mm}$ thick RT/duroid 4003C Rogers dielectric substrate having a loss tangent of $\tan \delta=0.0027$ and a dielectric constant of $\epsilon_{r}=3.38$. The dimensions of these segments are shown in Table 3.1. The circuit is terminated by a parallel combination of $120 \mathrm{pF}$ capacitance and a load resistance of $1 \mathrm{~K} \Omega$. The designed rectifying circuit is designed and optimised to operate around $2 \mathrm{GHz}$.

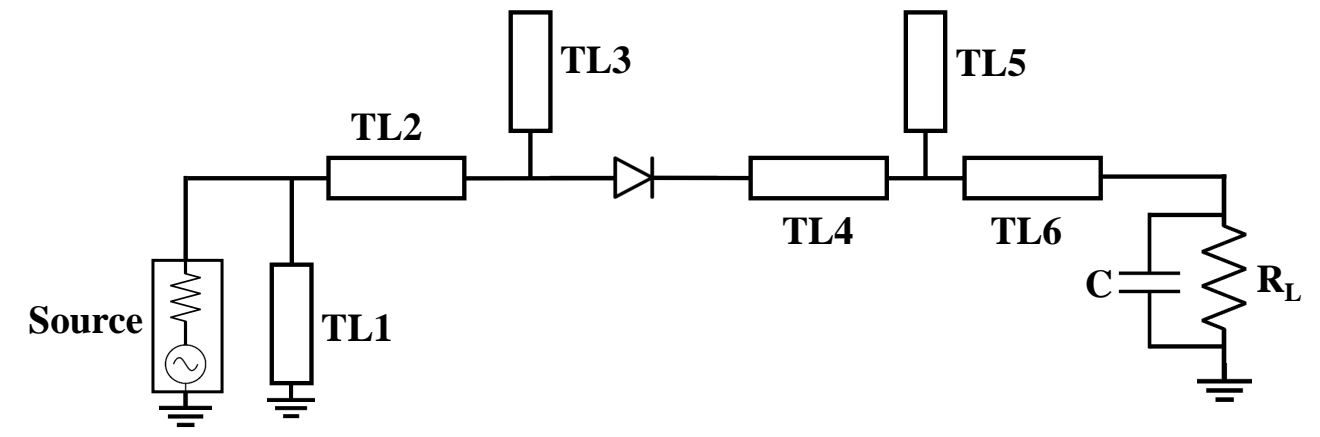

Figure 3.12: A schematic showing the rectifier circuit designed in ADS.

Table 3.1: Parameters of the transmission lines segments of the matching circuit .

\begin{tabular}{|c|c|c|}
\hline TLn & Length $\mathbf{( m m})$ & Width $\mathbf{~} \mathbf{m m})$ \\
\hline TL1 & 0.75 & 6.9 \\
\hline TL2 & 6.60 & 0.5 \\
\hline TL3 & 8.20 & 1.2 \\
\hline TL4 & 16.25 & 0.8 \\
\hline TL5 & 1.24 & 1.5 \\
\hline TL6 & 1.50 & 3.7 \\
\hline
\end{tabular}

To simulate a complete rectenna system using X-Parameters, I designed the rectenna system shown in Figure 3.12. Then, I extracted the X-Parameters of the whole circuit as shown in Figure 3.13. The X-Parameters are extracted at different values of power ranging from $-3 \mathrm{dBm}$ to $-1 \mathrm{dBm}$. Once the simulation of $\mathrm{X}$ parameters is finished, I open a new page of simulation in which I place the "Harmonic Balance" (HB) module as shown in Figure 3.14. 


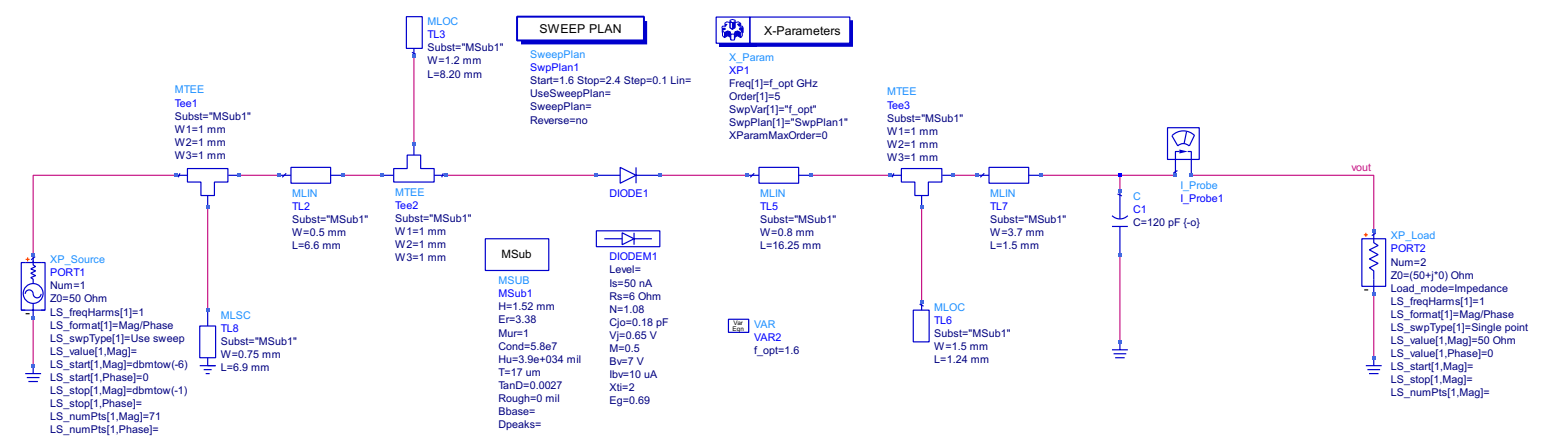

Figure 3.13: Generation of X parameters of a complete rectenna system.

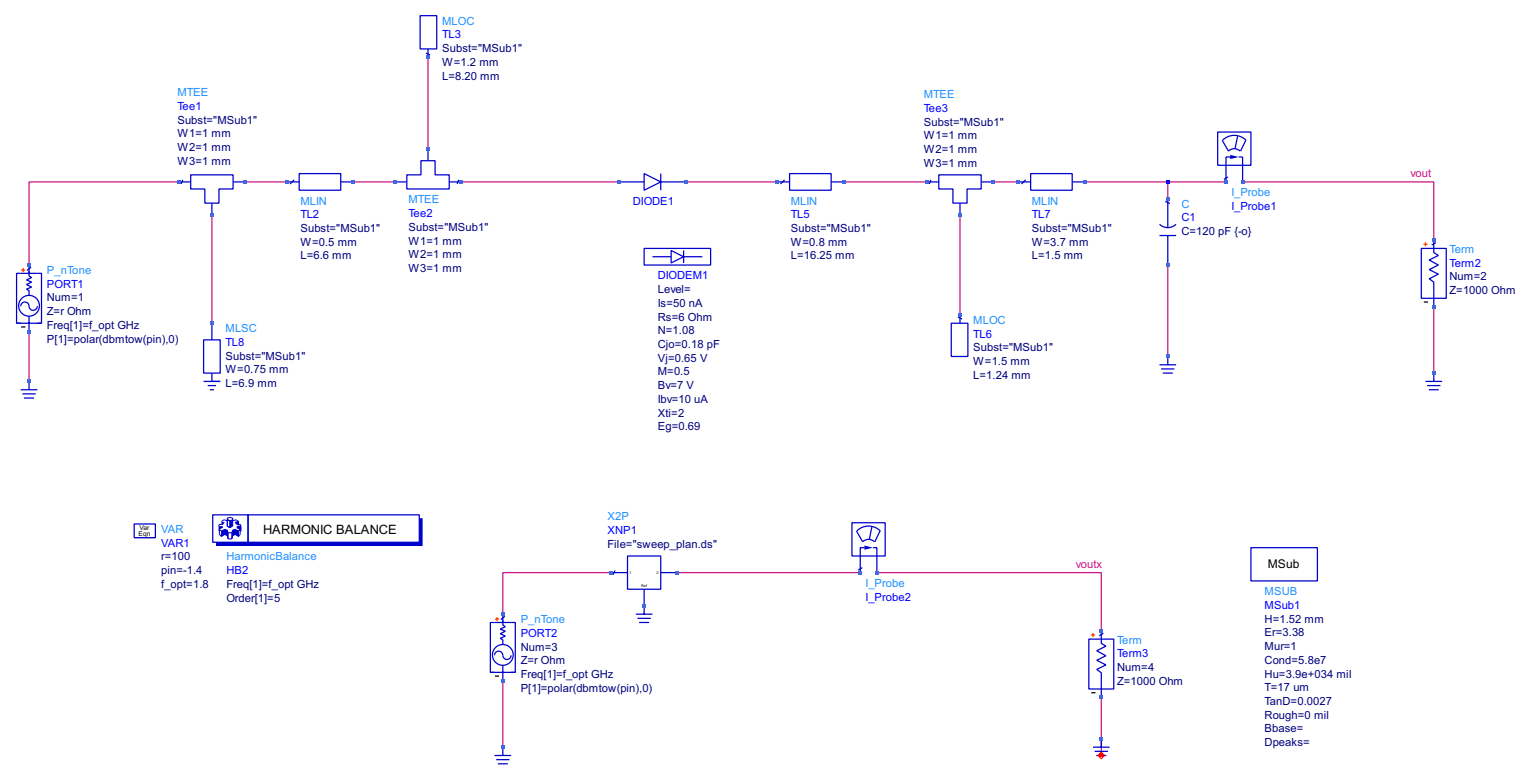

Figure 3.14: Comparison between circuit and X-Parameter model layout. 
In this simulation, I aim to compare between the results obtained by performing the harmonic balance simulation directly to the rectenna system and the results obtained by performing harmonic balance simulation to X-Parameter block. In this demonstration the frequency varies from 1.9 to $2.1 \mathrm{GHz}$, and the power varies from -3 to $-1 \mathrm{dBm}$.

Figure 3.15 shows the output voltage at fundamental frequency of $2 \mathrm{GHz}$ and at different harmonics. As shown in the Figure, the output DC voltage, using circuit simulation, is $0.615 \mathrm{~V}$ which is very close to the output $\mathrm{DC}$ voltage using $\mathrm{X}$-Parameters $(0.616 \mathrm{~V})$. The output voltages at the fundamental frequency and the harmonics are approximately 0 due to the capacitance load.

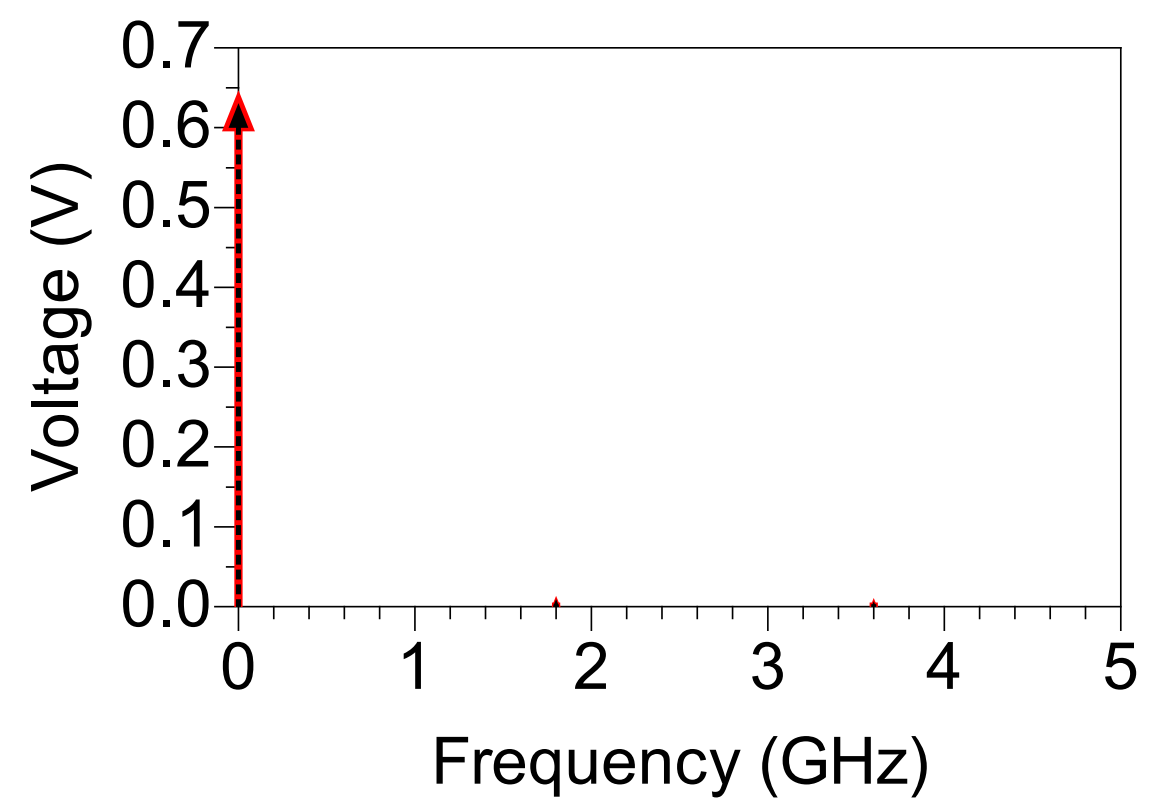

Figure 3.15: The output DC power at $2 \mathrm{GHz}$ in both circuit model and X-Parameters' model.

Table 3.2 shows the output DC voltages of both the circuit model $\left(V_{D C C}\right)$ and $\mathrm{X}$ Parameters box $\left(V_{D C X}\right)$ at different frequencies and their corresponding input power. The agreement between both results verifies that X-Parameters can replicate the behaviour of the whole circuit. 
Table 3.2: Output voltage of the rectenna system using both circuit model and XParameters.

\begin{tabular}{|c|c|c|c|c|}
\hline Freq(GHz) & $P_{\text {received }}$ & Antenna impedance & $V_{D C C}$ & $V_{D C X}$ \\
\hline 1.90 & -1.4 & $73.6+\mathrm{j} 19.1$ & 0.659 & 0.648 \\
\hline 1.95 & -1.7 & $95.6+\mathrm{j} 18.7$ & 0.654 & 0.639 \\
\hline 2.00 & -2.0 & $98.8+\mathrm{j} 2.2$ & 0.615 & 0.616 \\
\hline 2.05 & -2.2 & $81.2-\mathrm{j} 6.5$ & 0.593 & 0.596 \\
\hline 2.10 & -2.5 & $60.7+\mathrm{j} 0.7$ & 0.579 & 0.581 \\
\hline
\end{tabular}

\subsection{Conclusion}

In this chapter, I presented the concept of utilizing X-Parameter model to simulate the nonlinear devices. The X-Parameters can be extracted by measurements or by simulation. The process of extracting X-parameters utilizing ADS and utilizing them in simulation is fully explained. The results obtained utilizing circuit model of a diode is compared with results obtained utilizing X-Parameters. The comparison results show the applicability of $\mathrm{X}$-Parameters and their ability to mimic the behaviour of a nonlinear device.

To simulate a rectenna system utilizing X-Parameters, I divided the system into two parts; electromagnetic part, and circuit part. The electromagnetic part consists of source, transmitting antenna, and receiving antenna. The electromagnetic part was replaced by an equivalent circuit consists of power source and impedance. On the other hand, the circuit part consists of matching circuit, diode, and load. The X-Parameters of the circuit part were extracted and utilized in simulation. A comparison between X-Parameters model and circuit model was conducted. The good agreement between both method shows that the $\mathrm{X}$-Parameters can replace the circuit model which take relatively long time to develop. 


\section{Chapter 4}

\section{Simulation and Optimization of Rectenna}

\subsection{Introduction}

Driven by unprecedented demands on energy, the need for new paradigms that produce clean and affordable energy becomes increasingly desired. Recently, there has been intensive research to investigate the ability of rectenna systems to harvest the energy from electromagnetic (EM) radiation (for a sample of recent works, please see [38, 39, 40, 41, $42,43,44,45,46,47,48,49,50,51,52]$ and references therein). To harvest EM radiation, a rectenna is used, which consists of an antenna or a surface that captures the EM radiation, a rectifying circuit, and a DC load circuit. The antenna acts as a transducer that converts the incoming EM radiation into an electrical signal. The rectifying circuit, which consists of a single or multiple diodes and a matching circuit, converts the AC power into DC power.

The rectenna system can be used in a power transmission link (point-to-point), where the design constraints of the rectenna can be tailored to the nature of the largely known (or predictable) incident field, including its frequency, polarization and power density (see [74] as an example), or can be used to harvest ambient EM radiation over specific frequency band (see [75, 76] as an example). In the latter application, where the rectenna acts as an energy scavenger, since the incoming radiation is highly unpredictable in terms of frequency bandwidth, polarization and power density, the design of the rectenna can be challenging.

Harvesting the ambient EM radiation was investigated in urban and semi-urban areas. For instance, in [75], it was demonstrated that an ambient EM wave with power density 
of $-25 \mathrm{dBm} / \mathrm{cm}^{2}$ can be harvested with $40 \%$ efficiency using a single band rectenna, and an ambient wave with power density of $-29 \mathrm{dBm} / \mathrm{cm}^{2}$ can be harvested with a multi-band rectenna system. To evaluate the efficiency of a system with fluctuating levels of available power, in [75], the efficiency was calculated using energy instead of power.

One of the key challenges when designing a rectenna system is the non-linearity of the rectifying circuit. Due to the diode's non-linearity, the input impedance of the rectifying circuit is sensitive to the operating frequency and power level. Due to the non-uniform input impedance of antennas with frequency, any change in the operating frequency or in the angle of incidence would result in a change in the available power at the terminals of the antenna, which feed the rectification circuit. Consequently, to properly simulate the behaviour of the complete rectenna, all variations in the power level, the input impedance, and the frequency should be taken into consideration. This challenge is demonstrated by numerous previous works. For example, Lu et al. proposed a configurable rectenna system that operates at two different frequencies where the efficiency of the system was evaluated for different values of power; however, an agreement between the simulation and measurement results was obtained only at a specific power level [109]. Nie et al. obtained good agreement between the simulation results and measurements only over a narrow-band close to the operating frequency; however, outside this narrow-band, the simulation results deviated from the measurement results significantly [110]. In other works, the variation of the input impedance of the antenna with frequency was taken into account, however, the input power was assumed constant with with frequency [17, 80, 16]. In these works the simulation procedure gives accurate prediction of the rectenna performance over a very narrow frequency range; however, for other frequencies, the prediction would deviate appreciably from the actual performance. In [111], the Co-simulation was utilized to import the S-parameters of an antenna from an EM-based simulator to a circuit-based simulator, where the S1P file was utilized to incorporate the characteristic of the antenna's input impedance versus frequency in the rectifying circuit's input port. The fluctuation of the antenna's input impedance with frequency was taken into consideration, but still the input power was supposed to be constant.

To achieve high rectenna efficiency, Selim et al. divided the input power levels into two ranges: a low range $(-50$ to $0 \mathrm{dBm})$, and a high range (0 to $30 \mathrm{dBm})$ [112]. Then two circuits were designed: one for the high range, and a second one for the low range. The two circuits were then combined to give the final rectenna system. While Selim et al. claimed that the input voltage depends on the input power, no explanation of the relationship between the input voltage and the received power was provided. Moreover, the effect of the variation of the voltage with frequency was not analysed.

In this work, I present a method by which the entire rectenna system can be fully 
simulated while taking the variation of the incident power density, the input impedance of the antenna, the impedance of the rectification circuit, and frequency into account. Specifically, the simulation presented would allow for the output DC power to be accurately predicted for any level of available electric field intensity at the antenna and over a range of frequencies.

The rest of the paper is ordered as follows: section two presents the theory underlying the present method. Section II presents the theory behind the our method. Section III demonstrate the application of the method to a rectenna employing a triangular patch antenna. Section IV gives a full validation of the method using measurements. Section V provides a summary of the main contributions.

\subsection{Theory}

Figure 4.1 shows diagram of the basic components of a generic complete rectenna system. As depicted in the Figure, the rectenna includes the antenna that receives the incoming EM radiation, and the circuit part comprising a rectifying circuit and a DC load. In Fig. 4.1, $P_{\text {inc }}$ represents the incoming power density at the antenna from an external electromagnetic source, $P_{\text {rec }}$ is the power received at the terminals of the antenna, and $P_{d c}$ is the output $\mathrm{DC}$ power. There are two efficiencies that combine to give the full rectenna efficiency. The first is the radiation to $\mathrm{AC}$ conversion efficiency defined as the ratio between $P_{\text {rec }}$ and $P_{\text {inc }}$ :

$$
\eta_{\text {rad }-a c}=\frac{P_{r e c}}{P_{\text {inc }}}
$$

where $P_{\text {inc }}$ is the power available at the antenna, and $P_{r e c}$ is the power available the terminals of the antenna (all are time-average power). The second is the AC to DC conversion efficiency, which is defined as

$$
\eta_{a c-d c}=\frac{P_{d c}}{P_{r e c}}=\frac{V_{d c}^{2} / R_{L}}{P_{r e c}}
$$

where $R_{L}$ is the load resistance, and $V_{d c}$ is the voltage across $R_{L}$. The full rectenna efficiency is then given by

$$
\eta=\eta_{r a d-a c} \times \eta_{a c-d c}
$$

To maximize power transfer from the antenna terminals to the input of the diode, a matching circuit is placed between the antenna and the diode or the rectification circuit (such matching circuit may be considered as part of the rectification circuit). Due to the 


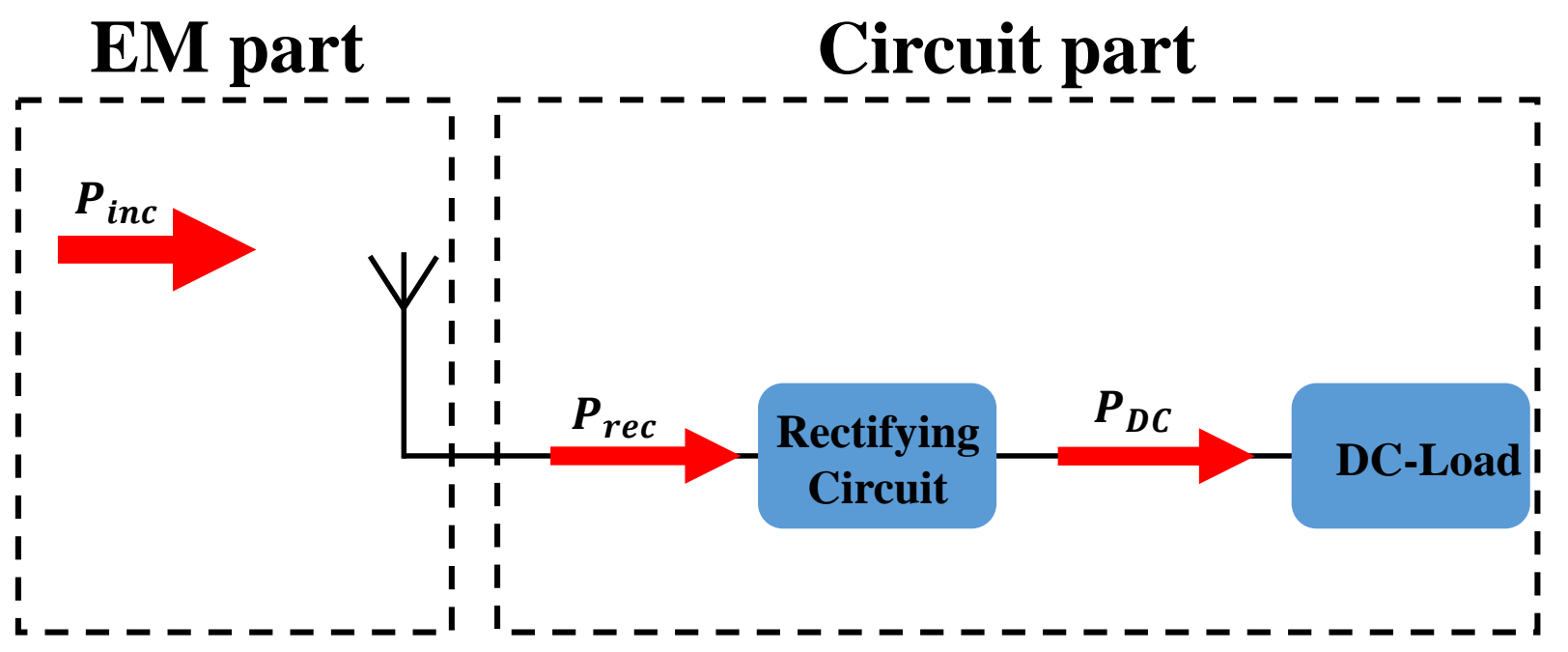

Figure 4.1: Basic components of a generic rectenna system comprising a receiving antenna, a rectification circuit (may include matching circuits on both ends) and a DC load.

non-linearity of the rectification circuit, its input impedance is sensitive to variations of the input power. This variation is a major challenge that prevented full characterization of the rectenna system over the operating frequency range of the rectenna. To demonstrate the sensitivity of the rectification circuit's input impedance to the input power, I simulated the reflection coefficient of an HSMS-2860 diode (chosen as as an example of the rectification circuit) for different levels of power, using the Advance Design Simulator (ADS) software [104]. Figure 4.2 shows the reflection coefficient of the diode for different values of power at $2 \mathrm{GHz}$ when the diode is terminated by a $50 \mathrm{Ohm}$ load. I observe that the reflection coefficient decreases with increasing input power. The wide variation of the reflection coefficient with the input power demonstrates the need to incorporate the power profile at the antenna terminals into the simulation of the entire rectenna system. If the power profile was not taken into account, the variation of the power level at the input of the rectification circuit would result in a significant discrepancy between the simulation's results and the measurement's results as has been demonstrated in previous works [17, 80, 16].

The intuitive brute-force approach to incorporate all rectenna components into the simulation process entails combining the full-wave 3D model of the antenna and the circuit models of the rectification circuit (its linear and non-linear parts) in such a manner that the frequency bandwidth of the simulation stretches from the operating frequency of the antenna to DC while including all harmonics arising from the non-linear rectification circuit. Such approach may not be impossible but would be highly challenging and most 


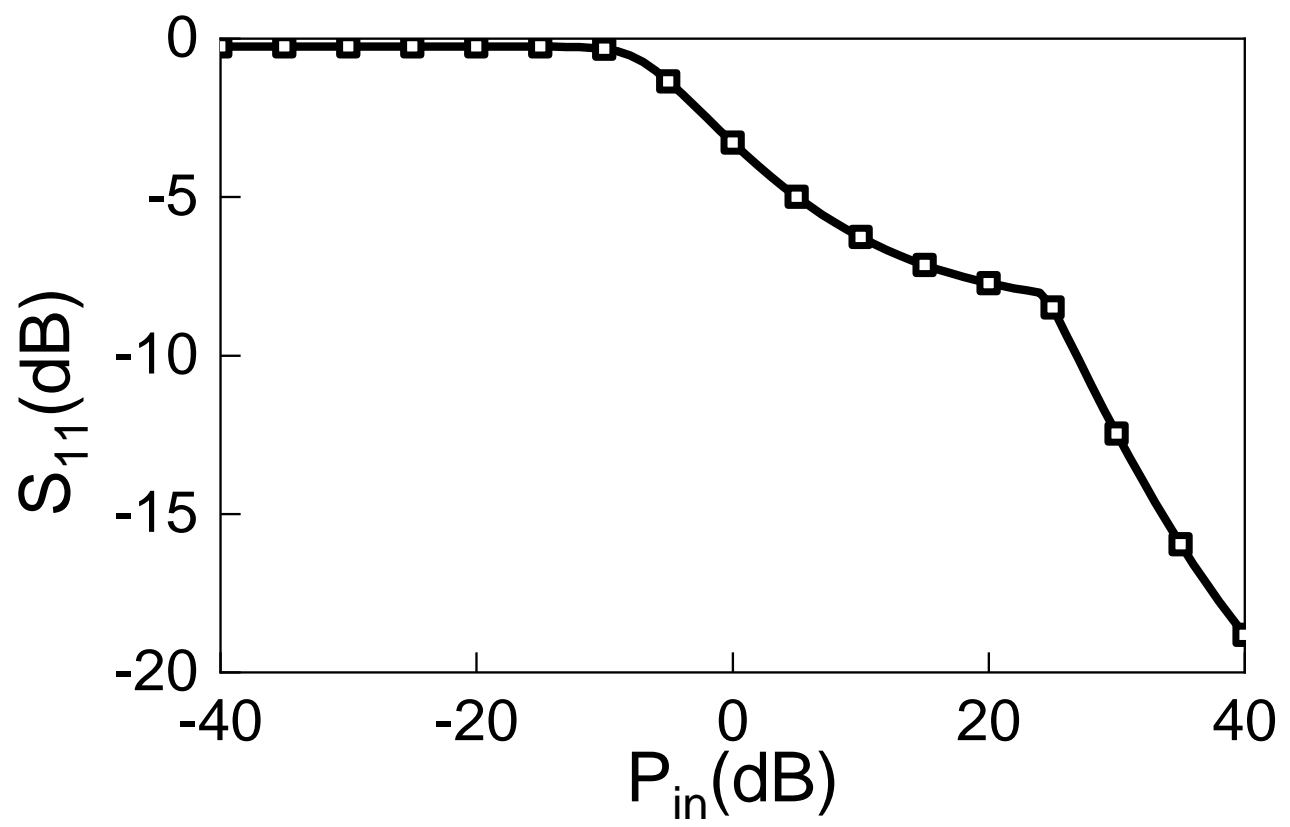

Figure 4.2: Variation of reflection coefficient of the diode with input power at $2 \mathrm{GHz}$.

likely very computationally intensive. Another possibility would be to incorporate the VI characteristics of the non-linear circuit into a full-wave time-domain simulator such as the finite-difference time-domain (FDTD) method (see [113] as an example where diodes were incorporated into the FDTD method). Such approach have significant challenges and limitations, which may require lumping the entire circuit (the linear and non-linear components) as a single load terminating the antenna (while occupying a single FDTD cell). Even if workable, such approach may be suited for simulating electromagnetic scattering problems rather simulating rectenna systems, particularly because the DC power at a specific load is desired, which preclude lumping all circuits into one element having a specific I-V characteristics. Additionally, the three-dimensional full-wave model may not be suitable for optimization of rectification circuits to achieve maximum power transfer to the load.

Nevertheless, while time-domain simulation methods may be the most suitable "bruteforce" method to simulate the performance of the entire rectenna system, the convergence of time-domain methods depends strongly on the time step, which can significantly increase the computational cost. Here I need to emphasize that the modeling approach needs to keep in mind an important objective when simulating rectenna systems, which is maximizing energy transfer from the antenna to a DC load. Therefore, a highly useful simulation 
strategy should allow for optimization of the matching and rectification circuits to achieve highest output DC power.

Instead of using a brute-force approach, our simulation methodology calls for modeling the antenna part as a circuit that is fully independent in its characteristics from the remaining parts of the rectenna system. This can be accomplished by using Thevenin theory. According to Thevenin theorem, a linear circuit can be replaced with an equivalent circuit consisting of an independent voltage source in series with an impedance as shown in Fig. 4.3(a). The Thevenin equivalent circuit provide all the required information about the antenna. It is important to emphasize that the equivalent circuit of the antenna obtained using Thevenin theory is not affected by the rectenna circuit that connects the antenna to the load. In Fig.4.3(a), $V_{\text {oc }}$ represents the open-circuit voltage generated between the receiving antenna's terminals when the terminals are left open and disconnected from the rectenna circuit. $Z_{\text {ant }}$ is the internal impedance of the receiving antenna.

While the antenna acts as an independent source as far as the rectenna circuit is concerned, if the source of radiation (such as a transmitter antenna) were included in the simulation of the antenna, the antenna would then be considered a dependent source. However, the goal of our simulation is to provide a prediction of the DC power for a range of incident power density and incident field polarization profiles. This would then provides, at the design stage, full prediction of the DC power for an incident field with specific power density and polarization. Therefore, $V_{o c}$ of the Thevenin equivalent circuit would correspond to an incident field with a specific power density and polarization.

To extract $V_{o c}$, I illuminated the antenna by a plane wave of fixed power density and fixed incident angle and polarization. The CST Microwave Studio simulator was used to simulate the antenna [114]. While $V_{o c}$ is dependent on the characteristics of the incident field, $Z_{a n t}$, however, is independent of these characteristics, and can be found by either feeding the antenna with a source and then taking the ratio of the feed voltage to the input current, or by calculating the current in a short circuit $I_{s c}$ and then taking the ratio $V_{o c} / I_{s c}$ which will be equal to $Z_{\text {ant }}$. While it may not be intuitive, the ratio $V_{o c} / I_{s c}$ will always be equal to $Z_{\text {ant }}$ irrespective of the characteristics of the incident field.

The extracted values of $V_{o c}$ are stored in data access component (DAC) in ADS. DAC acts as accessible database that stores a table of data and allows the simulator to recall the required value corresponds to the index value. Using the voltage source connected with $\mathrm{DAC}$ enables the voltage source to mimic the variation of the voltage across the terminals of antenna.

To simulate the variation of $Z_{\text {ant }}$ with frequency, I designed the equivalent circuit illustrated in Fig.4.3(b). The equivalent circuit comprises an inductor and a capacitor 


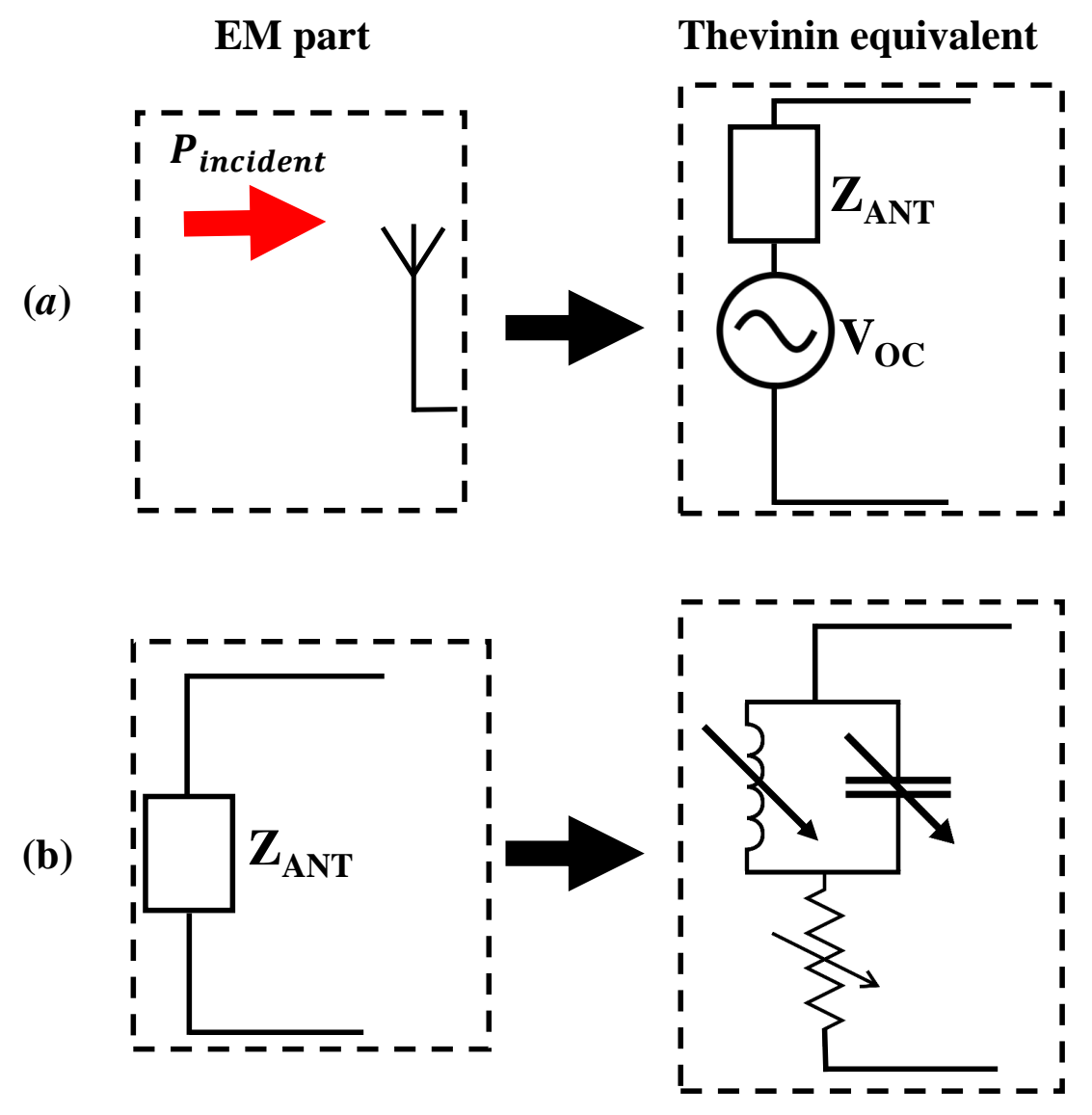

Figure 4.3: Equivalent circuit. (a) Thevenin equivalent circuit of an antenna. (b) Representation of the input impedance of the antenna.

connected in parallel and connected in series with a resistor. The values of real part of $Z_{\text {ant }}$ are assigned to the resistor (from the DAC data). The values of imaginary part of $Z_{\text {ant }}$ are used to calculate the corresponding value of capacitor or inductor. 


\subsection{Application}

\subsubsection{Design of the Antenna}

In this section, I demonstrate the applicability of our method to a rectenna system employing a practical antenna. As a receiving side, I utilized a Microstrip Patch Antenna (MPA), which consists of a metallic radiating patch printed on a dielectric substrate and backed by a ground plane. The radiating patch can be rectangular, triangular, ring-shaped, circular, and so on. Antenna characteristics such as resonance frequency, polarization, input impedance, and radiation pattern are all influenced by the shape. Triangular Patch Antenna (TPA) was chosen for this study because it has a small footprint compared to other designs. The following formula can be used to compute the resonant frequency of an equilateral TPA with a side length of a [89, 108]:

$$
f_{m, n}=\frac{2 c}{3 a \sqrt{\epsilon_{r}}} \sqrt{m^{2}+m n+n^{2}}
$$

where $\mathrm{c}$ is the velocity of light in free space, $m$ and $n$ are integers which are never zero simultaneously ( $f_{1,0}$ is the fundamental frequency), and $\epsilon_{r}$ is the dielectric constant of substrate.

Here I developed a TPA to operate over the 1.8 to $2.2 \mathrm{GHz}$ frequency range utilizing the optimization in CST mircowave studio simulator. Figure 4.4 shows the TPA with its dimensions and the angle reference for the incident field. As depicted in the Figure, the patch is based on an equilateral triangular design loaded with slots in the xy-plane. Due to embedding slots on the patch with a proper shape and size, two adjacent resonant modes merge together to form the bandwidth of the TPA. Moreover, introducing the slots reduces the large inductive reactance, consequently, impedance matching becomes easier over the frequency bandwidth.

The design of the TPA comprises a stack of three substrate layers sandwiched by the ground and the patch. The layers thicknesses from the top are; $1.27 \mathrm{~mm}, 11.71 \mathrm{~mm}$ and $1.52 \mathrm{~mm}$. Whereas the layers' dielectric constants from the top are 2, 1 and 3.66. The TPA was fed by a coaxial cable located at $26.4 \mathrm{~mm}$ from the base of the triangle as illustrated in Fig.4.4(a). Figure 4.4(d) shows the realized SMA connector.

Figure 4.5 shows the simulated and measured reflection coefficient (S11) of the TPA. Figure 4.5 shows good agreement between the simulated and measured reflection coefficients of the TPA. The simulated reflection coefficient was below $-10 \mathrm{~dB}$ within the frequency range 1.796 to $2.2 \mathrm{GHz}$, whereas the measurement results show reflection coefficient 


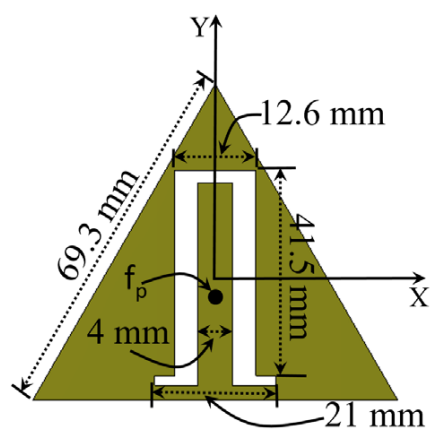

(a)

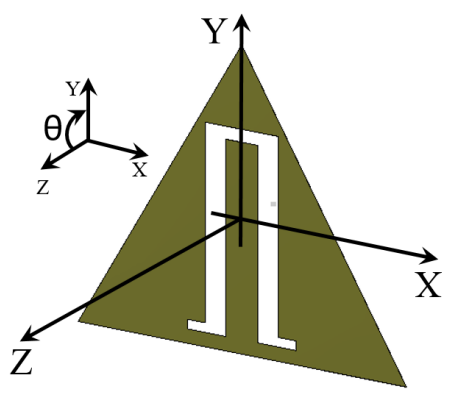

(c)

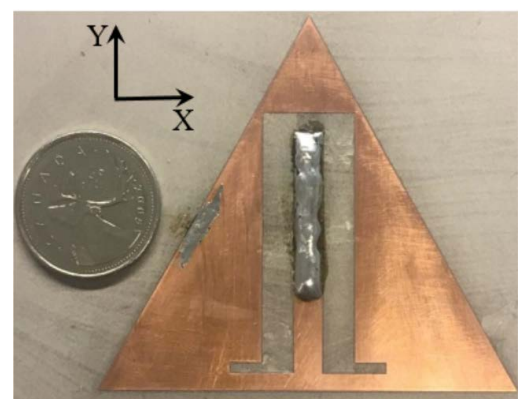

(b)

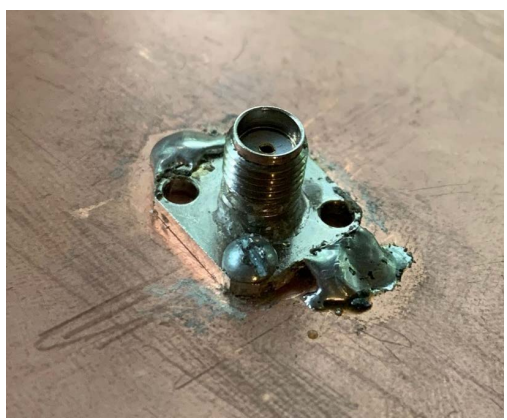

(d)

Figure 4.4: (a) Top view of the TPA with its dimensions. (b) The fabricated TPA. (c) Coordinate system used showing the angle $\theta$ of the incident field. (d) The fabricated feeding port of the TPA.

less than $-10 \mathrm{~dB}$ for the frequencies ranging from 1.75 to $2.26 \mathrm{GHz}$. For emphasis, we note that this antenna was chosen without loss of generality, and only for the purpose of the application of the method presented in this work.

\subsubsection{Design of Rectifying Circuit}

To design the rectifying circuit, I connected the Thevenin equivalent circuit of the TPA with the circuit shown in Figure 4.6. In ADS, I utilized the DAC component to control the values of each component in the equivalent circuit. As illustrated in the Figure, the circuit consists of 11 segments of transmission lines printed on the top of a $1.27 \mathrm{~mm}$ thick Roger 3006 dielectric substrate having a loss tangent of $\tan \delta=0.0027$ and a dielectric constant of $\epsilon_{r}=6.15$. To achieve maximum output DC power, the widths of transmission lines' segments were fixed to $1.8 \mathrm{~mm}$. The lengths of segments and the DC load were 


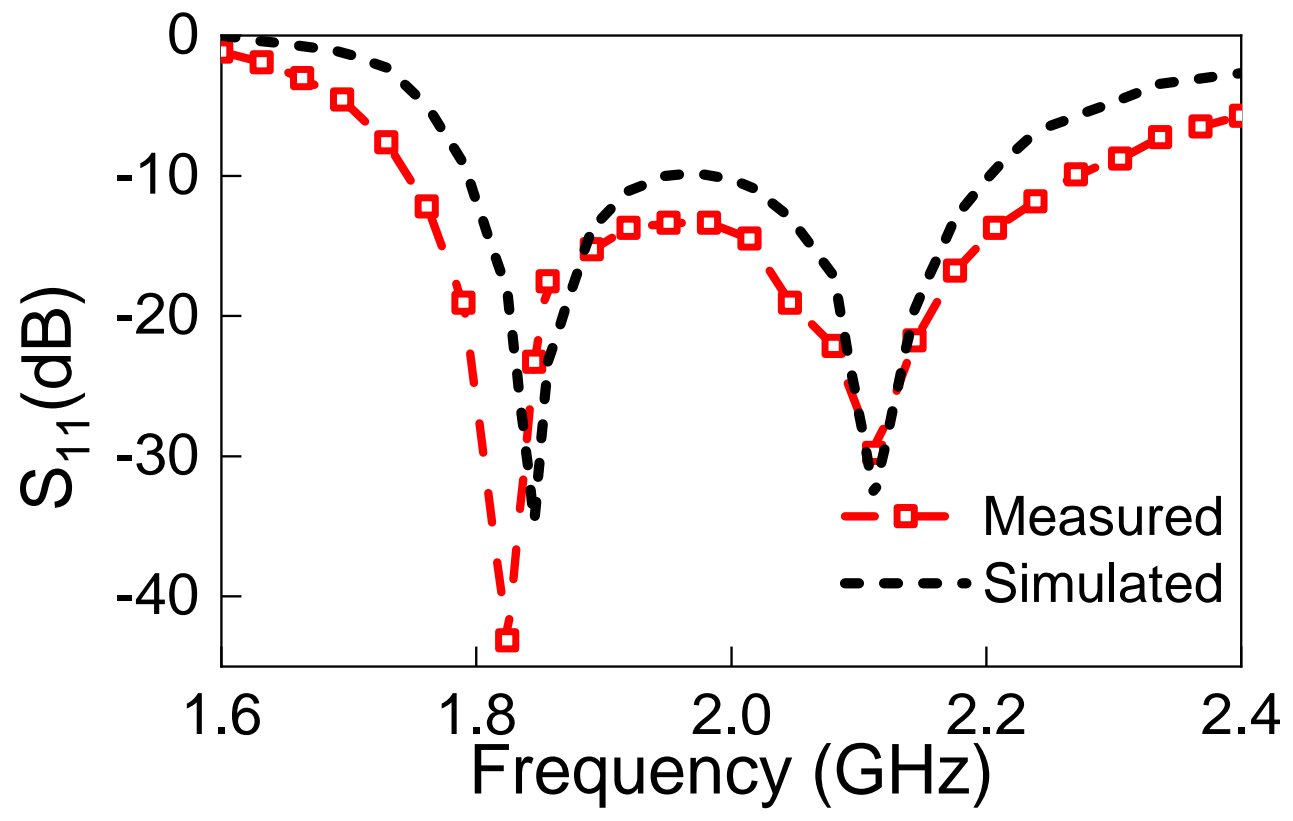

Figure 4.5: Simulated and measured reflection coefficient, $\mathrm{S}_{11}$, of the proposed TPA.

optimized using the gradient optimization feature in ADS. The lengths of the segments of the optimized circuit are shown in Table 4.1. The optimum load was found to be a parallel combination of $100 \mathrm{pF}$ capacitance and a resistance of $700 \mathrm{Ohm}$. It is important to note that the optimized circuit was based on an incident field with specific characteristics in terms of polarization, incident angle and power density. For wireless power transfer applications where the incident field characteristics are known, the optimized rectification circuit just described will deliver maximum power to the optimized load. However, for energy harvesting of incoming radiation without any known characteristics (aside from the frequency range), then the field-specific optimized rectification circuit may not be optimal over the possible variance in the characteristics of the incident field. However, I emphasize that the primary objective of this work is to enable full characterization (simulation) of the rectenna system irrespective of the type of rectification circuit used and without any fabrication of the circuit and the antenna.

Using the time-domain solver in CST, the antenna was illuminated by a linearlypolarized plane wave (y-polarization; see Fig. 4.4). The power density of the plane wave was $1 \mathrm{~W} / \mathrm{m}^{2}$ and the angle of incidence $(\theta)$ was zero degree (i.e., the $\mathrm{k}$ vector is coming from the positive z-direction. Then, $V_{o c}$ was extracted from the full-wave simulation of the TPA (when the antenna's terminals were open-circuited), and is shown in Fig. 4.7. Figure 


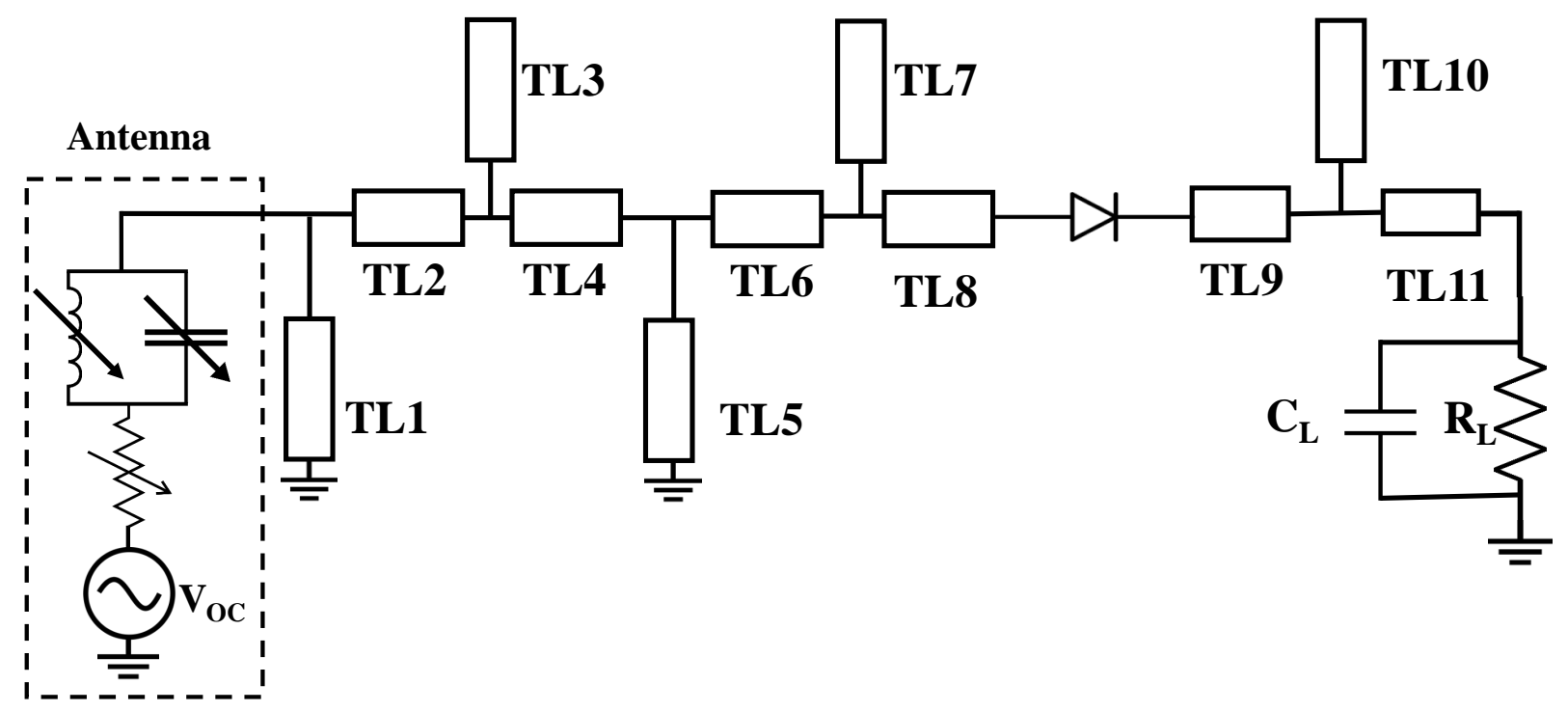

Figure 4.6: Schematic diagram of the rectifying circuit with the incorporated equivalent circuit of the antenna.

4.8 shows the simulated real and imaginary part of the TPA's impedance.

The extracted parameters are processed and loaded to the source as explained in the Theory section. Using ADS, the received AC power, $P_{r e c}$, and the output DC power, $P_{d c}$, of the rectenna system were obtained (ref to Fig.4.6). Figure 4.9 shows the simulation results of $P_{r e c}$ and $P_{d c}$. Figure 4.10 shows the efficiency of the rectifying circuit. The efficiency of the rectifying circuit is greater than $65 \%$ over the operating range, with a maximum of $70 \%$ at $1.92 \mathrm{GHz}$. 
Table 4.1:

Lengths of the transmission lines segments used in the optimized rectification circuit.

\begin{tabular}{|c|c|c|c|}
\hline TLn & Length $\mathbf{( m m )}$ & TLn & Length $\mathbf{( m m )}$ \\
\hline \hline TL1 & 14 & TL7 & 2 \\
\hline TL2 & 5 & TL8 & 2 \\
\hline TL3 & 6.6 & TL9 & 2.2 \\
\hline TL4 & 6.2 & TL10 & 2.2 \\
\hline TL5 & 5 & TL11 & 10 \\
\hline TL6 & 9 & & \\
\hline
\end{tabular}

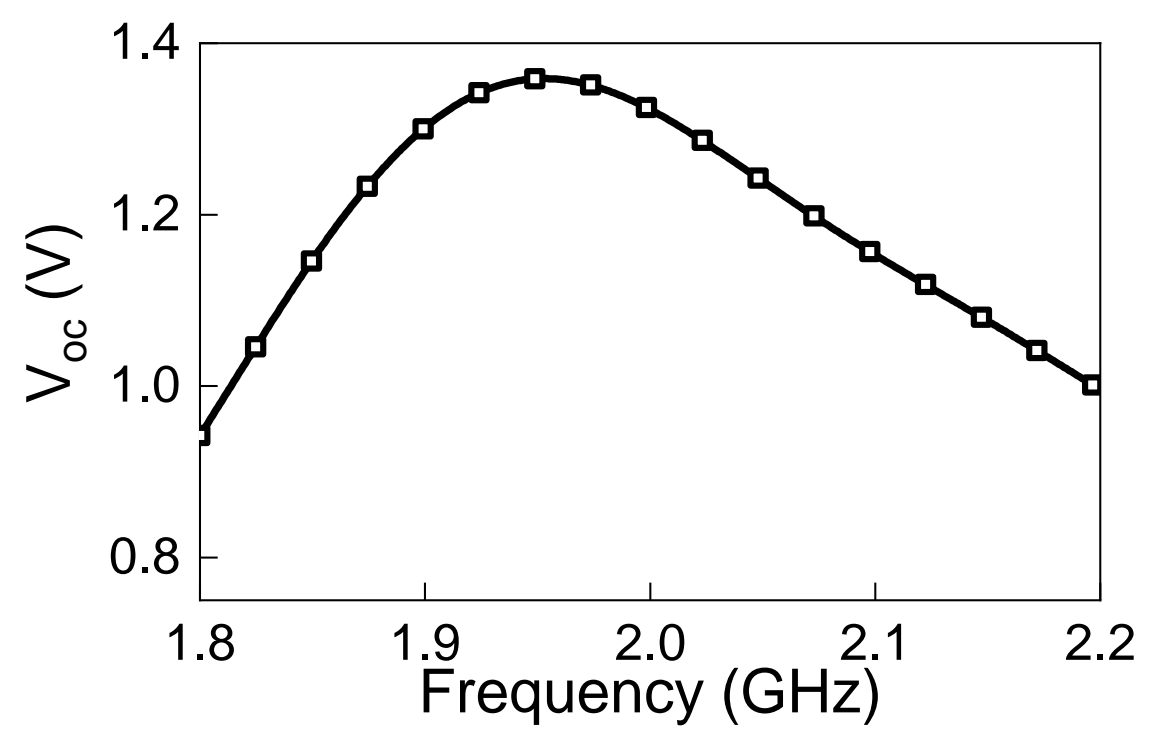

Figure 4.7: Simulated $V_{o c}$ for an incident plane wave having power density of $1 \mathrm{~W} / \mathrm{m}^{2}$, polarized in the y-direction and incident at an angle $\theta_{i n}=0$. 


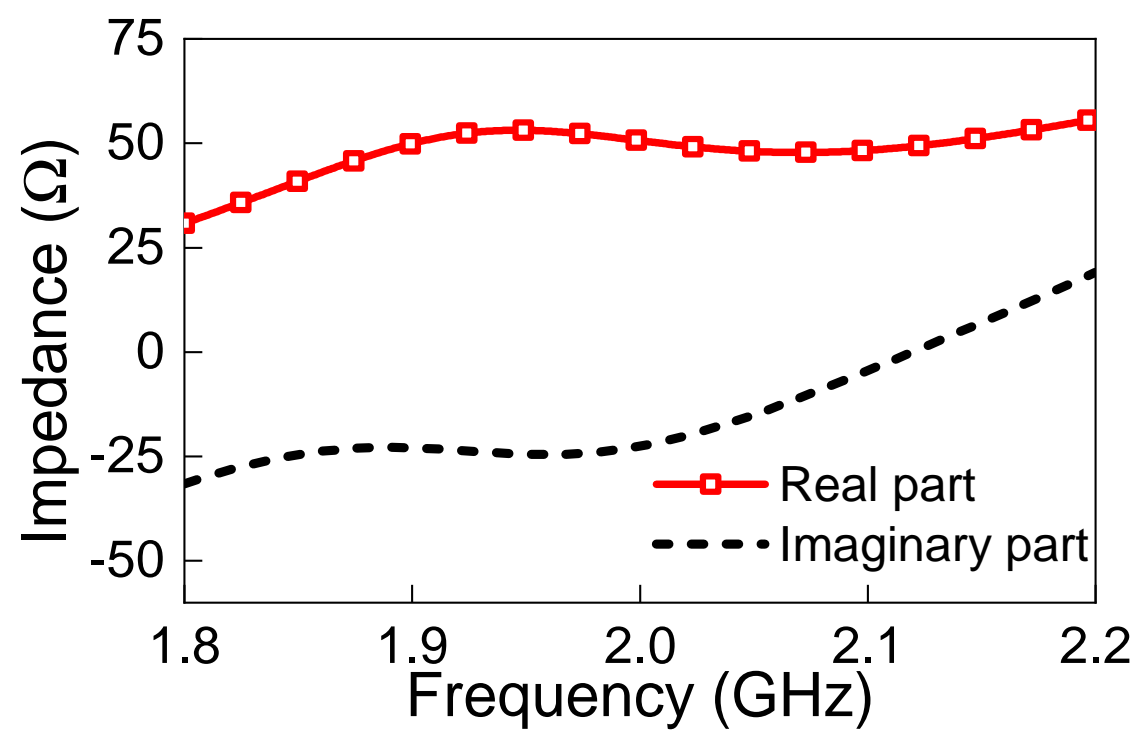

Figure 4.8: Simulated impedance of TPA.

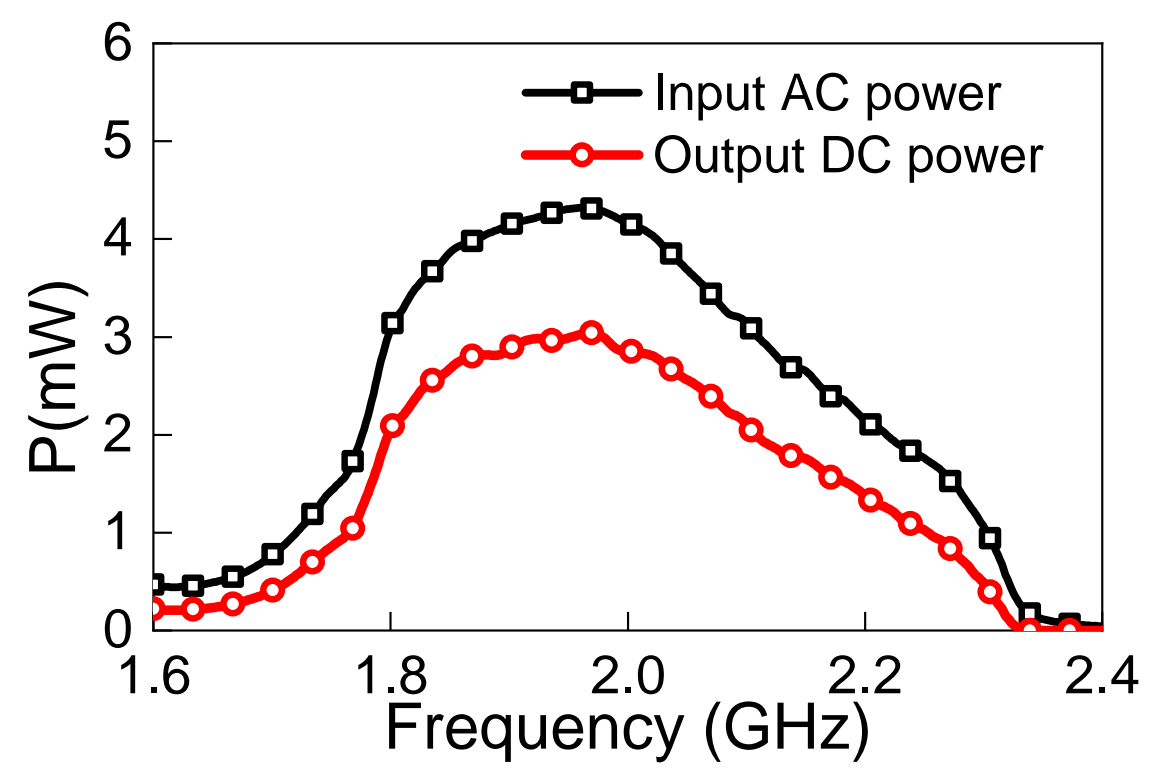

Figure 4.9: Simulated power in $\mathrm{mW}$. 


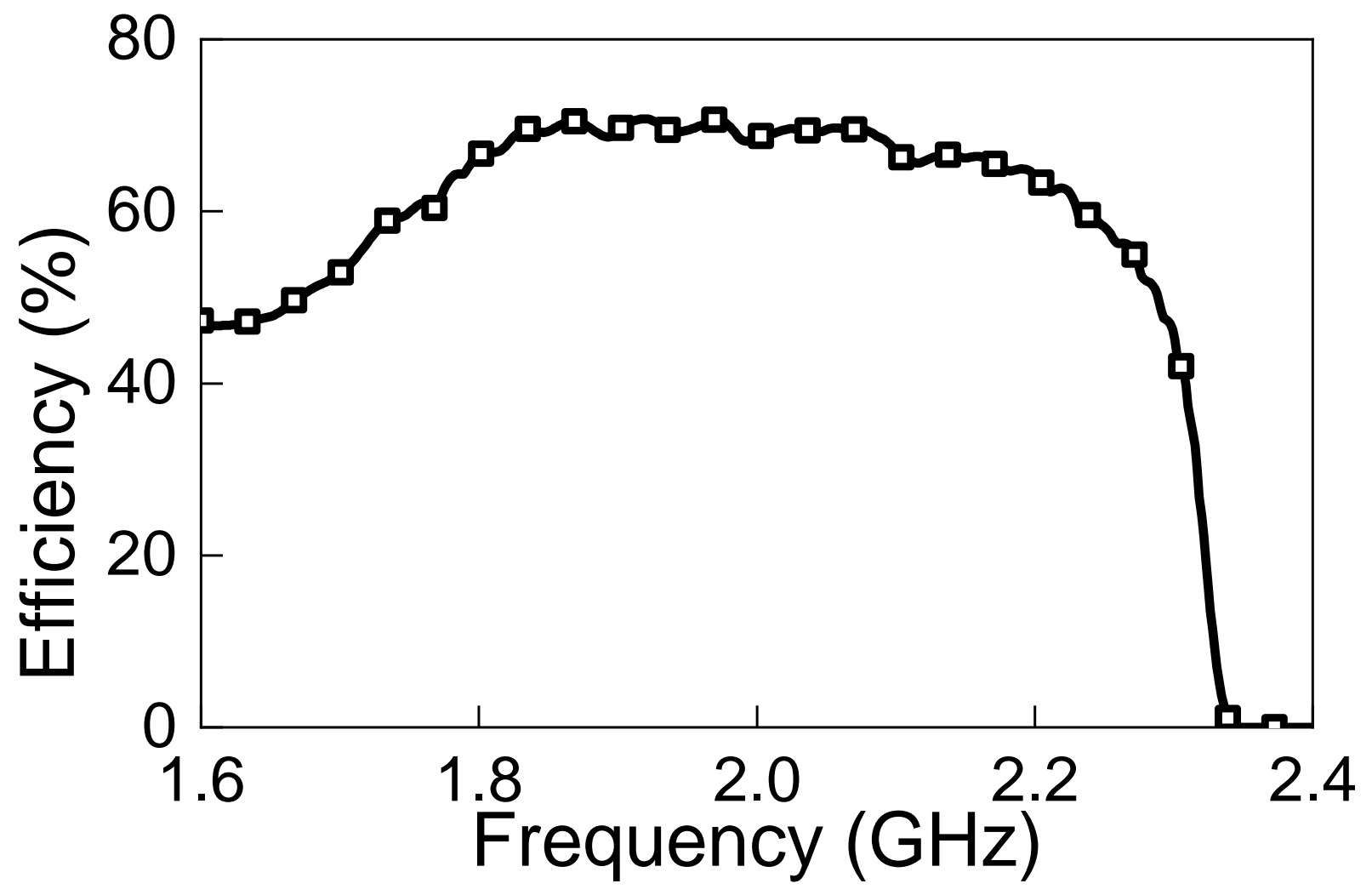

Figure 4.10: Power efficiency $\left(\eta_{a c-d c}\right)$ of the rectifying circuit. 


\subsection{Optimization of Rectenna}

In the literature, the Thevenin equivalent is a well-known technique for replacing the antenna; however, earlier research only looked at the Thevenin equivalent at a single frequency, restricting its application to a fairly narrow frequency range [3, 73, 115]. The capacity to deliver the essential data over the entire working spectrum is the key strength of the method proposed in this work. This section demonstrates how the proposed method can be utilized to optimize the design over any sub-band or the entire operating band.

To enhance the overall efficiency of the rectenna system in particular energy harvesting applications, I may need to improve the design performance within a certain frequency band where the energy concentration is relatively high. Having the entire data about the antenna, I was able to optimize the design illustrated in Fig.4.6 over the required sub-band. Particularly, I divided the entire operating band $\left(R_{T}\right)$ into four equally subbands, namely $R_{1}(1.8-1.9 \mathrm{GHz}), R_{2}(1.9-2 \mathrm{GHz}), R_{3}(2-2.1 \mathrm{GHz})$ and $R_{4}(2.1-2.2 \mathrm{GHz})$. To optimize the design at each sub-band, I adjusted the operating frequency in the harmonic balance simulation controller according to the needed sub-band and ran the optimization tool in ADS. The optimum parameters obtained for each sub-band and the whole band are depicted in Table 4.2 .

Table 4.2:

Parameters of the rectification circuits optimized at various bands $(\mathrm{R})$.

\begin{tabular}{|c|c|c|c|c|c|}
\hline Parameter & $R_{T}$ & $R_{1}$ & $R_{2}$ & $R_{3}$ & $R_{4}$ \\
\hline \hline TL1 $(\mathrm{mm})$ & 14 & 12.8 & 12 & 16.7 & 19.2 \\
\hline TL2 $(\mathrm{mm})$ & 5 & 5.8 & 8.5 & 7 & 3.1 \\
\hline TL3 $(\mathrm{mm})$ & 6.6 & 8.1 & 6.6 & 5.9 & 5.6 \\
\hline TL4 $(\mathrm{mm})$ & 6.2 & 5.2 & 6.4 & 5.8 & 5.8 \\
\hline TL5 $(\mathrm{mm})$ & 5 & 2.9 & 3 & 3.2 & 2.7 \\
\hline TL6 $(\mathrm{mm})$ & 9 & 11 & 9.8 & 8.7 & 8.6 \\
\hline TL7 $(\mathrm{mm})$ & 2 & 2 & 2 & 2 & 2 \\
\hline TL8 $(\mathrm{mm})$ & 2 & 2 & 2 & 2 & 2 \\
\hline TL9 $(\mathrm{mm})$ & 2.2 & 3 & 3.9 & 3.4 & 2 \\
\hline TL10 $(\mathrm{mm})$ & 2.2 & 2 & 2 & 2 & 2 \\
\hline TL11 $(\mathrm{mm})$ & 10 & 12.4 & 10.5 & 10 & 10.1 \\
\hline$R_{L}(\Omega)$ & 700 & 2555 & 2048 & 2254 & 2498 \\
\hline$C_{L}(\mathrm{pF})$ & 100 & 400 & 338 & 232 & 334 \\
\hline
\end{tabular}


Figure 4.11 shows the output DC power for the designs that are optimized at different sub-bands and for the design that is optimized at the entire range of frequency. As illustrated in the Figure, the proposed method enables us to improve the output DC power at required frequency ranges. On the other hand, the results obtained in Fig. 4.11 prove that the lack of data about the entire range limits our ability to optimize the system over the full range. In fact, without the availability of voltage values at all frequencies, optimizing the design across the full band is impossible.

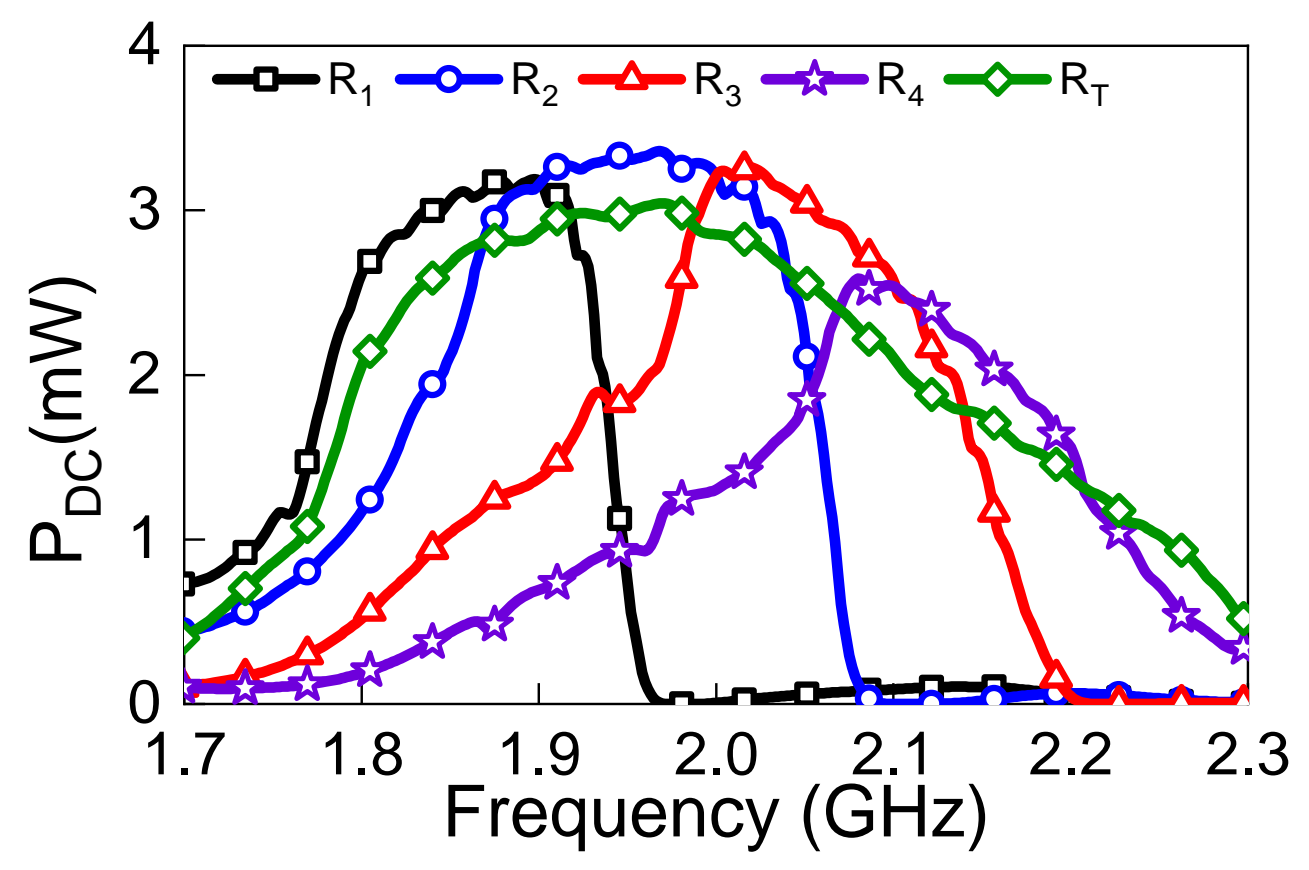

Figure 4.11: Simulated output power in mW optimized at various sub-bands.

\subsection{Validation}

The primary objective of the method presented in this work is to enable full simulation of the rectenna such that the designer can predict accurate performance of the rectenna over the specified frequency range and for any angle of incidence and polarization. Additionally, the method allows for evaluating the performance of any rectenna system designed by a third party, without resorting the measurements. To demonstrate the accuracy of our method, I present a comparison between the simulation results obtained by our method and measurements. To this end, a complete rectenna was designed, fabricated, and the 
DC output voltage was measured. The schematic for the experiment's setup is shown in Fig.4.12. Figure 4.13 shows the laboratory setup. The radiation source comprised a signal generator, a power amplifier and a horn antenna. The receiving side includes the rectenna terminated by a capacitor in parallel with a load resistor. To mimic the simulation setup, I adjusted the output of the signal generator such that the power density at the rectenna was $1 \mathrm{~W} / \mathrm{m}^{2}$. The power density $\mathrm{S}$ at the rectenna can be calculated by [84]:

$$
S=\left(\frac{G_{A} G_{T}(f) P_{T}}{4 \pi R^{2}}\right)
$$

where, $G_{A}$ is the amplifier gain, $G_{T}(f)$ is the gain of the horn antenna at different frequencies obtained from the data sheet, and $P_{T}$ is the output of the signal generator. The distance between the transmitter and receiver, $\mathrm{R}$, is $1 \mathrm{~m}$.

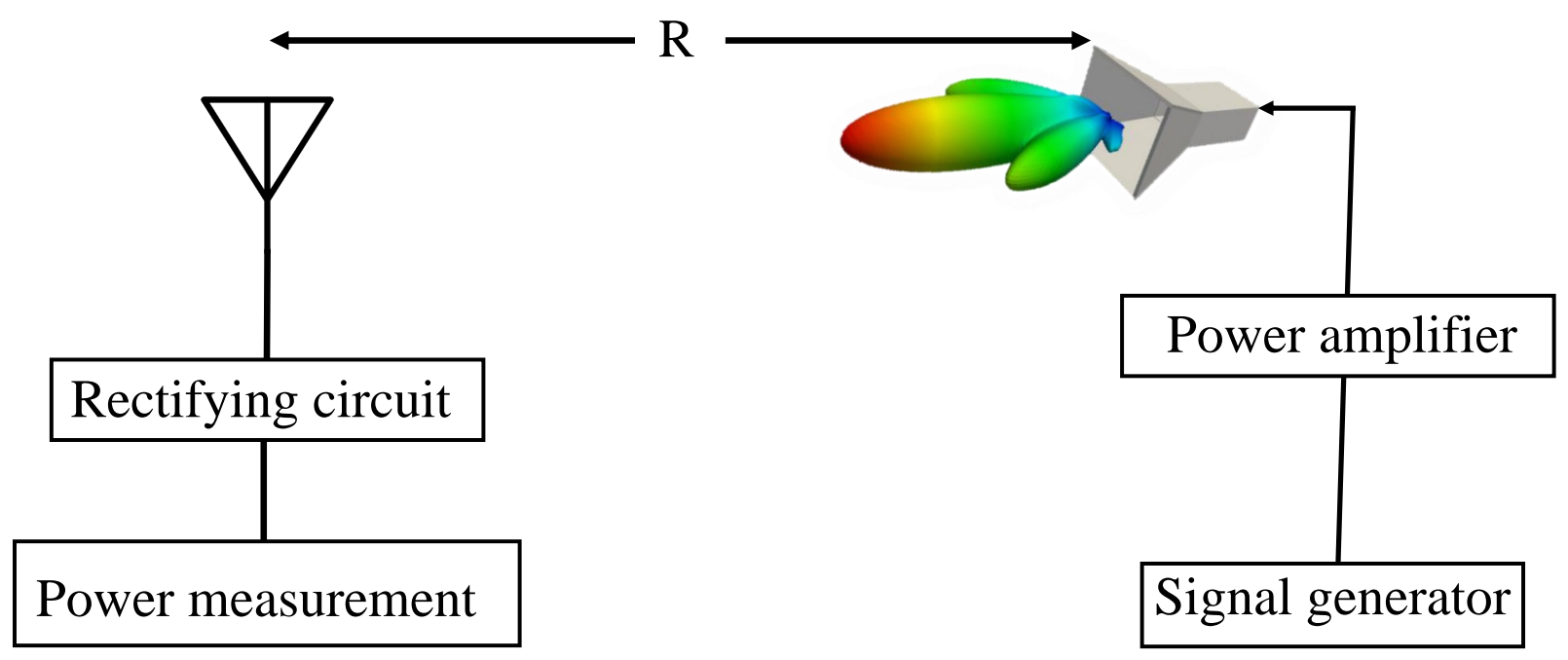

Figure 4.12: Measurements setup; the transmitting side is a horn antenna connected to power amplifier and signal generator and the receiving side is a TPA connected to the rectifying circuit.

In order to measure the output DC power of the system, I terminated the circuit by the same load arrived at earlier using gradient optimization in ADS (a capacitor of $100 \mathrm{pF}$ in parallel with a 700 Ohm resistor). Then the frequency was swept from 1.6 to $2.4 \mathrm{GHz}$ while measuring the DC voltage across the load using a voltmeter. The DC output power was calculated as $\mathrm{P}_{d c}=\mathrm{V}_{L}^{2} / \mathrm{R}_{L}$, where $\mathrm{V}_{L}$ is voltage across the load $\mathrm{R}_{L}$. The results obtained from the measurements and simulations are shown in Fig.4.14. I observe good agreement 


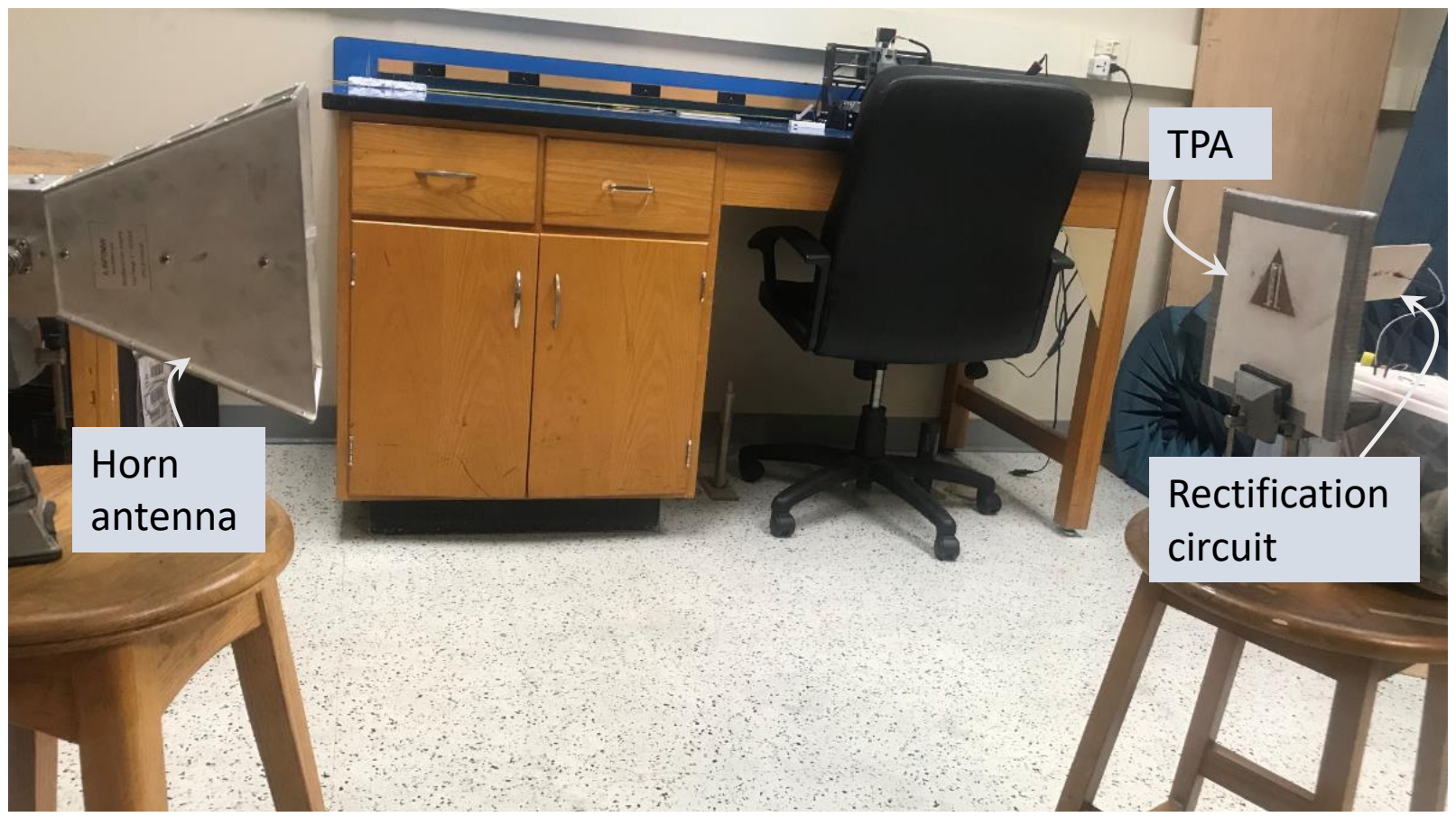

Figure 4.13: Laboratory measurement's setup.

between the measurements and the full-rectenna simulation. I note that the discrepancy between the simulation and measurement is most likely due to fabrication tolerances and especially the laboratory measurements' setup which did not take place inside an anechoic chamber.

In addition to demonstrating the accuracy of our method, I provide a full-rectenna performance comparison between the method presented in this work and the conventional method, which is expected to provide accurate prediction of the DC output power over only a narrow range of frequency and input power (to the rectification circuit) specified during the rectenna design stage. In the conventional method, instead of using a database to change the input voltage with frequency, the maximum input voltage was selected and fixed to provide the circuit with maximum power level at all frequencies as it was done in previous works $[17,80,16]$. (The maximum power level was obtained at $1.95 \mathrm{GHz}$ while the antenna was impedance-matched). From Fig.4.14, I observe, as expected, that the simulation results obtained from the conventional method provide good agreement with measurements only over a very narrow frequency range around the $1.95 \mathrm{GHz}$; however, appreciable deviation from measurements is observed at other frequencies, especially over 
the 1.6 to $1.9 \mathrm{GHz}$ and 2.1 to $2.3 \mathrm{GHz}$ frequency bands.

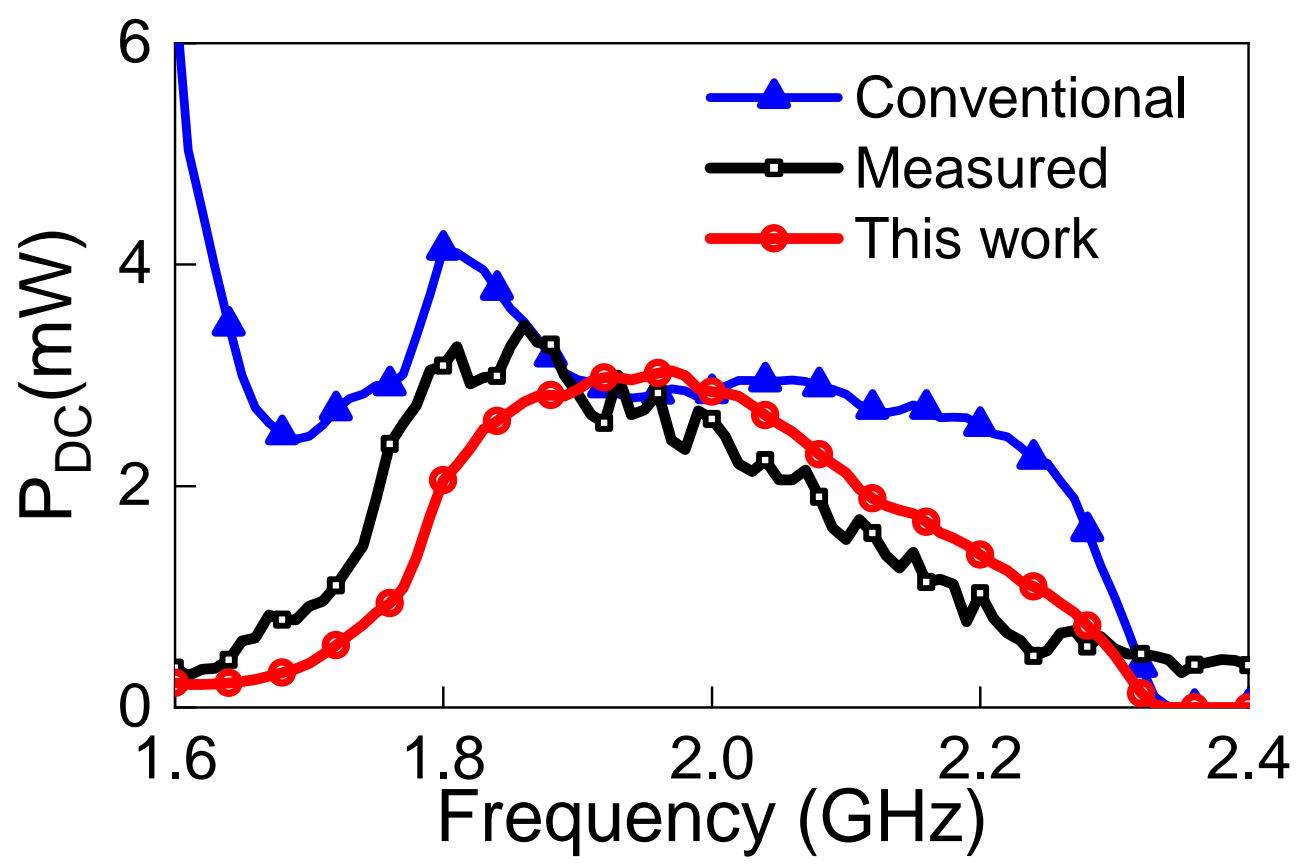

Figure 4.14: Simulated and measured output DC power. The incident field polarization was fixed (y-direction), the incident angle, $\theta$, was fixed at zero degrees (see Fig.4.4), and the power density of the incident field was fixed at $1 \mathrm{~W} / \mathrm{m}^{2}$.

The available power density is an important factor that have an impact on the operation of the overall system. The power density may change due to many reasons such as, but not limited to, the existence of obstacles that block the coming wave, reflections from surrounding environment, and weather conditions. To study the effects of changing the power density on rectenna system, the rectenna was illuminated by a plane wave with different values of power densities. The polarization of the linear plane wave was in the y-direction as shown in Figure 4.4, and the angle of incidence was set to zero (normal incidence from the positive z-direction). Figure 4.15 shows the variation of $V_{o c}$ for different values of power densities (four power density values of $0.25 \mathrm{~W} / \mathrm{m}^{2}, 0.5 \mathrm{~W} / \mathrm{m}^{2}, 1 \mathrm{~W} / \mathrm{m}^{2}$ and $2 \mathrm{~W} / \mathrm{m}^{2}$ were considered). Figure 4.16 shows the effect of changing the power density of the incident field on the output DC power. The non-linear behavior of the rectification circuit is highly visible in the results shown in Fig. 4.16. Utilizing the proposed method, I was able to produce a 3D Figure that displays the fluctuation of the output DC power with frequency and the available power density, as illustrated in Fig.4.17. 


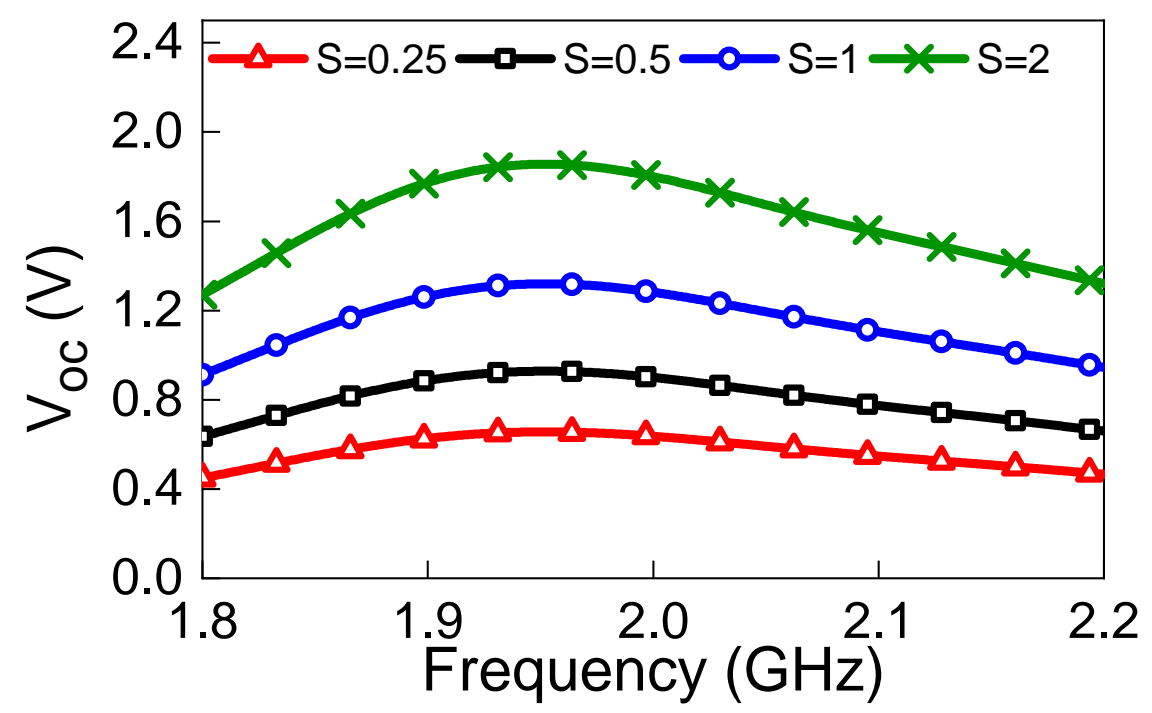

Figure 4.15: Simulated $V_{o c}$ for different power densities of the incident field. The incident field polarization was fixed (y-direction) and incident angle, $\theta$, was fixed at zero degrees (see Fig.4.4).

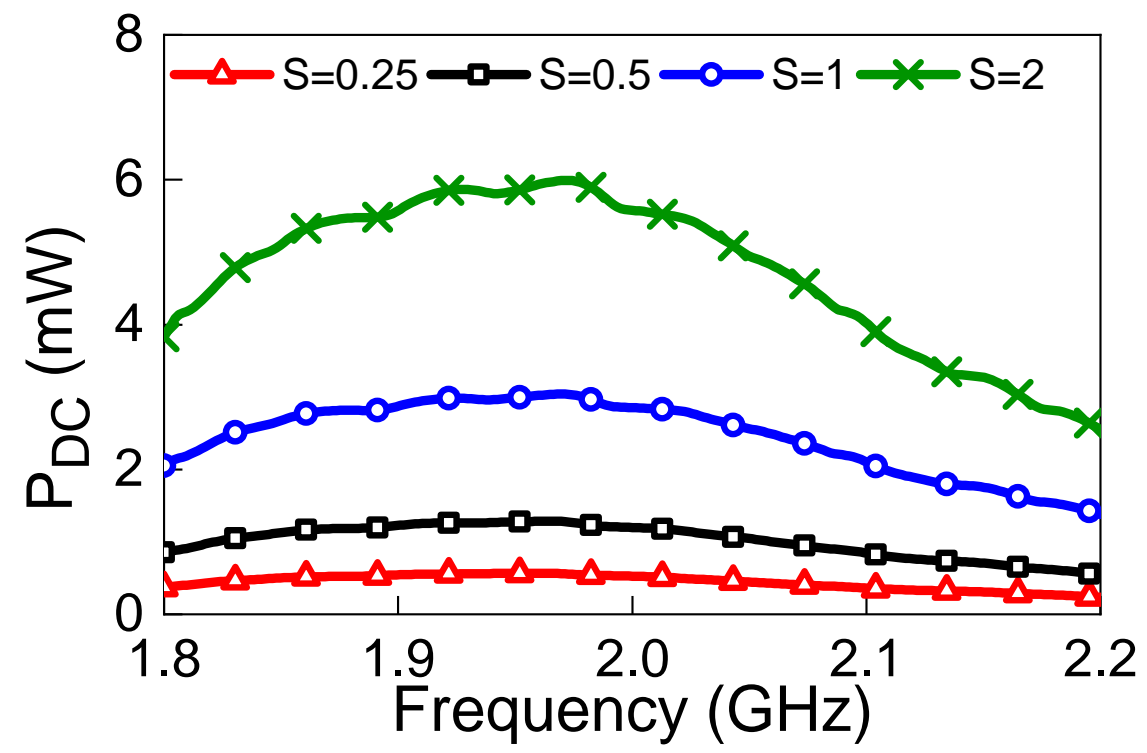

Figure 4.16: Simulated $P_{d c}$ for different power densities of the incident field. The incident field polarization was fixed (y-direction) and incident angle, $\theta$, was fixed at zero degrees (see Fig.4.4). 


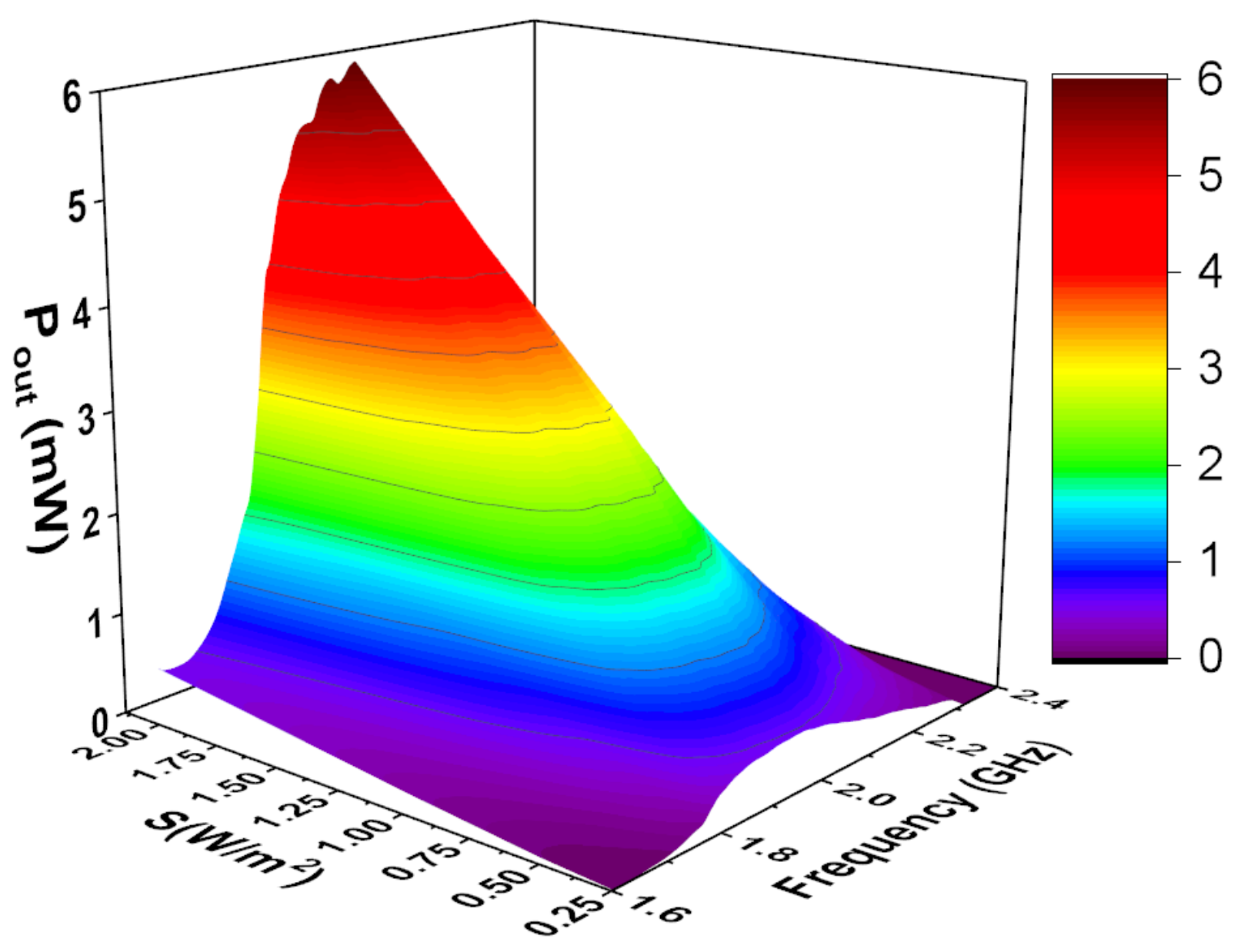

Figure 4.17: Simulated $P_{d c}$ for different power densities of the incident field and different frequencies. The incident field polarization was fixed (y-direction) and incident angle, $\theta$, was fixed at zero degrees (see Fig.4.4). 
To demonstrate the effects of varying the incident angle on the output DC power, I illuminated the rectenna at different incident angles while keeping the power density fixed at $1 \mathrm{~W} / \mathrm{m}^{2}$. The variation in the incident angle does not affect the values of Thevenin impedance, however, it affects $V_{o c}$ for the same power density. To extract the $V_{o c}$ at different angles of incidence, the antenna was illuminated by a linearly polarized (in the y-direction) plane wave. The power density of the incident field was fixed at $1 \mathrm{~W} / \mathrm{m}^{2}$, and the angle of incidence $\theta$ was set to $30^{\circ}, 45^{\circ}$ and $60^{\circ}$. The simulated $V_{o c}$ and output DC power at each angle of incidence are shown in Figs. 4.18 and Fig.4.19. I observe a dramatic enhancement of the output DC power for an incident angle of $60^{\circ}$ over a narrow frequency range of 1.82 to $1.9 \mathrm{GHz}$. Similar behavior is observed for $45^{\circ}$ incidence, but over the narrow frequency range of 1.8 to $1.9 \mathrm{GHz}$. I emphasize that this enhancement is strictly dependent on the topology of the antenna used (TPA in this case). Other types of antennas may not give similar DC power profile. These results, nevertheless, show the importance of providing full predictability of the rectenna system for all types of field excitation (polarization, power density, and angle of incidence). In fact, the simulation method presented in this work enables the production of a comprehensive graphical chart that shows the performance of the rectenna system under any excitation condition. Furthermore, I was able to create a 3D Figure using the provided method that shows the variation of the output DC power with frequency and angle of incident, as shown in 4.20.

The extracted values of $V_{o c}$ for each angle of incidence are loaded to the source of the circuit shown in Fig.4.6. As depicted in the Figure, the variation of incident angle has a direct impact on the output DC power. The variation in the output DC power due to change in incident angle are identical to that in $V_{o c}$. 


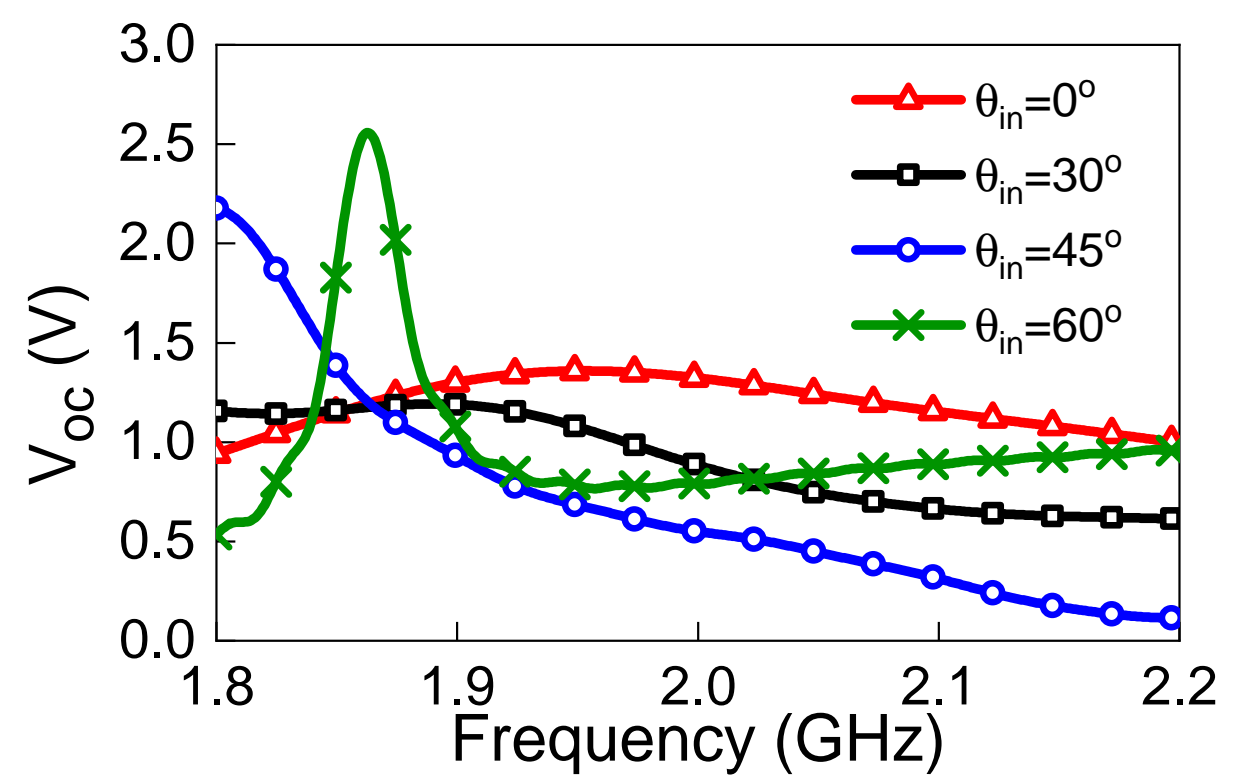

Figure 4.18: Simulated $V_{o c}$ values for different angles of incidence. The power density of the incident field was kept constant at $1 \mathrm{~W} / \mathrm{m}^{2}$.

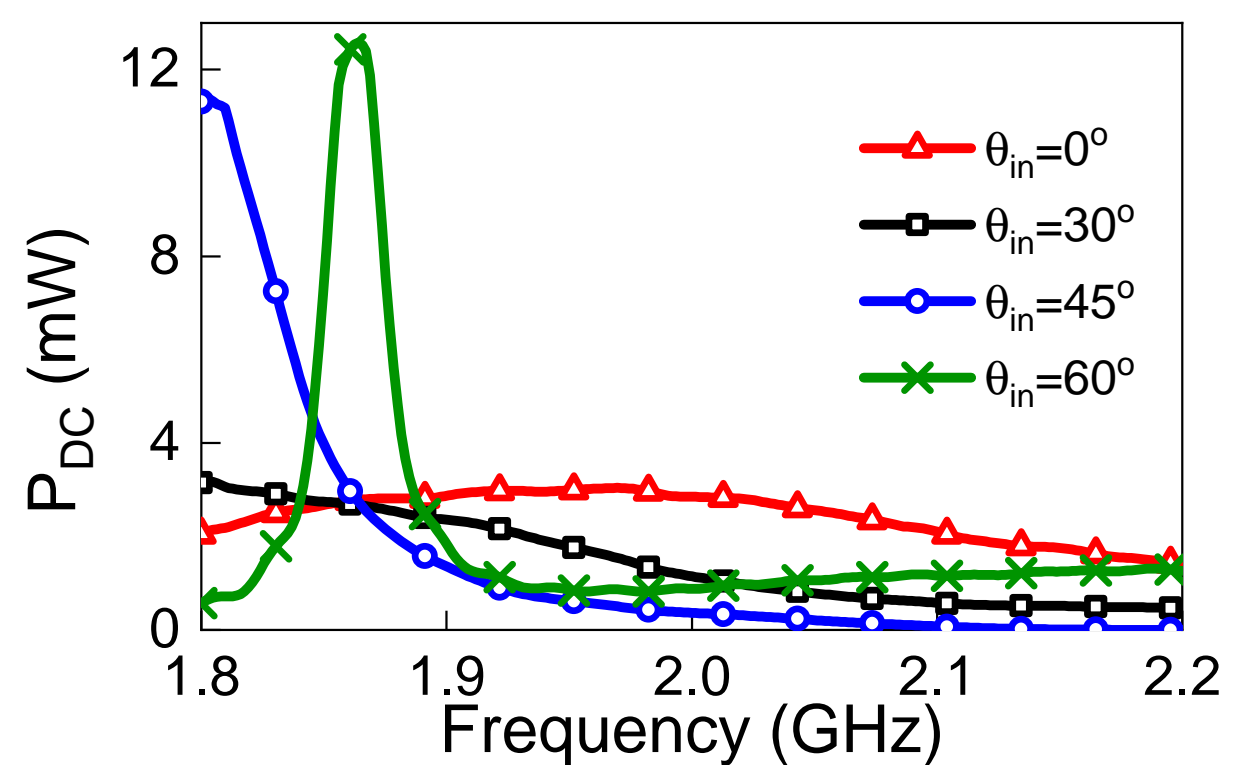

Figure 4.19: Simulated $P_{d c}$ for different angles of incidence. The power density of the incident field was kept constant at $1 \mathrm{~W} / \mathrm{m}^{2}$. 


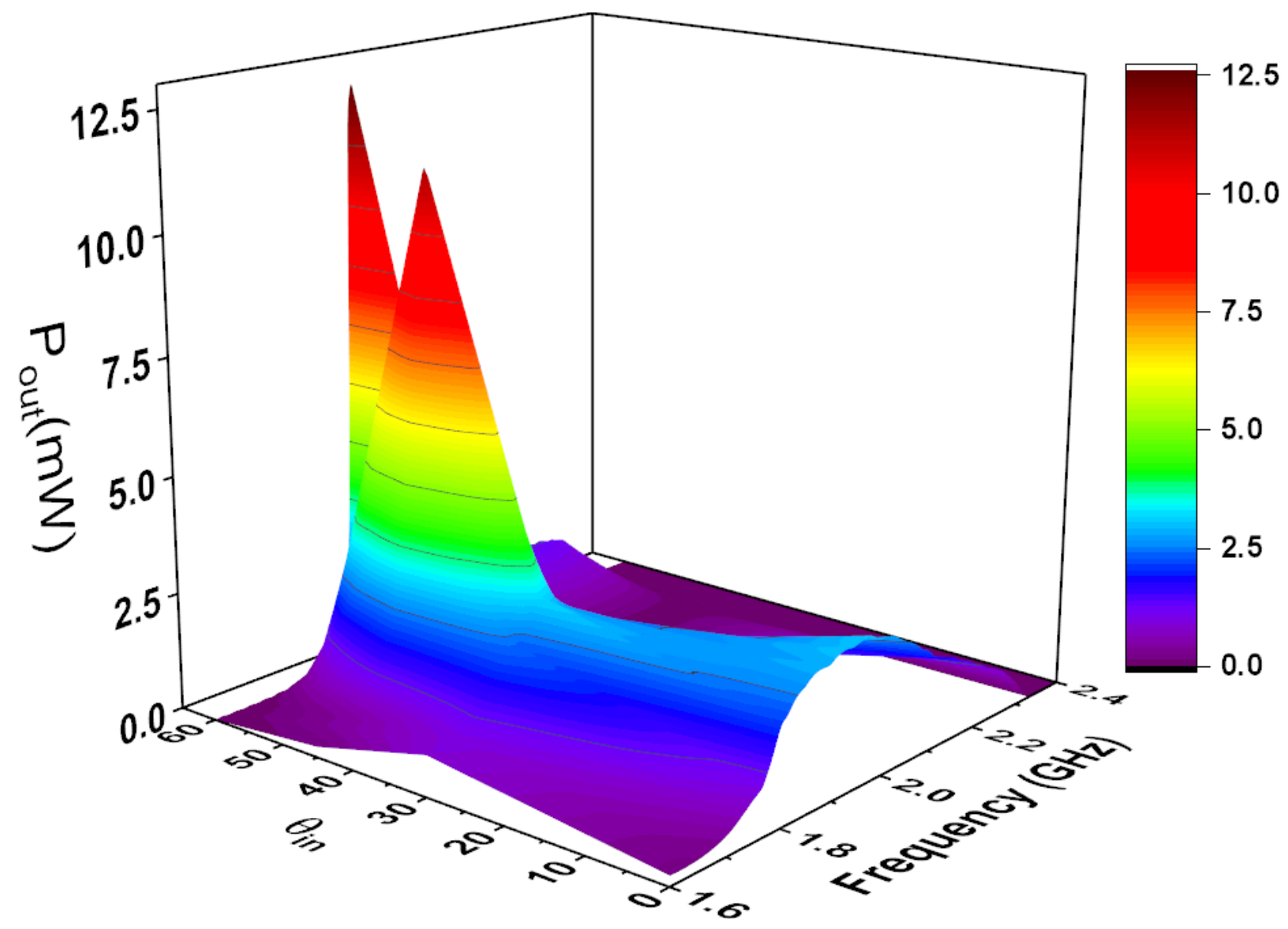

Figure 4.20: Simulated $P_{d c}$ for different angles of incidence and different frequencies. The power density of the incident field was kept constant at $1 \mathrm{~W} / \mathrm{m}^{2}$. 


\subsection{Conclusion}

In this chapter, I proposed a method to enable full simulation or characterization of a complete rectenna. This is accomplished by replacing the receiving antenna by its Thevenin equivalent circuit, and then using a circuit simulator after incorporating the equivalent circuit with the rectification circuit and load.

The primary objective of our method is to enable full simulation of the rectenna such that the designer can predict accurate performance of the rectenna over the specified frequency range and for any angle of incidence and polarization. This all can be accomplished without fabrication and measurements. In fact, using our simulation method, one can produce a comprehensive graphical chart that shows the performance of a specific rectenna under any excitation condition (polarization, incidence angle, and power density). This feature enables the evaluation of the performance of any rectenna designed by a third party without resorting to measurements.

Another advantage of our method is its ability to optimize the rectification circuit for a specific receiving antenna to deliver maximum DC power. This advantage is critical since an optimal rectification circuit is strongly dependent on the particular antenna employed. Furthermore, utilizing this method, I was able to optimize the rectenna at various subbands of the operating spectrum. 


\section{Chapter 5}

\section{Metasurface Antenna (MSA)}

\subsection{Introduction}

Metamaterials have been viewed as an artificial materials with electromagnetic properties that cannot be obtained naturally [116, 81]. Fundamentally, the metamaterials are identified as a structure composed of electrical small resonators that create an electromagnetic media with preferable characteristics. The resonators can have many shapes such as spheres, wires, split ring resonators (SRRs) and C-shape. Metamaterials are hypothesized and experimentally realized as a material with negative permittivity and negative permeability simultaneously [117]. To construct a metamaterial, periodic structures of resonators that operate collectively as an effective medium are utilized [82]. These resonators can be tailored to provide the required properties. Metamaterials have been utilized in wide range of applications including, but not limited to, energy harvesting [118], advance lenses [119], cloaking [120], metamaterial-based antenna [82].

Metamaterials can be realized in many shapes and dimensions. Different types of metamaterial are available such as, electromagnetic bandgap (EBG) [121], High impedance surfaces (HIS) [122], and metasurface (MS) [82]. Characteristics like low cost, small size, high efficiency, wide bandwidth are desired in wide range of applications. These characteristics can be provided by metasurface-based antenna. Metasurface is a two dimensional type of metamaterial in which the structure occupying very thin surface (metafilm) [91].

In this chapter, I present a design of a Metasurface Antenna (MSA) utilizing electrical small elements to resonate strongly to resemble the resonance of classical circuits. Facilitated by matching due to coupling, the small radiators collectively provide a good radiation 
characteristics. The performance of the proposed MSA is compared with an MPA array design. Additionally, a rectenna system that utilize the MSA as receiving part is devel-

oped and investigated. Finally, the capability of the MSA to operate in the $5 \mathrm{G}$ band is investigated.

\subsection{MSA}

\subsubsection{MSA Design}

The electrical ring resonator (ERR) used in this work have already been reported in [82] were employed as electrically-small resonators to demonstrate the concept of MSA. I built a periodic structure of the ERRs to operate as a sub-wavelength particles that resonate strongly to electric field and show negligible response to the magnetic field. To guarantee optimal antenna gain and bandwidth, I developed a feeding network that connects all radiators to a single feeding point. In this study, all simulation results are carried out by using CST MWS software tool [114].

Fig. 5.1 shows the structure layers of the MSA and the feeding network. Observing Fig. 5.1 , the top layer of the MSA is made of $6 \times 8$ identical cross strip resonators designed and optimized to operate around $5.8 \mathrm{GHz}$. The ground is sandwiched between two identical layers of a semi-flexible Roger material (RT5880) with a thickness of $0.787 \mathrm{~mm}$, a relative permittivity of $\varepsilon_{\mathrm{r}}=2.2$ and a loss tangent of $\tan \delta=0.009$. The radiating elements are printed on the top of the Roger material and connected by vias to channel the current to a feeding network. The diameter of the via is $0.5 \mathrm{~mm}$ due to the fabrication constraint. The via position is optimized to provide reflection coefficient less than $-10 \mathrm{~dB}$ at the operating frequency. Taking into account the effect of spacing between cells, the optimal resistance value of each resonator is $250 \Omega$.

To eliminate the coupling between the adjacent elements, the separation between elements of antenna array usually is half wave length [82, 83, 108]. However, the separation between radiating elements of this design is electrically small to show matching with their feeding port. The small separation distance between radiating elements necessitates the use of feeding network on a separated layer to connect all elements to one feeding port.

When a load's impedance is properly matched to the transmission line's characteristic impedance, all transmitted power is dissipated in the load and there is no reflected wave. Impedance matching can be accomplished in a variety of methods, such as using a quarterwave transformer, single stub tuning, or double stub tuning. 


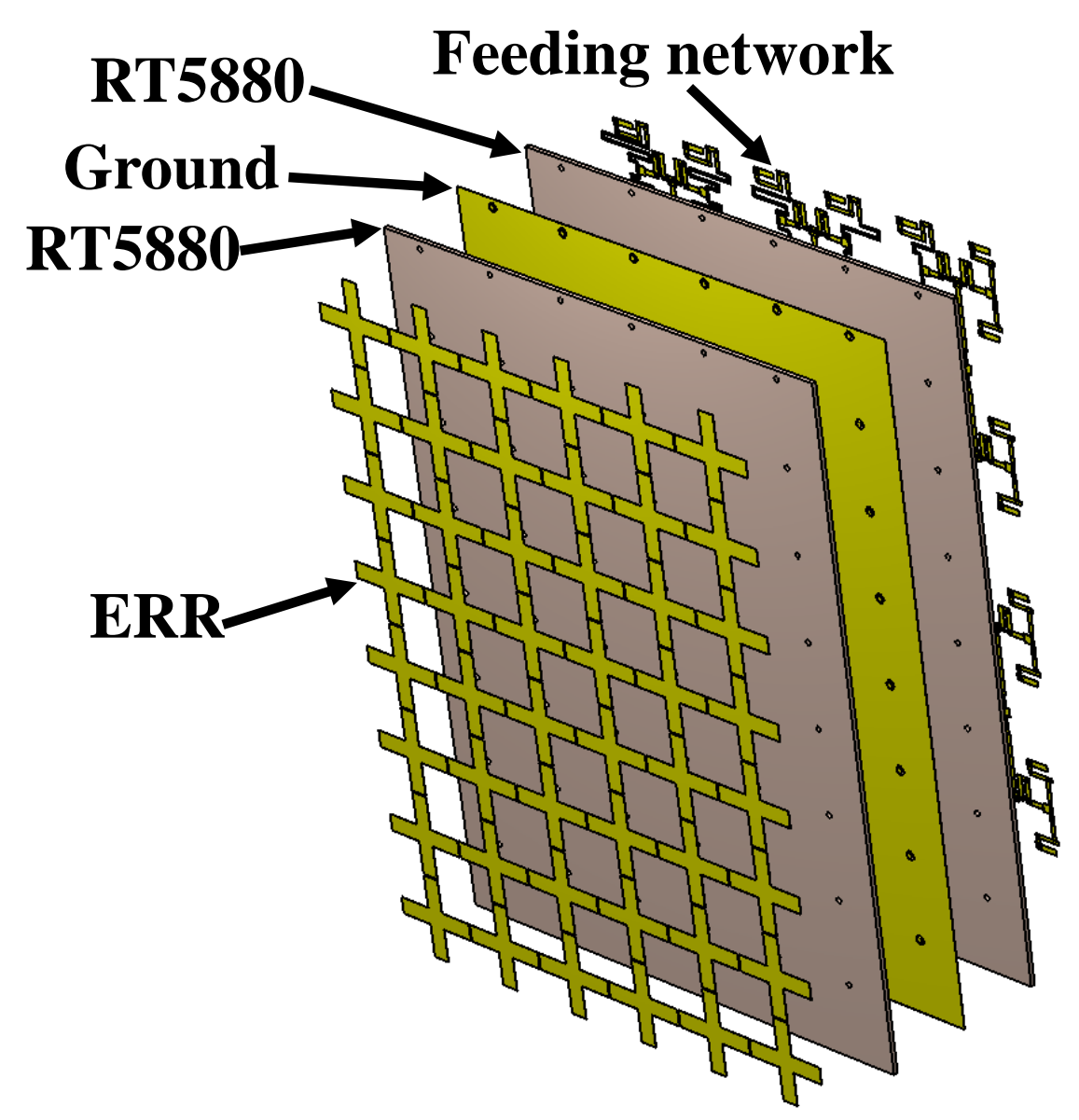

Figure 5.1: Metasurface structure

\subsubsection{Feeding Network Design}

To design the feeding network shown in Fig. 5.2a, I utilized the transmission lines as quarter-wave transformer. A quarter wave transformer alters the load's impedance to a different value, allowing for matching utilizing a transmission line of $\lambda / 4$ length as shown in Fig. 5.2b. To satisfy the matching condition in a quarter wave transformer, I need $Z_{\text {in }}=Z_{o}$. To calculate the characteristic impedance $\left(Z_{T}\right)$ of the $\lambda / 4$ section illustrated in Fig. 5.2b:

$$
Z_{\text {in }}=Z_{T} \frac{Z_{L}+j Z_{T} \tan \frac{\pi}{2}}{Z_{T}+j Z_{L} \tan \frac{\pi}{2}}=\frac{Z_{T}^{2}}{Z_{L}}
$$


since $\tan \beta l=\tan \frac{2 \pi}{\lambda} \frac{\lambda}{4}=\tan \frac{\pi}{2}=\infty$. In order for $Z_{\text {in }}=Z_{0}$, I need that

$$
Z_{T}^{2}=Z_{0} Z_{L} \Rightarrow Z_{T}=\sqrt{Z_{0} Z_{l}}
$$

Utilizing transmission lines as quarter-wave transformers, I connected all 48 cells to one port of $50 \Omega$ as depicted in Fig.5.2a. The feeding network is optimized to collect maximum power from all elements at the operating frequencies.

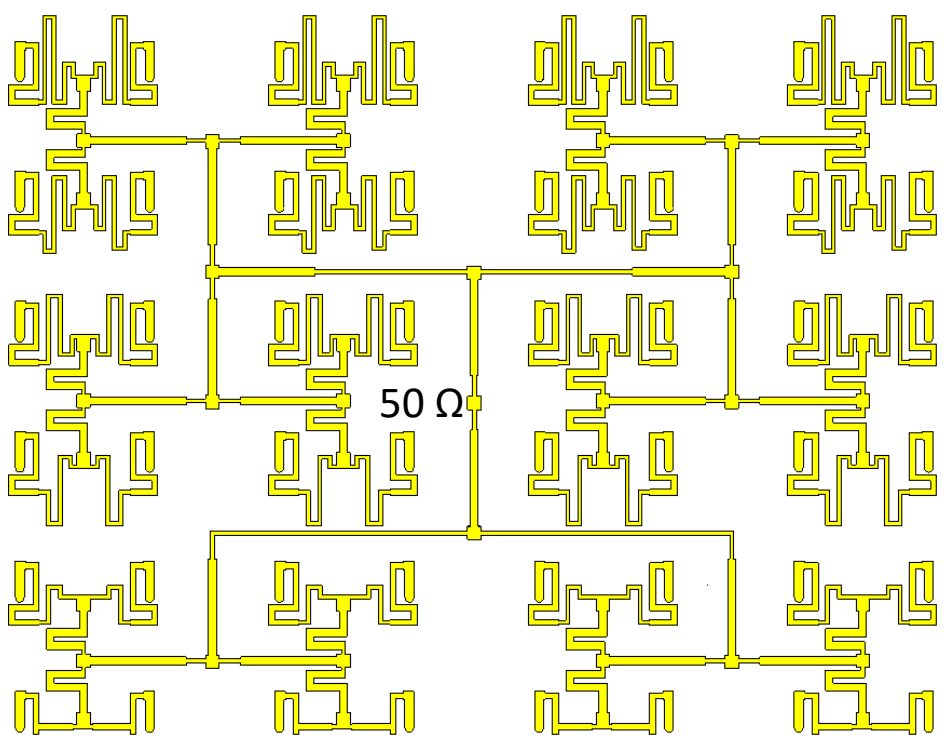

(a)

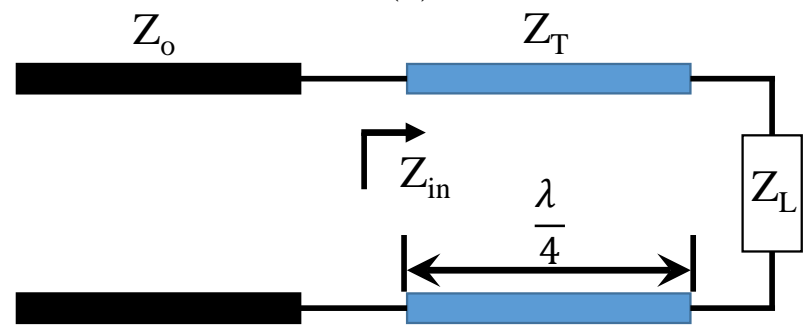

(b)

Figure 5.2: (a) The feeding network of the MSA. (b) Quarter-wave transformer. 


\subsubsection{Results of the Proposed MSA}

The design was fabricated and tested for validation. Figure 5.3a displays the top view of the fabricated design and Figure 5.3b shows the bottom view of the realized design. Fig.5.4a illustrates the measured and the simulated reflection coefficient of the MSA. It can be observed that, the MSA shows reflection coefficient of less than $-10 \mathrm{~dB}$ at around 5.8 $\mathrm{GHz}$, with an impedance bandwidth response from 5.66 to $5.85 \mathrm{GHz}$. The measurement results show wider band than the simulation results. This difference could be due to the port soldering. Furthermore, the gain and the efficiency of the design were studied numerically and measured over the operating frequency. Fig.5.4b depicts the simulation and the measurement results of the gain and the efficiency of the design. The gain is ranging from 9.5 to $13 \mathrm{dBi}$ over the operating spectrum, and the efficiency is ranging from $59 \%$ to $81 \%$ as illustrated in the Figure. In both the gain and the efficiency, there was good agreement between the measurement and simulation results.

Figures 5.5a and 5.5b illustrate the MSA's 2D normalized radiation pattern in the $\mathrm{H}$ and E planes respectively at $5.8 \mathrm{GHz}$. At the given frequency, the simulated and measured results are in good agreement. As illustrated in Fig.5.5a, the major H-plane loop is in the forward direction. In E-plane, the MSA shows an omni-directional pattern as depicted in Fig.5.5b.

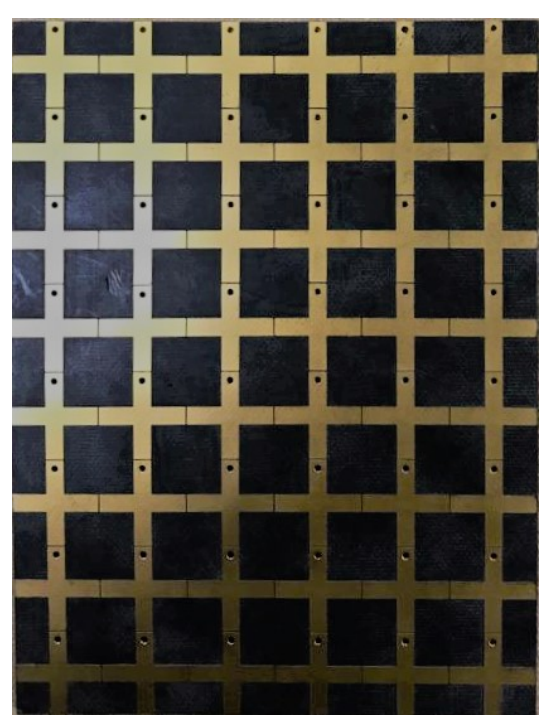

(a)

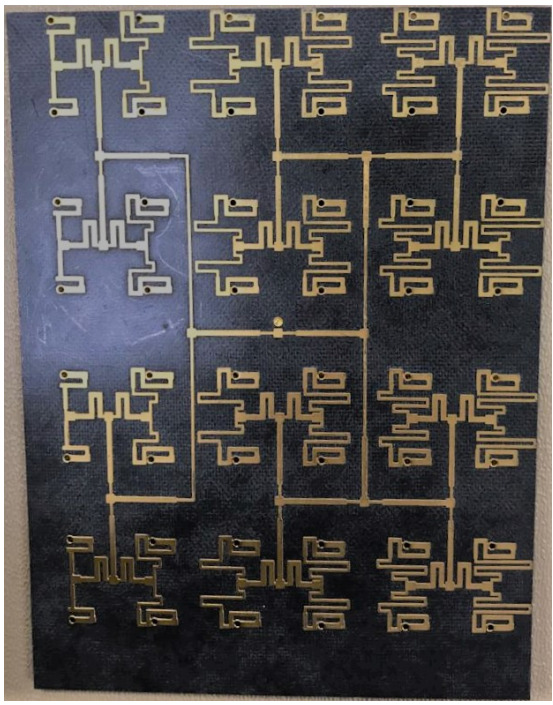

(b)

Figure 5.3: Realization of the MSA: (a) ERR (Top) (b) Feeding network (Bottom). 


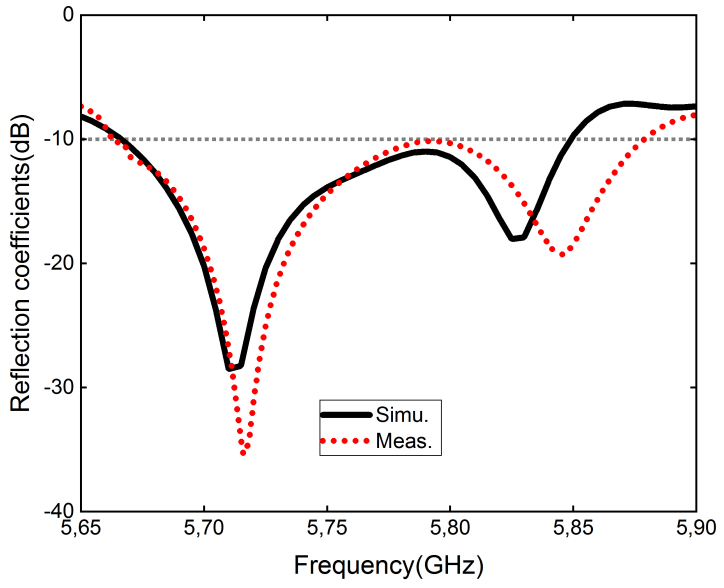

(a)

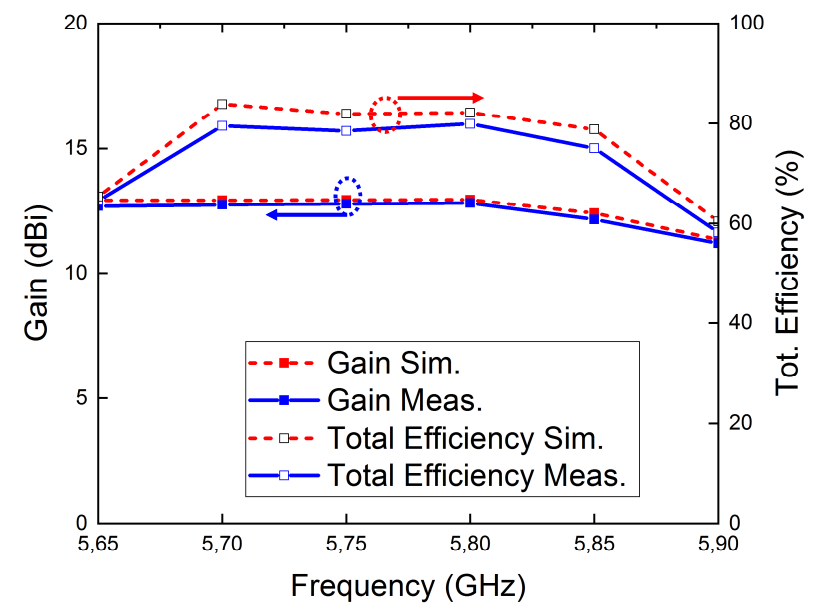

(b)

Figure 5.4: (a) Simulated and measured reflection coefficients of the proposed MSA. (b) Simulated and measured gain and efficiency of the proposed MSA.

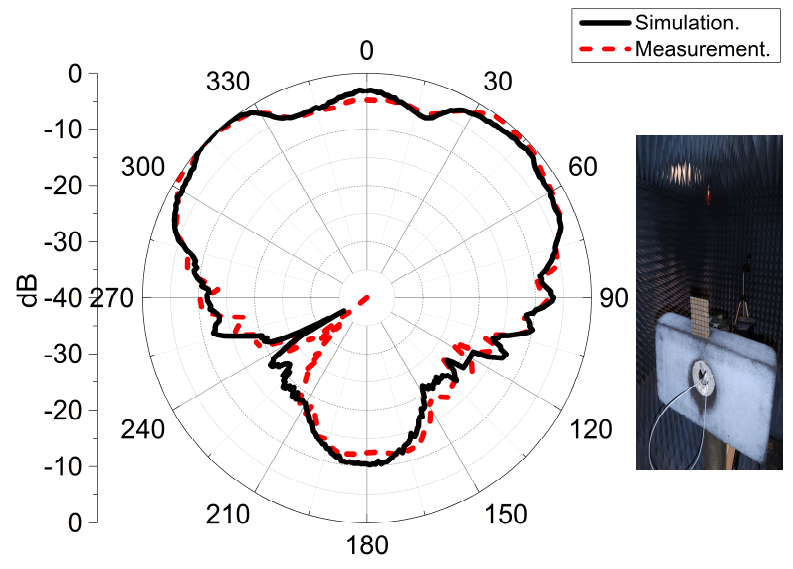

(a)

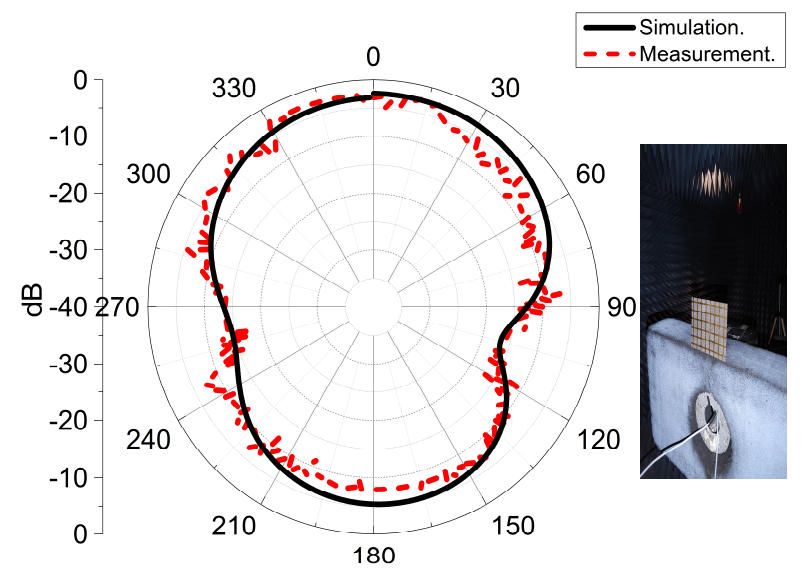

(b)

Figure 5.5: Simulated and measured normalized radiation patterns of proposed MSA at $5.8 \mathrm{GHz}$. (a) H-plane. (b) E-plane. 


\subsection{Effect of Bending on MSA and MPA Array}

\subsubsection{Introduction}

Recently, the growth of wireless technologies and applications requires antennas with multiple capabilities and functions. Characteristics like low cost, small size, high efficiency, and wide bandwidth are desired in many technologies. Furthermore, antennas that can be used both as flat and as curved (conformal antennas) are becoming an increasingly attractive antennas because of their wide range of application [81]. To compare their sensitivity to bending, multiple numerical studies have been performed on MSA and MPA array designs at different curvatures. To make a fair comparison, both designs have the same footprint $\left(120 \times 90 \mathrm{~mm}^{2}\right)$. The dimension of designs are optimized to operate in the Industrial, Scientific, and Medical (ISM) band with a center frequency of $5.8 \mathrm{GHz}$.

\subsubsection{Comparison Results}

In this section, I compare the performance of an MSA and an MPA array when they are flat and curved. For MSA, I utilized the MSA described in Fig.5.1. For MPA array, I designed an array of MPA utilizing same material and same foot print of MSA. Unlike the MSA, each element of MPA array can operate effectively. To increase the gain of a single element MPA, several elements are utilized to form an MPA array [83]. To avoid coupling between the radiating elements in an MPA array, the distance between the elements should be equal to or larger than half wave length $(\lambda / 2)$. In this study, all simulation results are carried out by Computer System Technology (CST) numerical simulator tool [114].

As shown in Fig.5.6, the MPA array is $4 \times 2$ elements of square patch antenna operating around $5.8 \mathrm{GHz}$. Each element has a side length of $16.8 \mathrm{~mm}$, and fed at $0.2 \mathrm{~mm}$ from the center of the radiating element to show good matching to $100 \Omega$ port at $5.8 \mathrm{GHz}$. Utilizing transmission lines as quarter-wave transformer, a feeding network with a single $50 \Omega$ port is incorporated on the same layer of the radiating elements.

As illustrated in Fig. 5.7a, the MSA shows reflection coefficient of less than $-10 \mathrm{~dB}$ at frequencies of 5.66 to $5.85 \mathrm{GHz}$. Compared with MSA, the MPA array shows a matching at narrower range of frequencies ranging from 5.75 to $5.85 \mathrm{GHz}$. Figure 5.7b shows the the gain and the radiation efficiency of the MSA and the MPA array. The realized gain of the MSA is ranging from 9.5 to $13 \mathrm{dBi}$ over the operating range, and the total efficiency is ranging from 48 to $81 \%$. On the other hand, the realized gain of the MPA array is ranging from 11 to $12 \mathrm{dBi}$, and the total efficiency is ranging from 70 to $95 \%$. 


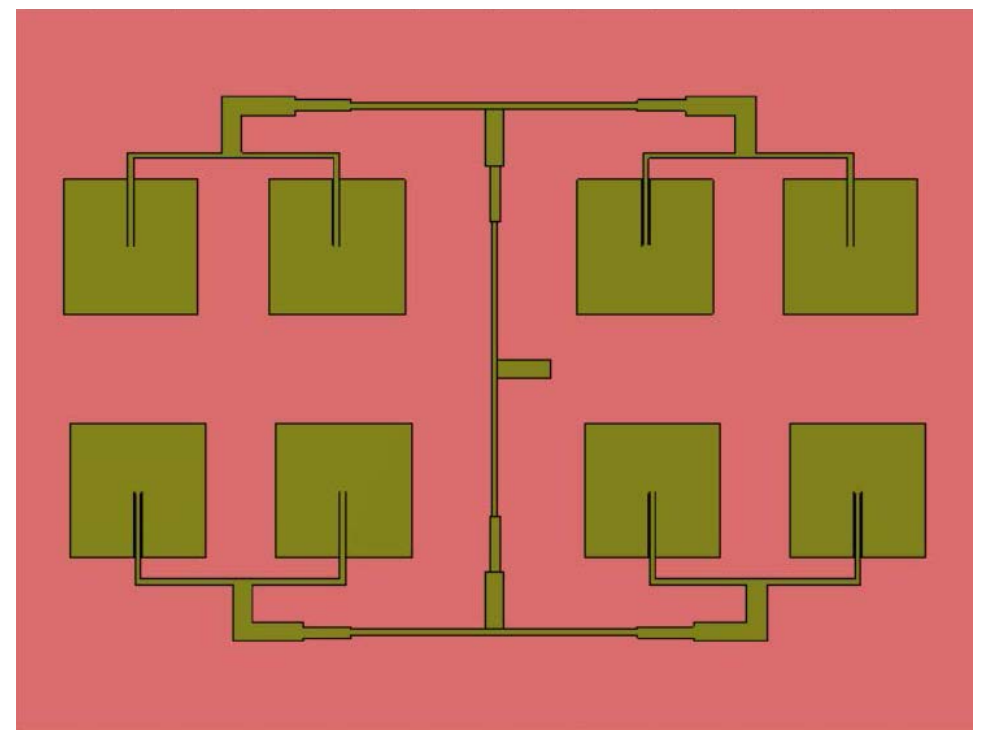

Figure 5.6: Top view of MPA array.

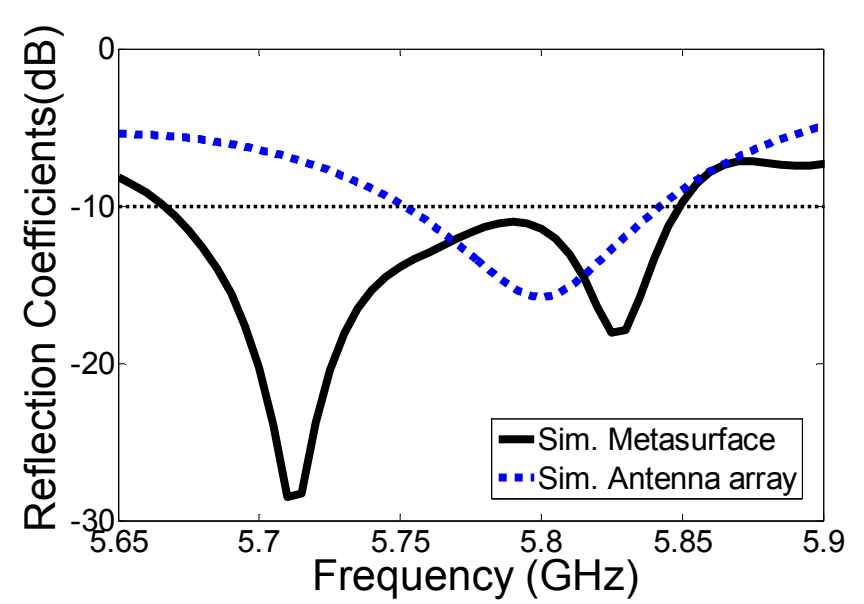

(a)

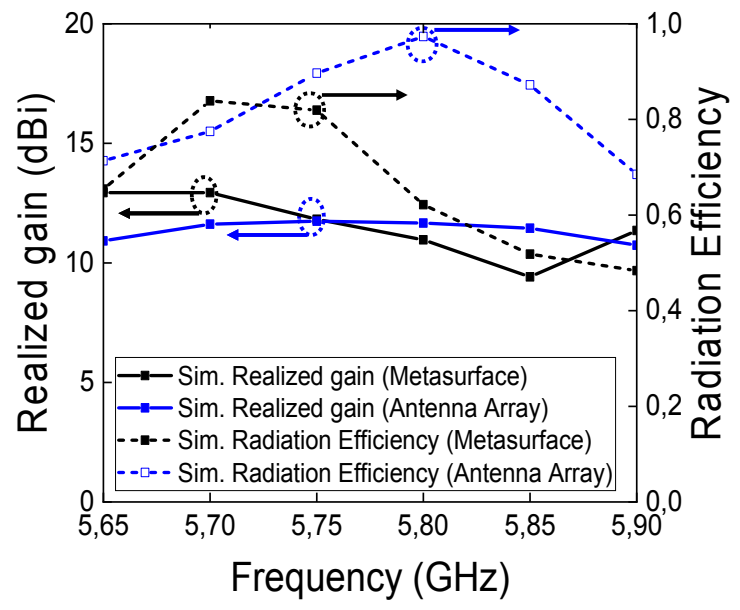

(b)

Figure 5.7: (a) Reflection coefficient of MSA and MPA array. (b) Efficiency and gain of MSA and MPA array. 
To compare the sensitivity of both designs to bending, I evaluated their performance at three different curvatures with radii of 50,75, and $100 \mathrm{~mm}$. Figure 5.8 shows the deformed structure of MSA at 3 different radii of 50,75 , and $100 \mathrm{~mm}$. The deformed structure of MPA array is illustrated in Figure 5.9. The reflection coefficient of the MSA and the MPA array is numerically studied. As illustrated in Fig. 5.10a, the resonant frequency of the MSA is slightly changed due to the bending. At all curvatures, the design remains within its operational spectrum $(5.75-5.85 \mathrm{GHz})$. The resonant frequency of the MPA array is extremely sensitive to the bending. As depicted in Fig.5.10b, bending the MPA array caused the resonance frequency to shift from $5.8 \mathrm{GHz}$ to $5.3 \mathrm{GHz}$.

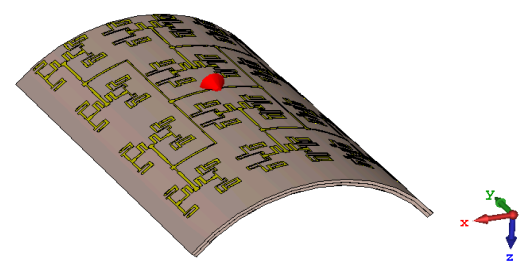

(a)

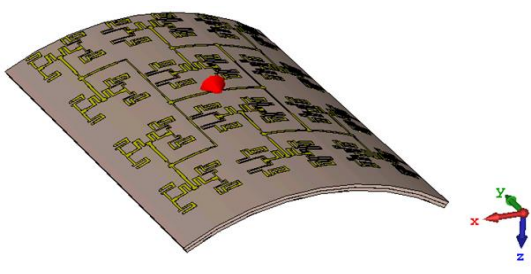

(b)

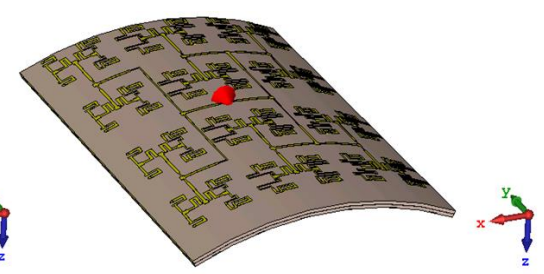

(c)

Figure 5.8: Structurally deformed MSA with different values of radius: (a) $\mathrm{R}=50 \mathrm{~mm}$, (b) $\mathrm{R}=75 \mathrm{~mm}$, (c) $\mathrm{R}=100 \mathrm{~mm}$.

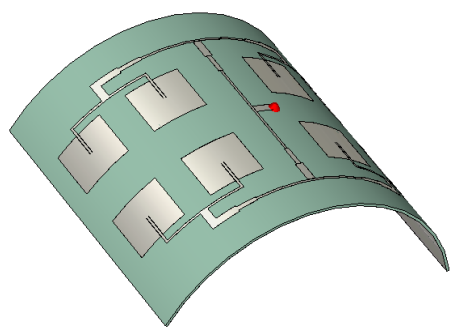

(a)

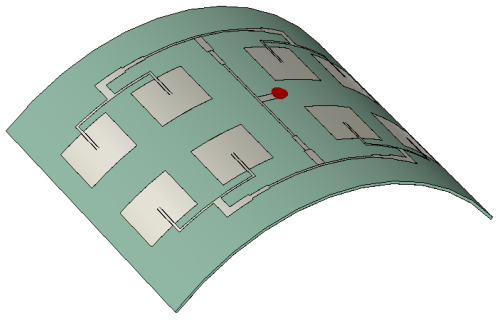

(b)

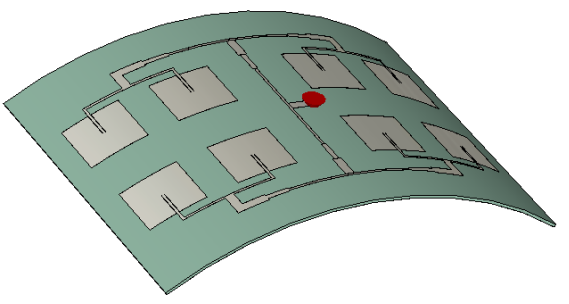

(c)

Figure 5.9: Structurally deformed MPA array with different values of radius: (a) $R=50$ $\mathrm{mm}$, (b) $\mathrm{R}=75 \mathrm{~mm}$, (c) $\mathrm{R}=100 \mathrm{~mm}$.

Figure 5.11a shows the variation of the realized gain of MSA with bending. It can be noted that, bending the design at different curvatures slightly increases the gain in the frequency ranging from 5.75 to $5.85 \mathrm{GHz}$. On the other hand, as I increases the curvature of MPA array the gain is decreasing for the same range of frequency as illustrated in Fig. 5.11b. For the same frequency range, the MSA shows stability in the gain at different curvatures, whereas the MPA array shows a high sensitivity in the gain to curvature change. 


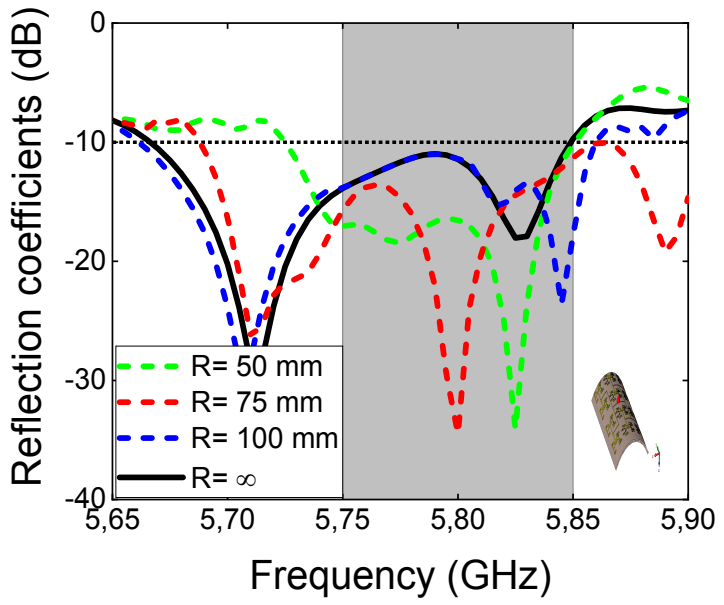

(a)

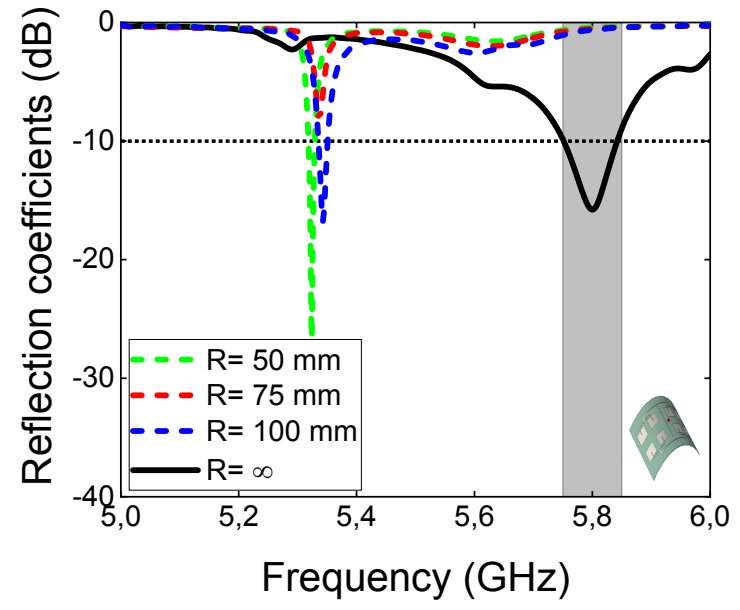

(b)

Figure 5.10: (a) Reflection coefficient of MSA at different curvatures. (b) Reflection coefficient of MPA array at different curvatures.

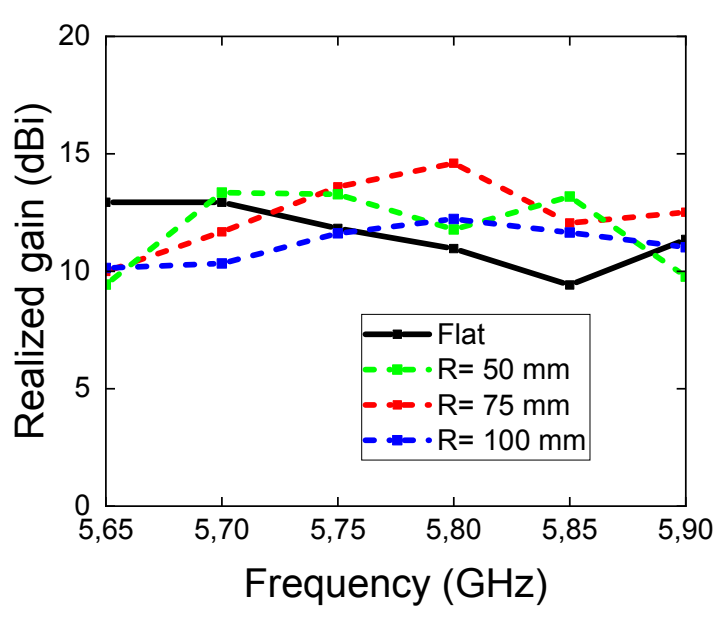

(a)

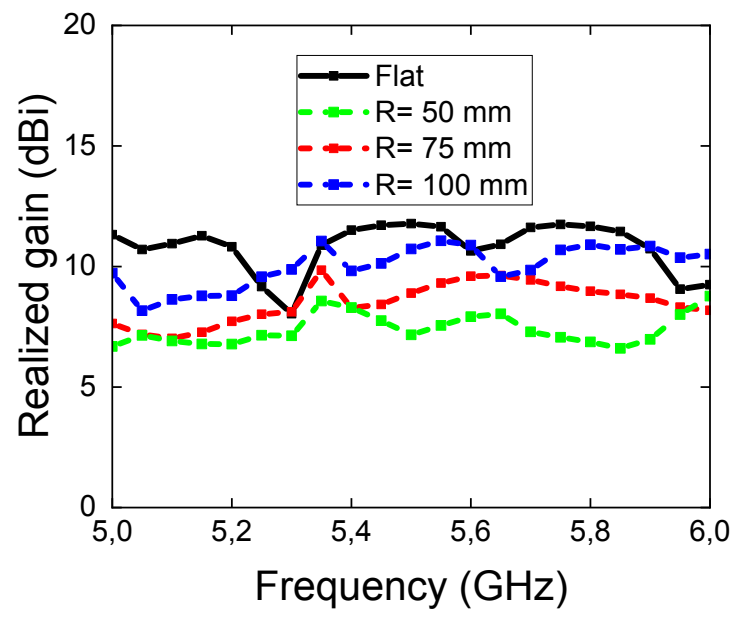

(b)

Figure 5.11: (a) Gain of MSA at different curvatures. (b) Gain of MPA array at different curvatures. 
The efficiency of both designs is also studied at different curvatures. Figure 5.12a shows a slight variation in the efficiency of MSA with different curvatures, whereas, the MPA array shows dramatic variation in the efficiency as depicted in Fig. 5.12b. Due to changing the curvature, the efficiency of MPA array is dramatically deteriorated around the operating frequency of $5.8 \mathrm{GHz}$, and strongly enhanced around $5.3 \mathrm{GHz}$.

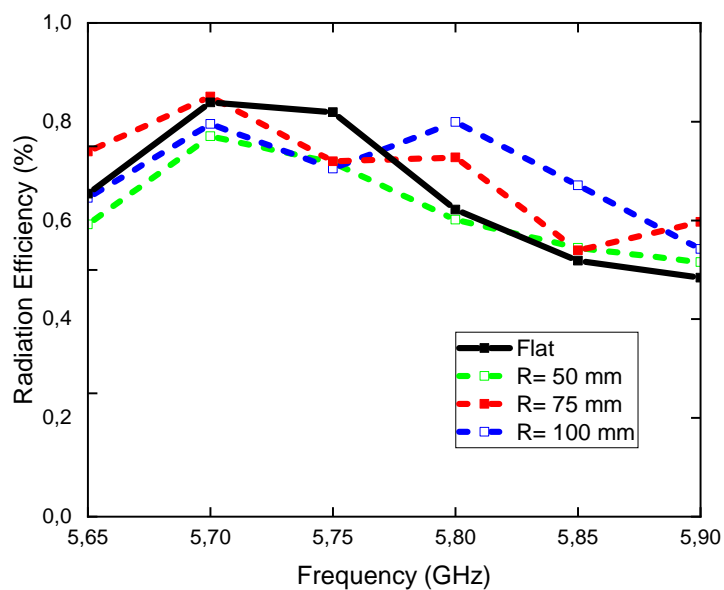

(a)

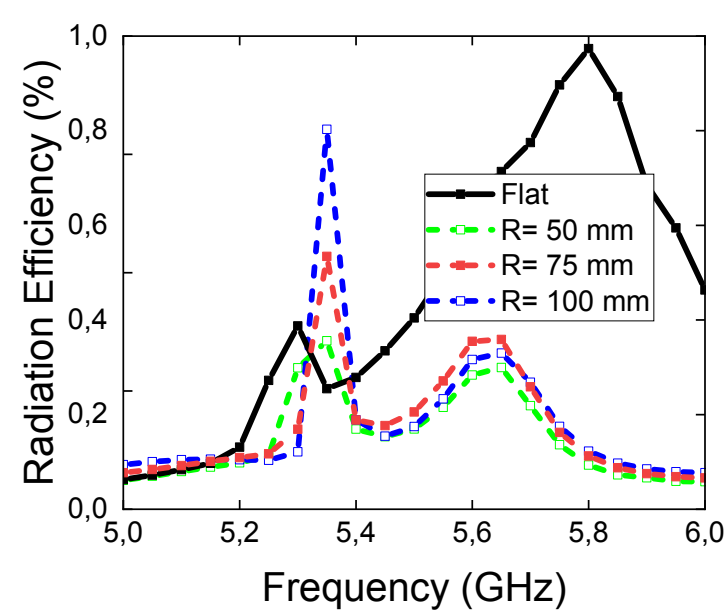

(b)

Figure 5.12: (a) Efficiency of MSA at different curvatures. (b) Efficiency of MPA array at different curvatures.

\subsection{Rectenna System Utilizing MSA}

The main components of a generic rectenna system are illustrated in Figure 5.13. The rectenna system can be utilized in a point-to-point power transmission link, where the rectenna's design limitations can be adjusted to the characteristics of the largely known (or predicted) incident field, such as its frequency, polarisation, and power density (see [74] as an example), or can be used to harvest ambient EM radiation over specific frequency band (see $[75,76]$ as an example). In the latter application, where the rectenna acts as an energy scavenger, since the incoming radiation is highly unpredictable in terms of frequency bandwidth, polarization and power density, the design of the rectenna can be challenging.

Harvesting the ambient EM radiation was investigated in urban and semi-urban areas. For instance, in [75], it was demonstrated that an ambient EM wave with power density 
of $-25 \mathrm{dBm} / \mathrm{cm}^{2}$ can be harvested with $40 \%$ efficiency using a single band rectenna, and an ambient wave with power density of $-29 \mathrm{dBm} / \mathrm{cm}^{2}$ can be harvested with a multi-band rectenna system. To evaluate the efficiency of a system with fluctuating levels of available power, in [75], the efficiency was calculated using energy instead of power.

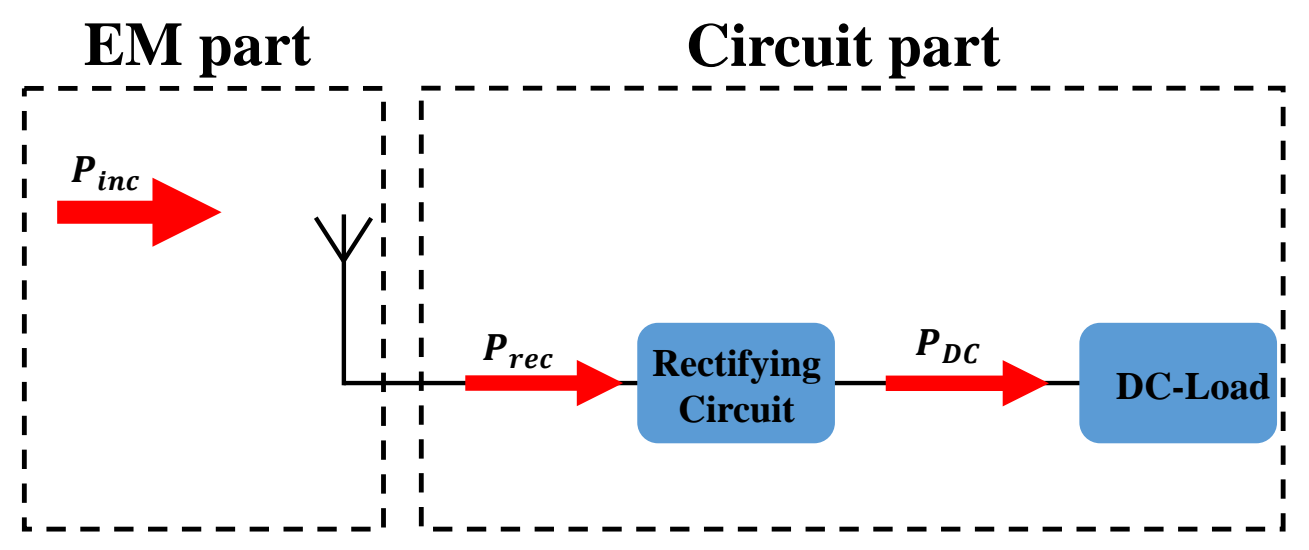

Figure 5.13: Basic components of a generic rectenna system comprising a receiving antenna, a rectification circuit (may include matching circuits on both ends) and a DC load.

\subsubsection{Rectenna Design}

I present an entire rectenna system in this work, with the suggested MSA serving as the EM component. To design the rectifying circuit, I connected the Thevenin equivalent circuit of the MSA with the circuit shown in Figure 5.14. In ADS, I utilized the DAC component to control the values of each component in the equivalent circuit. To account for the circuit's non-linearity, the harmonic balance controller was used in our simulation with the first five harmonics. Although increasing the harmonic order improves accuracy, the power content after the fifth harmonic is negligible. In fact, I conducted the simulation at a higher order to assess the effect of increasing the order of harmonics, and the results were the same.

As illustrated in Fig.5.14, the circuit consists of HSMS-2860 Schottcky diode [103], 11 segments of transmission lines printed on the top of a $1.27 \mathrm{~mm}$ thick Roger 3006 dielectric substrate having a loss tangent of $\tan \delta=0.0027$ and a dielectric constant of $\epsilon_{r}=$ 6.15 , and a DC load. To achieve maximum output DC power, the widths of transmission lines' segments were fixed to $1.8 \mathrm{~mm}$. The lengths of segments and the DC load were optimized using the gradient optimization feature in ADS. The lengths of the segments of the optimized circuit and the optimum load are shown in Fig. 5.14. 


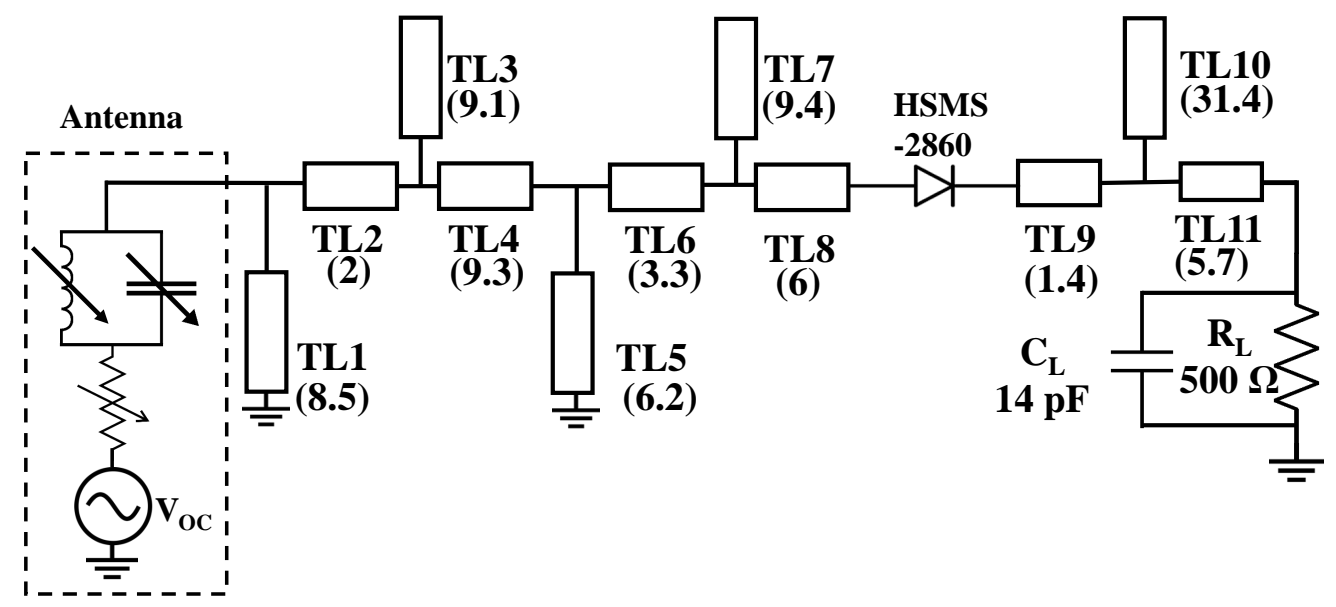

Figure 5.14: Schematic diagram of the rectifying circuit with the incorporated equivalent circuit of the MSA.

It is important to note that the optimized circuit was based on an incident field with specific characteristics in terms of polarization, incident angle and power density. For wireless power transfer applications where the incident field characteristics are known, the optimized rectification circuit just described will deliver maximum power to the optimized load. However, for energy harvesting of incoming radiation without any known characteristics (aside from the frequency range), then the field-specific optimized rectification circuit may not be optimal over the possible variance in the characteristics of the incident field.

Using the time-domain solver in CST, the antenna was illuminated by a linearlypolarized plane wave. The power density of the plane wave was $10 \mathrm{~W} / \mathrm{m}^{2}$ and the angle of incidence $(\theta)$ was zero degree (i.e., the $\mathrm{k}$ vector is coming from the positive $\mathrm{z}$-direction. Then, $V_{o c}$ was extracted from the full-wave simulation of the TPA (when the antenna's terminals were open-circuited).

The extracted parameters are processed and loaded to the equivalent circuit of the antenna shown in Fig. 5.14. Using ADS, the received AC power, $P_{\text {rec }}$, and the output DC power, $P_{d c}$, of the rectenna system were obtained (ref to Fig.5.14). Figure 5.15 shows the simulation results of $P_{r e c}$ and $P_{d c}$. As illustrated in Fig.5.15, the maximum Ac power is approximately $7.8 \mathrm{~mW}$, and the maximum DC power is $6.3 \mathrm{~mW}$. Figure 5.16 depicts the variation of the efficiency of the rectifying circuit with frequency. The maximum efficiency of the rectifying circuit is obtained at $5.8 \mathrm{GHz}$ with an efficiency of $81 \%$. 


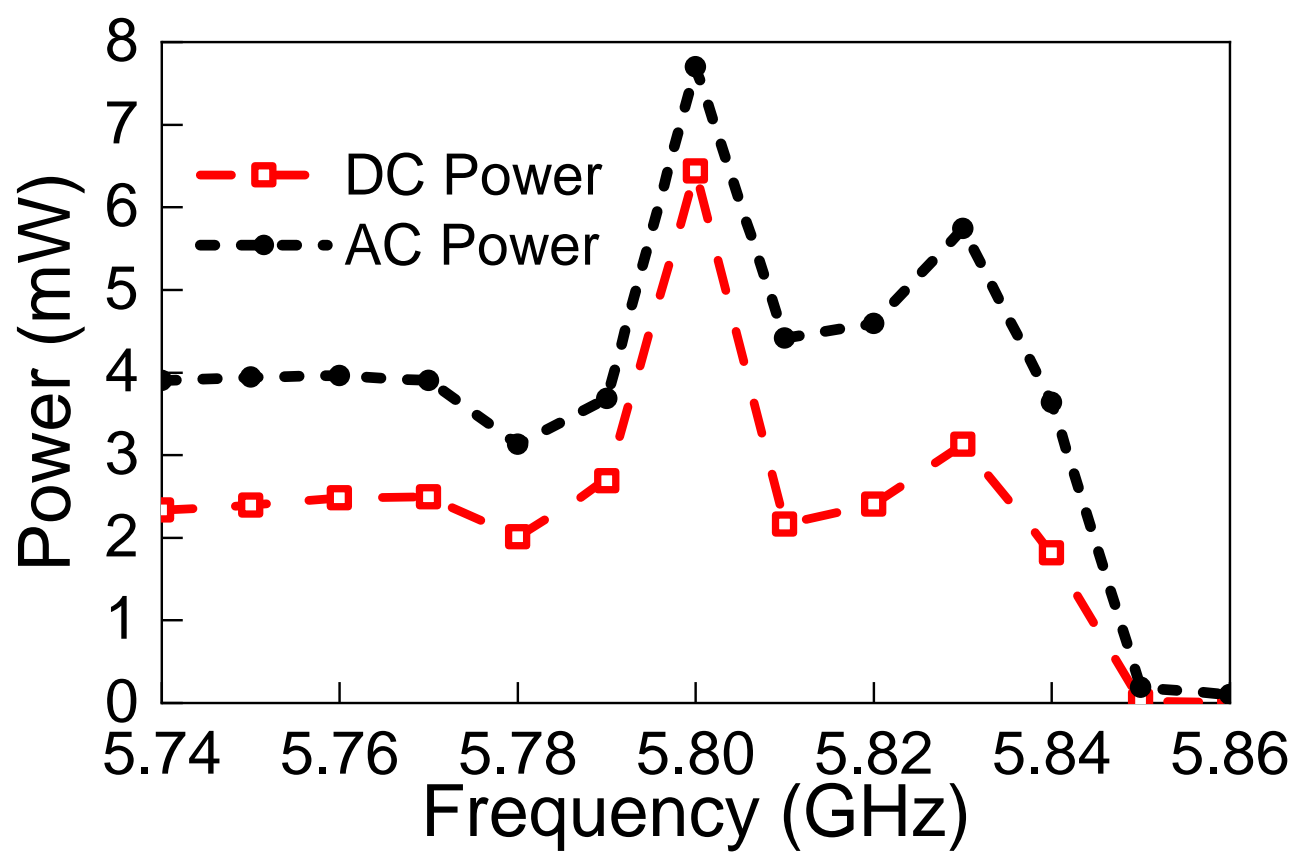

Figure 5.15: Simulated input and output power of the rectifying circuit in $\mathrm{mW}$.

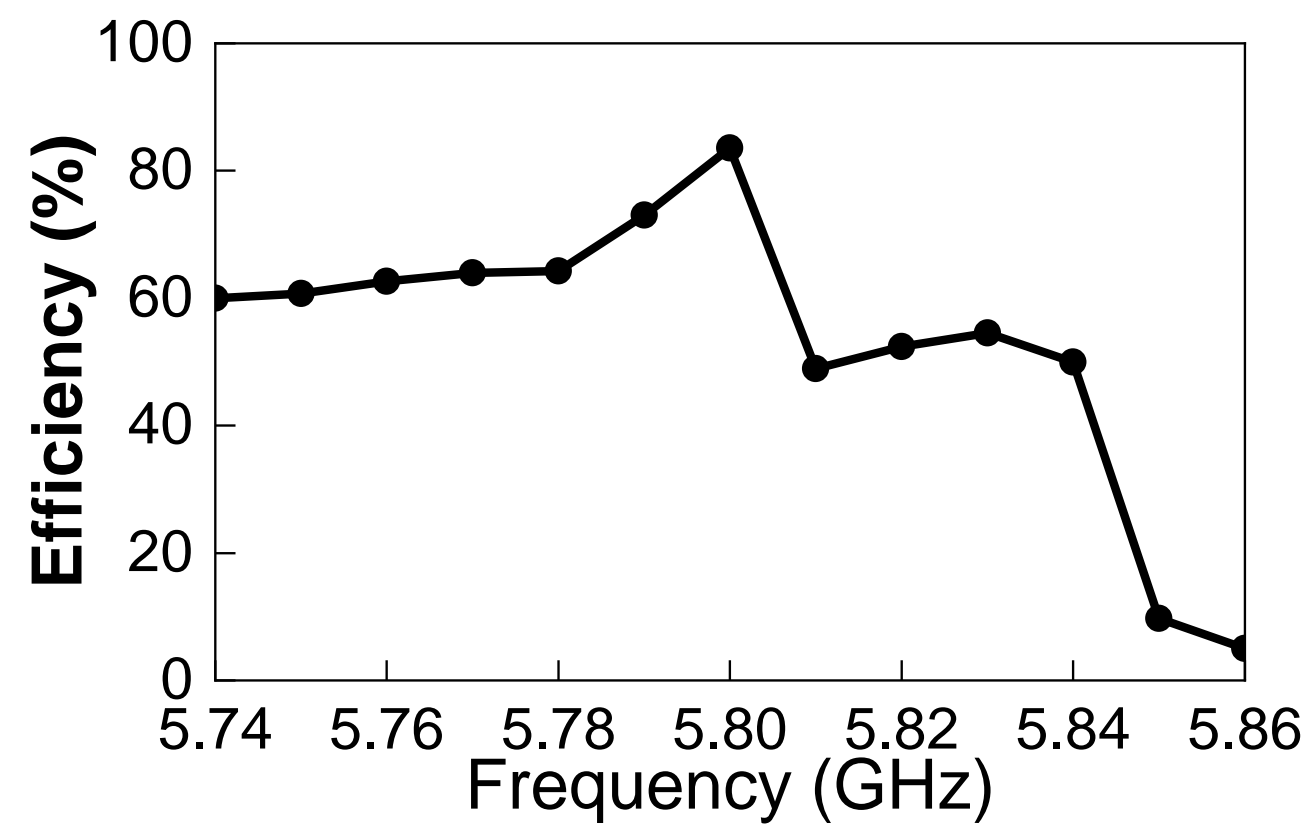

Figure 5.16: Power efficiency $\left(\eta_{a c-d c}\right)$ of the rectifying circuit. 


\subsection{MSA Operating in Fifth Generation Band}

The extensive research into fifth-generation $(5 \mathrm{G})$ technology is a strong indication of a technological revolution to satisfy the ever-increasing demand and needs for high-speed connectivity and Internet of Things (IoT)-based applications. The design described in 5.1 is optimized for two different bands, namely Wi-Fi band (around $5.8 \mathrm{GHz}$ ) and $5 \mathrm{G}$ band (around $26 \mathrm{GHz}$ ). The performance of the design for Wi-Fi band were studied in the previous sections.

In this section, the characteristics of the MSA in $5 \mathrm{G}$ band is analyzed. The reflection coefficient of the design was numerically simulated and measured around $26 \mathrm{GHz}$. Figure 5.17 illustrates the simulated and the measured reflection coefficient of the proposed design. The design resonates in the frequency range of $25.5 \mathrm{GHz}$ to $26.1 \mathrm{GHz}$, according to numerical simulations. For frequencies ranging from $25.46 \mathrm{GHz}$ to $26.68 \mathrm{GHz}$, the measured reflection coefficient is less than $-10 \mathrm{~dB}$. It's worth noting that the measuring results shows a wider band than the simulation findings, which could be due to port soldering.

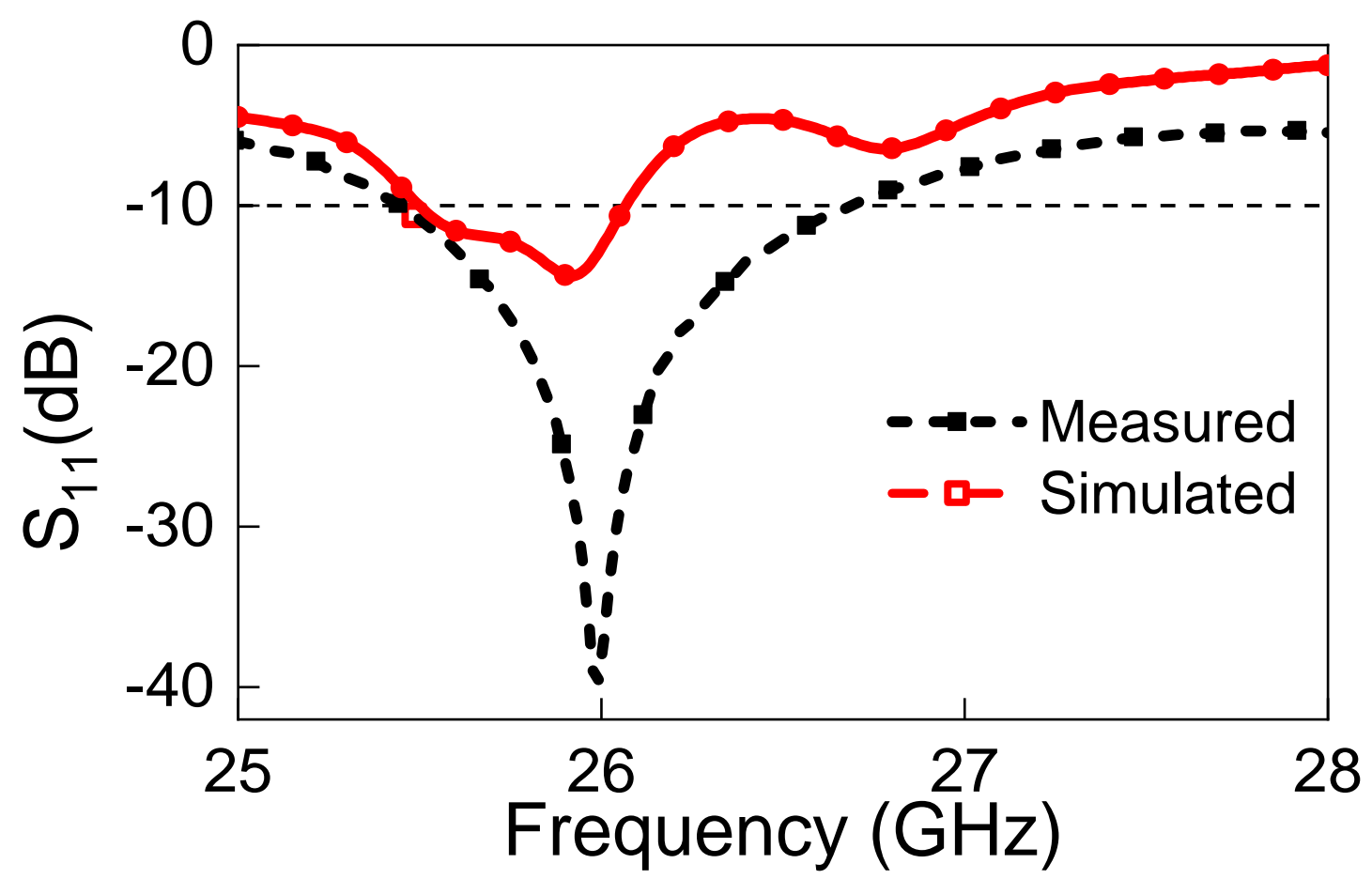

Figure 5.17: Simulated and measured reflection coefficients of the proposed MSA around $26 \mathrm{GHz}$. 
The gain and the total efficiency of the MSA were numerically investigated around 26 GHz. Observing Figure 5.18a, the gain of the design is ranging from $9 \mathrm{~dB}$ to $12 \mathrm{~dB}$ over the studied spectrum with a maximum value at $26 \mathrm{GHz}$. Figure $5.18 \mathrm{~b}$ depicts the total efficiency of the design for frequency ranging from $25 \mathrm{GHz}$ to $28 \mathrm{GHz}$. The efficiency is fluctuating between 0.3 and 0.95 with a maximum efficiency at $26 \mathrm{GHz}$ as well.

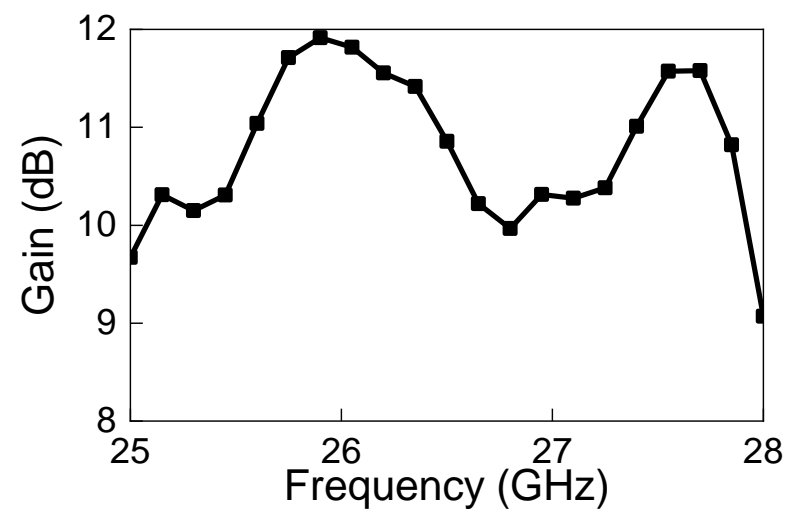

(a)

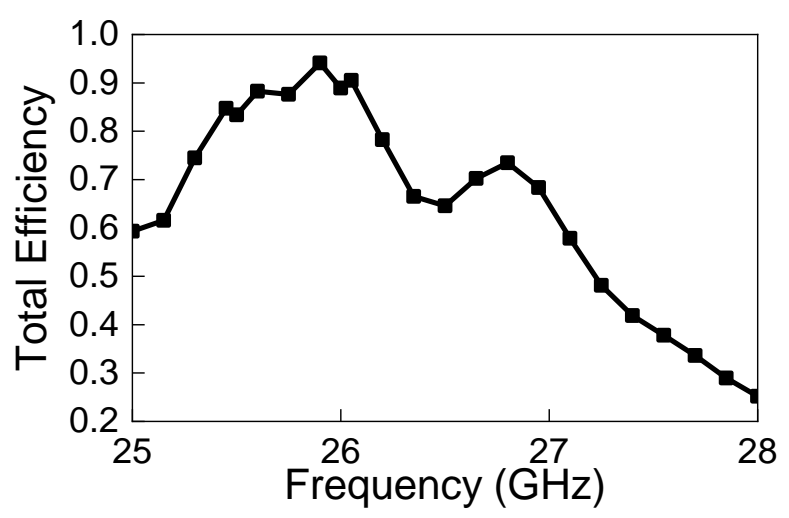

(b)

Figure 5.18: (a) Gain of MSA around $26 \mathrm{GHz}$. (b) Total efficiency of MSA around $26 \mathrm{GHz}$.

\subsection{Conclusion}

In this chapter, I presented an MSA that operates around $5.8 \mathrm{GHz}$ with a feeding network to channel all the received signals into one port. Additionally, I utilized same material and footprint to design an MPA array that operates at the same frequency. To compare their performances, both designs are studied under flat and bent states. Comparison results demonstrated that the MSA provides stable realized gain and efficiency compared to MPA array during the conformal operation. Additionally, in the case of the $\mathrm{x}$-axis bending deformation, the resonant frequency of MSA is slightly shifted for all selected values, whereas the resonant frequency of MPA array is appreciably changed during the bending scenarios.

Since all the comparison results illustrate that the MSA is more stable at various curvatures in the ISM band, a rectenna system utilizing the proposed MSA was developed to operate around $5.8 \mathrm{GHz}$. The suggested rectenna optimized to provide high AC-to- 
DC conversion efficiency. An AC-to-DC conversion efficiency of approximately $81 \%$ was obtained at $5.8 \mathrm{GHz}$.

The capability of the proposed MSA to operate at higher frequency (around $26 \mathrm{GHz}$ ) was investigated. The numerical simulation result shows that the design resonates from $25.5 \mathrm{GHz}$ to $26.1 \mathrm{GHz}$. Wider band was obtained by measurement ranging from $5.46 \mathrm{GHz}$ to $26.68 \mathrm{GHz}$. Also, I numerically studied the gain and the efficiency of the design around $26 \mathrm{GHz}$. The MSA showed a maximum gain of $12 \mathrm{~dB}$ and a maximum efficiency of 0.95 at $26 \mathrm{GHz}$. 


\section{Chapter 6}

\section{Adjustable Input Impedance Dipole For Rectenna System}

\subsection{Introduction}

Inspired by the availability of electromagnetic energy, the idea of energy harvesting, using rectennas, was originally proposed by Brown [123]. Since then, a significant amount of research has been conducted to investigate different types of rectennas, particularly for their energy conversion efficiency. Figure 6.1 shows the main components of a rectenna system. As illustrated in the figure, it consists of an antenna, rectifying circuit (which typically includes matching circuits and a rectifier circuit), and a load [124, 89, 125]. To ensure maximum power transfer from the antenna to the rectifiying circuit, the antenna needs to be cascaded with a matching circuit $[110,126]$. Besides the complexity added to the system, the use of a matching circuit increases power loss since Ohmic losses can be appreciable in real-world capacitors and inductors. In their work Almoneef et al., [127] proposed a folded dipole and a pair of capacitors in a stacked rectenna configuration to match the antenna to a diode directly. In this work, I present a simple design of a dipole antenna with adjustable input impedance such that the antenna can be directly matched to the input impedance of diode circuits. The rest of the paper is structured as follows: Section 2 discusses the geometry of the proposed design along with the obtained simulation results. It also highlights the input impedance and the resonant frequency analysis of the proposed design. Section 3 investigates the effect of varying the dimensions of the design on the input impedance. Sections 4 illustrates the capability of using the proposed design in a rectenna system and presents a comparison between the conversion efficiency of the 
rectenna system that uses our design and one with a matching circuit. Section 5 concludes with a discussion.

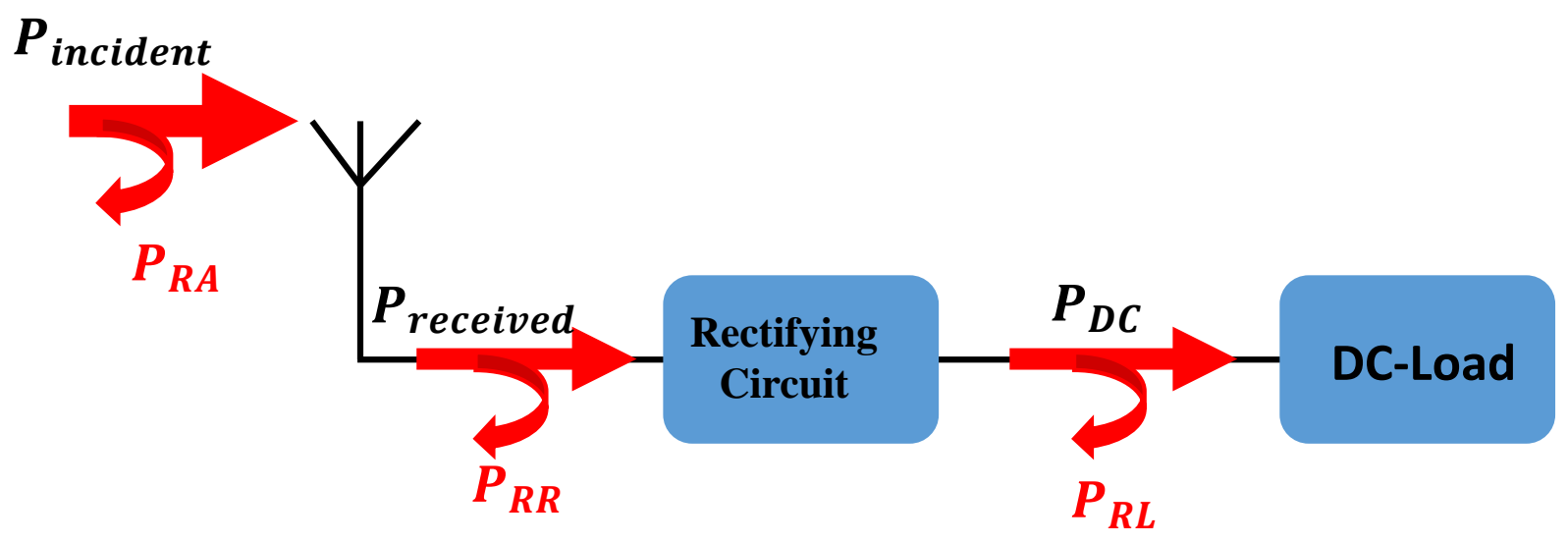

Figure 6.1: Rectenna system components.

\subsection{Design Methodology}

In Figure 6.1, $P_{\text {incident }}$ represents the total incoming power from an isolated electromagnetic radiation sources; $P_{\text {received }}$ is the power delivered to the diode; $P_{R A}, P_{R R}$, and $P_{R L}$ are the reflected power components at the front end of antenna, rectifying circuit, and the DC load, respectively; $P_{D C}$ is the output DC power. To enhance the conversion efficiency of the rectenna system, ideally, the incoming power $\left(P_{\text {incident }}\right)$ should be equal to the power delivered to the diode $\left(P_{\text {received }}\right)$. This can be achieved by either designing an efficient matching circuit or designing an antenna that has a complex conjugate of the input impedance of the rectifying circuit (since the impedance of the diode is fixed). The input impedance of a diode can be obtained from the diode model provided by the manufacturer or obtained from measurements [128]. As an example, the input impedance of the HSMS2860-Schottcky diode is $Z_{d}=187-\mathrm{j} 39 \Omega$ when terminated by a $300 \Omega$ resistive load $[127,128]$. To avoid designing a matching circuit, which, in practice, increases the power loss in the rectenna system due to Ohmic losses in inductors and capacitors, an adjustable input impedance antenna is highly desired.

Figure 6.2 shows the configuration of the proposed dipole design. The dipole consists of two parts: the first part is a dipole with a length of $L_{1}$, while the second part is the two controlling arms that are attached at the edge of the gap endpoints. By adjusting 
the length of these controlling arms $\left(L_{2}\right)$, one can tune the reactance part of the input impedance, whereas the real part of the impedance can be tuned by varying the width of these arms $W_{1}$. I used Roger material (RO4003C) with $\epsilon_{r}=3.38$ and thickness $\mathrm{h}=1.52 \mathrm{~mm}$. The fabricated design has $L_{1}=23.3 \mathrm{~mm}, L_{2}=6 \mathrm{~mm}, \mathrm{~g}=1 \mathrm{~mm}$ and $W_{1}=W_{2}=2 \mathrm{~mm}$. Using the full-wave simulator (Ansys HFSS), the antenna provides an input impedance of $Z_{i n}=$ $187+\mathrm{j} 39 \Omega$ at $3 \mathrm{GHz}$ as depicted in Figure 6.3. Adjusting the port's impedance to $187+\mathrm{j} 39$ $\Omega$, I simulated the reflection coefficient of the design. Figure 6.4 shows good resonant at 3 $\mathrm{GHz}$

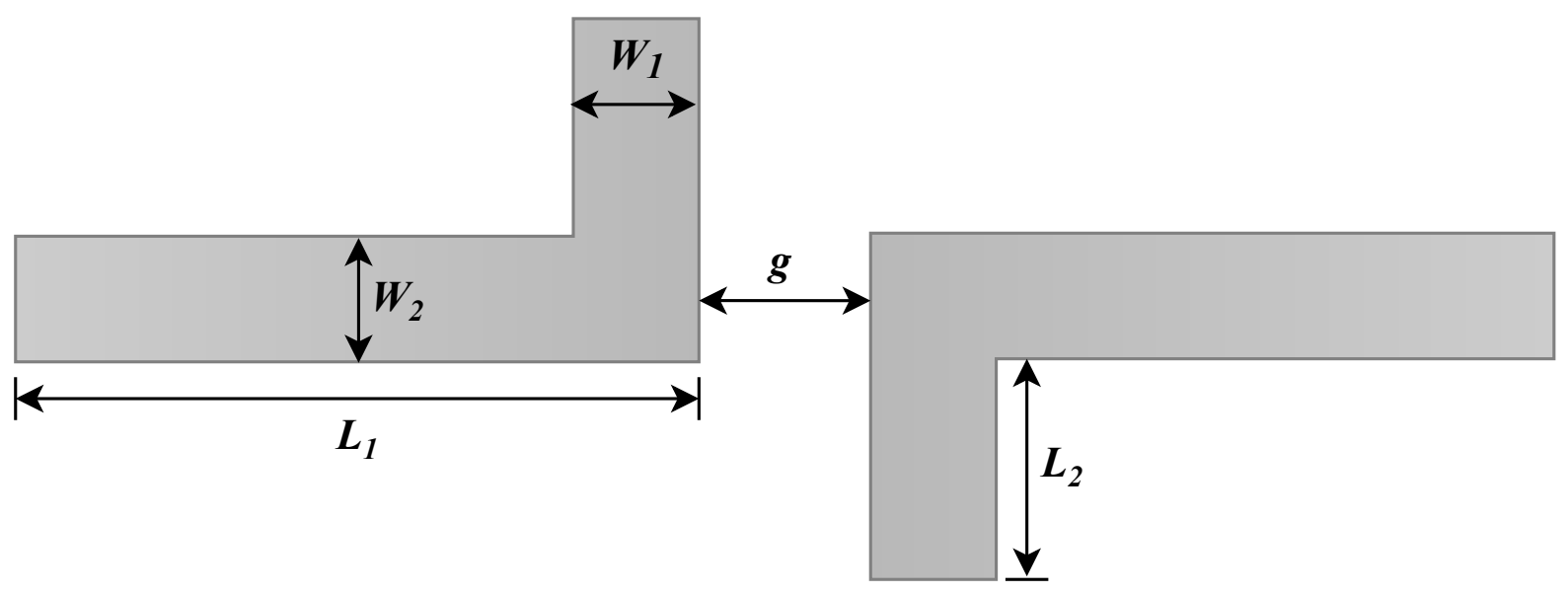

Figure 6.2: Schematic showing the antenna design and its parameters.

\subsection{Parametric Study of the Proposed Design}

A parametric study has been conducted to verify the validity of the proposed design. As for the first step towards studying the effect of the controlling arms' length on the input impedance, I fixed all the dimensions of the design and simulated it at different length values. Figure 6.5 shows the real part of the simulated input impedance of the proposed design of different arms' lengths $\left(L_{2}\right)$, whereas, Figure 6.6 depicts the imaginary part. Table 6.1 illustrates that as the length of the controlling arms increases, the dipole shows slight variations in the real part of the input impedance, whereas the imaginary part is more sensitive to these length variations. Moreover, the design shows a capacitive input impedance at $L_{2}=10 \mathrm{~mm}$. 


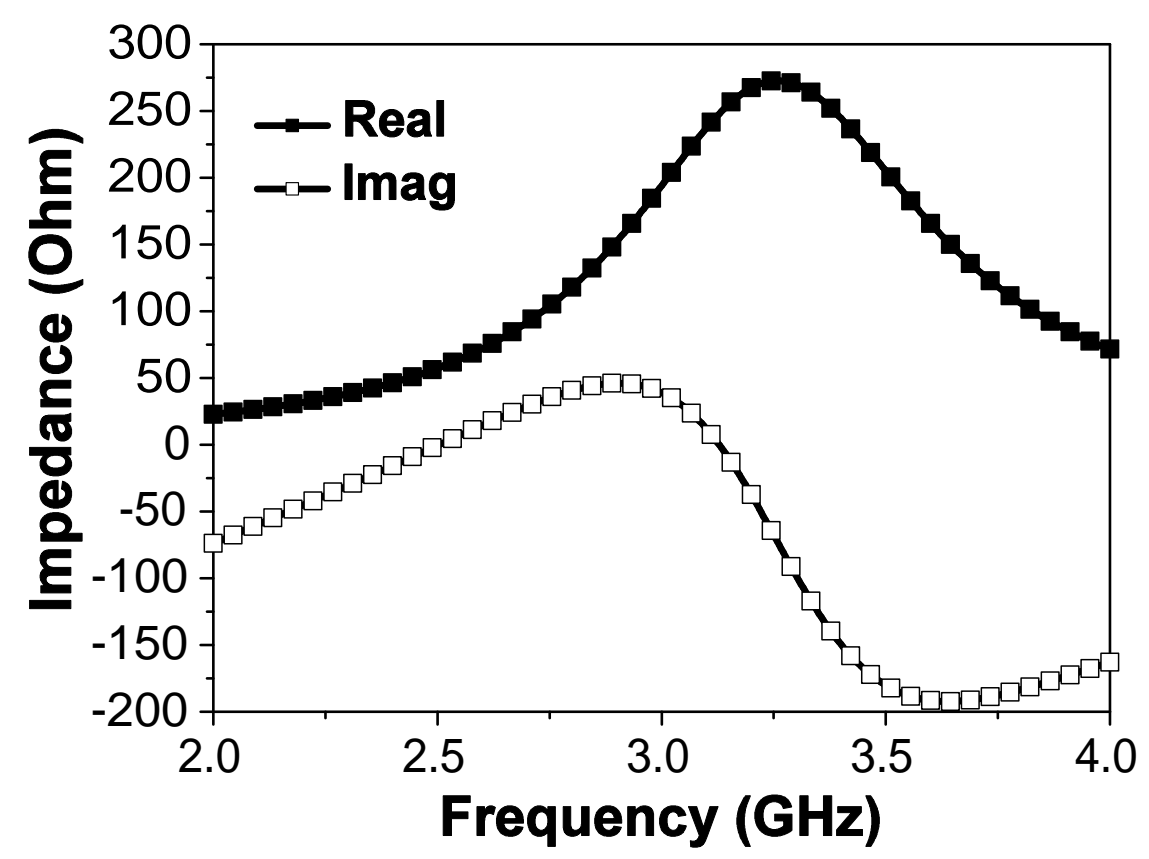

Figure 6.3: Simulated input impedance of the proposed antenna. The input impedance is $187+\mathrm{j} 39 \Omega$ at $3 \mathrm{GHz}$.

To study the effect of the width on the input impedance, I fixed all dimensions and changed the width of the controlling arms $\left(W_{2}\right)$, then I fixed $W_{2}$ and changed $W_{1}$. Table 6.2 exhibits the variations of the input impedance due to different $W_{2}$. As shown in the table, both the real and the imaginary parts are decreasing with the increasing width $\left(W_{2}\right)$. On the other hand, Table 6.3 shows the effect of changing $W_{1}$ on the input impedance. It can be noted that both the real and the imaginary parts are decreasing with increasing $W_{1}$. However, the input impedance of the design is more sensitive to variations in $W_{1}$ than to variations in $W_{2}$.

Table 6.4 shows the effect of the gap on the input impedance. As can be seen, the real part slightly decreases when the gap increases while the imaginary part increases proportionally. 


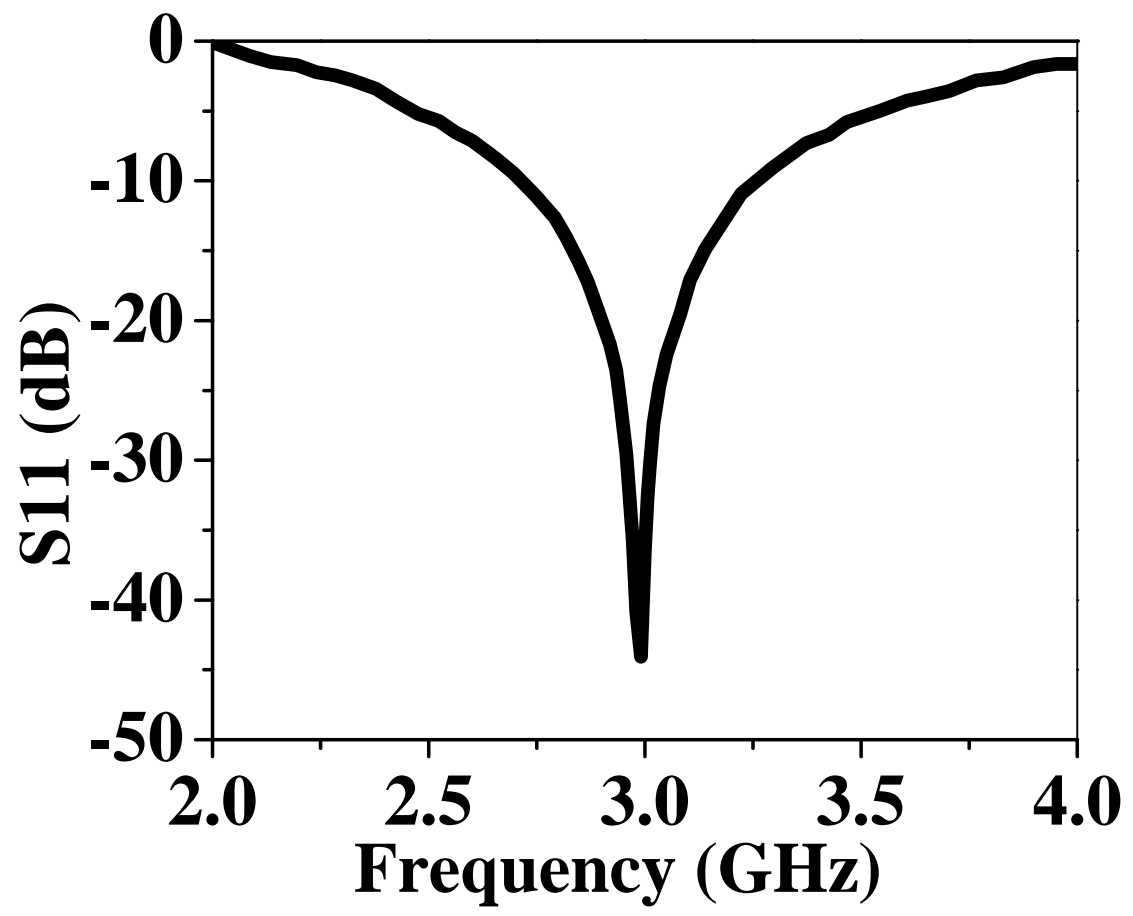

Figure 6.4: Simulated reflection coefficient using port of $187+\mathrm{j} 39 \Omega$ reference impedance.

Table 6.1: Simulated input impedance of the proposed design for different $L_{2}$. Other antenna parameters were fixed at $L_{1}=23.3 \mathrm{~mm}, W_{1}=W_{2}=2 \mathrm{~mm}$ and $\mathrm{g}=1 \mathrm{~mm}$.

\begin{tabular}{||c|c|c|c|c|c||}
\hline$L_{2}(\mathrm{~mm})$ & 2 & 4 & 6 & 8 & 10 \\
\hline Real $(\Omega)$ & 174.32 & 186.92 & 187 & 192.79 & 173.58 \\
\hline Imaginary $(\Omega)$ & 85.91 & 64.58 & 39 & 10.77 & -24.3 \\
\hline
\end{tabular}

Table 6.2: Simulated input impedance of the proposed design for different $W_{2}$. Other antenna parameters were fixed at $L_{1}=23.3 \mathrm{~mm}, L_{2}=6 \mathrm{~mm}, W_{1}=2 \mathrm{~mm}$ and $\mathrm{g}=1 \mathrm{~mm}$.

\begin{tabular}{||c|c|c|c|c|c||}
\hline$W_{2}(\mathrm{~mm})$ & 1 & 2 & 3 & 4 & 5 \\
\hline Real $(\Omega)$ & 202.59 & 187 & 180.33 & 166.24 & 152.75 \\
\hline Imaginary $(\Omega)$ & 49.21 & 39 & 36.53 & 28.58 & 26.68 \\
\hline
\end{tabular}




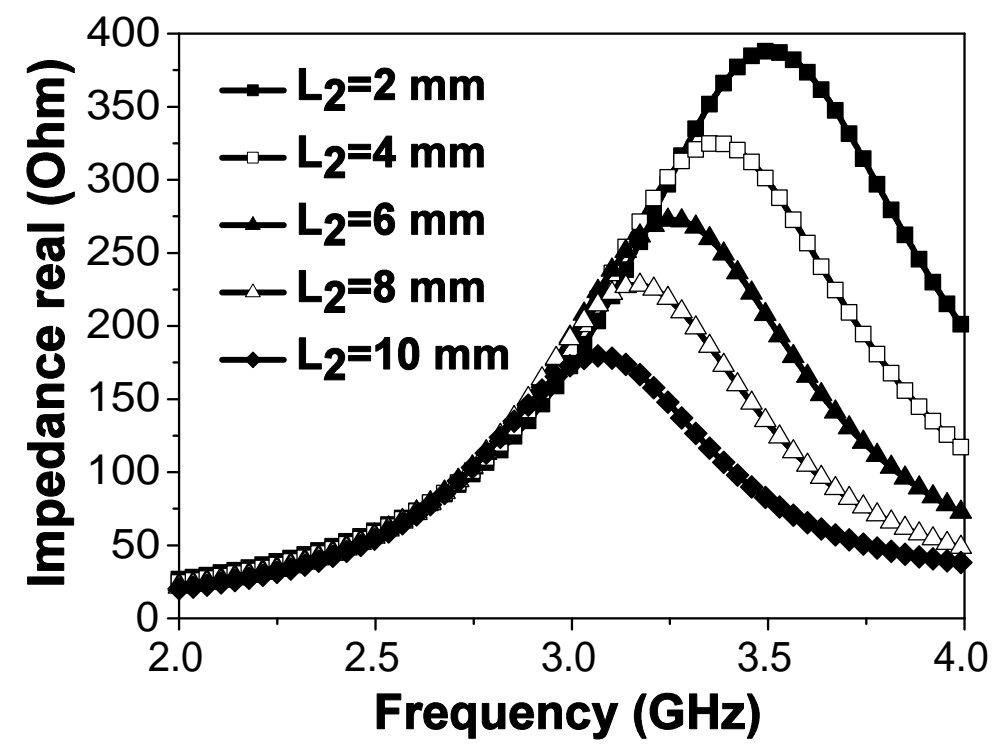

Figure 6.5: Real part of the simulated input impedance of the proposed design for different $L_{2}$.

Table 6.3: Simulated input impedance of the proposed design for different $W_{1}$. Other antenna parameters were fixed at $L_{1}=23.3 \mathrm{~mm}, L_{2}=6 \mathrm{~mm}, W_{2}=2 \mathrm{~mm}$ and $\mathrm{g}=1 \mathrm{~mm}$.

\begin{tabular}{||c|c|c|c|c|c||}
\hline$W_{1}(\mathrm{~mm})$ & 1 & 2 & 3 & 4 & 5 \\
\hline Real $(\Omega)$ & 245.6 & 187 & 169.28 & 147.27 & 133.4 \\
\hline Imaginary $(\Omega)$ & 78.49 & 39 & 9.47 & -6.54 & -18.58 \\
\hline
\end{tabular}

Table 6.4: Simulated input impedance of the proposed design for various gaps (g). Other dimensions were fixed at $L_{1}=23.3 \mathrm{~mm}, L_{2}=6 \mathrm{~mm}$ and $W_{2}=W_{1}=2 \mathrm{~mm}$.

\begin{tabular}{||c|c|c|c||}
\hline $\mathrm{g}(\mathrm{mm})$ & 1 & 2 & 3 \\
\hline Real $(\Omega)$ & 187 & 174.69 & 160.27 \\
\hline Imaginary $(\Omega)$ & 39 & 63.85 & 75.65 \\
\hline
\end{tabular}




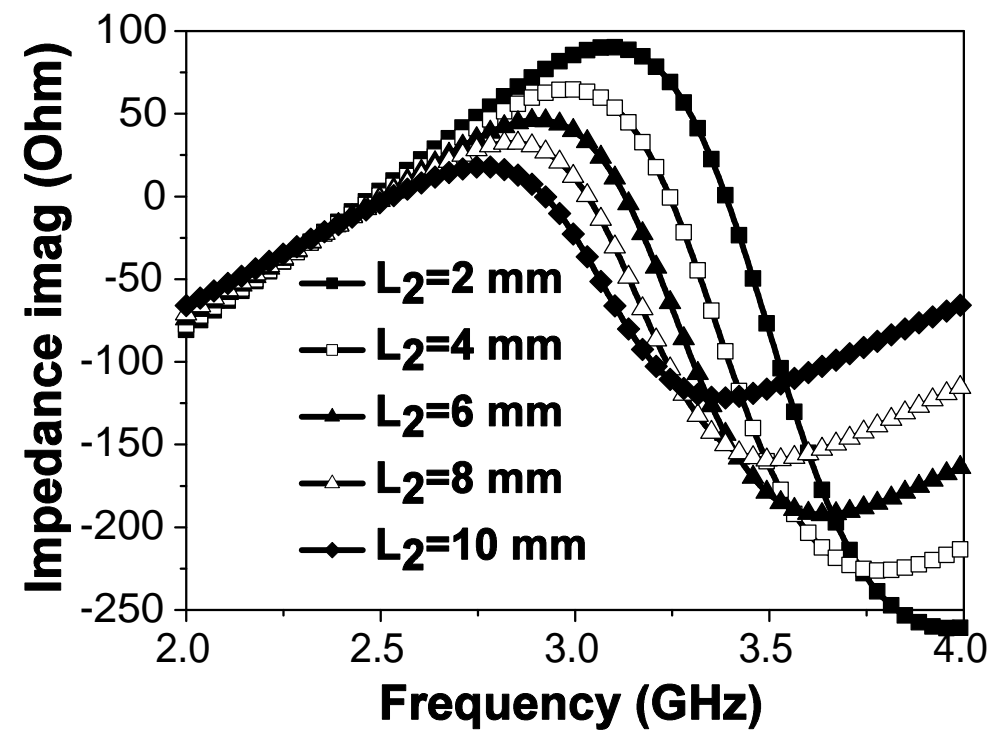

Figure 6.6: Imaginary part of the simulated input impedance of the proposed design for different $L_{2}$. 


\subsection{Implementation in a Rectenna's Design}

To verify the applicability of the impedance-tuned antenna, I designed two rectenna systems, one utilizing the proposed design without a matching circuit and the other design using a conventional antenna and a matching circuit. Utilizing the impedance-tuned, the antenna can be connected directly to the rectifying circuit without using a matching circuit as depicted in Fig. 6.7. As shown in the figure, the circuit is composed of a diode, a transmission line, an open circuit stub and an RL circuit. The transmission line and the open circuit stub were used to suppress the higher harmonics of the rectified wave before reaching the load. The parameters of the transmission lines and open stub are depicted in Table 6.5. A shunt capacitor of $40 \mathrm{pF}$ was used to filter out the high-frequency harmonics. In this design, I used HSMS-2860-Schottky diode because of its low turn-on voltage and its relatively high-speed switching. To obtain maximum conversion efficiency, I carried out the optimization in ADS to determine the optimum input impedance [104]. The maximum conversion efficiency was obtained at an input impedance of $Z_{i n}=159.5+\mathrm{j} 12 \Omega$ and a frequency of $2 \mathrm{GHz}$. Utilizing full-wave simulator, I designed an impedance-tuned dipole with the following parameters: $L_{1}=35.5 \mathrm{~mm}, L_{2}=13 \mathrm{~mm}, \mathrm{~g}=1 \mathrm{~mm}, W_{1}=2$ and $W_{2}=2.5$ mm. In this design, I used Roger material (RO4003C) with $\epsilon_{r}=3.38$ and thickness $\mathrm{h}=1.52$ $\mathrm{mm}$. Figure 6.8 shows the simulated input impedance of the proposed design. To mimic the behavior of the dipole, an one-tone frequency power source was used in the simulation. The $\mathrm{Z}$ parameters of the dipole were extracted and imported into ADS. Those parameters were stored in Data Access Component (DAC) and were assigned as the input impedance of the source (see Fig. 6.7). DAC enables us to vary the input impedance of the source at each point of frequency according to the variation of the input impedance of the antenna with frequency. The power of the source was fixed at $0 \mathrm{dBm}$.

Table 6.5: Parameters of the transmission lines segments of the matching circuit in Fig. 6.7.

\begin{tabular}{|c|c|c|}
\hline TLn & Length $\mathbf{( m m )}$ & Width $\mathbf{~} \mathbf{m m})$ \\
\hline TL1 & 21 & 0.75 \\
\hline TL2 & 1.6 & 1.9 \\
\hline TL3 & 3.4 & 1.9 \\
\hline
\end{tabular}

Figure 6.9 shows a rectenna system using a matching circuit between the source and the diode. In this design, I replaced the adjustable antenna with a conventional antenna of 50 $\Omega$ input impedance and a matching circuit. The matching circuit consists of a transmission 


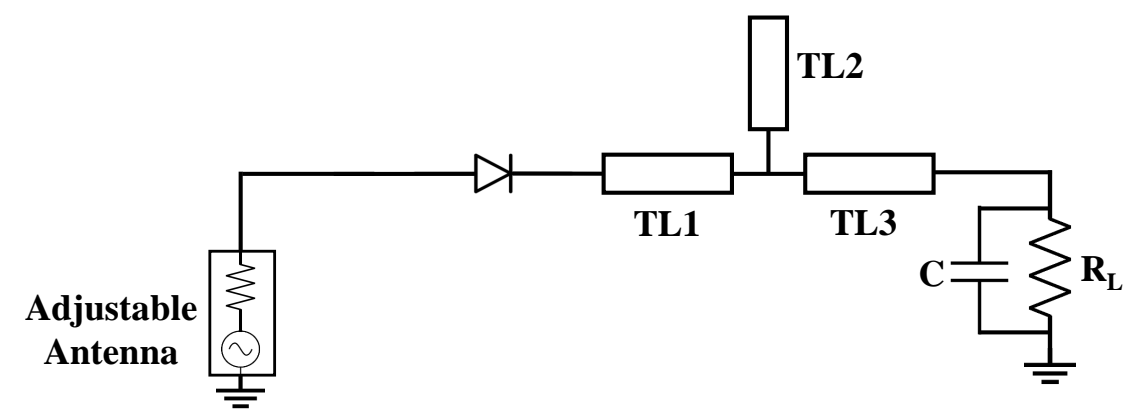

Figure 6.7: A schematic showing the rectifier circuit designed in the ADS simulator. The transmission lines parameters are listed in Table 6.5. The circuit is terminated by a parallel combination of load resistor of a $4.5 \mathrm{~K} \Omega$ and a $40 \mathrm{pF}$ capacitor.

line, shorted and opened circuit stubs. This circuit was optimized to obtain the maximum conversion efficiency. The dimensions of the transmission lines of the optimized circuit are listed in Table 6.6. Additionally, a rectenna system with a $50 \Omega$ input impedance antenna and without a matching circuit was designed and simulated.

Table 6.6: Parameters of the transmission lines segments of the rectenna system in Fig. 6.9 .

\begin{tabular}{|c|c|c|}
\hline TLn & Length $\mathbf{( m m )}$ & Width $\mathbf{( m m )}$ \\
\hline TL1 & 0.75 & 6.9 \\
\hline TL2 & 6.60 & 0.5 \\
\hline TL3 & 8.20 & 1.2 \\
\hline TL4 & 21 & 0.75 \\
\hline TL5 & 1.6 & 1.9 \\
\hline TL6 & 3.4 & 1.9 \\
\hline
\end{tabular}

To determine the most optimal circuit, a comparision is made between the conversion efficiencies of all cases considered. The rectenna system's conversion efficiency is defined as the ratio between the output DC power, $\left(P_{D C}\right)$, and the received power, $\left(P_{\text {received }}\right)$ (see Fig. 6.1) [38, 84, 61]:

$$
\eta=\frac{P_{D C}}{P_{\text {incident }}} \times 100 \%=\frac{V_{D C}^{2} / R_{L}}{P_{\text {incident }}} \times 100 \%
$$

In all designs, I fixed the received power at $0 \mathrm{dBm}$. As illustrated in Fig. 6.10, both the 


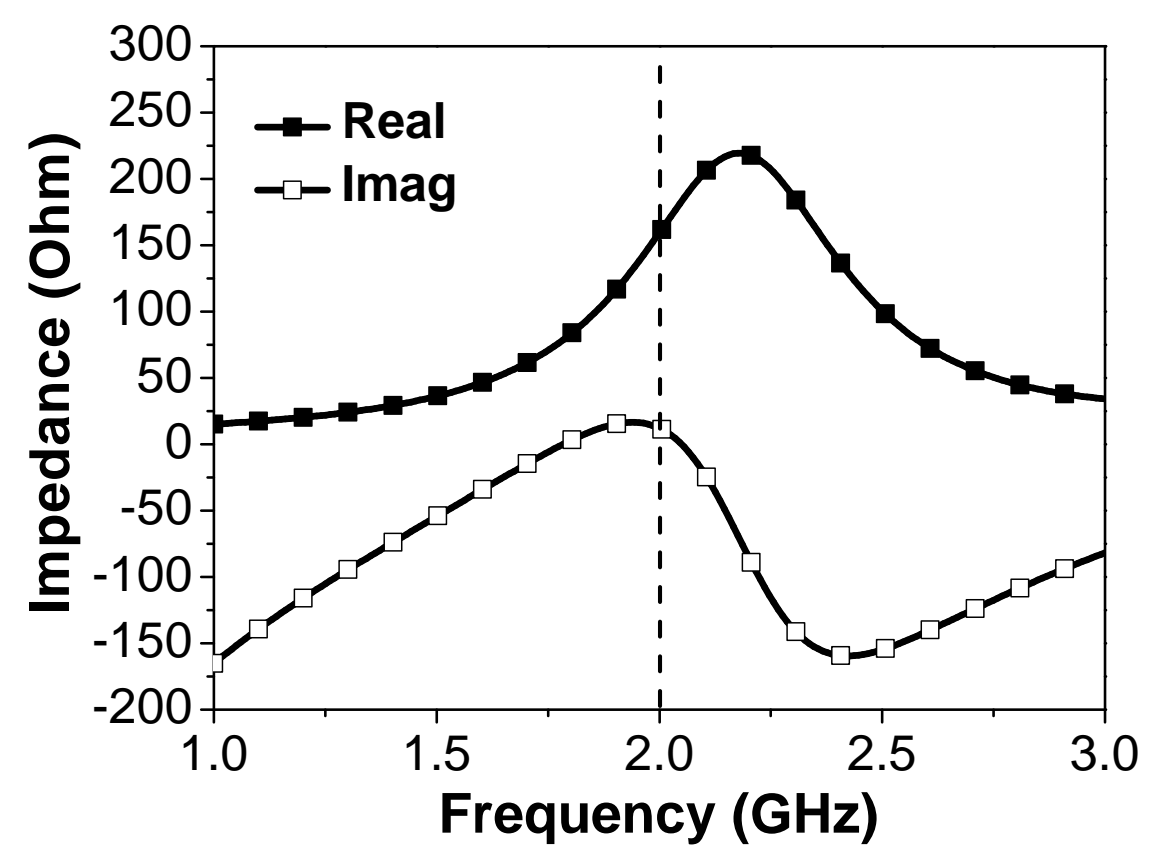

Figure 6.8: Simulated input impedance of the proposed antenna. The input impedance is $159.5+\mathrm{j} 12 \Omega$ at $2 \mathrm{GHz}$.

adjustable antenna and the matching circuit show a higher conversion efficiency compared with the circuit that uses an antenna of a $50 \Omega$ input impedance. The maximum conversion efficiency of the rectenna system that uses our proposed design was approximately $64 \%$ at $2 \mathrm{GHz}$, whereas the maximum efficiency of the rectenna system with a matching circuit is approximately $70 \%$ at $2 \mathrm{GHz}$. These results prove that using the adjustable antenna and without the need for a matching circuit, I can obtain an efficiency that is very close to the efficiency of a rectenna system that uses an additional matching circuit. 


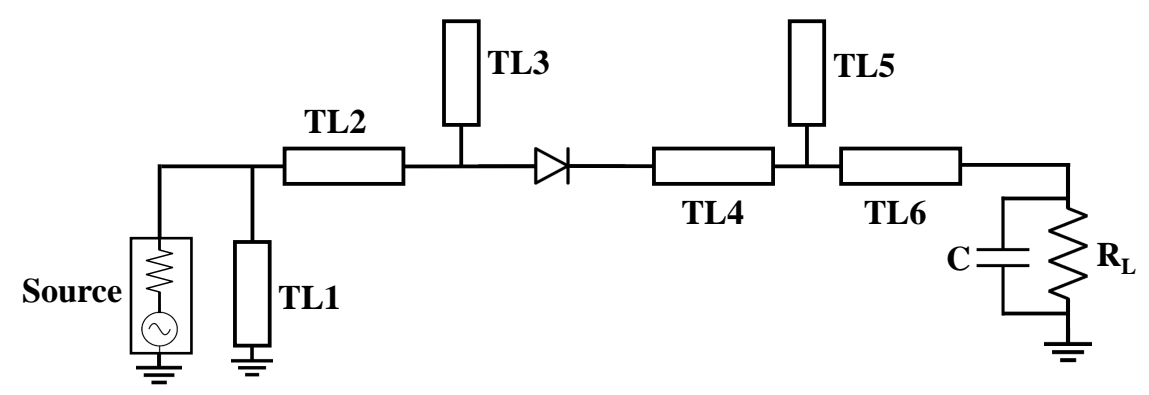

Figure 6.9: A schematic showing the rectifier circuit designed in ADS. The parameters are listed in Table 6.6. There is a total of 6 microstrip transmission line segments where TLn refers to the nth segment. The circuit is terminated by a parallel combination of load resistor of $4.5 \mathrm{~K} \Omega$ and a capacitor of $40 \mathrm{pF}$.

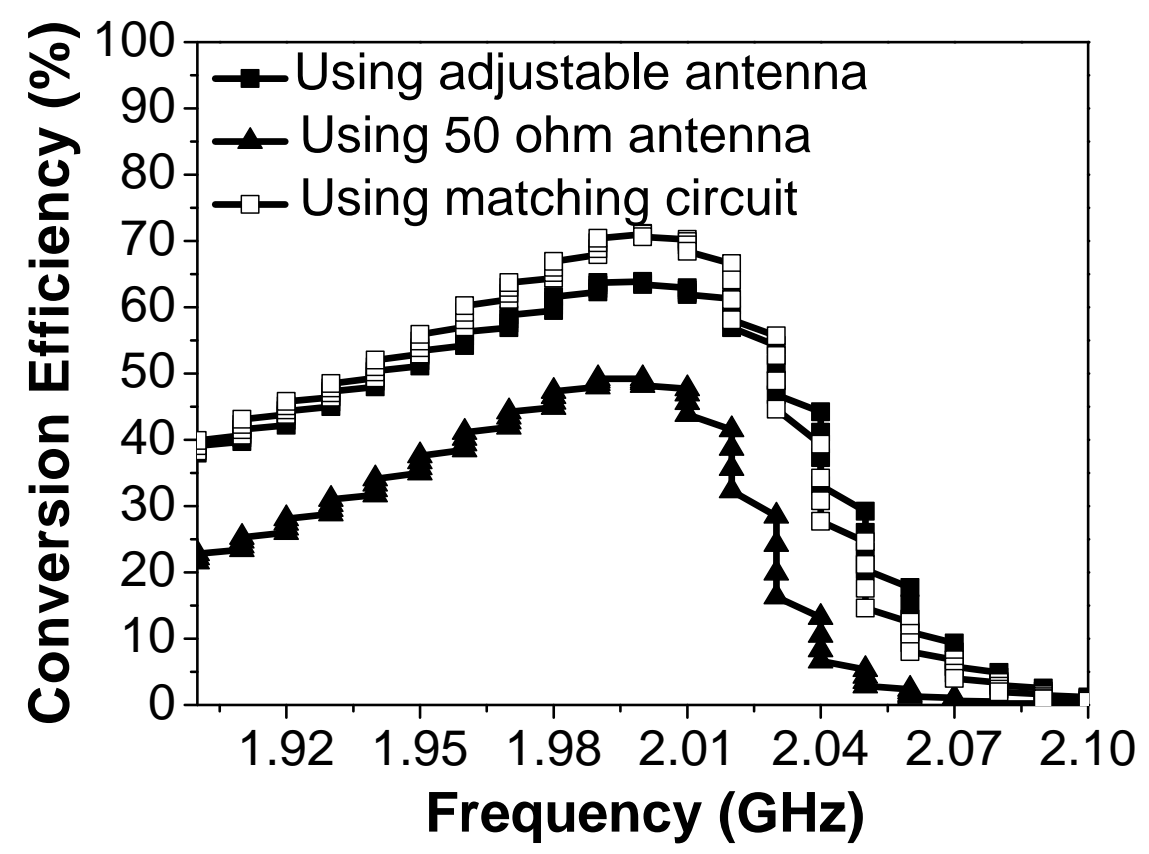

Figure 6.10: Conversion efficiencies; using adjustable antenna without a matching circuit, using $50 \Omega$ antenna without a matching circuit and using $50 \Omega$ antenna with a matching circuit. 


\subsection{Conclusion}

In this work, I have introduced a novel and simple controllable input impedance dipole antenna. Simulation results show the capability of controlling the input impedance of the proposed design. Both inductive and capacitive input impedance can be obtained utilizing this design. Additionally, the effect of varying the dimensions of the design was investigated. A rectenna system utilizing the proposed dipole was designed and studied. Comparison between rectenna systems that use our proposed design without and with matching circuits was presented. The comparison results show that the rectenna that uses our design, without using a matching circuit, can achieve a conversion efficiency that is very close to that of the rectenna system with a matching circuit. Adding a matching circuit complicates the circuit and increases the losses in the circuit. Consequently, utilizing the proposed design I can overcome the problem of undesired complexity and losses. 


\section{Chapter 7}

\section{Accomplished and Future Work}

\subsection{Accomplished Work}

The work achieved in this thesis is summarized in the following list:

1. The ability of utilizing X-parameters to characterize a diode and a complete rectenna system was validated in Chapter 3. In this chapter, I introduced the concept of using the X-Parameter model to simulate nonlinear devices. Measurements or simulation can be used to extract the X-Parameters. The procedure for obtaining X-parameters with ADS and using them in simulation is detailed. The outcomes of the circuit model of a diode are compared to the results of the X-Parameters method. The comparison results demonstrate the applicability of X-Parameters and their ability to replicate nonlinear device behaviour.

To simulate a full rectenna circuit utilizing X-Parameters, the circuit was divided into two parts: electromagnetic and circuit. The source, transmitting antenna, and receiving antenna comprise the electromagnetic portion. An equivalent circuit, consisting of a power source and impedance, was used to replace the electromagnetic portion. The circuit, on the other hand, is made up of a matching circuit, a diode, and a load. The circuit part's X-Parameters were retrieved and used in simulation. A comparison was made between the X-Parameters model and the circuit model. The good agreement between the two methods demonstrates that X-Parameters can be used to replace circuit models that require a long time to construct.

2. A method to simulate, integrate and optimize a full rectenna circuit over a range of frequencies was presented and validated Chapter 4. I developed a way to allow 
full simulation or characterisation of a complete rectenna in this chapter. This is performed by substituting the receiving antenna with its Thevenin equivalent circuit, then combining the equivalent circuit with the rectification circuit and load using a circuit simulator.

Our method's main goal is to provide full rectenna simulation so that the designer may accurately estimate the rectenna's performance over the required frequency range and for any angle of incidence and polarisation. All of this may be performed without the use of fabrication or measurements. In fact, using our simulation method, one can create a detailed graphical chart that depicts a rectenna's performance under any excitation scenario (polarization, incidence angle, and power density). This capability enables the performance of any rectenna built by a third party to be evaluated without the need of measurements.

Another advantage of our method is its ability to optimize the rectification circuit for a specific receiving antenna to deliver maximum DC power. This advantage is critical since an optimal rectification circuit is strongly dependent on the particular antenna employed. Furthermore, utilizing this method, I was able to optimize the rectenna at various sub-bands of the operating spectrum.

3. In chapter 5 , I designed an MSA that operates at $5.8 \mathrm{GHz}$ and employs a feeding network to channel all received signals into a single port. In addition, I designed an MPA array that works at the same frequency using the same material and dimensions. Both designs are studied in flat and bent states to compare their performance. During conformal operation, the MSA provides stable realised gain and efficiency when compared to the MPA array, according to the comparison results. Furthermore, when the $\mathrm{x}$-axis is bent, the resonant frequency of the MSA is marginally altered for all selected values, whereas the resonant frequency of the MPA array is significantly modified during the bend scenario.

Because all of the comparison results show that the MSA is more stable in the ISM band at various curvatures, a rectenna system based on the proposed MSA was created to operate at $5.8 \mathrm{GHz}$. The rectenna proposed has been tuned for excellent AC-to-DC conversion efficiency. At $5.8 \mathrm{GHz}$, an AC-to-DC conversion efficiency of around $81 \%$ was achieved.

4. In chapter 6, I have introduced a novel and simple controllable input impedance dipole antenna. Simulation results show the capability of controlling the input impedance of the proposed design. Both inductive and capacitive input impedance can be obtained utilizing this design. Additionally, the effect of varying the dimensions of the design 
was investigated. A rectenna system utilizing the proposed dipole was designed and studied. Comparison between rectenna systems that use our proposed design without and with matching circuits was presented. The comparison results show that the rectenna that uses our design, without using a matching circuit, can achieve a conversion efficiency that is very close to that of the rectenna system with a matching circuit. Adding a matching circuit complicates the circuit and increases the losses in the circuit. Consequently, utilizing the proposed design I can overcome the problem of undesired complexity and losses.

\subsection{List of Publications}

1. Melad M. Olaimat, and Omar M. Ramahi, Simulation and Optimization of Rectenna, (Under review with IEEE Transactions on Microwave Theory and Techniques).

2. Melad M. Olaimat, Leila Yousefi and Omar M., Using Plasmonics and Nanoparticles to Enhance the Efficiency of Solar Cells: Review of Latest Technologies, The Journal of the Optical Society of America, December 2020.

3. Melad M. Olaimat, Youcef Braham, Mohamed El Badawe, Mourad Nedil, and Omar M. Ramahi, Effect of Bending on Metasurface Antenna and Microstrip Patch Antenna Array, (Accepted in 2021 IEEE AP-S Symposium on Antennas and Propagation and USNC-URSI Radio Science).

4. Melad M. Olaimat, and Omar M. Ramahi, Simulation of Rectenna, (Accepted in 2021 IEEE AP-S Symposium on Antennas and Propagation and USNC-URSI Radio Science).

5. Melad M. Olaimat, and Omar M. Ramahi, Adjustable Input Impedance Dipole Antenna, (Accepted in 2021 IEEE 19th International Symposium on Antenna Technology and Applied Electromagnetics (ANTEM)).

6. Melad M. Olaimat, and Omar M. Ramahi, Simulation and Optimization of Rectenna, (Accepted in 2021 Indian Conference on Antennas and Propagation (InCAP 2021)).

7. Tao Tang, Melad M. Olaimat, Maged Aldhaeebi, Guangjun Wen, Li Xiao, Circular multi-usage RFID tag antenna with coding ability for chipless application, International Journal of RF and Microwave Computer-Aided Engineering, September 2020. 
8. Youcef Braham, Mourad Nedil, Melad M. Olaimat, Mohamed El Badawe, and Omar M. Ramahi, Wearable Metasurface Antenna based on Electrically-small Ring Resonators for WBAN Applications, Accepted in IET Microwaves, Antennas and Propagation, October 2021.

\section{3 $\quad$ Future Work}

1. Utilization of the method proposed in Chapter 4 to design efficient rectenna circuits that operate over wide-band of frequencies.

2. Extend the work in Chapter 5 to design rectenna circuits that operate at high frequency.

3. Develop a rectenna circuit that can efficiently absorb power from various angles since the proposed antenna in Chapter 5 is stable with bending.

4. Fabricate a prototype of a compact rectenna design where the antenna is directly matched to the diode using the design from Chapter 6. 


\section{References}

[1] Our world in data. https://ourworldindata.org/renewable-energy. Accessed: July 2021.

[2] Guneet Bedi and Rajendra Singh. Quantum dot solar cells. In Nanotechnology (IEEE-NANO), 2017 IEEE 17th International Conference on, pages 225-229. IEEE, 2017.

[3] Alessandra Costanzo, Marco Dionigi, Diego Masotti, Mauro Mongiardo, Giuseppina Monti, Luciano Tarricone, and Roberto Sorrentino. Electromagnetic energy harvesting and wireless power transmission: A unified approach. Proceedings of the IEEE, 102(11):1692-1711, 2014.

[4] Solar of solar power satellite system. https://www.google.com/search?q=solar+ power+satellite\&rlz=1C1CHBF_enCA855CA855\&sxsrf=ADaemvLoDoQUQt7z64atTX97nIHALVEeA: $1635153063210 \&$ source=lnms\&tbm=isch\&sa=X\&ved= 2ahUKEwj-h_Hrm-XzAhVMSjABHY2fASsQ_AUoAXoECAEQAw\&biw=1163\&bih=553\& dpr=2.2\#imgrc=1HE5_0xY1_t3LM. Accessed: October 2021.

[5] Garret Moddel. Will rectenna solar cells be practical? In Rectenna Solar Cells, pages 3-24. Springer, 2013.

[6] Ramesh Garg, Prakash Bhartia, Inder J Bahl, and Apisak Ittipiboon. Microstrip antenna design handbook. Artech house, 2001.

[7] Prince Mahdi Masud. A methodology for designing $2.45 \mathrm{ghz}$ wireless rectenna system utilizing dickson charge pump with optimized power efficiency. Master's thesis, University of Waterloo, 2013.

[8] Hewlett Packard. Technical data, “. Surface Mount Microwave Schottky Detector Diodes, 1999. 
[9] Vahid Khoshdel and Mehrdad Shokooh-Saremi. Design and optimization of slot nano-antennas for ambient thermal energy harvesting. Optik-International Journal for Light and Electron Optics, 138:470-475, 2017.

[10] Harry A Atwater and Albert Polman. Plasmonics for improved photovoltaic devices. Nature materials, 9(3):205, 2010.

[11] Efficiency of silicon solar cells climbs. https://spectrum.ieee.org/energywise/ energy/renewables/efficiency-of-solar-cells-continues-to-climb. Accessed: July 2021.

[12] Fraunhofer ise and evg set $31.3 \%$ efficiency for silicon multi-junction solar cell. https://www.pv-magazine.com/2017/03/24/fraunhofer-ise-and-evgset-31-3-efficiency-for-silicon-multi-junction-solar-cell/. Accessed: July 2021.

[13] Seung Yeop Myong. Current status of inorganic solar cells using quantum structures. Recent patents on nanotechnology, 6(1):2-9, 2012.

[14] Octavi E Semonin, Joseph M Luther, and Matthew C Beard. Quantum dots for next-generation photovoltaics. Materials today, 15(11):508-515, 2012.

[15] Robert Leo Bailey. A proposed new concept for a solar-energy converter. Journal of Engineering for Power, 94(2):73-77, 1972.

[16] Ahmed Z Ashoor, Thamer S Almoneef, and Omar M Ramahi. A planar dipole array surface for electromagnetic energy harvesting and wireless power transfer. IEEE Transactions on Microwave Theory and Techniques, 66(3):1553-1560, 2017.

[17] Thamer S Almoneef, Faruk Erkmen, and Omar M Ramahi. Harvesting the energy of multi-polarized electromagnetic waves. Scientific reports, 7(1):14656, 2017.

[18] Alexandru Takacs, Abderrahim Okba, Hervé Aubert, Samuel Charlot, and PierreFrançois Calmon. Recent advances in electromagnetic energy harvesting and wireless power transfer for iot and shm applications. In 2017 IEEE International Workshop of Electronics, Control, Measurement, Signals and their Application to Mechatronics (ECMSM), pages 1-4. IEEE, 2017.

[19] James C Maxwell. A dynamical theory of the electromagnetic field. Wipf and Stock Publishers, 1996. 
[20] Margaret Cheney. Tesla: man out of time. Simon and Schuster, 2011.

[21] JF Skowron, GH MacMaster, and WC Brown. The super power cw amplitron. Microwave J., 7, 1964.

[22] Peter E Glaser. An overview of the solar power satellite option. IEEE Transactions on Microwave Theory and Techniques, 40(6):1230-1238, 1992.

[23] Susumu Sasaki, Koji Tanaka, and Ken-ichiro Maki. Microwave power transmission technologies for solar power satellites. Proceedings of the IEEE, 101(6):1438-1447, 2013.

[24] JJ Schlesak, A Alden, and T Ohno. Sharp (stationary high altitude relay platform)rectenna and low altitude tests. In GLOBECOM'85-Global Telecommunications Conference, volume 2, pages 960-964, 1985.

[25] H Matsumoto, N Kaya, I Kimura, S Miyatake, M Nagatomo, and T Obayashi. Minix project toward the solar power satellite-rocket experiment of microwave energy transmission and associated nonlinear plasma physics in the ionosphere. In Proc. ISAS Space Energy Symp., pages 69-76, 1982.

[26] N Kaya, H Matsumoto, and R Akiba. Rocket experiment mets-microwave energy transmission in space. SPS 91-Power from Space, pages 336-342, 1991.

[27] Ari Juels. Rfid security and privacy: A research survey. IEEE journal on selected areas in communications, 24(2):381-394, 2006.

[28] Fu-Jhuan Huang, Chien-Ming Lee, Chia-Lin Chang, Liang-Kai Chen, Tzong-Chee Yo, and Ching-Hsing Luo. Rectenna application of miniaturized implantable antenna design for triple-band biotelemetry communication. IEEE Transactions on Antennas and Propagation, 59(7):2646-2653, 2011.

[29] Liguang Xie, Yi Shi, Y Thomas Hou, and Andwenjing Lou. Wireless power transfer and applications to sensor networks. IEEE Wireless Communications, 20(4):140-145, 2013.

[30] Joseph Polastre, Jason Hill, and David Culler. Versatile low power media access for wireless sensor networks. In Proceedings of the 2nd international conference on Embedded networked sensor systems, pages 95-107, 2004.

[31] Ian F Akyildiz, Weilian Su, Yogesh Sankarasubramaniam, and Erdal Cayirci. Wireless sensor networks: a survey. Computer networks, 38(4):393-422, 2002. 
[32] Dionisis Kandris, Christos Nakas, Dimitrios Vomvas, and Grigorios Koulouras. Applications of wireless sensor networks: an up-to-date survey. Applied System Innovation, 3(1):14, 2020 .

[33] Pei-Cheng Hii and Wan-Young Chung. A comprehensive ubiquitous healthcare solution on an android ${ }^{\top M}$ mobile device. Sensors, 11(7):6799-6815, 2011.

[34] Priyanka Kakria, NK Tripathi, and Peerapong Kitipawang. A real-time health monitoring system for remote cardiac patients using smartphone and wearable sensors. International journal of telemedicine and applications, 2015, 2015.

[35] JeongGil Ko, Tia Gao, and Andreas Terzis. Empirical study of a medical sensor application in an urban emergency department. In Proceedings of the fourth international conference on body area networks, pages 1-8, 2009.

[36] Adnan Nasir, Boon-Hee Soong, and Selvakumaran Ramachandran. Framework of wsn based human centric cyber physical in-pipe water monitoring system. In 2010 11th International Conference on Control Automation Robotics 8 Vision, pages 1257-1261. IEEE, 2010.

[37] Jaime Lloret, Sandra Sendra, Miguel Garcia, and Ginés Lloret. Group-based underwater wireless sensor network for marine fish farms. In 2011 IEEE GLOBECOM Workshops (GC Wkshps), pages 115-119. IEEE, 2011.

[38] Carlos Henrique Petzl Lorenz, Simon Hemour, and Ke Wu. Physical mechanism and theoretical foundation of ambient rf power harvesting using zero-bias diodes. IEEE Transactions on Microwave Theory and Techniques, 64(7):2146-2158, 2016.

[39] Zied Harouni, Laurent Cirio, Lotfi Osman, Ali Gharsallah, and Odile Picon. A dual circularly polarized 2.45 -ghz rectenna for wireless power transmission. IEEE Antennas and Wireless Propagation Letters, 10:306-309, 2011.

[40] Jui-Hung Chou, Ding-Bing Lin, Kuo-Lin Weng, and Hsueh-Jyh Li. All polarization receiving rectenna with harmonic rejection property for wireless power transmission. IEEE Transactions on Antennas and Propagation, 62(10):5242-5249, 2014.

[41] Mahima Arrawatia, Maryam Shojaei Baghini, and Girish Kumar. Broadband bent triangular omnidirectional antenna for rf energy harvesting. IEEE Antennas and Wireless Propagation Letters, 15:36-39, 2015. 
[42] C Bahhar, M Aidi, F Mejri, and T Aguili. Design and optimization of high-efficiency rectenna for rf energy harvesting. In 2019 PhotonIcs $\mathcal{E} 3$ Electromagnetics Research Symposium-Spring (PIERS-Spring), pages 2098-2105. IEEE, 2019.

[43] Huan Mei, Xuexia Yang, Bing Han, and Guannan Tan. High-efficiency microstrip rectenna for microwave power transmission at ka band with low cost. IET Microwaves, Antennas 83 Propagation, 10(15):1648-1655, 2016.

[44] Chaoyun Song, Yi Huang, Jiafeng Zhou, Paul Carter, Sheng Yuan, Qian Xu, and Zhouxiang Fei. Matching network elimination in broadband rectennas for highefficiency wireless power transfer and energy harvesting. IEEE Transactions on Industrial Electronics, 64(5):3950-3961, 2016.

[45] Ali Mavaddat, Seyyed Hossein Mohseni Armaki, and Ali Reza Erfanian. Millimeterwave energy harvesting using $4 \times 4$ microstrip patch antenna array. IEEE Antennas and wireless propagation letters, 14:515-518, 2014.

[46] Mohammod Ali, Guangli Yang, and Roger Dougal. Miniature circularly polarized rectenna with reduced out-of-band harmonics. IEEE antennas and wireless propagation letters, 5:107-110, 2006.

[47] Nuno Borges Carvalho, Apostolos Georgiadis, Alessandra Costanzo, Hendrik Rogier, Ana Collado, José Angel García, Stepan Lucyszyn, Paolo Mezzanotte, Jan Kracek, Diego Masotti, et al. Wireless power transmission: R\&d activities within europe. IEEE Transactions on Microwave Theory and Techniques, 62(4):1031-1045, 2014.

[48] Yen-Sheng Chen and Cheng-Wei Chiu. Maximum achievable power conversion efficiency obtained through an optimized rectenna structure for rf energy harvesting. IEEE Transactions on Antennas and Propagation, 65(5):2305-2317, 2017.

[49] Chaoyun Song, Yi Huang, Jiafeng Zhou, and Paul Carter. Improved ultrawideband rectennas using hybrid resistance compression technique. IEEE Transactions on Antennas and Propagation, 65(4):2057-2062, 2017.

[50] Chaoyun Song, Yi Huang, Paul Carter, Jiafeng Zhou, Sheng Yuan, Qian Xu, and Muayad Kod. A novel six-band dual cp rectenna using improved impedance matching technique for ambient rf energy harvesting. IEEE Transactions on Antennas and Propagation, 64(7):3160-3171, 2016. 
[51] Veronique Kuhn, Cyril Lahuec, Fabrice Seguin, and Christian Person. A multi-band stacked rf energy harvester with rf-to-dc efficiency up to $84 \%$. IEEE transactions on microwave theory and techniques, 63(5):1768-1778, 2015.

[52] Md Kamal Hosain, Abbas Z Kouzani, Mst Fateha Samad, and Susannah J Tye. A miniature energy harvesting rectenna for operating a head-mountable deep brain stimulation device. IEEE access, 3:223-234, 2015.

[53] Melad Olaimat and Aws Al-Qaisi. Novel designs of broadband patch antenna for wireless communication. International Journal of Computer Networks and Communications (IJCNC), 7(3):161-169, May 2015.

[54] Seok H Choi, Jong K Park, Sun K Kim, and Jae Y Park. A new ultra-wideband antenna for uwb applications. Microwave and optical technology letters, 40(5):399401, 2004.

[55] J Kim, CS Cho, and JW Lee. 5.2 ghz notched ultra-wideband antenna using slot-type srr. Electronics Letters, 42(6):315-316, 2006.

[56] Jeong Il Kim, Byung Moo Lee, and Young Joong Yoon. Wideband printed dipole antenna for multiple wireless services. In Proceedings RAWCON 2001. 2001 IEEE Radio and Wireless Conference (Cat. No. 01EX514), pages 153-156. IEEE, 2001.

[57] FJ Wang and JS Zhang. Wideband printed dipole antenna for multiple wireless services. Journal of Electromagnetic Waves and Applications, 21(11):1469-1477, 2007.

[58] Jouko Heikkinen and Markku Kivikoski. A novel dual-frequency circularly polarized rectenna. IEEE Antennas and Wireless Propagation Letters, 2:330-333, 2003.

[59] Joseph A Hagerty, Florian B Helmbrecht, William H McCalpin, Regan Zane, and Zoya B Popovic. Recycling ambient microwave energy with broad-band rectenna arrays. IEEE Transactions on Microwave Theory and Techniques, 52(3):1014-1024, 2004 .

[60] Yong Huang, Naoki Shinohara, and Hiroshi Toromura. A wideband rectenna for 2.4 ghz-band rf energy harvesting. In 2016 IEEE Wireless Power Transfer Conference (WPTC), pages 1-3. IEEE, 2016.

[61] Chaoyun Song, Yi Huang, Jiafeng Zhou, Jingwei Zhang, Sheng Yuan, and Paul Carter. A high-efficiency broadband rectenna for ambient wireless energy harvesting. IEEE Transactions on Antennas and Propagation, 63(8):3486-3495, 2015. 
[62] Wen-Hua Tu, Shih-Hsun Hsu, and Kai Chang. Compact 5.8-ghz rectenna using stepped-impedance dipole antenna. IEEE Antennas and Wireless Propagation Letters, 6:282-284, 2007.

[63] Ning Zhu, Richard W Ziolkowski, and Hao Xin. A metamaterial-inspired, electrically small rectenna for high-efficiency, low power harvesting and scavenging at the global positioning system 11 frequency. Applied Physics Letters, 99(11):114101, 2011.

[64] Shabnam Ladan, Ajay Babu Guntupalli, and Ke Wu. A high-efficiency 24 ghz rectenna development towards millimeter-wave energy harvesting and wireless power transmission. IEEE Transactions on Circuits and Systems I: Regular Papers, 61(12):3358-3366, 2014.

[65] Yu-Jiun Ren and Kai Chang. 5.8-ghz circularly polarized dual-diode rectenna and rectenna array for microwave power transmission. IEEE Transactions on Microwave Theory and Techniques, 54(4):1495-1502, 2006.

[66] Hucheng Sun, Yong-xin Guo, Miao He, and Zheng Zhong. A dual-band rectenna using broadband yagi antenna array for ambient rf power harvesting. IEEE Antennas and Wireless Propagation Letters, 12:918-921, 2013.

[67] Arslan Riaz, Subhan Zakir, Muhammad Musab Farooq, Muhammad Awais, and Wasif Tanveer Khan. A triband rectifier toward millimeter-wave frequencies for energy harvesting and wireless power-transfer applications. IEEE Microwave and Wireless Components Letters, 2020.

[68] Sandhya Chandravanshi, Kranti Kumar Katare, and MJ Akhtar. A flexible dualband rectenna with full azimuth coverage. IEEE Access, 9:27476-27484, 2021.

[69] Qiang Chen, Zhihao Liu, Yuguo Cui, Haotian Cai, and Xing Chen. A metallic waveguide-integrated 35-ghz rectenna with high conversion efficiency. IEEE Microwave and Wireless Components Letters, 30(8):821-824, 2020.

[70] JAG Akkermans, MC Van Beurden, GJN Doodeman, and HJ Visser. Analytical models for low-power rectenna design. IEEE Antennas and Wireless Propagation Letters, 4:187-190, 2005.

[71] Berndie Strassner and Kai Chang. 5.8-ghz circularly polarized dual-rhombic-loop traveling-wave rectifying antenna for low power-density wireless power transmission applications. IEEE Transactions on Microwave theory and Techniques, 51(5):15481553, 2003. 
[72] Faruk Erkmen, Thamer S Almoneef, and Omar M Ramahi. Electromagnetic energy harvesting using full-wave rectification. IEEE Transactions on Microwave Theory and Techniques, 65(5):1843-1851, 2017.

[73] Apostolos Georgiadis, G Vera Andia, and Ana Collado. Rectenna design and optimization using reciprocity theory and harmonic balance analysis for electromagnetic (em) energy harvesting. IEEE Antennas and Wireless Propagation Letters, 9:444446, 2010.

[74] James O McSpadden and John C Mankins. Space solar power programs and microwave wireless power transmission technology. IEEE microwave magazine, 3(4):46$57,2002$.

[75] Manuel Piñuela, Paul D Mitcheson, and Stepan Lucyszyn. Ambient rf energy harvesting in urban and semi-urban environments. IEEE Transactions on microwave theory and techniques, 61(7):2715-2726, 2013.

[76] M Nalini, JV Nirmal Kumar, R Muthu Kumar, and M Vignesh. Energy harvesting and management from ambient rf radiation. In 2017 International Conference on Innovations in Green Energy and Healthcare Technologies (IGEHT), pages 1-3. IEEE, 2017.

[77] Si Ce Wang, Min Jun Li, and Mei Song Tong. A high-performance rectenna for wireless power transfer in cubesats. IEEE Antennas and Wireless Propagation Letters, 19(12):2197-2200, 2020.

[78] Jialin Cai and Thomas J Brazil. X-parameter-based frequency doubler design. In 2012 7th European Microwave Integrated Circuit Conference, pages 794-797. IEEE, 2012.

[79] David E Root, Jan Verspecht, Jason Horn, and Mihai Marcu. X-Parameters: Characterization, Modeling, and Design of Nonlinear RF and Microwave Components. Cambridge University Press, 2013.

[80] Mohamed El Badawe, Thamer S Almoneef, and Omar M Ramahi. A metasurface for conversion of electromagnetic radiation to dc. AIP Advances, 7(3):035112, 2017.

[81] Mohammadreza F Imani, Jonah N Gollub, Okan Yurduseven, Aaron V Diebold, Michael Boyarsky, Thomas Fromenteze, Laura Pulido-Mancera, Timothy Sleasman, and David R Smith. Review of metasurface antennas for computational microwave imaging. IEEE Transactions on Antennas and Propagation, 68(3):1860-1875, 2020. 
[82] Mohamed El Badawe, Thamer S Almoneef, and Omar M Ramahi. A true metasurface antenna. Scientific reports, 6(1):1-8, 2016.

[83] Yahya SH Khraisat and Melad M Olaimat. Comparison between rectangular and triangular patch antennas array. In 2012 19th International Conference on Telecommunications (ICT), pages 1-5. IEEE, 2012.

[84] Ahmed Z Ashoor and Omar M Ramahi. Dielectric resonator antenna arrays for microwave energy harvesting and far-field wireless power transfer. Progress In Electromagnetics Research, 59:89-99, 2015.

[85] Constantine A Balanis. Antenna theory: analysis and design. John wiley \& sons, 2015.

[86] Georges A Deschamps. Microstrip microwave antennas. In Proceedings of the Third Symposium on the USAF Antenna Research and Development Program, Oct, pages 18-22, 1953.

[87] JQ Howell and Microstrip Antennas. Ieee ap-s int. In Symp. Digest, pages 177-180, 1972.

[88] R Munson. Conformal microstrip antennas and microstrip phased arrays. IEEE Transactions on Antennas and propagation, 22(1):74-78, 1974.

[89] Melad M Olaimat and Nihad I Dib. Improved formulae for the resonant frequencies of triangular microstrip patch antennas. International Journal of Electronics, 98(3):407-424, March 2011.

[90] Kin-Lu Wong. Compact and broadband microstrip antennas, volume 168. John Wiley \& Sons, 2004.

[91] Edward F Kuester, Mohamed A Mohamed, Melinda Piket-May, and Christopher L Holloway. Averaged transition conditions for electromagnetic fields at a metafilm. IEEE Transactions on Antennas and Propagation, 51(10):2641-2651, 2003.

[92] M Lapine and S Tretyakov. Contemporary notes on metamaterials. IET microwaves, antennas \&f propagation, 1(1):3-11, 2007.

[93] Karim Achouri, Mohamed A Salem, and Christophe Caloz. General metasurface synthesis based on susceptibility tensors. IEEE Transactions on Antennas and Propagation, 63(7):2977-2991, 2015. 
[94] Christopher L Holloway, Edward F Kuester, Joshua A Gordon, John O'Hara, Jim Booth, and David R Smith. An overview of the theory and applications of metasurfaces: The two-dimensional equivalents of metamaterials. IEEE Antennas and Propagation Magazine, 54(2):10-35, 2012.

[95] S Landy, N Iand Sajuyigbe, Jack J Mock, David R Smith, and Willie J Padilla. Perfect metamaterial absorber. Physical review letters, 100(20):207402, 2008.

[96] Xianliang Liu, Tatiana Starr, Anthony F Starr, and Willie J Padilla. Infrared spatial and frequency selective metamaterial with near-unity absorbance. Physical review letters, 104(20):207403, 2010.

[97] Thamer S Almoneef and Omar M Ramahi. Metamaterial electromagnetic energy harvester with near unity efficiency. Applied Physics Letters, 106(15):153902, 2015.

[98] David Shrekenhamer, Wangren Xu, Suresh Venkatesh, David Schurig, Sameer Sonkusale, and Willie J Padilla. Experimental realization of a metamaterial detector focal plane array. Physical review letters, 109(17):177401, 2012.

[99] Bernard Wathey, Jason Tierney, Pelle Lidström, and Jacob Westman. The impact of microwave-assisted organic chemistry on drug discovery. Drug discovery today, $7(6): 373-380,2002$.

[100] Joshua A Gordon, Christopher L Holloway, James Booth, Sung Kim, $\mathrm{Yu}$ Wang, James Baker-Jarvis, and David R Novotny. Fluid interactions with metafilms/metasurfaces for tuning, sensing, and microwave-assisted chemical processes. Physical Review B, 83(20):205130, 2011.

[101] Stephen A Maas. Nonlinear microwave and RF circuits. Artech house, 2003.

[102] Hakim Takhedmit. Modélisation et conception de circuits de réception complexes pour la Transmission d'Énergie Sans Fil à 2.45 GHz. PhD thesis, Ecully, Ecole centrale de Lyon, 2010.

[103] Agilent hsms-286x series surface mount microwave schottky detector diodes data sheet. https://datasheet.octopart.com/HSMS-2860-BLKG-Avago-datasheet7281230 .pdf. Accessed: August 2021.

[104] Advance design system. https://www.keysight.com/. Accessed: August 2021.

[105] Yuwei Zhou. Contribution to electromagnetic energy harvesting for wireless autonomousdevices. PhD thesis, UNIVERSITE DE NANTES, 2013. 
[106] agilent presentation. https://www.agilent.com/. Accessed: July 2021.

[107] David E Root, Jan Verspecht, David Sharrit, John Wood, and Alex Cognata. Broadband poly-harmonic distortion (phd) behavioral models from fast automated simulations and large-signal vectorial network measurements. IEEE Transactions on Microwave Theory and Techniques, 53(11):3656-3664, 2005.

[108] S Yahya, MM Olaimat, and SN Abdel-Razeq. Comparison between rectangular and triangular patch antenna arrays. Appl. Phys. Res, 4:1-5, 2012.

[109] Ping Lu, Xue-Song Yang, Jia-Lin Li, and Bing-Zhong Wang. A compact frequency reconfigurable rectenna for 5.2-and 5.8-ghz wireless power transmission. IEEE Transactions on Power Electronics, 30(11):6006-6010, 2014.

[110] Mei-Juan Nie, Xue-Xia Yang, Guan-Nan Tan, and Bing Han. A compact 2.45-ghz broadband rectenna using grounded coplanar waveguide. IEEE antennas and wireless propagation letters, 14:986-989, 2015.

[111] Xue-Xia Yang, Chao Jiang, Atef Z Elsherbeni, Fan Yang, and Ye-Qing Wang. A novel compact printed rectenna for data communication systems. IEEE transactions on antennas and propagation, 61(5):2532-2539, 2013.

[112] Kyrillos K Selim, Shaochuan Wu, and Demyana A Saleeb. Rf energy scavenging with a wide-range input power level. IEEE Access, 7:173450-173462, 2019.

[113] Paolo Ciampolini, Paolo Mezzanotte, Luca Roselli, and Roberto Sorrentino. Accurate and efficient circuit simulation with lumped-element fdtd technique. IEEE Transactions on microwave theory and techniques, 44(12):2207-2215, 1996.

[114] Computer system technology. https://www.3ds.com. Accessed: July 2021.

[115] V Rizzoli, D Masotti, N Arbizzani, and A Costanzo. Cad procedure for predicting the energy received by wireless scavenging systems in the near-and far-field regions. In 2010 IEEE MTT-S International Microwave Symposium, pages 1768-1771. IEEE, 2010 .

[116] Mohammad Alibakhshikenari, Bal S Virdee, Leyre Azpilicueta, Mohammad NaserMoghadasi, Mobayode Olusola Akinsolu, Chan Hwang See, Bo Liu, Raed A AbdAlhameed, Francisco Falcone, Isabelle Huynen, et al. A comprehensive survey of "metamaterial transmission-line based antennas: Design, challenges, and applications". IEEE Access, 8:144778-144808, 2020. 
[117] Victor Georgievich Veselago. The electrodynamics of substances with simultaneously negative values of $\epsilon$ and $\mu$. Physics-Uspekhi, 10(4):509-514, 1968.

[118] Omar M Ramahi, Thamer S Almoneef, Mohammed AlShareef, and Muhammed S Boybay. Metamaterial particles for electromagnetic energy harvesting. Applied Physics Letters, 101(17):173903, 2012.

[119] Miguel Ruphuy, Zhao Ren, and Omar M Ramahi. Flat far field lenses and reflectors. Progress In Electromagnetics Research, 34:163-170, 2014.

[120] David Schurig, Jack J Mock, BJ Justice, Steven A Cummer, John B Pendry, Anthony F Starr, and David R Smith. Metamaterial electromagnetic cloak at microwave frequencies. Science, 314(5801):977-980, 2006.

[121] Guo-Ping Gao, Bin Hu, Shao-Fei Wang, and Chen Yang. Wearable circular ring slot antenna with ebg structure for wireless body area network. IEEE Antennas and Wireless Propagation Letters, 17(3):434-437, 2018.

[122] Adel YI Ashyap, Samsul Haimi Bin Dahlan, Zuhairiah Zainal Abidin, Sharul Kamal Abdul Rahim, Huda A Majid, Abdulrahman SM Alqadami, and Mohamed El Atrash. Fully fabric high impedance surface-enabled antenna for wearable medical applications. IEEE Access, 9:6948-6960, 2021.

[123] W Brown, J Mims, and N Heenan. An experimental microwave-powered helicopter. In 1958 IRE International Convention Record, volume 13, pages 225-235. IEEE, 1966 .

[124] Constantine A Balanis. Antenna theory: analysis and design. John wiley \& sons, 2016.

[125] Melad Marzouq Olaimat and Nihad I Dib. A study of 15-75-90 angles triangular patch antenna. Progress In Electromagnetics Research, 21:1-9, February 2011.

[126] Hucheng Sun, Yong-xin Guo, Miao He, and Zheng Zhong. Design of a high-efficiency 2.45-ghz rectenna for low-input-power energy harvesting. IEEE Antennas and Wireless Propagation Letters, 11:929-932, 2012.

[127] Thamer S Almoneef, Hu Sun, and Omar M Ramahi. A 3-d folded dipole antenna array for far-field electromagnetic energy transfer. IEEE Antennas and Wireless Propagation Letters, 15:1406-1409, 2015. 
[128] James O McSpadden, Lu Fan, and Kai Chang. Design and experiments of a highconversion-efficiency 5.8-ghz rectenna. IEEE transactions on microwave theory and techniques, 46(12):2053-2060, 1998. 